\title{
Interplay of the COP9 signalosome deneddylase and the UspA deubiquitinase to coordinate fungal development and secondary metabolism
}

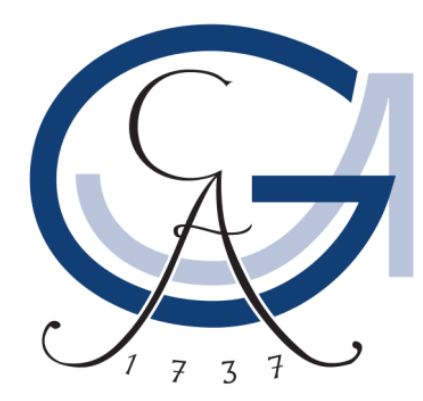

Dissertation

for the award of the degree

"Doctor rerum naturalium"

of the Georg-August-Universität Göttingen

within the doctoral program "Biomolecules: Stucture-Function-Dynamics" of the Georg-August University School of Science (GAUSS)

\author{
submitted by \\ Cindy Meister \\ from Schwarzhausen
}





\section{Thesis Committee:}

Referee: $\quad$ Prof. Dr. Gerhard H. Braus

Department of Molecular Microbiology and Genetics

Georg-August Universität Göttingen

$2^{\text {nd }}$ referee: Prof. Dr. Kai Tittmann

Department of Molecular Enzymology

Georg-August Universität Göttingen

$3^{\text {rd }}$ referee: Dr. Achim Dickmanns

Department of Structural Biology

Georg-August Universität Göttingen

Further members of the Examination Board:

Prof. Dr. Stefanie Pöggeler

Department of Genetics of Eukaryotic Microorganisms

Georg-August Universität Göttingen

Jun.-Prof. Dr. Kai Heimel

Department of Microbial Cell Biology

Georg-August Universität Göttingen

Prof. Dr. Heike Krebber

Department of Molecular Genetics

Georg-August Universität Göttingen

Date of oral examination: $6^{\text {th }}$ June 2018 



\section{Declaration of Independence}

Herewith I declare that the dissertation entitled "Interplay of the COP9 signalosome deneddylase and the UspA deubiquitinase to coordinate fungal development and secondary metabolism" was written on my own and independently without any other aids and sources than indicated.

Cindy Meister

Göttingen, 2018 

This work was conducted in the group of Prof. Dr. Gerhard H. Braus in the Department of Molecular Microbiology and Genetics, Institute of Microbiology and Genetics, Georg-AugustUniversität Göttingen.

Parts of this work are/will be published in:

Beckmann E.A., Köhler A.M., Meister C., Christmann M., Draht O.W., Rakebrandt N., Valerius O., Braus G.H., (2015). Integration of the catalytic subunit activates deneddylase activity in vivo as final step in fungal COP9 signalosome assembly. Mol. Microbiol. 97, 110-124.

Köhler A.M., Meister C., Braus G.H., (2015). In vitro deneddylation assay. Bio-protocol. 6, doi: 10.21769/BioProtoc. 1756

Meister C., Gulko M.K., Köhler AM., Braus G.H., (2016). The devil is in the details: comparison between COP9 signalosome (CSN) and the LID of the 265 proteasome. Curr. Genet. 62, 129136.

Meister C., Thieme S., Valerius O., Braus G.H., (2018). Interplay between COP9 signalosome deneddylase and the UspA/Usp15 deubiquitinase activities to control VeA velvet domain protein dependent fungal development. In preparation. 



\section{Table of Contents}

I Summary

II Zusammenfassung

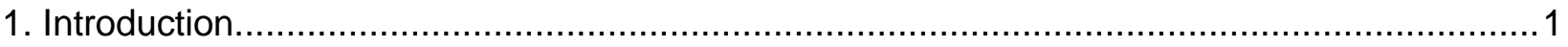

1.1 Posttranslational modifications regulate the fate of proteins ................................. 1

1.1.1 Ubiquitin - a powerful posttranslational modifier ............................................... 2

1.1.2 Ubiquitination of proteins by the concerted action of E1, E2 and E3 enzymes ............5

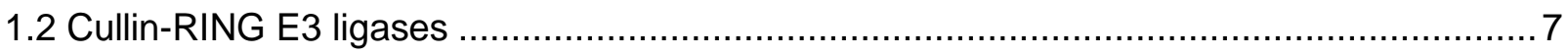

1.2.1 COP9 signalosome deneddylates E3 ubiquitin ligases ................................... 9

1.2.2 Interacting proteins of the COP9 signalosome .............................................. 11

1.3 Deubiquitinating enzymes reverse the ubiquitination process ................................... 12

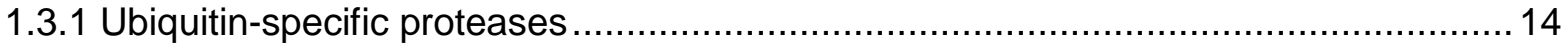

1.4 The genetic reference organism - Aspergillus nidulans ......................................... 15

1.4.1 Vegetative growth and multicellular reproduction of Aspergillus nidulans ................17

1.4.2 Molecular requirements for fungal multicellular development ...............................20

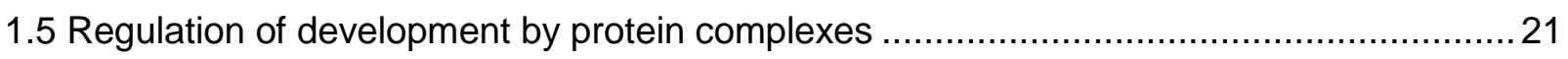

1.5.1 Velvet proteins - major regulators of fungal development ................................ 21

1.5.2 The COP9 signalosome in fungal development and secondary metabolism .............25

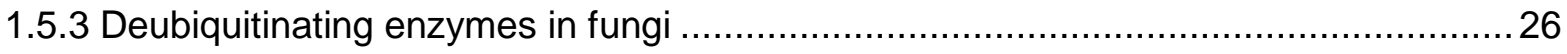

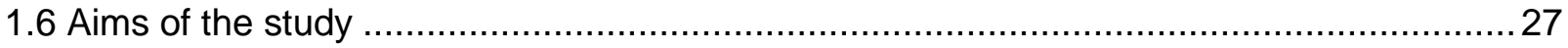

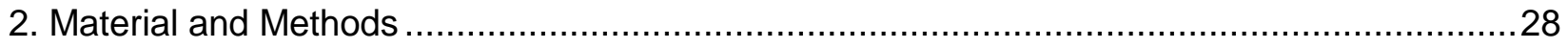

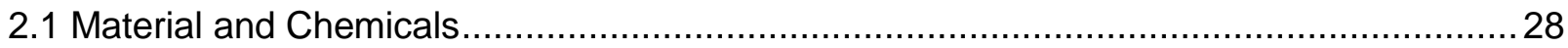

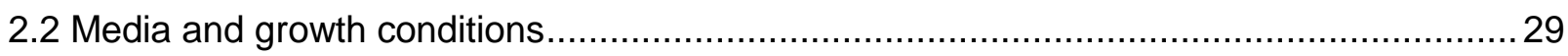

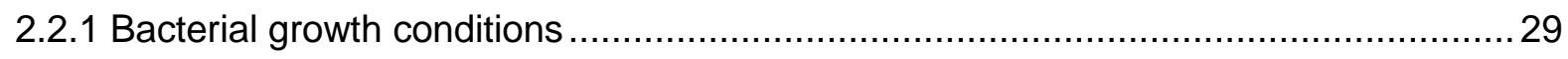

2.2.2 Aspergillus nidulans growth conditions ...................................................... 29

2.2.3 Saccharomyces cerevisiae growth conditions ................................................ 30

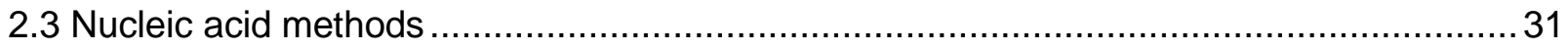

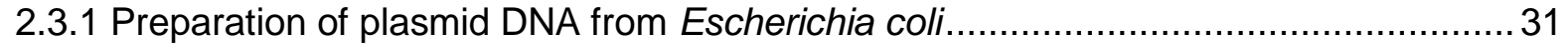

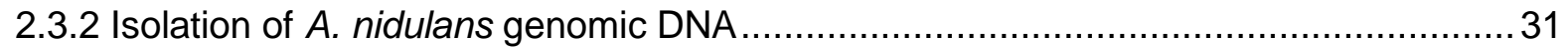

2.3.3 Polymerase chain reaction (PCR) ............................................................ 31

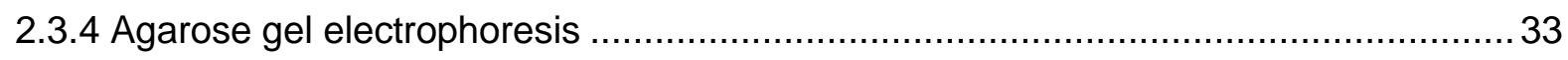

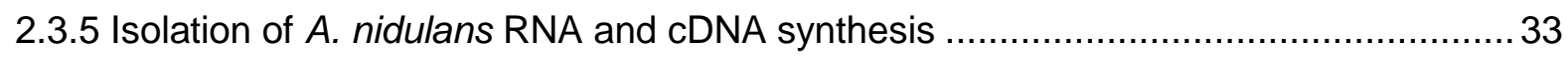


2.3.6 Quantitative real-time polymerase chain reaction ............................................ 34

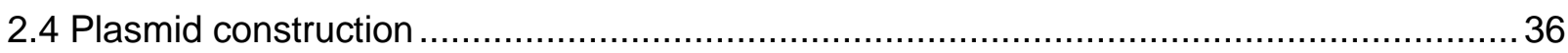

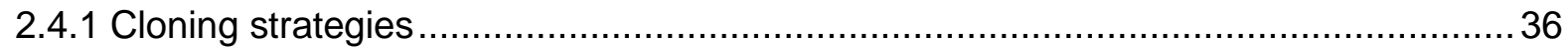

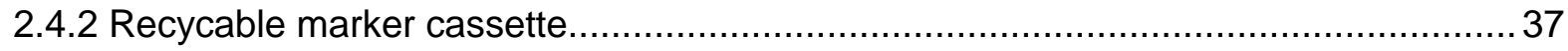

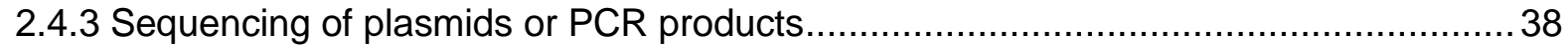

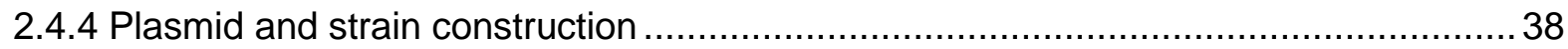

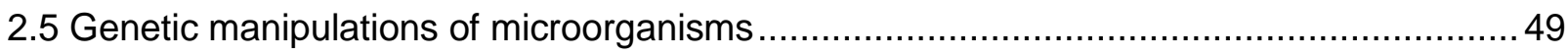

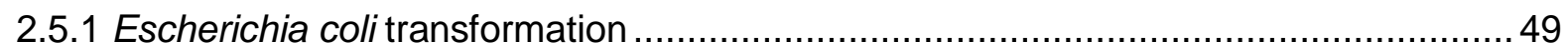

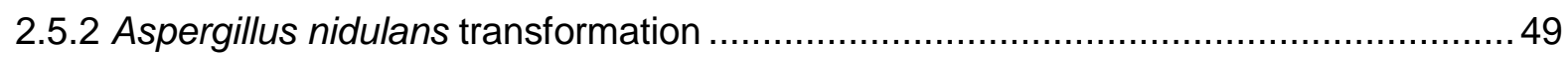

2.5.3 Saccharomyces cerevisiae transformation.................................................. 51

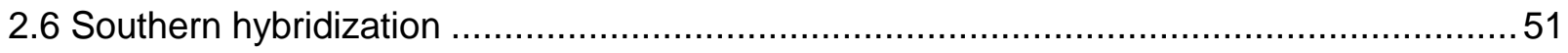

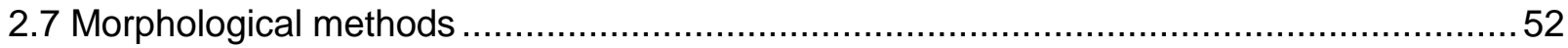

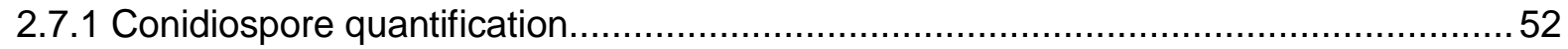

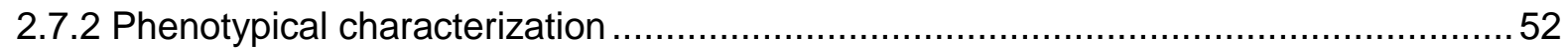

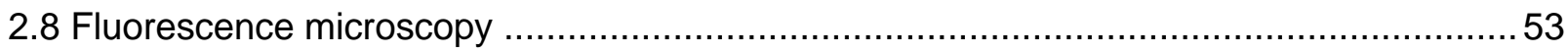

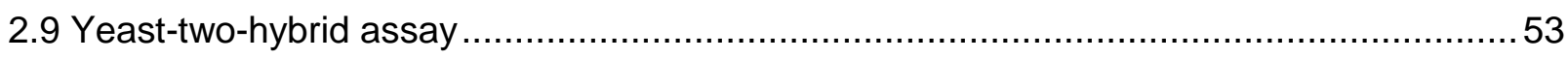

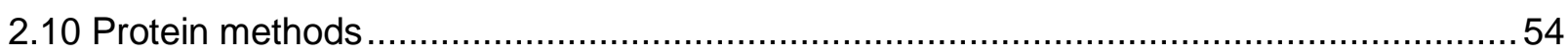

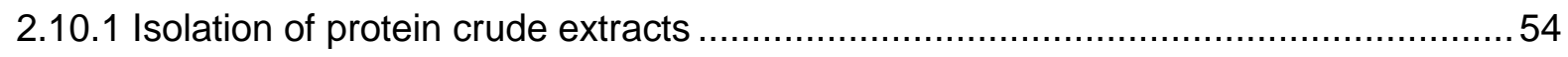

2.10.2 Sodium dodecyl sulfate polyacrylamide gel electrophoresis and western hybridization

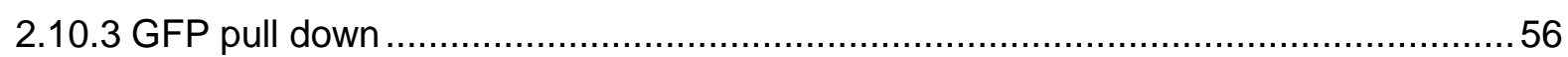

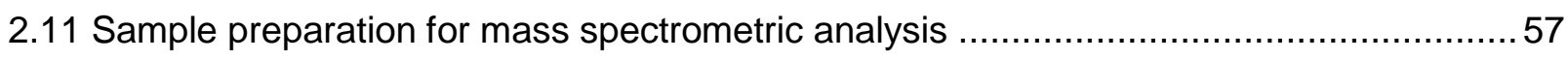

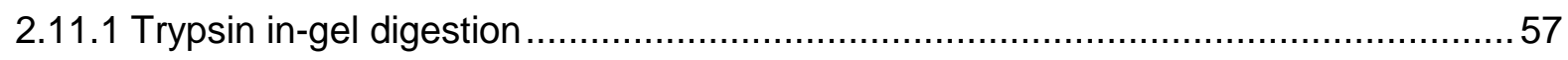

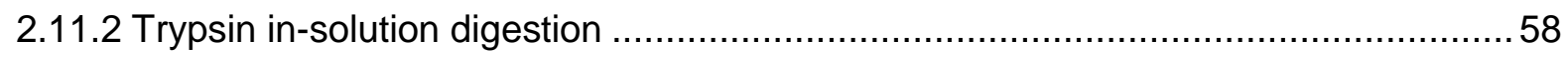

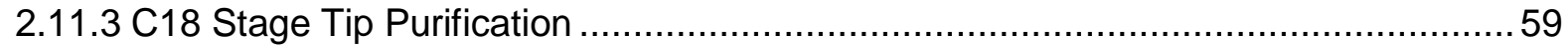

2.11.4 Liquid chromatography / mass spectrometry - mass spectrometry (LC/MS-MS) .....59

2.11.5 Data analysis with MaxQuant and Perseus................................................... 60

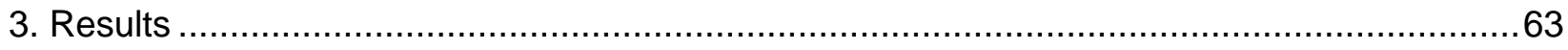

3.1 The COP9 signalosome mediates proteome changes during vegetative, filamentous

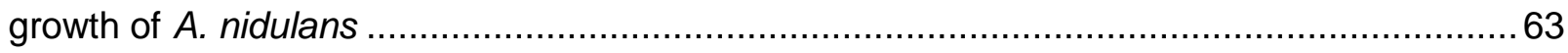

3.1.1 Establishing of a stable isotope labeling with amino acids (SILAC) protocol in A. nidulans

3.1.2 The COP9 signalosome changes more than $10 \%$ of the fungal proteome during vegetative growth. 
3.1.3 CsnE inhibits increased protein amounts of amino acid and vitamin metabolism, oxidoreductases and development related proteins during vegetative fungal growth ..........74

3.1.4 An intact COP9 signalosome promotes increased protein amounts of septins and cytoskeleton associated proteins during $A$. nidulans vegetative growth .......................... 77

3.2 A. nidulans ubiquitin-specific protease $\mathrm{A}$ interacts with the COP9 signalosome

3.2.1 The ubiquitin-specific protease A (UspA) encoding gene is located on chromosome I and encodes a 1,418 amino acid encompassing protein

3.2.2 A. nidulans UspA interacts with six subunits of the COP9 signalosome in a yeast-twohybrid assay 85

3.2.3 CsnE causes repressed transcript levels of the UspA encoding gene. 87

3.2.4 csnE transcript level and CsnE protein stability are independent of UspA.... 89

3.3 UspA is localized in proximity to and inside nuclei..... 91

3.3.1 UspA interacts with CsnB and CsnF in vivo in A. nidulans. 91

3.3.2 Active and inactive UspA is localized close to and within nuclei. .93

3.4 UspA activity requires C469 and C1066 to reduce the cellular pool of ubiquitinated proteins in the fungal cell.

3.5 UspA interacts with proteins involved in nuclear transport, RNA processing and the ubiquitin-proteasome system 99

3.6 UspA ensures coordinated fungal development and secondary metabolism ..... 105

3.6.1 UspA is required for asexual spore formation 105

3.6.2 UspA accelerates sexual development 109

3.6.3 UspA controls secondary metabolism.... 112

3.7 UspA alters protein levels of the major fungal regulator $\mathrm{VeA}$, but not of its interaction partner VelB during the initiation of multicellular development

3.7.1 VeA and VelB enter the nucleus and interact with each other independently of the deubiquitinase UspA.

4. Discussion

4.1 Regulation of protein abundance by the COP9 signalosome. 120

4.2 Aspergillus nidulans has a diverse repertoire of deubiquitinating enzymes

4.3 DUBs are often incorporated into multiprotein complexes during deubiquitination reactions 129

4.3.1 CsnE is required for regulation of the expression of DUB encoding genes 130

4.3.2 UspA interacts with subunits of the COP9 signalosome. 132

4.3.3 CsnE protein levels are independent of UspA. 133 
4.4 UspA reduces the cellular pool of ubiquitinated proteins during multicellular fungal development

4.4.1 UspA cleaves polyubiquitin chains that are bound to substrates

4.5 The localization and putative interaction partners of UspA suggest a role in controlling nuclear transport processes

4.6 UspA regulates the protein abundance of the major developmental regulator $\mathrm{VeA}$ and early multicellular development of $A$. nidulans. 140

4.6.1 $\mathrm{VeA}$ is a target for posttranslational modifications.

4.6.2 Velvet domain proteins have similarities to the mammalian NF-kB transcription factor family 144

4.7 Conclusion and outlook 147

5. References. 150

6. Supplementary Material 178

III List of Abbreviations $\mathrm{X}$

IV List of Tables XIV

$\mathrm{V}$ List of Figures $\mathrm{XV}$ 


\section{Summary}

Protein half-life is controlled through the ubiquitin-proteasome system (UPS). Proteins are labeled with ubiquitin chains by E3 cullin RING ligases (CRLs) and then degraded by the $26 \mathrm{~S}$ proteasome. The COP9 signalosome (CSN) is conserved between multicellular fungi and humans and inactivates CRLs by removing the ubiquitin-like protein Nedd8 from the cullin scaffold subunit (deneddylation). The conformational change of the E3 ligase complex allows the exchange of the substrate binding receptor complex. This enables ubiquitin-mediated degradation of different target proteins in response to environmental changes or developmental programs. CSN recognizes CRLs, which are not interacting with substrates. The proteomes of vegetative hyphae of $A$. nidulans with or without the gene encoding for the catalytically active deneddylase subunit CsnE of the CSN were compared. Therefore stable isotope labeling with amino acids in cell culture (SILAC) was established for this fungus. Relative quantification revealed changes in protein abundances of more than $10 \%$ of the identified proteins. An intact CSN promotes higher protein amounts of developmentally relevant proteins, whereas CSN dysfunction results in increased levels of proteins related to amino acid metabolism. The A. nidulans ubiquitin-specific protease $\underline{A}$ (UspA) counteracts the UPS by removing ubiquitin chains from proteins. UspA is the ortholog of human Usp15, which interacts with CSN. Gene expression of fungal $u s p A$ is reduced in the presence of a functional CSN. UspA interacts with fungal CSN subunits in vivo and in vitro and is primarily localized close to nuclei. The association of UspA to karyopherins and proteins involved in transcriptional processing imply a function during nuclear transport. The UspA cysteine residues C469 and C1066 are essential for its deubiquitination activity. Respective alanine exchanges in the usp $A^{A A}$ mutant allele produce an inactive enzyme resulting in increased levels of ubiquitinated proteins during fungal development similar to the $u s p A$ deletion strain. These results suggest a possible protein stabilization function of UspA. An uspA deletion strain forms reduced amounts of asexual conidia and is delayed in sexual fruiting body formation. Destabilization of $\mathrm{VeA}$ is observed during wild type development, but not without UspA. The VeA regulator of fungal development and secondary metabolism pulled only in uspA deficient strains the $\mathrm{DbaB}$ and $\mathrm{DbaH}$ proteins encoded by the derivative of benzaldehyde ( $d b a)$ secondary metabolite gene cluster. The lack of either functional UspA or CSN results in increased dba gene cluster expression. The location of UspA in proximity to the nucleus, the interaction with various proteins involved in nuclear transport, transcription and protein turnover and the impact on fungal development and secondary metabolism support a complex fine tuning function of the deubiquitinase in the $A$. nidulans life cycle. 


\section{Zusammenfassung}

Das Ubiquitin-Proteasom System reguliert die Lebensdauer der Proteine einer Zelle. Proteine,

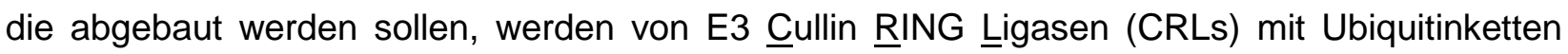
markiert und durch das 26S Proteasom abgebaut. Das COP9 Signalosom (CSN) ist von filamentösen Pilzen bis zum Menschen konserviert und deneddyliert CRLs. Dadurch kann ein Austausch der Rezeptor-Untereinheit stattfinden und es wird sichergestellt, dass je nach Umweltbedingungen oder Entwicklungsstadien spezifische Proteine zum Abbau markiert werden. Das COP9 Signalosom deneddyliert CRLs, die gerade kein Substrat binden. Im Rahmen dieser Arbeit wurde das Proteom vegetativer Hyphen in Abhängigkeit von der katalytisch aktiven CSN Untereinheit, CsnE, in Aspergillus nidulans analysiert. Dafür wurde SILAC (stable issotope labeling with amino acids in cell culture) für die Anwendung in dem filamentösen Pilz A. nidulans etabliert. Die relative Quantifizierung von Proteinen ergab, dass die Abundanz von $10 \%$ aller identifizierten Proteine in $\Delta c s n E$ im Vergleich zum Wildtyp verändert ist. Ein aktives COP9 Signalosom führt zu erhöhten Mengen von Proteinen, die für die pilzliche Entwicklung relevant sind, während es die Menge an Proteinen des Aminosäuremetabolismus reduziert. Die Ubiquitin-spezifische Protease $\underline{A}$ (UspA) in A. nidulans wirkt dem Ubiquitinierungsprozess entgegen und schneidet die Ubiquitinketten von den Substraten ab. UspA ist das Ortholog zum menschlichen Usp15. Die Genexpression von uspA ist reduziert, wenn das COP9 Signalosom funktioniert. UspA interagiert mit Untereinheiten des CSNs in vivo und in vitro und ist vorwiegend in der Nähe des Zellkerns lokalisiert. UspA könnte eine Funktion im Kerntransport ausüben, da es mit Karyopherinen sowie Proteinen, die an der Transkription beteiligt sind, interagiert. Die Menge an ubiquitinierten Proteinen wird während der gesamten pilzlichen Entwicklung von UspA reguliert. Dabei sind zwei Cysteine essentiell für die katalytische Aktivität. $\triangle u s p A$ bildet eine reduzierte Anzahl an Conidiosporen und ist verzögert in der Bildung sexueller Fruchtkörper. VeA, ein Regulator der pilzlichen Entwicklung und des sekundären Metabolismus (SM) wird im Wildtyp nach frühen Entwicklungszeitpunkten abgebaut, während es in $\triangle u s p A$ über die Zeit akkumuliert. VeA interagiert nur in $\triangle u s p A$ mit den Proteinen DbaB und $\mathrm{DbaH}$, welche durch das dba (derivative of benzaldehyde) SM Gen-Cluster codiert werden. Defekte im COP9 Signalosom oder in UspA führen zu einer erhöhten Expression der Gene dieses Clusters. Die Lokalisation von UspA in der Nähe des Zellkerns, die Interaktion mit Proteinen, die im Kerntransport, in der Transkription oder in der Proteinstabilität involviert sind sowie der Einfluss von UspA auf die Entwicklung und den SM von A. nidulans ermöglichen eine komplexe Steuerung des pilzlichen Lebenszyklus. 


\section{Introduction}

\subsection{Posttranslational modifications regulate the fate of proteins}

The three-dimensional fold of proteins is essential for their function. Tertiary structures of proteins are formed by different interactions of amino acid side groups, like disulfide or ionic bonds, Van-der-Waals bonds or hydrophobic interactions. Posttranslational modifications (PTMs) comprise the physical or chemical alteration of amino acids, which can modify the conformation, function, localization, stability or interaction network of proteins (Bah and Forman-Kay, 2016; Duan and Walther, 2015; Müller, 2017). This enables organisms to react on internal or external stimuli without changing the gene transcription process. Up to date, more than 200 different posttranslational modifications are known (Beltrao et al., 2013; Danielsen et al., 2011). The covalent attachment of small proteins (e.g. ubiquitin, small ubiquitin-like modifier (Sumo) or the neural precursor cell expressed, developmentally downregulated 8 (Nedd8)), carbohydrates (e.g. N- or O- linked glycosylation) or chemical groups (e.g. phospho-group, methyl-group, acetyl-group) at certain amino acids are reversible modifications, which alter the fate of the protein temporary (Prabakaran et al., 2012). Carbonylation is an irreversible modification, which makes the proteins dysfunctional and is associated to aging and different diseases (Karve and Cheema, 2011; Tanase et al., 2016). The most experimentally identified posttranslational modifications in eukaryotes comprise phosphorylations with more than 55,000 modified amino acids, followed by acetylations (more than 6,000) and $\mathrm{N}$-linked glycosylations (more than 5,000). PTMs can regulate a variety of processes including cell cycle, signaling cascades, DNA transcription or repair and many more, rendering the misregulation of PTMs a cause of many diseases (Ryan et al., 2014; Santos and Lindner, 2017).

Proteins can be modified with one or more PTMs at the same time, which results in PTM crosstalks (Hunter, 2007). Thereby, one PTM can serve as a trigger for the addition or removal of another one, which is known as positive cross-talk (Hunter, 2007; Nguyen et al., 2013; Prabakaran et al., 2012). Phosphorylation of proteins is often concomitant with subsequent ubiquitination events (Ciechanover et al., 2000; Ravid and Hochstrasser, 2008). For example, the inhibitors of the NF-KB transcription factor family (IKBs) become phosphorylated and therewith a substrate for the ubiquitin-proteasome system (UPS) (Oeckinghaus and Ghosh, 2009). Different PTMs can compete with each other for one amino acid in the protein, which is called negative PTM cross-talk. Lysine residues can be modified by several different PTMs, such as ubiquitination, sumoylation or acetylation, which cannot take place simultaneously (Danielsen 
et al., 2011; Hunter, 2007). Negative cross-talk between acetylation sites or di/tri-methylation sites was observed at histones (Schwämmle et al., 2016).

PTMs are widely distributed in the fungal kingdom and contribute to the regulation of fungal development, secondary metabolism and virulence (Leach and Brown, 2012). Phosphorylation changes the function of transcription factors and crucial master regulator proteins, such as the

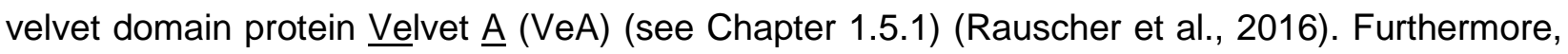
ubiquitin encoding genes are upregulated during stress conditions in Aspergillus nidulans, suggesting a role of ubiquitin as PTM in the general stress response of the fungus (Leach and Brown, 2012; Noventa-Jordão et al., 2000). It stays a major interest of current research to elucidate how PTMs contribute to fungal development or to the production of harmful or beneficial secondary metabolites.

\subsubsection{Ubiquitin - a powerful posttranslational modifier}

Ubiquitin is usually encoded by gene loci that represent head-to-tail fusions of many ubiquitin open reading frames (ORFs) or one ubiquitin ORF is fused to ribosomal protein encoding genes (Noventa-Jordão et al., 2000; Özkaynak et al., 1987; Wiborg et al., 1985). Humans contain a whole ubiquitin gene family (Wiborg et al., 1985). Fusion proteins containing up to nine ubiquitin head-to-tail repeats are described, but also genes encoding a single ubiquitin molecule are known (Wiborg et al., 1985). Ubiquitin is encoded as fusion protein of four head-to-tail repeats of ubiquitin by ubi4 or as fusion protein as $\mathrm{N}$-terminal extension of ribosomal protein called carboxyl extension protein (CEP) by ubi1 in $A$. nidulans (Noventa-Jordão et al., 2000). Four loci encode different ubiquitin fusions, namely ubi1 - ubi4, in Saccharomyces cerevisiae (Özkaynak et al., 1987). The translated ubiquitin fusion proteins are processed by deubiquitinating enzymes and result in single ubiquitin proteins that constitute the free cellular ubiquitin pool (Grou et al., 2015). Ubiquitin contains 76 amino acids and has a molecular weight of $8.5 \mathrm{kDa}$. The amino acid sequence is highly conserved among eukaryotic species, which indicates a conserved function (Figure 1). The characteristic di-glycine motif at its C-terminus is essential for the formation of isopeptide bonds between the $\epsilon-\mathrm{NH}_{2}$ group of lysine residues of target proteins and the ubiquitin molecule itself. Ubiquitin can be attached as single molecule to one amino acid residue of the target protein (monoubiquitination), to multiple amino acid residues of a protein (multiubiquitination) or can be attached as polyubiquitin chain at one residue of a protein (polyubiquitination) (Ohtake and Tsuchiya, 2017; Pickart and Eddins, 2004). The isopeptide bond in a polyubiquitin chain is formed between one of ubiquitin's seven lysine residues (K6, 
$\mathrm{K} 11, \mathrm{~K} 27, \mathrm{~K} 29$, K33, K48 and K63) or the initial methionine (M1) of one ubiquitin molecule and the di-glycine motif of the other molecule (Figure 1, 2; Spasser and Brik, 2012).

Modification of a protein with a single ubiquitin at a single lysine residue of the substrate has functions in endocytosis and DNA repair pathways (Hicke, 2001; Terrell et al., 1998; West and Bonner, 1980). Monoubiquitination of histones H2A or H2B influences transcription of genes in yeast and higher eukaryotes (Cao and Yan, 2012; Robzyk et al., 2000).

\section{H. sapiens \\ M.musculus \\ S. cerevisiae \\ A. nidulans}

H. sapiens

M.musculus

S. cerevisiae

A. nidulans
MQIFVKTLTGKTITLEVEPSDT IENVKAKIQDKEGIPP MQIFVKTLTGKTITLEVEPSDTIENVKAKIQDKEGIPP MQIFVKTLTGKTITLEVESSDTIDNVKSKIQDKEGIPP MQIFVKTLTGKTITLEVESSDTIDNVKTKIQDKEGIPP

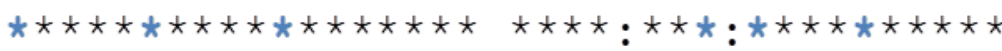

DQQRLIFAGKQLEDGRTLSDYNIQKESTLHLVLRLRGG DQQRLIFAGKQLEDGRTLSDYNIQKESTLHLVLRLRGG DQQRLIFAGKQLEDGRTLSDYNIQKESTLHLVLRLRGG DQQRLIFAGKQLEDGRTLSDYNIQKESTLHLVLRLRGG

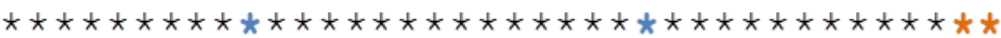

Figure 1: Ubiquitin is highly conserved between eukaryotes.

Multiple sequence alignments of single ubiquitin ORFs of Homo sapiens (Uniprot ID: P0CG48), Mus musculus (Uniprot ID: P0CG50), Saccharomyces cerevisiae (Uniprot ID: P0CG63) and Aspergillus nidulans (Uniprot ID: C8VLC7) were performed with the Clustal Omega alignment tool (Sievers et al., 2011). The seven lysine residues and the initial methionine are highlighted in blue. They can be used as a substrate for polyubiquitin chain formation. The C-terminal diglycine motif is required for isopeptide bond formation with a lysine residue of a substrate protein or another ubiquitin molecule and depicted here in orange. The only three non-conserved amino acid residues are highlighted in green. The last row of the alignment represents the conservation key (Chenna et al., 2003). The "“" indicates conserved amino acid residues among all the sequences used for this alignment. The ":" indicates a conservative mutation of the amino acid, meaning the exchange by another amino acid with similar chemical and physical properties. An empty space in the conservation key indicates non conserved residues.

The combinatorial possibilities generated by different ubiquitin linkages lead to a vast range of protein regulation through modification with polyubiquitin chains. Homotypic ubiquitin chains are always linked by only one lysine residue. The function of polyubiquitin chains linked through K6 or K27 remains unclear (Spasser and Brik, 2012). K11 linked chains were attributed to cell cycle signaling through tumor necrosis factor (TNF) and wingless-type (Wnt) signaling as well as to endoplasmatic reticulum-asssociated protein degradation (ERAD) (Bremm and Komander, 2011; 
Hay-Koren et al., 2011; Xu et al., 2009). K33 linked ubiquitin chains play a role in immune signaling, whereas K63 was linked to endocytosis, DNA damage response, selective autophagy and cell signaling cascades (Deng et al., 2000a; Lauwers et al., 2009; Spence et al., 1995; Tan et al., 2018; Yang et al., 2015). Linear ubiquitin chains linked through the initial methionine regulate NF-kB transcription factor family proteins (Ikeda, 2016; Rittinger and Ikeda, 2017). The complexity of ubiquitin modifications is multiplied by the fact that different lysine residues can be used for building up an ubiquitin chain. The function of these heterotypic or mixed ubiquitin chains remains still elusive. Another level of regulation is added by the posttranslational modifications of the ubiquitin chain itself due to phosphorylation, acetylation or sumoylation events (Ohtake and Tsuchiya, 2017; Sadowski et al., 2012; Spasser and Brik, 2012).

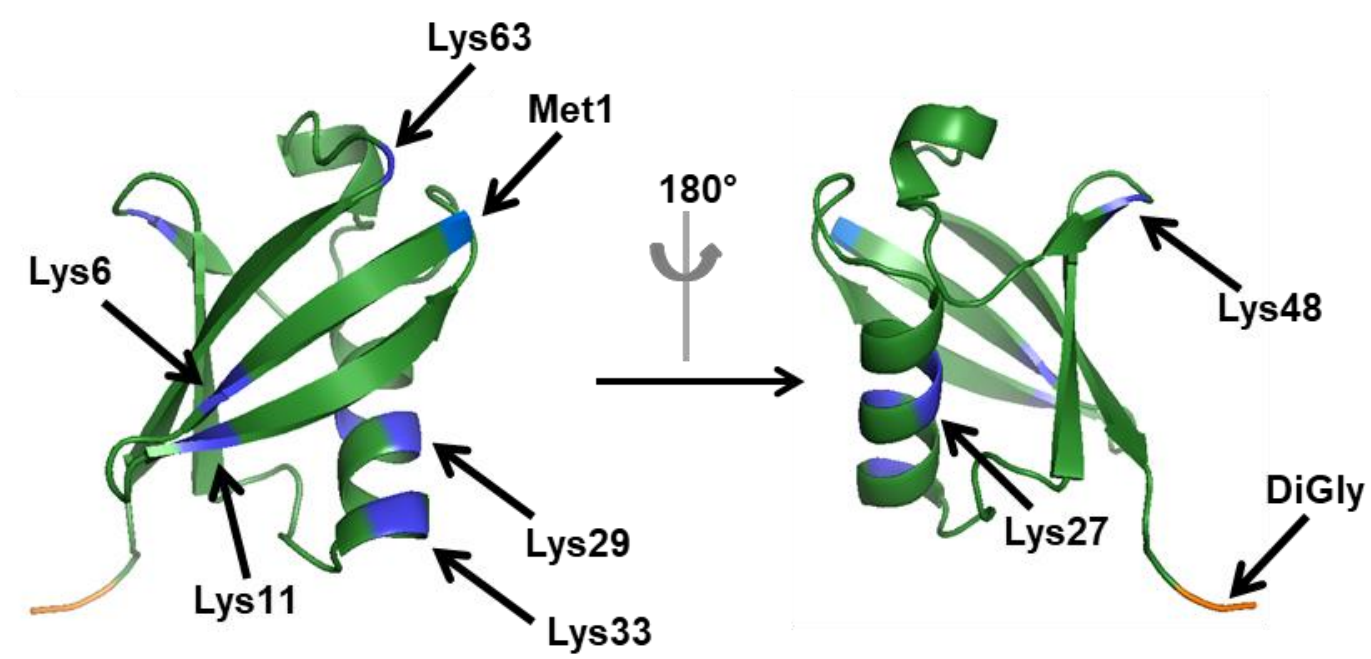

Figure 2: Localization of lysine residues in the three dimensional structure of ubiquitin. Cartoon representation of the crystal structure of the human ubiquitin molecule comprising 76 amino acids modified from PDB 1UBQ (Vijay-Kumar et al., 1987). The protruding di-glycine motif at the C-terminus is highlighted in orange. The seven conserved lysine residues (Lys) and the initial methionine (Met), which can be used for polyubiquitin chain formation, are depicted in blue. The crystal structure was modified using the PyMOL 2.0 software.

So far, the best-studied polyubiquitin chains are connected through K48 (Spasser and Brik, 2012; Xu et al., 2009). Substrates marked with an ubiquitin chain consisting of at least four ubiquitin molecules linked through K48 are mainly targeted to the 26S proteasome for degradation (Finley et al., 1994; Glickman and Ciechanover, 2002; Thrower et al., 2000). Proteasomal degradation is not restricted to K48 linked ubiquitin chains. Proteins modified with K11 were identified, which are substrates for proteasomal degradation as well (Xu et al., 2009). 
Linear ubiquitin chains linked through the initial methionine also induces the degradation by the 26S proteasome (Kirisako et al., 2006). Linear ubiquitin chains can bind to the $26 \mathrm{~S}$ proteasome regulatory particle and inhibit its degradative function (Saeki et al., 2004). A protein complex named linear ubiquitin chain assembly complex (LUBAC) investigated in human cell lines creates linear ubiquitin chains by the formation of an isopeptide bond between the C-terminal glycine of one and the amino group of the $\mathrm{N}$-terminal methionine of the other ubiquitin molecule (Kirisako et al., 2006). Ubiquitination in general is catalyzed by the orchestrated function of three enzymes: the E1 ubiquitin-activating, the E2 ubiquitin-conjugating and the E3 ubiquitin-ligating enzymes (Deshaies and Joazeiro, 2009; Finley et al., 2012).

\subsubsection{Ubiquitination of proteins by the concerted action of E1, E2 and E3 enzymes}

A quality control system for defective or misfolded proteins as well as protein half-life control in general is essential for the timely coordinated order of events inside a cell. The UPS marks proteins with ubiquitin and sentences them for degradation by the $26 \mathrm{~S}$ proteasome (Nandi et al., 2006; Wang and Maldonado, 2006). Different degradation signals, "degrons", label proteins for recognition by E3 ubiquitin ligases. Misfolded proteins often display larger amount of hydrophobic amino acids at their surface than correctly folded proteins. These hydrophobic degrons serve as recognition region for subsequent polyubiquitination reactions and are hidden in correctly folded proteins (Ravid and Hochstrasser, 2008). Protein half-life can depend on the $\mathrm{N}$-terminal residue(s), which control protein stability by the so-called "N-end rule pathway". The alteration of the $\mathrm{N}$-terminus by deamidation, acetylation or by processing of proteins with certain endopeptidases affects protein stability (Ravid and Hochstrasser, 2008; Tasaki et al., 2012). Phosphorylation of proteins often marks them for recognition by E3 ubiquitin ligases. These socalled phosphodegrons are mainly recognized by the Skp1-ㅡul1-ㅌbox (SCF) cullin ring ligase $(\mathrm{CRL})$ complexes, which are the largest family of multiprotein ubiquitin ligases (Ravid and Hochstrasser, 2008).

Ubiquitin is posttranslationally attached to proteins by the concerted action of E1 (ubiquitinactivating), E2 (ubiquitin-conjugating) and E3 (ubiquitin-ligating) enzymes (Deshaies and Joazeiro, 2009; Finley et al., 2012). E1 activates ubiquitin molecules in an ATP-dependent reaction by forming a thioester bond between its catalytic cysteine residue and the di-glycine motif at the C-terminus of ubiquitin (Figure 3). The activated ubiquitin is transferred to an E2 enzyme by formation of another thioester bond (Ye and Rape, 2011). Some E2 enzymes are specialized to initiate ubiquitin chain formation or to catalyze monoubiquitination by transferring 
ubiquitin molecules to a lysine residue of a certain substrate. Other E2 enzymes catalyze ubiquitin chain formation by binding and transferring ubiquitin preferentially to other ubiquitin molecules that are already bound to a substrate. These E2 enzymes bind the initial ubiquitin in a certain orientation and often confer linkage specificity (Ye and Rape, 2011).

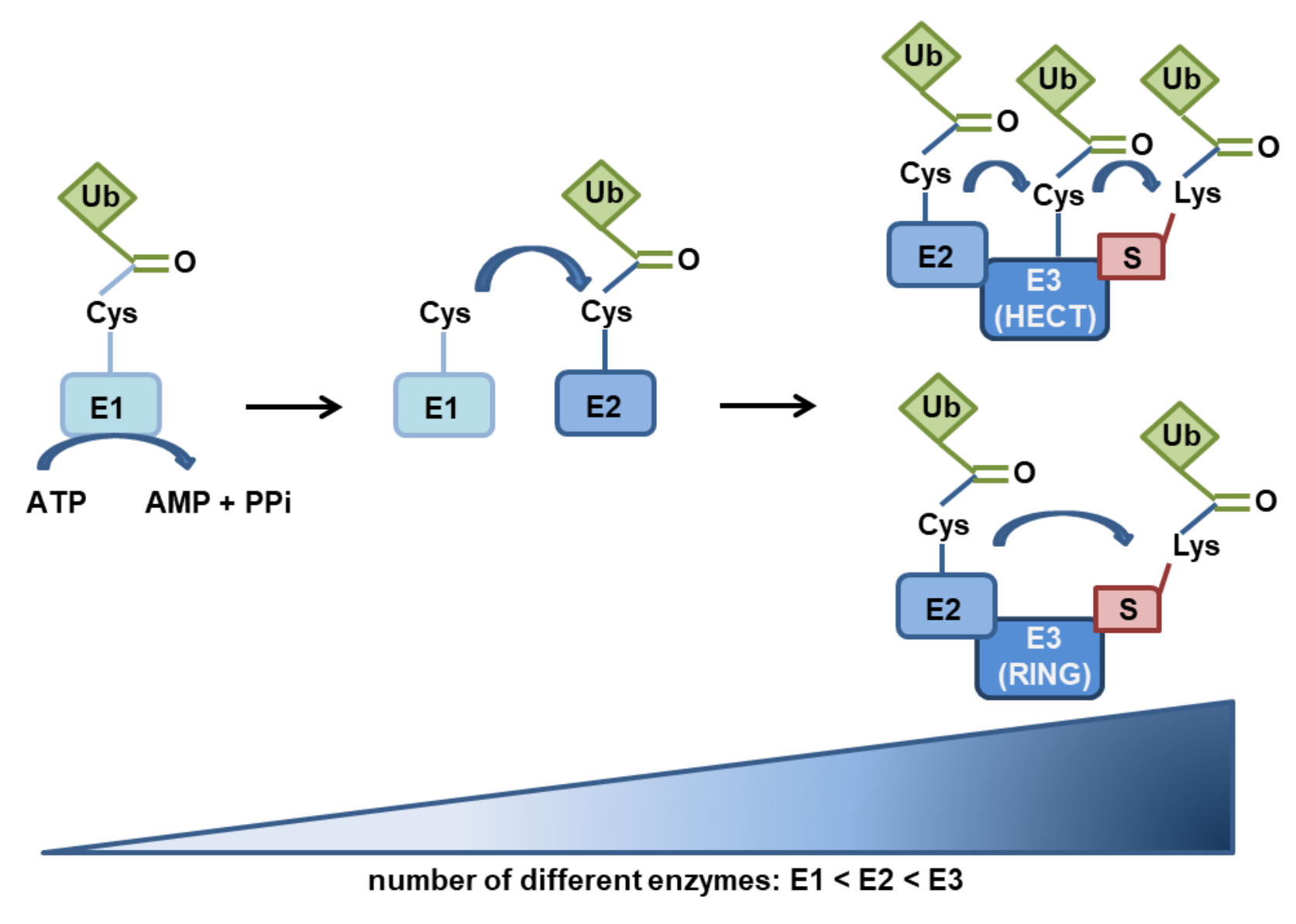

Figure 3: Transfer of ubiquitin molecules to target proteins.

Ubiquitin ( $\mathrm{Ub}$ ) becomes activated in an ATP-dependent reaction catalyzed by the E1 ubiquitinactivating enzyme. A highly energetic thioester bond is built between the cysteine residue of the E1 enzyme and the di-glycine motif at the C-terminal end of ubiquitin. Activated ubiquitin is transferred to an E2 enzyme. Some E2 enzymes are specialized for transferring the ubiquitin molecule directly on target substrates and initiate the ubiquitination processes. Other E2 enzymes rather function in chain elongation steps and transfer ubiquitin molecules to ubiquitins that are already attached to the substrate. E2 ubiquitin-conjugating enzymes interact with E3 ubiquitin ligases. There are two major classes of E3 enzymes: HECT ligases bind to the ubiquitin molecule prior to the transfer to the target substrate and RING domain E3 enzymes facilitate the transfer of the ubiquitin molecule from the E2 enzyme to the target substrate without binding to the ubiquitin molecule. 
The E2 ubiquitin-conjugating enzymes interact with the C-terminus of E3 ubiquitin ligases, which bring the E2 enzymes conjugated to ubiquitin and the substrate for ubiquitination into close proximity (Lecker et al., 2006).

Two major E3 ubiquitin ligase families exist: homologous to $\underline{E} 6$ associated protein $\underline{\mathrm{C}}$-terminus (HECT) and really interesting new gene (RING) E3 ligases (Figure 3). A direct transfer of ubiquitin from E2 to the substrate is catalyzed by RING E3 ligases. HECT domain E3 ligases take the ubiquitin from E2 to form a thioester-intermediate with its internal active site cysteine residue before the molecule is transferred to the target substrate (Glickman and Ciechanover, 2002).

The N-terminal sequence is highly variable in members of the HECT E3 ligase family, because this region is directly involved in substrate binding. RING domain E3 ligases can function as monomers or as multisubunit complexes. The multisubunit complexes encompass the binding to adaptor/receptor complexes, which ensure accurate substrate binding (Hershko and Ciechanover, 1998; Li et al., 2008; Metzger et al., 2010). The enzymes in the UPS are conserved in plants, excavates, chromalveolates and metazoans, including fungi and humans. Thereby, the number of E1, E2 and E3 enzymes increase exponentially (Hutchins et al., 2013; Ye and Rape, 2011). Only one E1 enzyme is characterized in S. cerevisiae, whereas in silico analyses predict three E1 enzymes (Hutchins et al., 2013). Furthermore, 11-13 E2 and 61 different E3 ubiquitin ligases are annotated in baker's yeast (Hutchins et al., 2013; Ye and Rape, 2011). Arabidopsis thaliana has two E1 enzymes, 37 E2 and more than 1,000 different E3 ubiquitin ligases (Hatfield et al., 1997; Kraft et al., 2005; Mazzucotelli et al., 2006).

Mammalian genomes encode approximately 30 HECT domain and circa 600 RING domain E3 ubiquitin ligases ( $\mathrm{Li}$ et al., 2008; Metzger et al., 2010). This reflects the level of complexity the single enzymes in the ubiquitination cascade have to deal with.

\subsection{Cullin-RING E3 ligases}

The largest family of E3 ligases are cullin RING E3 ligases (CRLs) (Petroski and Deshaies, 2005). They have a modular structure and consist of a Cullin1 protein, which serves as a scaffold. A $\underline{R} I N G$ box domain (Rbx) containing protein binds at cullins $\mathrm{C}$-terminus and a substrate adaptor/receptor complex binds to the N-terminal part of the protein (Figure 4). E2 enzymes loaded with activated ubiquitin bind to the Rbx domain protein, whereas adaptor/receptor complexes bind specific substrates, which are targets for ubiquitination. The number of cullin scaffolding proteins is comparatively low with three members in $S$. cerevisiae, 
Schizosaccharomyces pombe and A. nidulans, respectively, five in Drosophila melanogaster and in Arabidopsis thaliana and seven members in human (Sarikas et al., 2011).

A well-studied example of CRLs is the SCF (ㅌkp1-ul1-Fbox) ubiquitin ligase complex (Feldman et al., 1997; Hua and Vierstra, 2011; Metzger et al., 2010). It consists of a Cullin1 scaffold protein, which binds the RING domain protein Rbx1 at its C-terminus and interacts with $\underline{\mathrm{S}}$-phase kinase-associated protein 1 (Skp1) adaptor at its N-terminus (Petroski and Deshaies, 2005). The Skp1 adaptor is able to bind Fbox domain containing receptor proteins, which in turn attract specific substrates (Figure 4). The modular structure of SCF complexes is conserved from yeast to humans, which indicates the impact of this ubiquitination pathway (Willems et al., 2004). The complexity of this system is depicted by the number of different Fbox proteins that are recruiters of target proteins: 69 Fbox proteins are identified in humans, approximately 70 in $A$. nidulans and around 700 in A. thaliana (Colabardini et al., 2012; Hotton and Callis, 2008; Hua and Vierstra, 2011). The Fbox domain is highly conserved, located at the $\mathrm{N}$-terminus and interacts with the Skp1 adaptor protein. The C-terminal region of the protein family shows a high level of variability and is responsible for specific substrate binding (Craig and Tyers, 1999).

Active cullins are posttranslationally modified with the ubiquitin-like protein Nedd8 at a conserved lysine residue near its C-terminus (Duda et al., 2008; Wada et al., 1999). Neddylation of cullins induces conformational changes of the Cul1-Rbx1 scaffold, which enables the transfer of the ubiquitin molecule from the E2 enzyme to the target substrate (Duda et al., 2008). The modification of cullins with Nedd8 is a reversible process. Deneddylation reactions are catalyzed by two different isopeptidases that are conserved from fungi to human. The deneddylase $1 / \mathrm{A}$ (Den1/A) catalyzes the removal of Nedd8 from non-cullin proteins in planta (Mergner et al., 2015). The second deneddylase is the constitutive photomorphogenesis complex (COP9 signalosome), which detaches Nedd8 from cullin scaffolding proteins (Beckmann et al., 2015; Christmann et al., 2013; Lyapina et al., 2001; Wu et al., 2003).

Removal of the Nedd8 molecule from cullins, which do not bind a substrate, leads to binding of

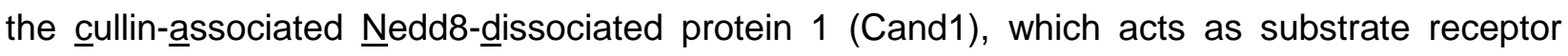
exchange factor, because it hinders the substrate adaptor/receptor complex to interact with the Cullin1 scaffold and inactivates CRLs (Helmstaedt et al., 2011; Goldenberg et al., 2004; Figure 4). The C-terminal part of Cand1 interacts with the $\mathrm{N}$-terminus of cullins and competes with the binding site of the substrate adaptor Skp1, whereas the N-terminal part of Cand1 competes with Nedd8 for the binding site near cullins C-terminus (Goldenberg et al., 2004). The binding of new adaptor/receptor complexes leads to dissociation of Cand1. The cycles of deneddylation and neddylation, for which the order of events is currently not completely 
understood, allow specific binding of different adaptor/receptor complexes and therewith binding of specific substrates, which is essential for a functional UPS. Thereby, deneddylation happens preferably when no substrates are available or bound to the receptor subunit of the CRL (Bosu and Kipreos, 2008; Hua and Vierstra, 2011).

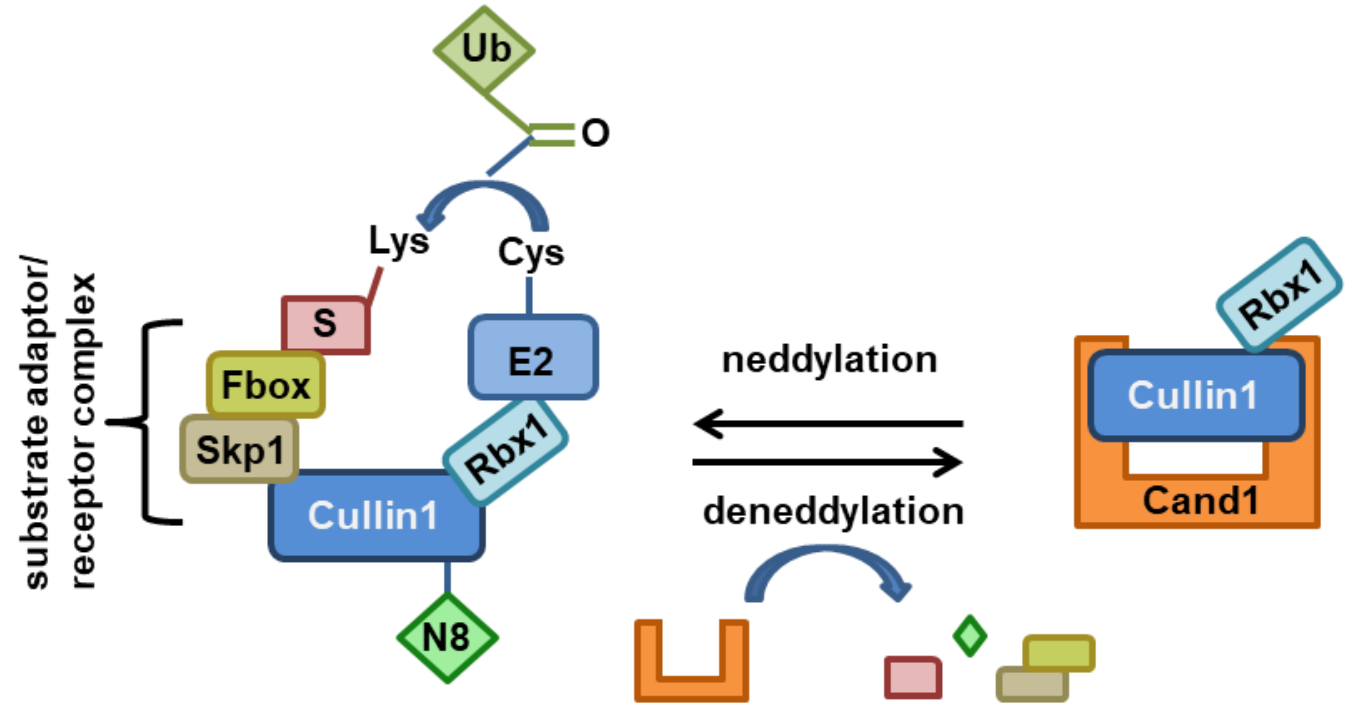

Figure 4: Schematic representation of the modular architecture of eukaryotic SCF E3 ligases.

The Cullin1 scaffold protein binds a RING-box protein (Rbx1) at its C-terminus, which is able to recruit and bind E2 enzymes that are attached to an activated ubiquitin molecule (Ub). The $\mathrm{N}$ terminal part of cullin interacts with the substrate adaptor Skp1, which in turn is able to interact with the huge variety of Fbox protein substrate receptors. Fbox proteins usually recruit substrates (S), which are targets for ubiquitination reactions. If the cullin scaffold protein is modified with the ubiquitin like protein Nedd8 (N8) the SCF complex is active and can transfer the ubiquitin molecule from the E2 enzyme to the target substrate. Deneddylation reactions remove the $\mathrm{N} 8$ and lead to dissociation of the adaptor/receptor complexes from the cullin scaffold. Cand1 acts as substrate receptor exchange factor and competes with cullin binding sites for Nedd8 and Skp1 and can bind to deneddylated cullins.

\subsubsection{COP9 signalosome deneddylates E3 ubiquitin ligases}

The $\underline{\text { COP9 }}$ signalosome (CSN complex) recognizes CRLs, which do not bind a substrate for ubiquitination and inactivates them through the deneddylation reaction. This allows the exchange of substrate receptors what is a prerequisite for controlled protein degradation (Choo et al., 2011; Mosadeghi et al., 2016). The COP9 signalosome was discovered in $A$. thaliana as a repressor of photomorphogenesis (Wei and Chamovitz, 1994). It consists of eight subunits in higher eukaryotes like $H$. sapiens, M. musculus, D. melanogaster and $A$. thaliana. The 
filamentous fungus $A$. nidulans contains all eight subunits, but other fungi like Neurospora crassa or $S$. pombe lack the smallest subunit $\mathrm{CsnH}$. Furthermore, the baker's yeast $S$. cerevisiae harbors a reduced COP9 signalosome complex containing only five core subunits (Braus et al., 2010; Busch et al., 2007; Dubiel et al., 2015; Pick and Pintard, 2009). COP9 signalosome subunits are named Csn1 to Csn8 according to decreasing molecular weight in humans, and CsnA to CsnH in fungi like A. nidulans (Busch et al., 2007; Deng et al., 2000b). Six of the eight subunits carry proteasome, $\underline{\mathrm{COP}}$, eukaryotic initiation factor $3(\mathrm{PCl})$ domains that are important for protein-protein interactions and two subunits contain a $\underline{M} p r 1$ and $\underline{P} a d 1 \underline{N}-$ terminal (MPN) domain (Figure 5) (Scheel and Hofmann, 2005; Wei et al., 2008).

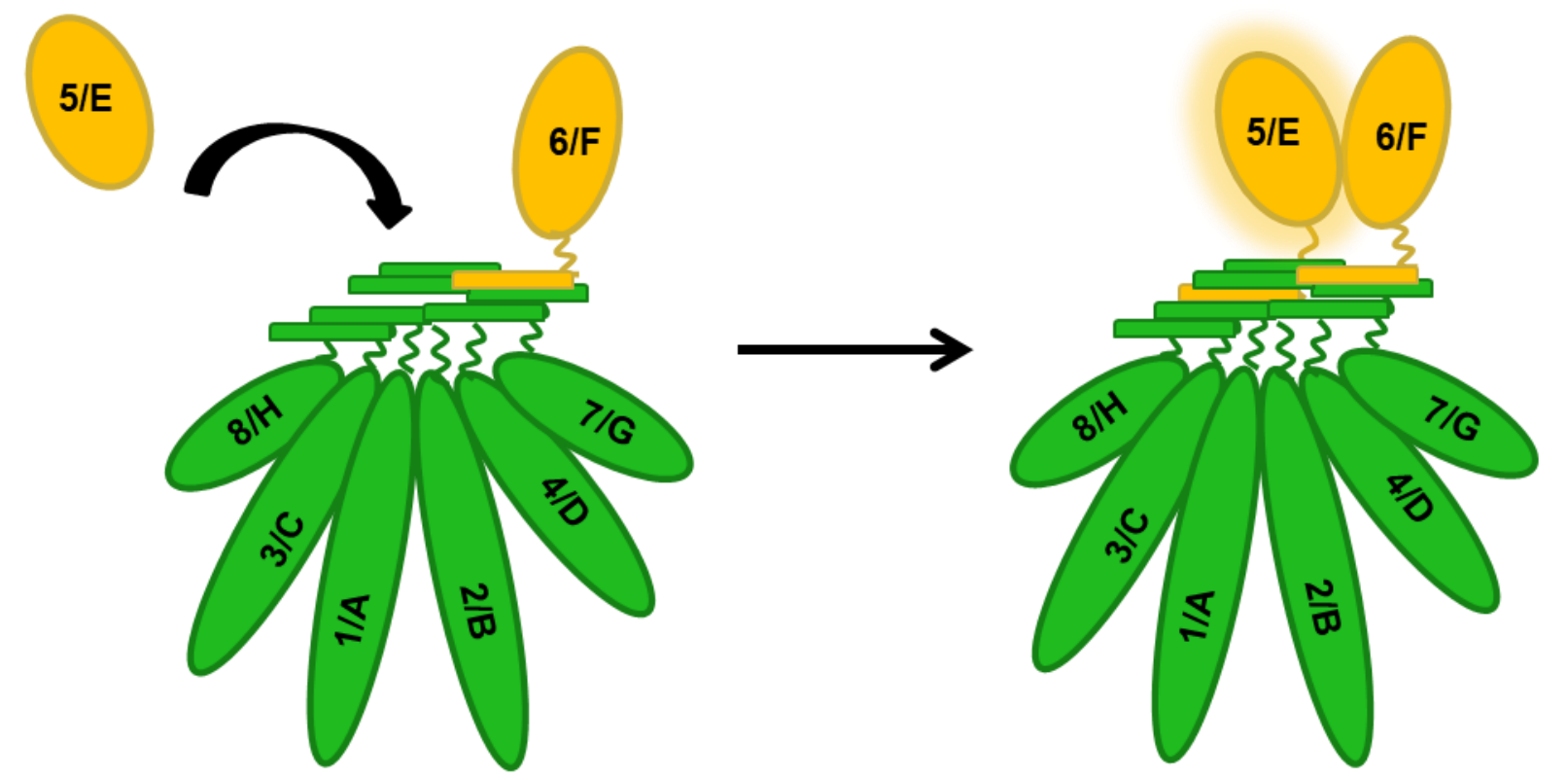

Figure 5: Schematic representation of the COP9 signalosome architecture.

The COP9 signalosome consists of eight subunits that are named according to their size: Csn1/A is the largest, Csn8/H the smallest subunit. Six subunits carry a $\mathrm{PCl}$ domain, have an elongated shape and form a ring like structure. $\mathrm{PCl}$ domain containing subunits are depicted in green. All subunits are connected through a helical bundle formed by C-terminal $\alpha$-helices from all subunits. Two MPN domain containing subunits have rather a globular conformation, which are here depicted in orange. Only Csn5/E contains a JAMM motif, which harbors deneddylation activity when incorporated into the seven-subunit pre-CSN complex.

The COP9 signalosome, the lid of the 26S proteasome (LID) and the eukaryotic initiation factor 3 share high structural similarity and are classified together as "Zomes" (Pick and Pintard, 2009). Each subunit of the COP9 signalosome has a paralogous one in the proteasomal LID. Only one

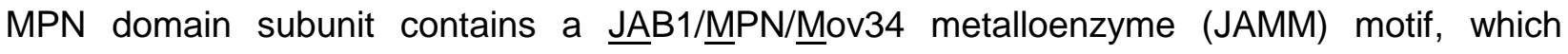


coordinates a zinc atom and confers catalytic activity to the complexes, respectively (Wei et al., 2008). These subunits are Csn5/E in the COP9 signalosome and Rpn11 in the proteasomal LID (Pick and Pintard, 2009). Only a few amino acid changes in the catalytic center shift the substrate specificity from Nedd8 in CsnE to ubiquitin in Rpn11 (Meister et al., 2016). The main function of the COP9 signalosome is the deneddylation of CRLs, whereas the LID of the 26S proteasome is essential for the controlled degradation of ubiquitinated proteins (Braus et al., 2010; Maytal-Kivity et al., 2002; Verma et al., 2002). Both protein complexes are part of the UPS and regulate the degradation of target proteins at different levels.

Lingaraju and co-workers resolved the crystal structure of the human COP9 signalosome at a resolution of $3.8 \AA$ (Lingaraju et al., 2014). The C-terminal helices of all eight subunits are forming a helical bundle. $\mathrm{PCl}$ domain containing subunits form a ring like structure with the two MPN domain containing subunits sitting on top of the helical bundle (Figure 5). Even though the composition of the COP9 signalosome and the proteasomal LID are similar, the complex assembly pathway and catalytic activity differ (Meister et al., 2016). Csn5/CsnE is the last subunit joining the pre-assembled seven-subunit pre-CSN complex in mammals and in A. nidulans, which confers catalytic activity to the complex (Figure 5) (Beckmann et al., 2015; Lingaraju et al., 2014). Conformational changes in the JAMM domain containing subunits (Csn5, Rpn11) take place after incorporation into the multi-subunit complexes. The single JAMM domain containing proteins, which are not incorporated in the complex, show only reduced catalytic activity (Lingaraju et al., 2014; Worden et al., 2014).

Gene deletion of one of the eight encoded csn subunits leads to embryonal lethality in higher eukaryotes (Dohmann et al., 2008; Lykke-Andersen et al., 2003; Oren-Giladi et al., 2008). Filamentous fungi such as $A$. nidulans can vegetatively grow without the COP9 signalosome. A. nidulans mutant strains with defective CSNs revealed a block in sexual development and a disturbed secondary metabolism (Beckmann et al., 2015; Busch et al., 2003, 2007; Mundt et al., 2002). This renders filamentous fungi attractive reference organisms to study functions, assembly and interaction partners of the COP9 signalosome.

\subsubsection{Interacting proteins of the COP9 signalosome}

The best-studied function of the COP9 signalosome is the removal of the posttranslational modifier Nedd8 from cullin scaffold proteins (Beckmann et al., 2015; Cope et al., 2002). The regulation of CRLs by the COP9 signalosome does probably not only rely on its deneddylation activity, but on the recruitment of other proteins that regulate CRL function (Choo et al., 2011). 
Phosphorylation and deubiquitination activity were attributed to the COP9 signalosome due to interactions of the protein complex with kinases or deubiquitinating enzymes (Bech-Otschir et al., 2001; Hetfeld et al., 2005; Naumann et al., 1999; Sun et al., 2002; Zhou et al., 2003). Due to the close connection of the COP9 signalosome to the ubiquitin-proteasome pathway, these phosphorylation and ubiquitination events often affect protein stability.

Kinase activities towards proteins that play a role in signal transduction cascades like c-Jun, IKBa and $\mathrm{p} 150$ (the precursor of NF-KB) were observed by the identification of the COP9 signalosome in HeLa cells (Seeger et al., 1998). The c-Jun transcription factor interacts through Csn5 with the COP9 signalosome and is stabilized by phosphorylation (Naumann et al., 1999; Seeger et al., 1998; Wei et al., 2008). The tumor suppressor protein p53 interacts with Csn5 as well (Bech-Otschir et al., 2001). It becomes phosphorylated at T155 through COP9 signalosome mediated kinase activity in different human cell lines. Phosphorylation destabilizes p53 and sentence the protein for degradation through the UPS (Bech-Otschir et al., 2001; Wei et al., 2008). Kinase activity of purified COP9 signalosome from HeLa or human red blood cells could be attributed to two co-purified kinases: the protein kinase casein kinase 2 (CK2) and the protein kinase D (Uhle et al., 2003). Both kinases were responsible for modification of c-Jun, p53, ІкBa as well as CSN subunits itself (Uhle et al., 2003). Furthermore, the COP9 signalosome copurifies with 1, 3, 4 triphosphate 5/6 kinase (Sun et al., 2002). A direct interaction of this kinase with Csn1 was validated in co-immunoprecipitation experiments (Sun et al., 2002).

Purified COP9 signalosome from mammalian cell lines or $S$. pombe revealed besides kinase, also deubiquitination activity (Zhou et al., 2003). The activity to cleave polyubiquitin chains was attributed to the co-purifying protein Ubp12p in $S$. pombe and to its ortholog Usp15 in humans (Hetfeld et al., 2005; Zhou et al., 2003). Similar to CSN associated phosphorylation activity, deubiquitination activity influences signal transduction pathways like the Wnt/ $\beta$-catenin signaling pathway by regulating stability of proteins (Huang et al., 2009; Wei et al., 2008).

\subsection{Deubiquitinating enzymes reverse the ubiquitination process}

Deubiquitination of proteins counteracts the $\mathrm{CRL}$ activity. The number of different deubiquitinating enzymes (DUBs) in humans is with 84 proteins quite high. Measured at the number of E3 ligases, the total number of DUBs is nearly one magnitude lower (Hutchins et al., 2013). Similar to the components of the ubiquitin-conjugating pathway, the number of E3 ligases or DUBs is correlated to the genome size of different organisms (Hutchins et al., 2013). DUBs

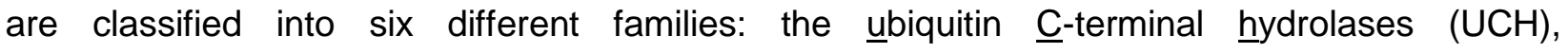


Machado-Joseph domain (Josephin-domain) containing proteases (MJD), ovarian tumor proteases (OTU), ubiquitin-specific proteases (USP), the motif interacting with Ub-containing novel DUB family (MINDY) and JAMM domain metalloproteases. The first five families are cysteine proteases, whereas the JAMM motif containing DUBs are metalloproteases (Hanpude et al., 2015; Komander et al., 2009; Abdul Rehman et al., 2016).

Ubiquitin is not encoded as single gene, but transcribed as linear fusion protein consisting of several ubiquitin ORFs in a row or as fusion to ribosomal proteins (Noventa-Jordão et al., 2000; Özkaynak et al., 1987; Wiborg et al., 1985). DUBs are required to make ubiquitin accessible by cleavage of the fusion proteins (Figure 6, Grou et al., 2015; Özkaynak et al., 1987; Wiborg et al., 1985). Similar to effects of ubiquitination, the removal of the PTM can change function, conformation, activity, stability or localization of target protein. Hence, DUB activity is involved in a number of cellular processes such as proteasomal degradation, endocytosis or immune signaling (Clague et al., 2012; Hicke and Dunn, 2003; Huang et al., 2009; Li et al., 2002; Mukai et al., 2010; Nicassio et al., 2007; Spasser and Brik, 2012; van der Horst et al., 2006). The ubiquitin chain needs to be removed from the protein prior to its degradation by the $26 \mathrm{~S}$ proteasome. The ubiquitin chain is not degraded by the $26 \mathrm{~S}$ proteasome as the unfolding of ubiquitin would require more energy than the cleavage of the ubiquitin chain by DUBs (de Poot et al., 2017; Worden et al., 2017). Additional DUBs are needed to recycle ubiquitin by cleavage of the resulting free ubiquitin chain into monomeric molecules that can be re-used for modification of substrates (Komander et al., 2009). The different functions of DUBs are depicted in Figure 6.

Many DUBs accomplish their function while interacting or being incorporated with or into other complexes (Ventii and Wilkinson, 2009). The proteasomal LID, which is structurally very similar to the COP9 signalosome, harbors a metalloprotease JAMM motif in its catalytically active subunit Rpn11. This deubiquitinase removes ubiquitin chains prior to substrate degradation through the proteasomal core complex when it is incorporated into the 19S regulatory particle (Worden et al., 2017; Yao and Cohen, 2002). Two more DUBs are associated to the 26S proteasome: Usp14 and Uch37 (de Poot et al., 2017). Usp14 preferably deubiquitinates proteins that carry more than one ubiquitin chain (Lee et al., 2016). The function of UCH37 is not well characterized, but it is proposed that it rather removes single ubiquitin moieties from chains than complete ubiquitin chains (Lam et al., 1997; de Poot et al., 2017; Yao et al., 2006). Usp15 deubiquitinates substrates while it interacts with the COP9 signalosome (Hetfeld et al., 2005; Ventii and Wilkinson, 2009; Zhou et al., 2003). 

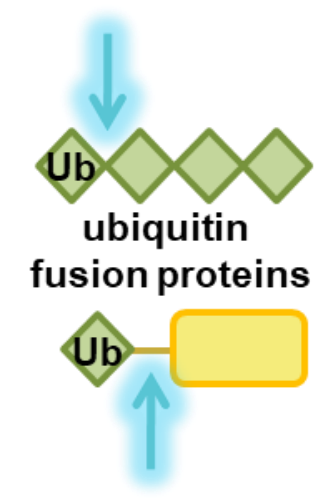

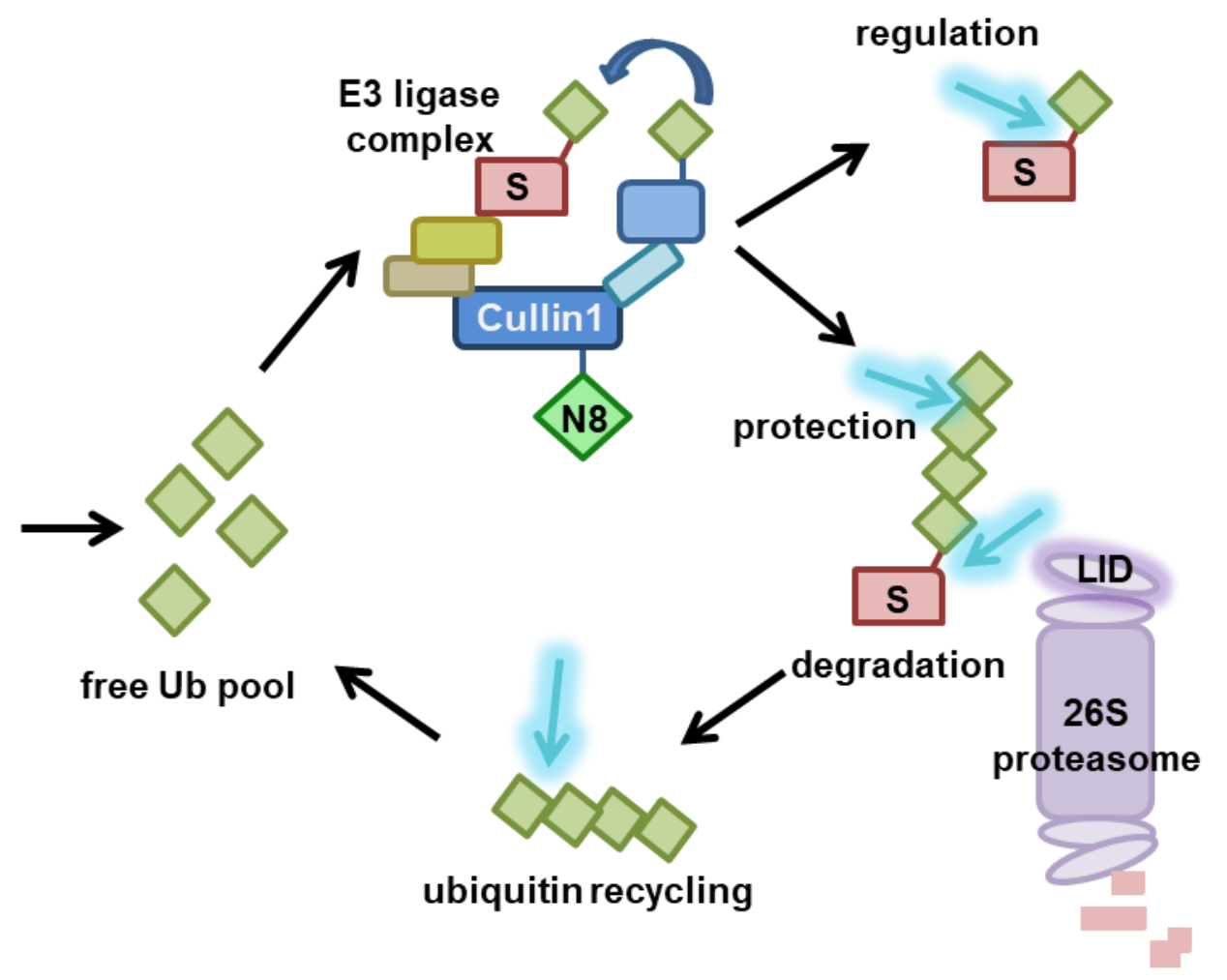

Figure 6: Diverse functions of deubiquitinating enzymes in the ubiquitin cycle.

DUBs provide free ubiquitin $(\mathrm{Ub})$ molecules, which can be used for protein modification, by cleavage of the linear ubiquitin chains or processing of fusion proteins between ubiquitin and certain ribosomal proteins. The free cellular ubiquitin pool is then accessible for the activation by E1 enzymes, which start the ubiquitination cycle. DUBs regulate protein function, localization or conformation by removal of monoubiquitin from substrates (S). Furthermore, they can protect substrates from proteasomal degradation while removing single ubiquitin molecules from the distal end of the ubiquitin chain. DUBs are required for degradation of proteins by the $26 \mathrm{~S}$ proteasome, because they have to remove the ubiquitin chain prior to degradation. This reaction is catalyzed by the intrinsic DUB subunit Rpn11 in the proteasomal LID, but can also be performed by additional proteasome associated DUBs. The cleaved ubiquitin chain needs to be dissected into single ubiquitin molecules to make them again accessible for new ubiquitination events. The blue arrows in the scheme indicate possible actions of different DUBs.

\subsubsection{Ubiquitin-specific proteases}

The largest DUB family are the USPs, which comprise 51 members in humans and 16 in S. cerevisiae (Hutchins et al., 2013). In S. cerevisiae Ubp1 was the first ubiquitin-specific protease that was characterized (Tobias and Varshavsky, 1991). USPs are cysteine proteases and catalyze the hydrolyzation of the isopeptide bond between ubiquitin molecules or between ubiquitin and substrate proteins by their catalytic triad consisting of a cysteine, a histidine and an 
aspartate/asparagine residue (Komander et al., 2009). The catalytic domain comprises approximately 350 amino acids and is located closely to the C-terminal part of the protein (Ye et al., 2009). The catalytic domain can be interrupted by different insertions and can comprise up to 800 amino acids (Ye et al., 2009). Structural analysis of the catalytic domain of human Usp7 and Usp2 revealed a hand like fold with fingers, palm and thumb (Hu et al., 2002; Renatus et al., 2006). Secondary structure predictions of other USPs in other organisms show a conserved pattern of $\alpha$-helices and $\beta$-sheets strongly indicating a conserved fold for USP catalytic domains (Hu et al., 2002; Renatus et al., 2006). Many USP proteins contain at least two ubiquitin binding motifs: one for the distal and one for the proximal ubiquitin. Therefore, they are supposed to cleave preferably linkages between ubiquitin molecules rather than the isopeptide bonds between ubiquitin and the substrate (Ye et al., 2009). A common Cys-X-X-Cys motif was identified in the catalytic USP domain of humans, which was suggested to serve as zinc binding motif (Ye et al., 2009). The ability of zinc binding is shared by approximately $80 \%$ of human and approximately $60 \%$ of all S. cerevisiae USPs (Ye et al., 2009).

The ubiquitin-specific protease Usp15 carries two of these zinc binding motifs and co-purifies with the human COP9 signalosome (Hetfeld et al., 2005). The four cysteine residues comprising the zinc finger motif are located in the catalytic domain in between the residues that represent the catalytic triad. Mutations of only one cysteine codon in the motif revealed an inability of Usp15 to process polyubiquitin chains most probably due to a defect in binding to the ubiquitin chain (Hetfeld et al., 2005). Usp15 shows high sequence similarities to human Usp4 and Usp11, which constitute a small USP subfamily (Baker et al., 1999; Harper et al., 2011). All three proteins share at their $\mathrm{N}$-terminus a domain present in ubiquitin-specific proteases (DUSP) followed by an ubiquitin-like domain (UBL), which are linked through a $\beta$-hairpin structure called DU finger. The function of this domain architecture is currently under investigation, but is speculated to play a role in protein-protein interactions (Harper et al., 2011). Usp4, Usp11 and Usp15 influence among others the transforming growth factor $\underline{\beta}$ (TGF- $\beta$ ) signaling pathway (Aggarwal and Massagué, 2012; Al-Salihi et al., 2012; Clague et al., 2013).

\subsection{The genetic reference organism - Aspergillus nidulans}

Filamentous fungi are characterized by their ability to form large hyphal networks. Most filamentous fungi have a saprobic life style, colonizing in the soil or dead material and thereby playing a role in various carbon cycles. Many plants require fungi for their symbiotic life style or for example as endophytes (Rodriguez et al., 2009). Several fungi are plant pathogens, whereas 
comparably only a small number can cause diseases in mammals. The quite high body temperature of mammals might be a reason for the small number of human fungal pathogens (Casadevall, 2012; Dean et al., 2012). Nevertheless, new pathogens, which are able to infect different hosts, are further evolving (Casadevall et al., 2011).

The filamentous fungus $A$. nidulans belongs to the phylum of Ascomycota. Ascomycetes are classified together with basidiomycetes to the subkingdom Dikarya (greek: di = two, karyon = nucleus) in the kingdom of fungi (Hibbett et al., 2007). Ascomycetes and basidiomycetes differ in the formation of sexual tissues: ascospores are formed in a closed sexual fruiting body (ascus) in ascomycetes, whereas basidiomycetes form sexual spores on top of structures called basidia (Dyer and O'Gorman, 2011; Nwakanma and Unachukwu, 2017). The genus Aspergillus comprises more than 300 different species (de Vries et al., 2017; Samson et al., 2014). Filamentous fungi are secondary metabolites producers. Secondary metabolites are small bioactive molecules that can be useful or harmful for humankind (Gerke and Braus, 2014; Inglis et al., 2013). Aspergilli such as Aspergillus niger or Aspergillus oryzae are utilized in the world's food industry in the fermentation of rice or serve as source of citric acid, respectively (Bennett, 1998). Aspergillus flavus can be problematic for humans as food contaminant or can be causative agent of severe diseases ( $\mathrm{Yu}, 2012$ ). Aspergillus fumigatus is important for biological substrate cycles in the compost, but is also an opportunistic human pathogen. It can cause aspergillosis in immunocompromised patients, often with lethal consequences (Latgé, 1999).

The filamentous fungus $A$. nidulans is a well-established genetic reference organism. Besides the formation of vegetative hyphae, $A$. nidulans is able to enter an asexual life cycle - forming mitotic conidiospores - and a sexual life cycle - forming meiotic ascospores (Adams et al., 1998; Pöggeler et al., 2018). It is a homothallic fungus, meaning that it is self-fertile and does not need a mating partner for sexual reproduction (Braus et al., 2002; Pöggeler et al., 2018). Its genome was fully sequenced in 2005 (Galagan et al., 2005). Gene deletions or mutations can be constructed easily due to its haploid genome and their phenotypical and biochemical analyses revealed immense insights into development, cell cycle, secondary metabolism, signaling cascades, cytoskeleton and pathogenicity of fungi in the past few decades (Gerke et al., 2012; Osmani and Mirabito, 2004; Sarikaya-Bayram et al., 2015; Xiang and Plamann, 2003). 


\subsubsection{Vegetative growth and multicellular reproduction of Aspergillus nidulans}

The growth of $A$. nidulans starts with the germination of an asexual conidiospore or a sexual ascospore, which is triggered by environmental signals. This leads to a network of vegetative hyphae, also called mycelium (Adams et al., 1998; Krijgsheld et al., 2011). The fungal spore generates an axis of polarization, before it forms a germ tube that develops to an elongated tubular structure (hypha). The formation of vegetative hyphae is the simplest form of growth, which allows fast colonization of new environments. Hyphal growth is promoted by apical extension (Harris et al., 2009; Virag et al., 2007). At a certain size, hyphae form septa to divide their cytoplasm into different compartments (Wolkow et al., 1996). Septae are formed by invagination of the plasma membrane and accumulation of cell wall material (Harris, 2001). These so-called cross walls have pores, which allow the transfer of vesicles or nuclei between different compartments. In case of injury or stress, these pores can be closed by Woronin bodies to protect the not affected parts of the hyphae (Collinge and Markham, 1985; Timberlake, 1990). The initial spore can develop more polarity axes and form secondary or tertiary germ tubes (Virag et al., 2007). In addition, hyphae are able to branch and form lateral tubular structures to build a close network (Harris, 2008). Different hyphal branches can fuse to each other to allow intercellular communication and nutrient exchange (Harris, 2008). This vegetative growth form continues in liquid media as long as enough nutrients are present (Krijgsheld et al., 2011). A. nidulans reaches developmental competence after 16-20 hours (h) of growth (Axelrod et al., 1973). The fungus can then sense and react to environmental stimuli such as light, oxygen, temperature or $\mathrm{pH}$ and change gene expression and protein synthesis accordingly during this time (Axelrod et al., 1973; Bayram et al., 2016; Bayram and Braus, 2012).

A. nidulans enters in darkness and on an air-oxygen interface preferably the sexual life cycle, but develops also asexual conidiophores. Illumination induces the asexual conidiophore formation significantly, whereas the energy-consuming sexual life cycle is reduced (Bayram et al., 2016). The decision for the asexual or sexual life cycle is not only dependent on light, but also influenced by environmental signals such as $\mathrm{CO}_{2}, \mathrm{O}_{2}$, nutrients, $\mathrm{pH}$ or internal signals like pheromones (Axelrod et al., 1973; Bayram et al., 2016; Tsitsigiannis et al., 2004, 2005). The fungus enters the asexual life cycle during light exposure resulting in the formation of complex conidiophores, which produce the mitotically derived asexual conidiospores. Already 30 min exposure to light is sufficient to induce the asexual life cycle in fungal hyphae that reached the state of developmental competence (Adams et al., 1998). A full cycle of asexual development can be divided into five stages (Mims et al., 1988). The development of the conidiophore starts at a foot cell, which is a thicker part of the hyphae. A so-called stalk grows vertically out of the 
foot cell to a size of approximately $100 \mu \mathrm{m}$ (Mims et al., 1988). A vesicle is formed at the tip of the stalk, which contains multiple nuclei. Proceeding from this vesicle, small mononuclear compartments, called metulae, are formed by cell budding. On the top of the metulae phialides are formed that in turn develop at their distal end conidiospores through mitotic divisions. The complete conidiophore is built $24 \mathrm{~h}$ after initiation of the foot cell structure (Bayram and Braus, 2012; Calvo et al., 2002; Mims et al., 1988; Timberlake, 1990). The airborne conidiospores are very resistant against environmental stressors and can be easily distributed. Their dark green cell wall pigmentation confers resistance against ultraviolet radiation (Adams et al., 1998; Aramayo et al., 1989; Mayorga and Timberlake, 1990). Mature conidiospores can restart the fungal life cycle (Figure 7).

The sexual life cycle is promoted in the absence of light and under high carbon dioxide pressure. The energy-consuming sexual life cycle results in the formation of a sexual fruiting body (cleistothecium), which is the overwintering structure of the fungus and can survive harsh environmental conditions (Braus et al., 2002; Pöggeler et al., 2018). Sexual development in A. nidulans starts with the fusion of two hyphae by wrapping of one hypha around the other. This results in lumpy hyphal structures (Casselton and Zolan, 2002; Sohn and Yoon, 2002). This nest-like structure, formed by hyphal fusion events, is surrounded by specialized cells. These socalled Hülle cells have nursing and protecting function for the maturing sexual fruiting body (Braus et al., 2002; Sarikaya-Bayram et al., 2010). Nest-like structures are observed approximately $24 \mathrm{~h}$ after initiation of the sexual life cycle (Figure 7). A primordium evolves in the following $24-48 \mathrm{~h}$, which is the immature fruiting body and is characterized by a light reddish cell wall pigmentation (Brakhage, 1998). The immature fruiting body contains so-called ascogenous hyphae, which further develop a sac-like structure termed ascus (Greek: askos = sac): the name-giving structure of ascomycetes (Pöggeler et al., 2018).

The maturation of the cleistothecium is completed after seven days of development. The cell wall of the cleistothecium has a dark pigmentation and is surrounded by Hülle cells (Brown and Salvo, 1994; Sohn and Yoon, 2002). A meiotic nucleus division followed by a mitotic division inside the asci forms eight nuclei. Each nucleus is surrounded by a membrane. A subsequent mitotic nuclear division inside the small single compartments results in binucleate ascospores (Braus et al., 2002; Pöggeler et al., 2018). Bursting of a cleistothecium leads to release of thousands of ascospores that can easily be distributed into the environment. Each spore is able to initiate a new colony undergoing a new life cycle starting with the development of a complex network of vegetative hyphae. 
The development of multicellular structures in filamentous fungi like $A$. nidulans is concomitant with the production of certain secondary metabolites (Bayram and Braus, 2012). Secondary metabolites (SMs) are not essential for fungal growth but confer for example protective function or are important for intercellular communication (Brakhage, 2012). Secondary metabolites are responsible for the pigmentation of the cleistothecium and of the cell wall of the sexual and asexual derived spores (Adams et al., 1998; Brown and Salvo, 1994).

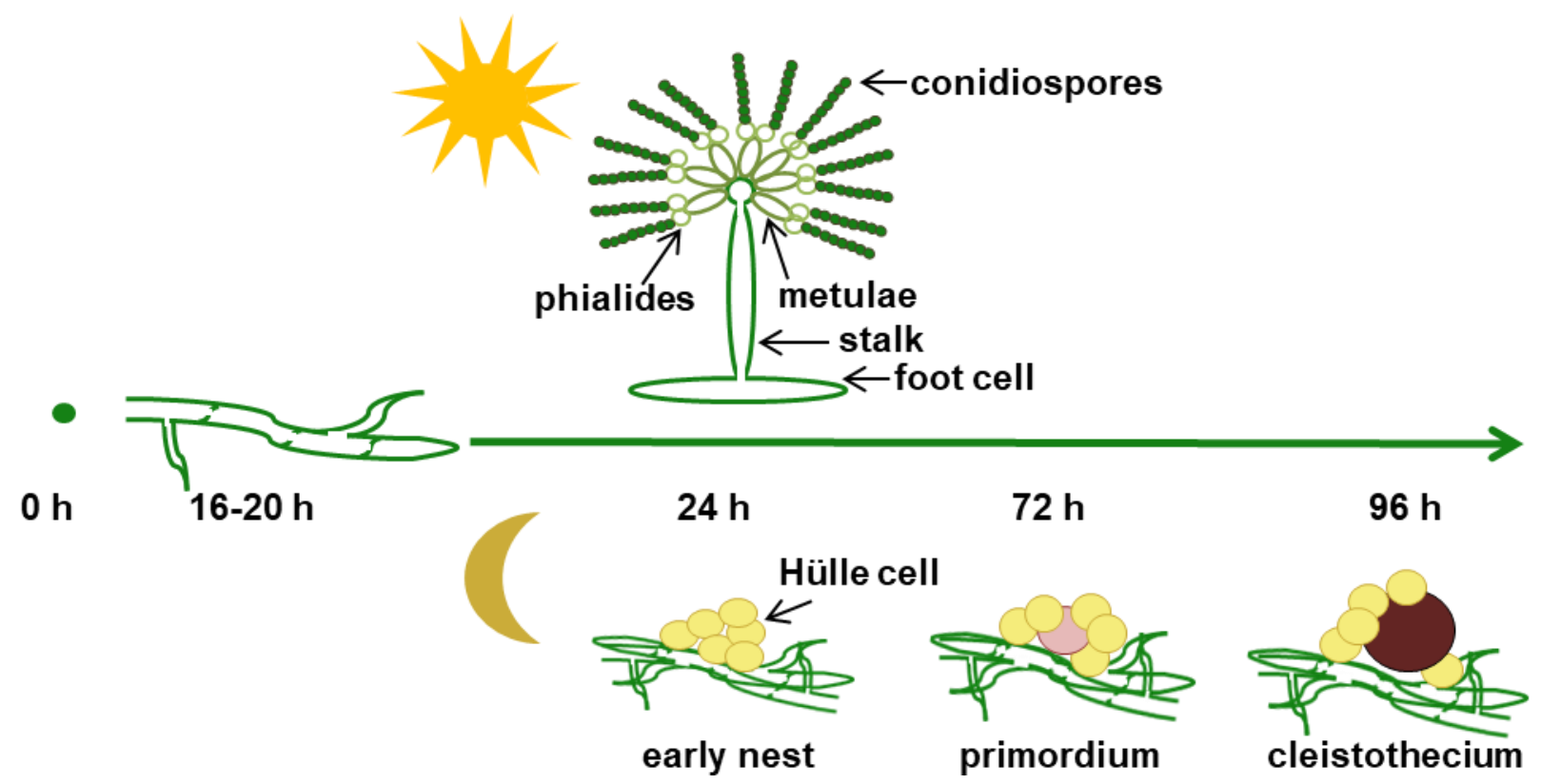

Figure 7: Life cycle of Aspergillus nidulans.

The development starts with a germinating spore, which forms a complex hyphal network. Fungal mycelium reaches developmental competence and can respond to external or internal stimuli by initiation of multicellular development after $16-20 \mathrm{~h}$ of growth. Asexual development, resulting in formation of conidiophores, is favored in light, whereas the more energy-consuming formation of sexual fruiting bodies, called cleistothecia, is preferred in darkness and under high carbon dioxide pressure. Airborne conidiospores are formed by budding of spore forming cells (phialides) and as result of the asexual life cycle. Ascospores are formed inside the asci of cleistothecia. Conidio- and ascospores can re-initiate a fungal life cycle by germination and form vegetative hyphae.

SMs can be of great use for industrial or medical applications, but can also have toxic or carcinogenic impacts on microorganisms, plants and animals (including humans) (Yu and Keller, 2005). Penicillium chrysogenum and $A$. nidulans produce the antibacterial metabolite penicillin, which is one of the most useful secondary metabolites in clinical applications (Brakhage, 1998; 
Hemming, 1944). Other SMs like the family of aflatoxins produced by a number of Aspergilli, such as Aspergillus flavus, have toxic and carcinogenic effects on mammals (Yu, 2012). Inglis and co-workers annotated and identified 71 secondary metabolite gene clusters in $A$. nidulans, from which less than 20 are studied so far (Inglis et al., 2013). Most of these are silent under laboratory growth conditions, which makes the identification and characterization of their products challenging (Sanchez and Wang, 2013).

\subsubsection{Molecular requirements for fungal multicellular development}

The transition of vegetatively grown hyphae to the initiation of multicellular development like asexual conidiophore or sexual cleistothecia formation requires immense changes in the fungal transcriptome as well as proteome. Gene regulation affects not only developmental genes, but also secondary metabolite gene clusters as multicellular development and SM are closely linked in fungi (Bayram et al., 2016; Ruger-Herreros et al., 2011; Timberlake, 1980).

After $A$. nidulans reaches the state of developmental competence, it can react on various external and internal stimuli, which trigger the initiation of multicellular development (Axelrod et al., 1973; Bayram and Braus, 2012). Light is one of the major environmental signals, which has great influence on the life cycle under laboratory growth conditions. Light leads to the repression of sexual fruiting body formation and activation of conidiophore formation (Adams et al., 1998). Four different photoreceptors, termed fungal phytochrome $\underline{A}$ (FphA), light response $\underline{A}$ (LreA), light response $\underline{B}$ (LreB) and cryptochrome $\underline{A}$ (CryA) ensure light sensing (Bayram et al., 2008a; Blumenstein et al., 2005; Purschwitz et al., 2008). These fungal light sensors are specialized for light absorption of certain wavelengths. FphA was identified as red-light sensor, whereas LreA and LreB sense blue light (Purschwitz et al., 2008). LreA and LreB are the orthologs of the wellstudied White $\underline{\text { Collar }}$ (WC) proteins 1 and 2 in N. crassa, which are transcription factors that form a blue light sensing complex (Ballario et al., 1996; Froehlich et al., 2002; Harding and Melles, 1983; Linden and Macino, 1997; Purschwitz et al., 2008). The fungal CryA protein has a combined light sensing and photolyase activity. The absorption spectrum encompasses blue and UV-A light (Bayram et al., 2008a). Defects in these receptors lead to disturbed developmental processes. The blue light receptors LreA and LreB are suggested to be activators of the sexual life cycle and FphA represses their function during growth in light (Purschwitz et al., 2008). Exposure of developmental competent mycelia to white light for $30 \mathrm{~min}$ revealed differential expression of approximately 500 genes in A. nidulans (Ruger-Herreros et al., 2011). 
The gene with the highest light-induced downregulation encodes the velvet $\underline{A}$ protein $(\mathrm{VeA})$ (Ruger-Herreros et al., 2011). The VeA protein activates the sexual life cycle (Kim et al., 2002). It interacts with phytochrome FphA to respond to external light signals. FphA interacts with a complex of the blue light receptors LreA and LreB. All four proteins together form a light-sensing complex (Bayram et al., 2010; Purschwitz et al., 2008). A recent study showed that $19 \%$ of all transcribed genes in $A$. nidulans are dependent on light (Bayram et al., 2016). Expression of the

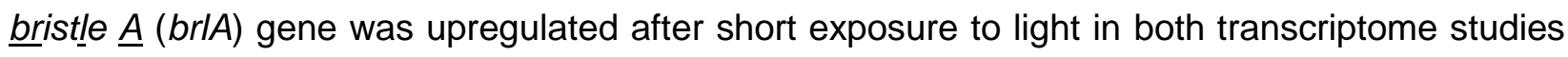
(Bayram et al., 2016; Ruger-Herreros et al., 2011). It encodes a transcription factor, which is essential for vesicle formation during conidiophore development (Adams et al., 1988; Clutterbuck, 1969; Mooney and Yager, 1990).

A special combination of environmental conditions, such as light, $\mathrm{pH}$, temperature or the presence of mating partners, is required for initiation of the fungal sexual life cycle. Under laboratory growth conditions (stable temperature, $\mathrm{pH}$, nutrients), the absence of light or a disturbed light sensing mechanism leads to the initiation of the sexual life cycle (Adams et al., 1998). The velvet domain containing protein $\mathrm{VeA}$ is involved in this light-dependent decision of fungal development (see Chapter 1.3.1) (Mooney and Yager, 1990; Pöggeler et al., 2018). Furthermore, internal stimuli trigger the choice of the development path. The concentration of hormone-like signaling molecules composed of unsaturated fatty acid, so-called precocious sexual inducer (psi) factors, is crucial (Tsitsigiannis et al., 2004, 2005). Comparably high amounts of these oxylipins are produced in the initiation phase of sexual development (Bayram et al., 2016). ppoA encodes one of three psi factor biosynthesis genes and its expression is positively regulated by the master regulator of fungal development and secondary metabolism VeA (Bayram and Braus, 2012; Tsitsigiannis et al., 2004).

\subsection{Regulation of development by protein complexes}

\subsubsection{Velvet proteins - major regulators of fungal development}

Analyses of $A$. nidulans mutant strains derived from exposure to $\mathrm{X}$-ray radiation lead to the identification of the veA gene locus. Mutations in this locus (veA1 mutation) increased the amount of conidiospore formation compared to wild type strains (Käfer, 1965). The point mutation in veA1 strains is attributed to the start codon "ATG", in which the guanine (G) is mutated to a thymine $(T)$. This leads to a displaced transcription start at the second ATG in the ORF, which results in a 36 amino acids N-terminally truncated VeA protein, termed VeA1 (Kim et al., 2002). The veA1 mutation leads to increased conidiospore production not only in light, but 
especially in darkness, where the wild type $(v e A+)$ forms preferentially cleistothecia and decreased numbers of conidiophores. Sexual fruiting body formation is strongly reduced in veA1 mutants as well. Deletion of the full veA ORF leads to a complete inability to form cleistothecia. Therefore, VeA is considered as positive regulator of sexual development (Kim et al., 2002). The protein encoded by the wild type veA ORF comprises 573 amino acids. VeA contains a nuclear localization signal (NLS) close to its N-terminal part, which is partially lost in the VeA1 mutation. This indicates already that the nuclear localization of $\mathrm{VeA}$ is important for the correct progression of the sexual life cycle (Bayram and Braus, 2012). A PEST domain is located close to the Cterminus. PEST domains are rich in amino acids like proline $(\underline{P})$, glutamic acid $(\underline{E})$, serine $(\underline{S})$ and threonine $(\underline{T})$ and are often found in proteins, which have a short half-life (Rogers et al., 1986). The increased serine and threonine residues are phosphorylation targets, which modifications might be a signal for degradation by the $26 \mathrm{~S}$ proteasome (see Chapter 1.1.2) (Bayram and Braus, 2012; Glickman and Ciechanover, 2002).

VeA is one of four $A$. nidulans proteins, which contain a characteristic velvet domain of approximately 150 amino acids. The other members are named velvet-like $\underline{B}$ (VelB), viability of spores $\underline{A}($ VosA) and velvet-like $\underline{\mathrm{C}}$ (VelC) (Bayram and Braus, 2012). All of them are well conserved in the fungal kingdom (Bayram and Braus, 2012). Velvet proteins form different homo-, hetero- or multimeric complexes that regulate development and secondary metabolism in different ways (Park et al., 2012; Sarikaya-Bayram et al., 2014, 2015). VeA forms a complex with VelB in the cytoplasm and they are transported together into the nucleus by the help of the nuclear a importin, KapA (Figure 8, Bayram et al., 2008b; Stinnett et al., 2007). The nuclear import of the protein complex is dependent on several factors. Light decreases nuclear accumulation of VeA (Bayram et al., 2008b). The LaeA-like methyltransferase $\underline{F}$ (LImF) interacts with VeA in the cytoplasm and controls its localization (Palmer et al., 2013). Nuclear accumulation of $\mathrm{VeA}$ is slightly increased in $\triangle I / m F$, whereas overexpression (OE) of IImF blocks nuclear accumulation (Palmer et al., 2013). Additionally, another protein complex consisting of the $\underline{V}$ A interacting protein $\underline{\mathrm{C}}(\mathrm{VipC})$, VipC associated protein $\underline{B}$ (VapB) and $\underline{\text { VipC }}$ associated protein $\underline{A}(\mathrm{VapA})$ influences fungal development by regulating the subcellular localization of VeA (Sarikaya-Bayram et al., 2014). The formation of this protein complex is independent on light conditions. VapA is a membrane bound zinc finger containing protein, which interacts at the plasma membrane with the two methyltransferases VapB and VipC. The methyltransferases VapB and VipC dissociate from the plasma membrane and enter the nucleus after perception of certain environmental stimuli (Figure 8, Sarikaya-Bayram et al., 2014, 2015). 


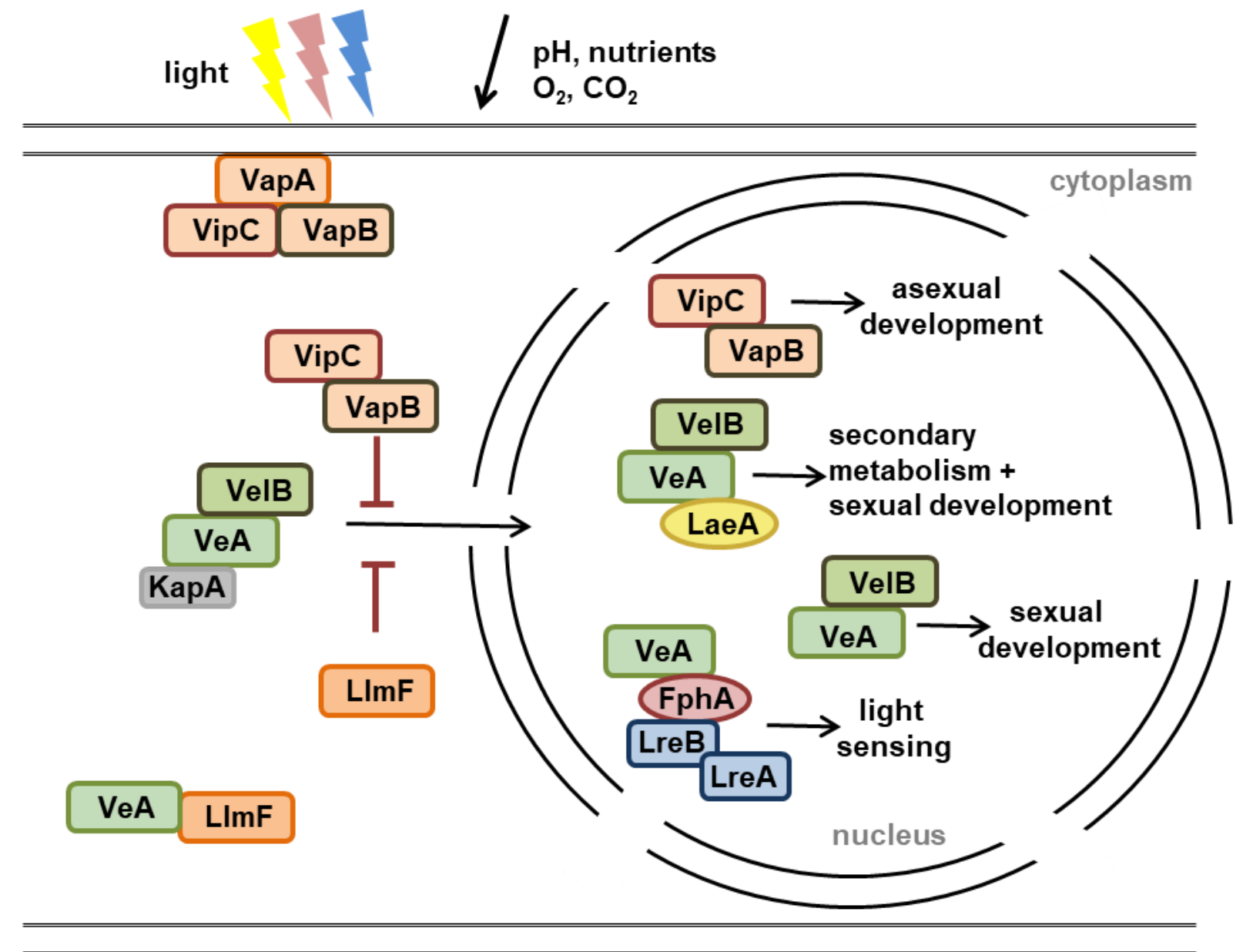

Figure 8: Localization and interaction partners of $\mathrm{VeA}$, the master regulator of development and secondary metabolism.

The velvet domain containing protein VeA forms a complex with VelB and both proteins migrate together into the nucleus. The nuclear localization of the VeA-VelB heterodimer is dependent on environmental stimuli such as light, $\mathrm{pH}$, oxygen or carbon dioxide concentration. Furthermore, it is regulated by different methyltransferases (LImF or VipC-VapB complex), which oppose the nuclear accumulation of $\mathrm{VeA}$. Once the $\mathrm{VeA}-\mathrm{VelB}$ heterodimer reaches the nucleus, it induces the sexual life cycle and can form different protein complexes that regulate fungal development. The most prominent one is the velvet complex, consisting of VeA-VelB and the methyltransferase LaeA. This complex is a master regulator of fungal secondary metabolism and sexual development. VeA can interact with the photoreceptor complex and plays therewith a role in light sensing. The methyltransferases $\mathrm{VapB}$ and $\mathrm{VipC}$ can regulate gene expression inside the nucleus and thereby promote asexual and repress sexual development (adapted from SarikayaBayram et al., 2015).

Cytoplasmic VapB and VipC hinder the nuclear entry of VeA. Interactions of VeA with VipCVapB complex are localized to the nuclear periphery and to the nucleus itself (Sarikaya-Bayram 
et al., 2014, 2015). The phosphorylation state of different amino acid residues in VeA influence the interaction with VelB and the nuclear import of the VeA-VelB protein complex (Rauscher et al., 2016). The VelB-VeA heterodimer associates with the constitutively nuclear methyltransferase lack of $\underline{a} f I R$ expression $\underline{A}$ (LaeA), where VeA serves as bridging factor between VelB and LaeA and they form the so-called velvet complex (Figure 8, Bayram et al., 2008b; Bok et al., 2006; Bok and Keller, 2004). LaeA is a regulator of secondary metabolism and required for sterigmatocystin or penicillin biosynthesis in A. nidulans (Brakhage, 2012). The LaeA ortholog in the close relative $A$. fumigatus is required for the expression of the gliotoxin gene cluster (Bok et al., 2006; Bok and Keller, 2004). Besides the velvet complex, other homoand heterodimers of velvet domain containing proteins are formed inside the nucleus. The heterodimer of VelB and VosA is responsible for spore viability and trehalose biogenesis (Park et al., 2012). Furthermore, interactions of VeA with VipC occur in the nucleus, but their exact function in fungal development is still a matter of investigation (Sarikaya-Bayram et al., 2014).

The velvet domain comprises 150 amino acids and contains no described sequence motifs (Bayram and Braus, 2012). It serves as protein-protein interaction domain and different protein complexes between velvet proteins have been identified (Bayram and Braus, 2012). It was shown in 2013 that the velvet domain of VosA is able to bind to DNA (Ahmed et al., 2013). Furthermore, crystallization of the velvet domain revealed striking similarity to the Rel-homology domain of the mammalian nuclear factor kappa-light-chain-enhancer of activated $\underline{B}$-cells (NF-kB) family transcription factors (Ahmed et al., 2013). There are five proteins belonging to the NF-KB family: p65 (RelA), RelB, c-Rel, p105/p50 (NF-kB1) and p100/52 (NF-kB2) (Oeckinghaus and Ghosh, 2009). They are able to interact with each other and form different homo- or heterodimeric complexes that differ in their function, similar to the family of velvet proteins in filamentous fungi (Bayram and Braus, 2012; Oeckinghaus and Ghosh, 2009). In non-stimulated cells, the NF-KB transcription factors are localized in the cytoplasm and form complexes with their inhibitor of $\underline{\mathrm{kB}}$ proteins $(\mathrm{I} \mathrm{KB})$. The inhibitor proteins become phosphorylated due to different stimuli at their PEST domain and are subsequently degraded by the ubiquitin-proteasome system (Oeckinghaus and Ghosh, 2009). The IkBa becomes phosphorylated after treatment with cytokines that are involved in inflammation reactions and therewith it becomes a substrate for the UPS (Schweitzer et al., 2007). The NF-kB transcription factor can subsequently move to the nucleus to induce transcription of target genes. Binding of $І \mathrm{KBa}$ to the COP9 signalosome leads to stabilization and re-accumulation of the inhibitor by deubiquitination reactions catalyzed by Usp15. The stabilization of IKBa leads to the inhibition of NF-KB transcription factor activity (Schweitzer et al., 2007). 
The transcription factors of the NF-KB family act in mammalian signaling pathways, which are connected to apoptosis or to the immune system (Oeckinghaus and Ghosh, 2009; Sun and Andersson, 2002). Similarly, the fungal velvet protein family coordinates development and concomitant secondary metabolism, which is crucial for survival or for defense against predators (Bayram and Braus, 2012). Despite the low sequence similarity of only $13 \%$ a common structure and conserved residues in the DNA binding domain indicate the existence of a common ancestor of both protein families (Ahmed et al., 2013; Gerke and Braus, 2014).

\subsubsection{The COP9 signalosome in fungal development and secondary metabolism}

A functional COP9 signalosome is essential for regulation of CRL activity through deneddylation, which in turn regulates protein half-life control. COP9 signalosome dysfunction has been attributed to drastic developmental defects and diseases in higher eukaryotes (Lykke-Andersen et al., 2003; Oren-Giladi et al., 2008).

Deletion of any of the eight CSN subunit encoding genes in $A$. nidulans leads to the same phenotype: a block in sexual fruiting body formation and an altered secondary metabolism represented by accumulation of red color in hyphae and surrounding media (Beckmann et al., 2015; Busch et al., 2003, 2007). Despite their inability of sexual fruiting body formation, csn mutants initiate the sexual life cycle independently from light (Busch et al., 2003). Defects in the circadian rhythm of $N$. crassa were observed in csn-2 mutant strains that indicate a function of CSN in light depending developmental control due to stability regulation of proteins regulating light responses (He et al., 2005). Each single subunit of the A. nidulans COP9 signalosome is required for the deneddylation activity towards CRLs, whereas only CsnE can catalyze the deneddylation reaction. Neddylated CullinA proteins accumulate in csn deletion strains (Beckmann et al., 2015). In tandem affinity purifications (TAP) with the fungal Nedd8 ortholog (NeddH) Fbox 1, 2, 15 and 23 bound to SCF complexes were identified in $\triangle c s n E$ strains (von Zeska Kress et al., 2012). The COP9 signalosome counteracts the accumulation of CRL complexes containing these Fbox proteins. A functional COP9 signalosome stabilizes protein levels of Fbox15 (von Zeska Kress et al., 2012).

The effect of dysfunctional COP9 signalosome was analyzed on a transcriptomic and metabolomic level with an $A$. nidulans csnE deletion strain during different developmental stages (Nahlik et al., 2010). $15 \%$ of the fungal genome are differentially regulated in $\triangle c s n E$, whereas the regulated genes differ between vegetative growth and multicellular development (Nahlik et al., 2010). In the scope of a metabolome study comparing $A$. nidulans wild type and $\Delta c s n E$ the 
red color causing compounds in $\triangle c s n E$ were identified as orsellinic acid (ors) and its derivatives (Nahlik et al., 2010). Accordingly, genes belonging to the ors gene cluster ors $B$ or ors $C$ were upregulated during multicellular fungal development in $\Delta c s n E$ (Nahlik et al., 2010).

Development and the production of secondary metabolites are closely linked in fungi (Brakhage, 2012). Disruption of a functional ubiquitin-proteasome system through deletion of CsnE encoding gene leads to the upregulation of different SM clusters (Gerke et al., 2012). The identification of the derivative of $\underline{b}$ enzaldehyde ( $d b a)$ gene cluster producing 2,4-dihydroxy-3 - methyl-6-(2-oxopropyl) benzaldehyde (DHMBA), a molecule with antibiotic function, was possible through this approach (Gerke et al., 2012).

\subsubsection{Deubiquitinating enzymes in fungi}

The ubiquitination cascade within the ubiquitin-proteasome system is highly conserved from fungi to humans (Nandi et al., 2006). This suggests that the reverse deubiquitination process also shows similarities. Initially, 17 DUB encoding genes were identified in the genome of S. cerevisiae (Amerik et al., 2000). Translated proteins encompass 16 members of the USP family and one of the $\mathrm{UCH}$ family. Gene deletions of all single genes did not show any strong developmental defect (Amerik et al., 2000). A more recent study identified 24 DUB proteins in yeast, adding four members to the JAMM metalloprotease DUB family and one protein to the OTU family (Hutchins et al., 2013). The family of MJD proteases is absent in yeast. Gene deletions lead only to minor developmental defects (Amerik et al., 2000; Hutchins et al., 2013). S. pombe expresses 27 DUBs and the fungus $N$. crassa contains 29 DUBs (Hutchins et al., 2013). For all named fungi, the family of ubiquitin-specific proteases comprises the most members (Hutchins et al., 2013). The best studied DUB in S. cerevisiae is named Doa4, which belongs to the JAMM family and does not have an ortholog in humans. Doa4 co-purifies with the 26S proteasome and recycles ubiquitin from substrates, which are targeted to proteasomal degradation. doa4 deletion strains contain a depleted pool of free ubiquitin in the cells (Papa et al., 1999; Swaminathan et al., 1999). Global analysis of S. pombe DUBs revealed co-purification with macromolecular protein complexes like 19S regulatory particle of the $26 \mathrm{~S}$ proteasome, the

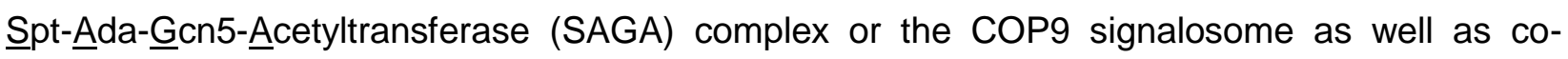
purification with smaller complexes like the Cdc48 ATPase complex (Kouranti et al., 2010; Zhou et al., 2003). Up to date, only one deubiquitinating enzyme was characterized in the filamentous ascomycete $A$. nidulans, which plays a role in the carbon catabolite repression (CCR) pathway. Four proteins, CreA-CreD, regulate the CCR system (Alam and Kelly, 2017). The protein CreB 
belongs to the USP family and carries the conserved cysteine, histidine and aspartate residues. Concomitant with the domain architecture, deubiquitination activity was attributed to CreB (Lockington and Kelly, 2001). Bioinformatical and genetical analyses of DUBs like in S. cerevisiae or S. pombe are lacking in A. nidulans.

\subsection{Aims of the study}

In this study two open questions were addressed, which aim to understand the contribution of controlled specific protein degradation in the potential to perform developmental programs linked to secondary metabolism in the filamentous fungus Aspergillus nidulans.

The extents of the proteome changes in a vegetatively grown mutant fungus were determined, which is impaired in the specific protein degradation control. In this mutant strain the COP9 signalosome, which allows exchanging the substrate receptors of E3 ubiquitin ligases, is not functional. Therefore, stable isotope labeling with amino acids in cell culture (SILAC) was adapted to the use for the filamentous ascomycete $A$. nidulans. This method enables the quantification of proteome changes in different strains by labeling cultures with different isotopes of L-lysine or L-arginine amino acids. In this study, the proteome of $\Delta c s n E$ to wild type and complementation strains was compared during the state of fungal developmental competence.

The ubiquitination of substrates by E3 ubiquitin ligases can be reversed by deubiquitinating enzymes (DUBs). The impact of a COP9 signalosome interacting UspA deubiquitinase on the fine tuning of fungal differentiation, secondary metabolism and the molecular basis of its cellular function was examined during this study. The interaction of UspA with single COP9 signalosome subunits was analyzed with yeast-two-hybrid assays, BiFC and co-localization experiments. Furthermore, the connection of the proteins on a transcriptional level was analyzed. Catalytic activity of UspA was confirmed through deubiquitination assays. Substrates for UspA deubiquitination reactions are presumably less stable in the absence of this enzyme. Fusion protein abundance of different GFP-tagged velvet domain containing proteins and the catalytically active subunit CsnE of the COP9 signalosome were investigated during fungal development. Additionally, putative interaction partners and therewith potential substrates were explored using GFP pull down experiments with a functional UspA-GFP and an inactive UspA-GFP variant, in which two for catalytic activity essential cysteine residues were exchanged to alanine. 


\section{Material and Methods}

\subsection{Material and Chemicals}

Media for strain cultivation, buffers and solutions were prepared with products from APPLICHEM GMBH (Darmstadt, Germany), BD Biosciences (Heidelberg, Germany), CARL ROTH GMBH \& Co. KG (Karlsruhe, Germany), FLUKA (Neu-Ulm, Germany), INVITROGEN (Carlsbad, CA, USA), MERCK KGAA (Darmstadt, Germany), BIOZYME SCIENTIFIC GMBH (Hessisch Oldendorf, Germany), Roche Diagnostics GmbH (Mannheim, Germany), Sigma-AldRich Chemie GmbH (München, Germany), Serva Electrophoresis GmBH (Heidelberg, Germany) and OxOID DEUTSCHLAND GMBH (Wesel, Germany).

Plastic consumables such as pipet tips, petri dishes, reaction tubes etc. were purchased from SARStedt AG \& Co. (Nümbrecht, Germany), StARLAB GmBH (Hamburg, Germany), NERBE PLUS GMBH (Winsen/Luhe, Germany) and EPPENDORF AG (Hamburg, Germany).

PCR cyclers from T Professional Standard 96, T Professional Trio 48 and T Professional Standard 96 Gradient thermocyclers from BIOMETRA GMBH (Göttingen, Germany) and Primus 96 Thermal Cyclers from MWG BIOTECH AG (Ebersberg, Germany) were used. NanoDrop ND-1000 photospectrometer from PEQLAB BIOTECHNOLOGIE GMBH (Erlangen, Germany) was used for determination of concentration of DNA, RNA or protein samples. Quantitative real time PCRs were performed with a CFX Connect ${ }^{\mathrm{TM}}$ Real-Time System purchased from BIORAD (München, Germany). The SpeedVac concentrator from THERMO FISHER SCIENTIFIC (Waltham, MA, USA) was used. Agarose gel electrophoresis was performed with Mini-Sub ${ }^{\circledR}$ Cell GT chambers and the PowerPac ${ }^{\mathrm{TM}} 300$ power supply and SDS-polyacrylamide gel electrophoresis were performed with the Mini-Protean ${ }^{\circledR}$ Tetra Cell, Mini Trans-Blot $^{\circledR}$ Electrophoretic Cell and powered with the PowerPac $^{\text {TM }} 3000$ from BIO-RAD LABORATORIES (Hercules, CA, USA). Proteins were transferred from SDS-polyacrylamide gels onto Amersham ${ }^{\mathrm{TM}} \operatorname{Protran}^{\mathrm{TM}} 0.45 \mu \mathrm{m}$ NC nitrocellulose blotting membranes and DNA was blotted to Amersham ${ }^{\mathrm{TM}}$ Hybond- $\mathrm{N}^{\mathrm{TM}}$ nylon membranes from GE HEALTHCARE (Little Chalfont, United Kingdom). Chemiluminescence was detected by exposure of the membranes with the Fusion SL chemiluminescence detector from PEQLAB GMBH (Erlangen, Germany). For centrifugation of 1.5 and $2 \mathrm{ml}$ reaction tubes, Biofuge fresco (cooled) and Biofuge pico centrifuges from HeRAEUS INSTRUMENTS GMBH (Hanau, Germany) were used. For centrifugation of 10,15 and $50 \mathrm{ml}$ centrifuge tubes Rotixa/RP from ANDREAS HETTICH GMBH \& Co. KG (Tuttlingen, Germany), 5804R from EPPENDORF AG (Hamburg, Germany) and 4K15C 
from Sigma LABORZENTRIFUGEN GMBH (Osterode am Harz, Germany) were used. For pH determination a WTW bench pH/mV Routine meter pH 526 (SIGMA-ALDRICH) was used. Polymerases and restriction enzymes were obtained from THERMO FISHER SCIENTIFIC (Schwerte, Germany), trypsin was purchased from SERVA ELECTROPHORESIS GMBH (Heidelberg, Germany). Primers were obtained from EUROFINS GENOMICS GMBH (Ebersberg, Germany). The GeneRuler $1 \mathrm{~kb}$ DNA ladder and the PageRuler ${ }^{\mathrm{TM}}$ Prestained Protein Ladder from THERMO FISHER SCIENTIFIC (Schwerte, Germany) were used for DNA and protein on-gel band size determination. Ampicillin purchased from CARL ROTH GMBH \& CO. KG (Karlsruhe, Germany), pyrithiamine hydrobromide from SIGMA-ALDRICH (München, Germany), clonNAT (nourseothricin dihydrogen sulfate) from WERNER BIOAGENTS (Jena, Germany) and phleomycin from INVIVOGEN (San Diego, CA, USA) were used for selection of microorganisms.

\subsection{Media and growth conditions}

Liquid and solid media were dissolved in $\mathrm{dH}_{2} \mathrm{O}$ and sterilized at $120^{\circ} \mathrm{C}$ for 20 min at 2 bar if not indicated otherwise. Heat-sensitive compounds were sterile filtrated and added to the medium after autoclaving.

\subsubsection{Bacterial growth conditions}

E. coli strains DH5a (Grant et al., 1990) were cultivated in lysogeny broth (LB) medium containing $1 \%(\mathrm{w} / \mathrm{v})$ tryptone, $0.5 \%$ (w/v) yeast extract, $1 \%(\mathrm{w} / \mathrm{v}) \mathrm{NaCl}$ (Bertani, 1951). For solid plates $2 \%$ (w/v) agar was added. $100 \mu \mathrm{g} / \mathrm{ml}$ ampicillin final concentration was used as selection marker.

\subsubsection{Aspergillus nidulans growth conditions}

A. nidulans wild type strain AGB552 (veAt) was used as background for SILAC strains, and AGB551 (veAt) was used as wild type for all other experiments (Bayram et al., 2012). Strains were grown in minimal medium (MM) consisting of $1 \%(\mathrm{w} / \mathrm{v})$ glucose, $1 \mathrm{x}$ AspA $(7 \mathrm{mM} \mathrm{KCl}$, $\left.70 \mathrm{mM} \mathrm{NaNO}_{3}, 11.2 \mathrm{mM} \mathrm{KH}_{2} \mathrm{PO}_{4}, \mathrm{pH} 5.5\right), 2 \mathrm{mM} \mathrm{MgSO}_{4}, 0.1 \%(\mathrm{v} / \mathrm{v})$ trace element solution $\left(76 \mu \mathrm{M} \mathrm{ZnSO}_{4}, 178 \mu \mathrm{M} \mathrm{H}_{3} \mathrm{BO}_{4}, 25 \mu \mathrm{MnCl}_{2}, 18 \mu \mathrm{M} \mathrm{FeSO}_{4}, 7.1 \mu \mathrm{M} \mathrm{CoCl}_{2}, 6.4 \mu \mathrm{M} \mathrm{CuSO}\right.$, 6.2 $\mu \mathrm{M} \mathrm{Na}_{2} \mathrm{MoO}_{4}, 174 \mu \mathrm{M}$ EDTA) pH 5.5 (Käfer, 1977). 2 \% (w/v) agar was added for solid agar plates and, if necessary, $0.1 \%(\mathrm{w} / \mathrm{v})$ uracil. The minimal medium was supplemented according to specific strain requirements with $0.1 \%(\mathrm{v} / \mathrm{v})$ pyridoxine, $5 \mathrm{mM}$ uridine or $1 \mu \mathrm{g} / \mathrm{ml}$ 
4-aminobenozoic acid (PABA). For strain selection $10 \mu \mathrm{g} / \mathrm{ml}$ phleomycin or $120 \mathrm{ng} / \mathrm{ml}$ nourseothricin was added to the medium. The growth of the SILAC strains was promoted by the supplementation with $0.75 \mathrm{mM}$ L-lysine or $10 \mathrm{mM} \mathrm{L-arginine}(\mathrm{pH} 8.5)$, respectively.

Liquid cultures of $A$. nidulans were incubated for $20 \mathrm{~h}$ at $37^{\circ} \mathrm{C}$ under agitation. Flasks with indentations were used to provide enough oxygen required for fungal vegetative development. Agar plates for generating conidiospores for further experiments were incubated for three to five days (d) at $37^{\circ} \mathrm{C}$. Conidiospores were harvested in $\mathrm{NaCl}$-Tween $(0.96 \%$ (w/v) NaCl, $0.01 \%(\mathrm{v} / \mathrm{v})$ Tween 80 (Polyoxyethylene sorbitan monooleate)) and stored at $4^{\circ} \mathrm{C}$. Asexual or sexual development was induced by growing fungal strains on agar plates and incubating them under constant illumination or in darkness under oxygen limiting conditions for three to five (asexual) or seven to fourteen days (sexual), respectively. Sexually grown plates were sealed with Parafilm ${ }^{\circledR} \mathrm{M}$ (MERCK, Darmstadt, Germany) to reduce oxygen supply. For protein or RNA extraction during multicellular development, vegetative grown mycelia from liquid cultures were shifted after $20 \mathrm{~h}$ of growth to solid agar plates and incubated for up to $24 \mathrm{~h}$ under asexual or sexual development inducing conditions.

A. nidulans strains for SILAC experiments were grown for $24 \mathrm{~h}$ in liquid media at $37^{\circ} \mathrm{C}$ under agitation. The medium for SILAC experiments was supplemented with $53.5 \mathrm{mM}$ isotopically heavy ${ }^{13} \mathrm{C}^{15} \mathrm{~N}$ labeled L-Lysine ${ }^{*} \mathrm{HCl}$ (SILANTES, München, Germany), isotopically medium D4 labeled L-lysine $2 \mathrm{HCl}$ 4,4,5,5-D4 (SILANTES) or unlabeled light L-lysine monohydrochloride (APPLIChEM, Darmstadt, Germany).

\subsubsection{Saccharomyces cerevisiae growth conditions}

Yeast strains were grown on yeast extract-peptone-dextrose growth medium (YPED) consisting of $2 \%(\mathrm{w} / \mathrm{v})$ bactopeptone, $1 \%(\mathrm{w} / \mathrm{v})$ yeast extract, $2 \%(\mathrm{w} / \mathrm{v})$ glucose. Liquid cultures were incubated on a rotary shaker at $30^{\circ} \mathrm{C}$ for up to 24 h. $2 \%(w / v)$ agar was added for solid plates. Agar plates were incubated for two to three days at $30^{\circ} \mathrm{C}$. After transformation of EGY48 yeast strain, yeasts were cultivated in Synthetic Complete (SC) medium containing 0,15\% (w/v) YNBaa-as (yeast nitrogen base w/o amino acids and ammonium sulfate), $0.5 \%$ (w/v) ammonium sulfate, $0.2 \mathrm{mM}$ inositol, $0.2 \%$ (w/v) amino acid powder mix and $2 \%(\mathrm{w} / \mathrm{v})$ raffinose. 


\subsection{Nucleic acid methods}

\subsubsection{Preparation of plasmid DNA from Escherichia coli}

E. coli strains carrying the desired plasmids were grown overnight $(\mathrm{o} / \mathrm{n})$ at $37^{\circ} \mathrm{C}$ under agitation in LB medium supplemented with ampicillin. Plasmid DNA was extracted with QIAprep ${ }^{\circledR}$ Spin Miniprep Kit (QIAGEN, Hilden, Germany) or the NucleoSpin ${ }^{\circledR}$ Plasmid Kit (MACHEREY-NAGEL, Düren, Germany) according to manufacturer's instructions. Elution of plasmid DNA from the

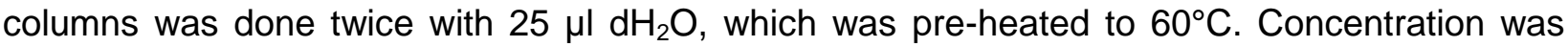
determined using NanoDrop ND-1000 photospectrometer. Long-term storage of DNA was done at $-20^{\circ} \mathrm{C}$.

\subsubsection{Isolation of $A$. nidulans genomic DNA}

A. nidulans cultures were grown $\mathrm{o} / \mathrm{n}$ at $37^{\circ} \mathrm{C}$ under agitation in flasks with indentations for isolation of genomic DNA (gDNA). Mycelia was harvested through Miracloth filters, washed with $0.96 \%(\mathrm{w} / \mathrm{v}) \mathrm{NaCl}$ and manually pestled in liquid nitrogen. $500 \mu \mathrm{g}$ gNA extraction buffer (200 mM Tris- $\mathrm{HCl}$ pH 8.5, 250 mM NaCl, 25 mM EDTA, $0.5 \%$ (w/v) SDS, recipe modified from Manian et al., 2001) were added to approximately $300 \mu \mathrm{l}$ grained mycelia and mixed properly through vortexing. Samples were incubated for at least $15 \mathrm{~min}$ at $65^{\circ} \mathrm{C}$ and subsequently cooled down on ice for $5 \mathrm{~min}$. $100 \mu \mathrm{l}$ of an $8 \mathrm{M}$ potassium acetate solution were added and carefully mixed with the mycelial solution by inversion. A 15 min centrifugation step at 13,000 rpm and room temperature (rt) leads to precipitation of proteins and cell debris. The precipitation step was repeated. The supernatant was transferred to a new reaction tube and mixed with $300 \mu \mathrm{l}$ isopropanol. After thoroughly inverting the mixture, samples were centrifuged for $15 \mathrm{~min}$ at $13,000 \mathrm{rpm}$ at rt. The pellet containing the gDNA was washed with $1 \mathrm{ml} 100 \%(\mathrm{v} / \mathrm{v})$ ethanol and the solution was centrifuged again for $5 \mathrm{~min}$. Finally, the DNA containing pellet was dried at $42^{\circ} \mathrm{C}$, dissolved in $100 \mu \mathrm{dH}_{2} \mathrm{O}$ and incubated at $37^{\circ} \mathrm{C}$ for one hour.

\subsubsection{Polymerase chain reaction (PCR)}

\subsubsection{Amplification of DNA fragments for cloning reactions}

DNA fragments were amplified with polymerase chain reactions (PCR) (Saiki et al., 1988). Template for PCR reactions was wild type genomic DNA from AGB551 if not indicated otherwise. DNA fragments for further cloning reactions were amplified with Phusion ${ }^{\circledR}$ High Fidelity Polymerase according to manufacturer's instructions (THERMO FISHER SCIENTIFIC) (Table 
1). This polymerase has proofreading activity and ensures high quality amplicons with very low mutation rate. Oligonucleotides used for PCR reactions are listed in Table 5. The annealing temperature of the primer pairs were calculated with the online OligoCalc tool (Kibbe, 2007). The $5 x$ high fidelity (HF) buffer as well as deoxynucleotide triphosphate mix (dNTPs) from THERMO FISHER SCIENTIFIC were used if not indicated otherwise.

\section{Table 1: PCR program used for Phusion High Fidelity polymerase.}

The annealing temperature varied depending on the oligonucleotides used for amplification. The extension steps were performed at $68^{\circ} \mathrm{C}$ instead of $72^{\circ} \mathrm{C}$ if the amplicon was longer than 4,000 bp. The final extension time was increased up to 15 min in these cases.

\begin{tabular}{|l|l|l|c|}
\hline Step & Temperature & Time & Cycle \\
\hline Initial denaturation & $98^{\circ} \mathrm{C}$ & $10 \mathrm{sec}$ & $1 \mathrm{x}$ \\
\hline Denaturation & $98^{\circ} \mathrm{C}$ & $30 \mathrm{sec}$ & \multirow{2}{*}{$34 \mathrm{x}$} \\
\hline Annealing & $55-65^{\circ} \mathrm{C}$ & $30 \mathrm{sec}$ & \\
\hline Extension & $72^{\circ} \mathrm{C} / 68^{\circ} \mathrm{C}$ & $30 \mathrm{sec} / \mathrm{kb}$ & $1 \mathrm{x}$ \\
\hline Final Extension & $72^{\circ} \mathrm{C} / 68^{\circ} \mathrm{C}$ & $10 \mathrm{~min} / 15 \mathrm{~min}$ & \\
\hline
\end{tabular}

\subsubsection{Colony PCR (cPCR)}

Colony PCRs (CPCR) were performed to verify that E. coli strains contain the desired DNA (Bergkessel and Guthrie, 2013). Taq Polymerase (THERMO FISHER SCIENTIFIC) deriving from Thermus aquaticus was used for cPCRs according to manufacturer's instructions (Table 2).

Table 2: PCR program used for Taq polymerase.

\begin{tabular}{|l|l|l|c|}
\hline Step & Temperature & Time & Cycle \\
\hline Initial denaturation & $95^{\circ} \mathrm{C}$ & $2 \mathrm{~min}$ & $1 \mathrm{x}$ \\
\hline Denaturation & $95^{\circ} \mathrm{C}$ & $30 \mathrm{sec}$ & \multirow{2}{*}{$29 \mathrm{x}$} \\
\hline Annealing & $55-65^{\circ} \mathrm{C}$ & $30 \mathrm{sec}$ & \\
\hline Extension & $72^{\circ} \mathrm{C}$ & $1 \mathrm{~min} / \mathrm{kb}$ & $1 \mathrm{x}$ \\
\hline Final Extension & $72^{\circ} \mathrm{C}$ & $10 \mathrm{~min}$ & \\
\hline
\end{tabular}

PCR master mix contained 10x Taq buffer $(200 \mathrm{mM}$ Tris $\mathrm{pH} 8.8,100 \mathrm{mM} \mathrm{KCl}, 100 \mathrm{mM}$ $\left(\mathrm{NH}_{4}\right)_{2} \mathrm{SO}_{4}, 22.5 \mathrm{mM} \mathrm{MgCl}_{2}{ }^{*} 6 \mathrm{H}_{2} \mathrm{O}, 0.02 \%$ (v/v) Nonidet P40 (NP40), $0.02 \%$ (v/v) TritonX-100, $40 \%$ (v/v) glycerol), dNTPs and respective oligonucleotides. A piece of single $E$. coli colonies were added into the reaction tubes and served as template. Colonies that carried the desired 
plasmids showed an amplicon and were used for isolation of the DNA for further verifications (test digestion, sequencing).

\subsubsection{Agarose gel electrophoresis}

DNA fragments were separated by size using agarose gel electrophoresis (Lee et al., 2012). $1 \%(\mathrm{w} / \mathrm{v})$ agarose was dissolved in TAE buffer (40 mM Tris, $20 \mathrm{mM}$ acetic acid, $1 \mathrm{mM}$ EDTA) and autoclaved for $5 \mathrm{~min}$ at $120^{\circ} \mathrm{C}$. Afterwards, $0.001 \mathrm{mg} / \mathrm{ml}$ ethidium bromide was added. The DNA was mixed with $6 x$ loading dye $(0.25 \%(\mathrm{w} / \mathrm{v})$ bromophenol blue, $0.25 \%(\mathrm{w} / \mathrm{v})$ xylene cyanole, $40 \%(\mathrm{w} / \mathrm{v})$ sucrose) prior to loading it on the agarose gel. The GeneRuler $1 \mathrm{~kb}$ DNA ladder (THERMO FISHER SCIENTIFIC) was used as size marker. Applying an electrical field to the running chamber lead to the separation of the DNA fragments by size, while negatively charged DNA molecules migrate to the positive electrode. TAE buffer was used as electrophoresis buffer. Visualisation of DNA fragments was done with UV light $(\lambda=254 \mathrm{~nm})$ in a Gel iX20 Imager Windows Version and the Intas GDS gel documentation software from INTAS SCIENCE IMAGING INSTRUMENTS GMBH (Göttingen, Germany) or on a TFX-20 MX Vilber Lourmat Super Bright transilluminator (SIGMA-ALDRICH).

\subsubsection{Purification of DNA from agarose gels}

DNA fragments separated by agarose gel electrophoresis were cut, extracted and purified from the agarose gel for further cloning reactions. Therefore, the QIAquick ${ }^{\circledR}$ Gel Extraction Kit from QIAGEN (Hilden, Germany) or the NucleoSpin ${ }^{\circledR}$ Gel and PCR Clean-up Kit from MACHEREYNAGEL GMBH \& CO. KG (Düren, Germany) were used according to manufacturer's instructions. Elution of DNA from the column was done twice by adding $20 \mu 60^{\circ} \mathrm{C}$ pre-heated $\mathrm{dH}_{2} \mathrm{O}$, respectively.

\subsubsection{Isolation of $A$. nidulans RNA and CDNA synthesis}

Total RNA was isolated from vegetative, asexual or sexually grown mycelia. Liquid cultures were inoculated using $1^{*} 10^{6}$ spores $/ \mathrm{ml}$ and grown for $20 \mathrm{~h}$ at $37^{\circ} \mathrm{C}$. Vegetative mycelial samples were harvested after this time, washed with $0.96 \%(\mathrm{w} / \mathrm{v}) \mathrm{NaCl}$ solution and immediately frozen in liquid nitrogen. Mycelia were shifted after $20 \mathrm{~h}$ of growth in liquid cultures on solid agar plates containing $30 \mathrm{ml} \mathrm{MM}$ and incubated for $24 \mathrm{~h}$ at $37^{\circ} \mathrm{C}$ under constant illumination to induce asexual development or at $37^{\circ} \mathrm{C}$ for $24 \mathrm{~h}$ in darkness and sealed with Parafilm to provoke 
oxygen limiting conditions to induce sexual development. Afterwards, mycelia were removed from the agar plate and immediately frozen in liquid nitrogen. Frozen mycelia of the different developmental stages were manually grained using pre-cooled mortar and pestle. Approximately $200 \mu \mathrm{l}$ of grained mycelia was used for RNA isolation. RNA extraction was performed with the RNeasy ${ }^{\circledR}$ Plant Miniprep Kit from QIAGEN (Hilden, Germany) according to manufacturer's instructions. Elution of RNA from the purification column was done twice with $15 \mu$ nuclease-free water (THERMO FISHER SCIENTIFIC), respectively. RNA concentrations were determined with a NanoDrop ND-1000 photospectrometer. For cDNA synthesis the QuantiTect ${ }^{\circledR}$ Reverse Transcription Kit from QIAGEN (Hilden, Germany) was used as recommended by the manufacturer using a two-step PCR protocol.

\subsubsection{Quantitative real-time polymerase chain reaction}

Quantitative real-time PCR (qRT-PCR) was performed using the MESA GREEN qPCR MasterMix Plus for SYBR ${ }^{\circledR}$ Assay purchased from EUROGENTEC (Lüttich, Belgium) to analyze gene expression levels. Oligonucleotides were mixed with nuclease free water and 1:10 diluted cDNA was mixed with light sensitive SYBR Green reagent. Primers and cDNA were combined in the FrameStar ${ }^{\circledR}$ 96-well plate purchased from 4TITUDE (Berlin, Germany). Prior to qRT-PCR, the plate was centrifuged for $2 \mathrm{~min}$ at $500 \mathrm{rpm}$ to remove air bubbles and ensure that all the reaction mix is at the bottom of the plate. The program used for qRT-PCR reactions is shown in Table 3 and respective oligonucleotides are listed in Table 4. Gene expression data were evaluated using the CFX Manager ${ }^{\mathrm{TM}} 3.1$ software package (BIORAD, München, Germany) using the 2- $\Delta \Delta \mathrm{C}_{\mathrm{T}}$ method for relative quantification (Schmittgen and Livak, 2008).

Table 3: qRT-PCR program used during this study.

\begin{tabular}{|l|l|l|c|}
\hline Step & Temperature & Time & Cycle \\
\hline 1 & $95^{\circ} \mathrm{C}$ & $2: 20 \mathrm{~min}$ & $1 \mathrm{x}$ \\
\hline 2 & $95^{\circ} \mathrm{C}$ & $20 \mathrm{sec}$ & \multirow{2}{*}{$39 \mathrm{x}$} \\
\hline 3 & $60^{\circ} \mathrm{C}$ & $22 \mathrm{sec}$ & \\
\hline 4 & $72^{\circ} \mathrm{C}$ & $22 \mathrm{sec}$ & $1 \mathrm{x}$ \\
\hline 5 & $95^{\circ} \mathrm{C}$ & $10 \mathrm{sec}$ & $1 \mathrm{x}$ \\
\hline 6 & Melt curve: $65^{\circ} \mathrm{C}$ to $95^{\circ} \mathrm{C}$, & $5 \mathrm{sec}$, respectively & \multicolumn{2}{c|}{} \\
\hline
\end{tabular}


The expression levels of $h 2 A$ (AN3468) and 15S rRNA served as reference for the relative quantification of gene expression levels. If not indicated otherwise, all qRT-PCR measurements were performed in at least two biological and three technical replicates, respectively.

Table 4: Primers used for qRT-PCR

Primer for qRT-PCRs were designed using the Primer3 software (Untergasser et al., 2012).

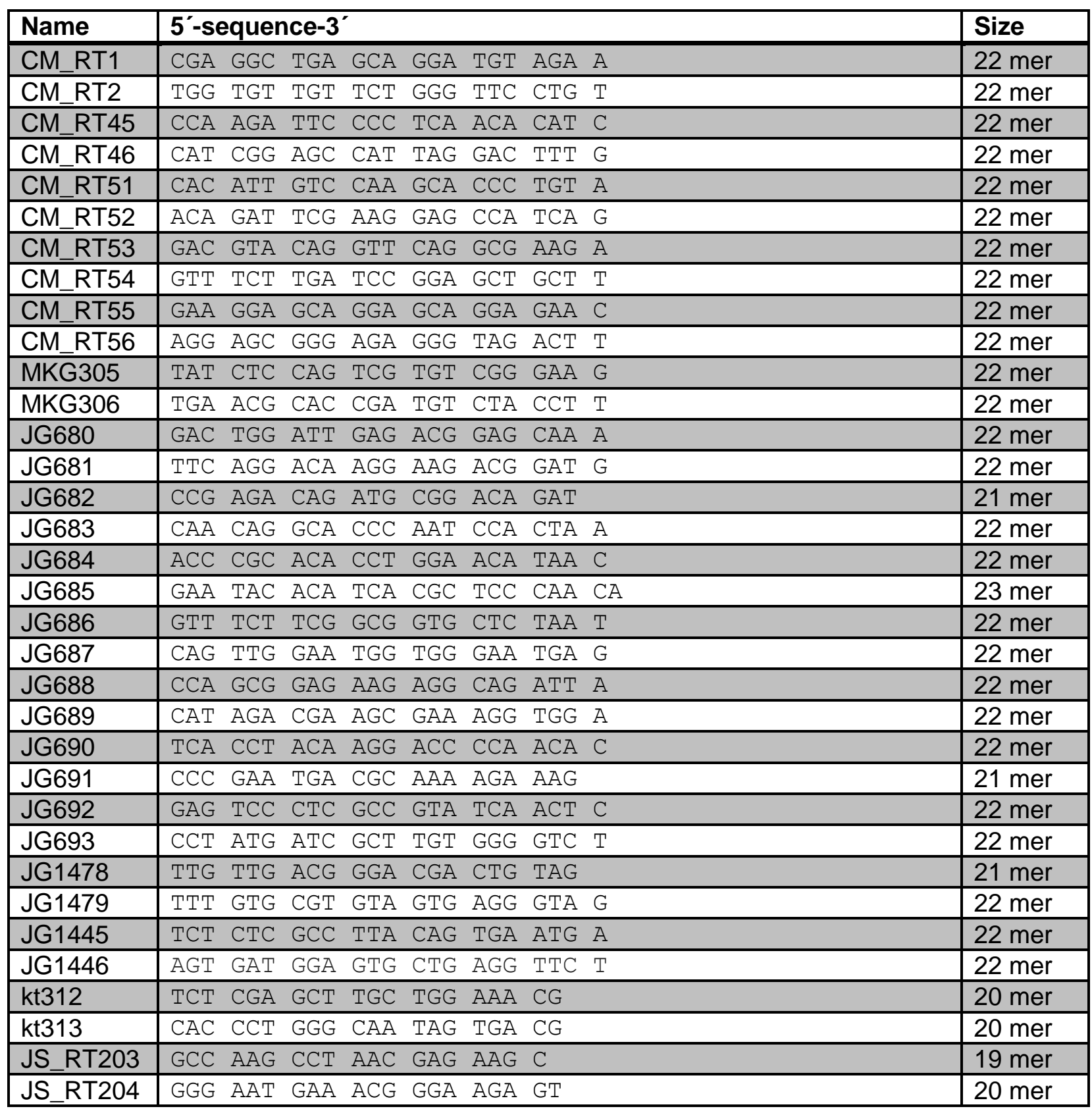




\subsection{Plasmid construction}

\subsubsection{Cloning strategies}

PCRs were used to amplify genes or surrounding flanking regions from genomic DNA (gDNA) or complementary DNA (cDNA), which were used for further cloning reactions. Fusion of different DNA fragments was performed prior to cloning reactions using fusion-PCR protocol established by Szewczyk and co-workers or during cloning reactions using the GeneArt® Seamless Cloning and Assembly Kit (THERMO FISHER SCIENTIFIC) or the GeneArt® Seamless Cloning and Assembly Enzyme Mix Kit (Thermo FISHER ScIENTIFIC) (Szewczyk et al., 2006). Long fusion PCR amplicons were pre-cloned into pJET Cloning Kit (THERMO FISHER SCIENTIFIC) according to manufacturer's instructions. As cloning vectors the pUC19L vector provided with the GeneArt尺 Kits or pBluescript SK+ were used. The outermost primers of each construct generated in this study contain additionally a Pmel (Mssl) cutting site (GTTT^AAAC).

\subsubsection{Seamless Cloning reaction}

Primers used for Seamless Cloning reactions always encompassed in addition to the Pmel (Mssl) cutting site a $15 \mathrm{bp}$ long overhang to the neighboring fragment. Cloning reaction was performed as given in manufacturer's instructions (THERMO FISHER SCIENTIFIC). After mixing of the DNA fragments with the linearized pUC19L vector, the buffer and the enzyme, the reaction was incubated for $30 \mathrm{~min}$ at rt, cooled down for $5 \mathrm{~min}$ on ice and transformed into E. coli $\mathrm{DH} 5 \mathrm{a}$. Up to three different DNA fragments in addition to the vector backbone could be used for cloning reactions with the GeneArtß Seamless Cloning and Assembly Enzyme Mix Kit.

\subsubsection{Ligation}

For the cloning of only one DNA fragment regular ligation reactions were performed. The DNA:vector ratio was 5:1. Ligation reactions were performed in presence of $10 \%(\mathrm{v} / \mathrm{v})$ PEG4000 and the T4 DNA Ligase (THERMO FISHER SCIENTIFIC) was used. The ligation mixture was incubated overnight at $16^{\circ} \mathrm{C}$. DNA fragments were phosphorylated and the vector backbone was dephosphorylated prior to the ligation reaction. Therefore, the fragment was incubated with Buffer A (PNK), ATP and the T4 PNK enzyme (THERMO FISHER SCIENTIFIC); dephosphorylation of the vector was done using the FastAP enzyme (THERMO FISHER SCIENTIFIC) according to manufacturer's instructions. The whole ligation reaction was used for transformation into $E$. coli. 


\subsubsection{Recycable marker cassette}

If it is not indicated otherwise, plasmids generated during this study contain a recyclable marker resistance cassette. Phleomycin resistance resulting from the expression of the ble gene deriving from Streptoalloteichus hindustanus or the nourseothricin (clonNAT) resistance deriving from the expression of the nat1 gene deriving from Streptomyces noursei were used respectively (Drocourt et al., 1990; Kück and Hoff, 2006).

The recyclable cassette consists of two six sites, which border the gene encoding the $\beta$-recombinase $(\beta-r e c)$ that is under the control of a xylose-inducible promotor $\left({ }^{\mathrm{P}} x y\right)$ and terminated by the trp $C^{\top}$ terminator (Hartmann et al., 2010). Furthermore, a constitutively active gpdA promotor $\left({ }^{\mathrm{P} g p d A}\right)$ controls the expression of the gene conferring the resistance (phleo; clonNAT) (Figure 9).

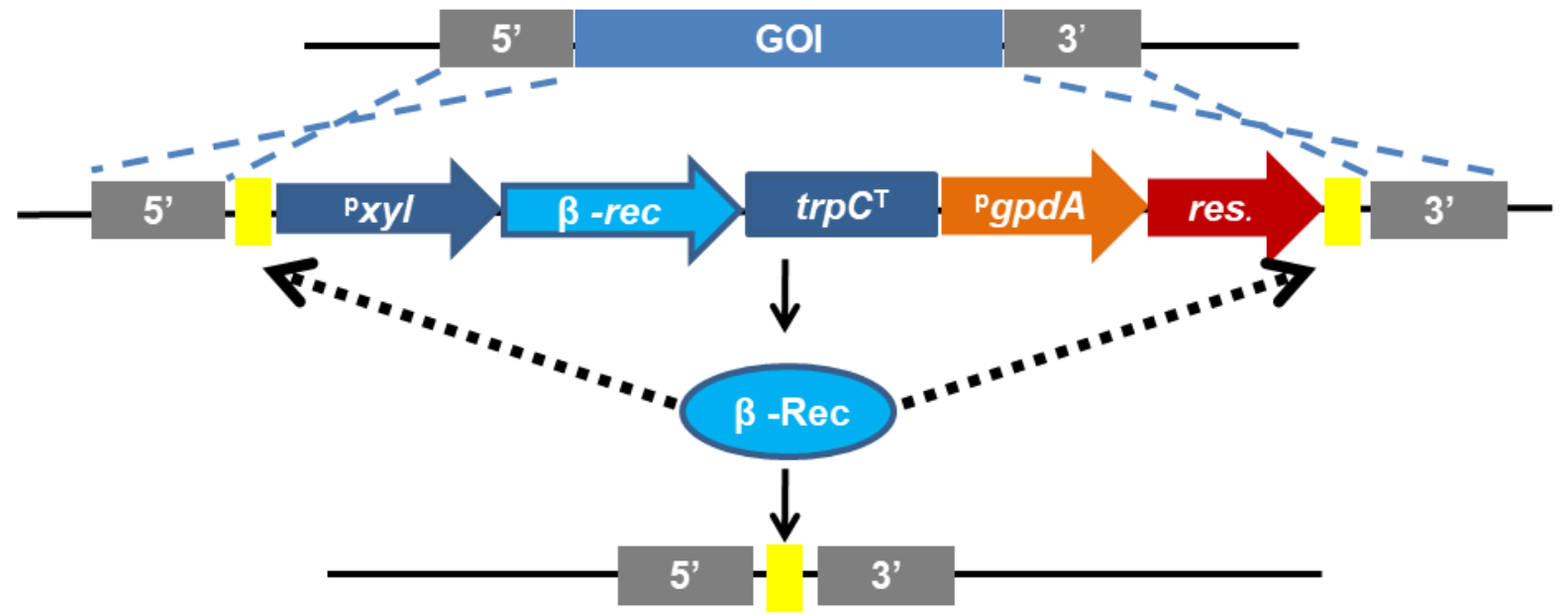

Figure 9: Schematic representation of the recyclable marker system.

The gene of interest (GOI) can be exchanged with a recyclable marker cassette by homologous recombination. The cassette is surrounded by two six sites (depicted in yellow) and consists of a gene encoding the $\beta$-recombinase $(\beta-r e c)$, which is under the control of a xylose inducible promotor $\left({ }^{\mathrm{P}} x y l\right)$ and a trpC terminator $\left(\operatorname{trp} C^{\mathrm{T}}\right)$. The resistance marker (res; phleomycin or clonNAT) is under the control of the constitutively active gpdA promotor ( $\left.{ }^{\mathrm{P} g p d A}\right)$. Xylose induces the expression of the $\beta$-recombinase, which excises the whole cassette out of the genome.

Successful transformation and verification of the construct containing this marker cassette is followed by the inoculation of $A$. nidulans strains on MM containing $0.5 \%(w / v)$ xylose and $0.5 \%$ $(w / v)$ glucose. This induces the transcription of the $\beta$-recombinase encoding gene, which product excises the marker cassette at the six sites. One six site is left after marker recycling (Figure 9). 


\subsubsection{Sequencing of plasmids or PCR products}

PCR products or plasmids generated in this study were sequenced by SEQLAB SEQUENCE LABORATORIES GMBH (Göttingen, Germany). The received sequences were analyzed with the Lasergene software package (DNA STAR INC.).

\subsubsection{Plasmid and strain construction}

gDNA of AGB551 was used as template for PCR reactions if not indicated otherwise. All given fragment sizes are rounded in the following chapter. All generated plasmids were confirmed with test digestions and sequencing. The outermost primers of the constructs generated in this study contained in their overhangs Pmel cutting sites if not stated otherwise. The used primers are listed in Table 5, the constructed plasmids in Table 6. Locus information of single genes was obtained from AspGD, Cadre or FungiDB (Cerqueira et al., 2014; Mabey Gilsenan et al., 2012; Stajich et al., 2012). The correct integration of the transformed DNA fragments was verified with Southern hybridization experiments.

Table 5: Primers used in this study for amplification, sequencing and cloning of plasmids.

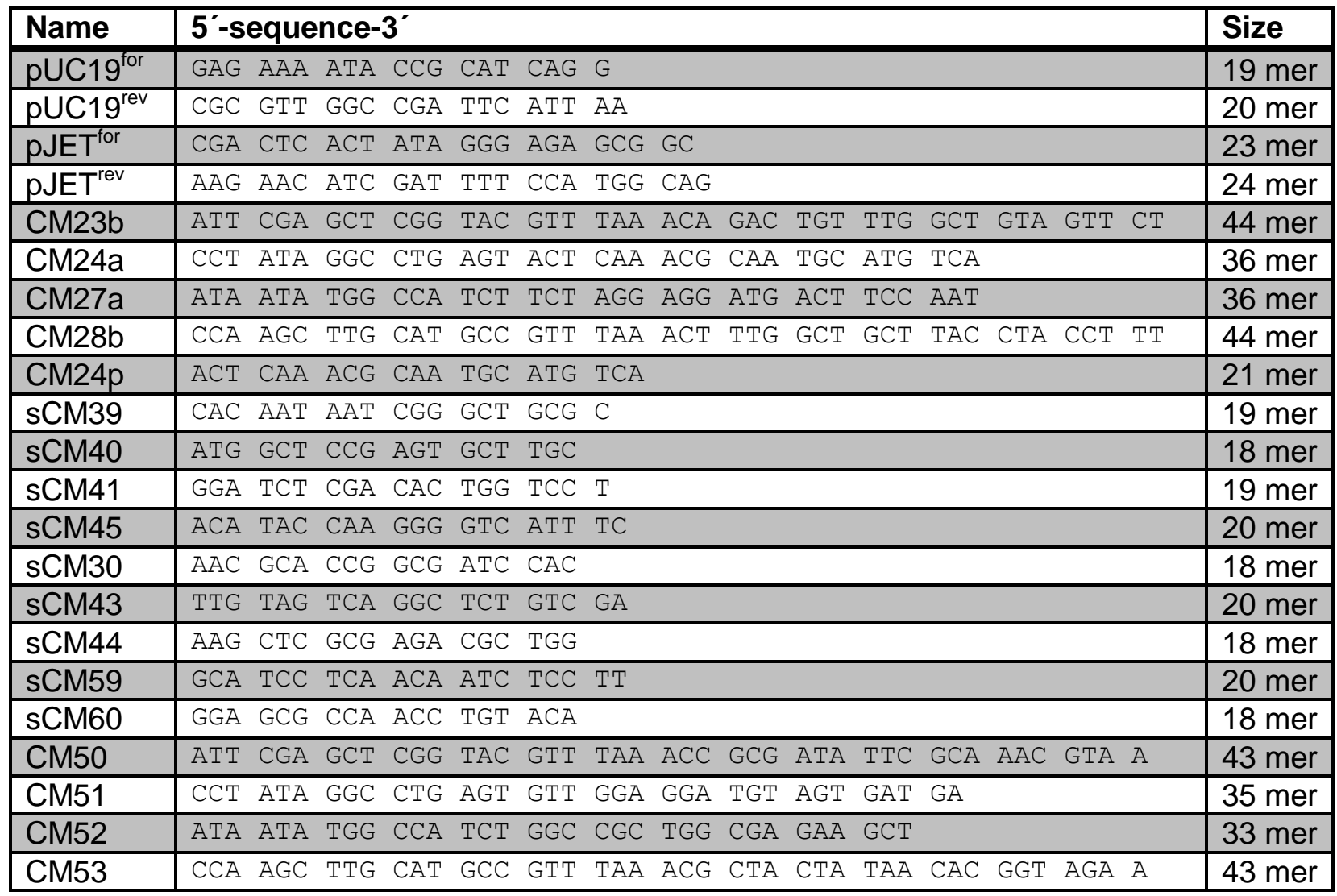


Table 5: continued.

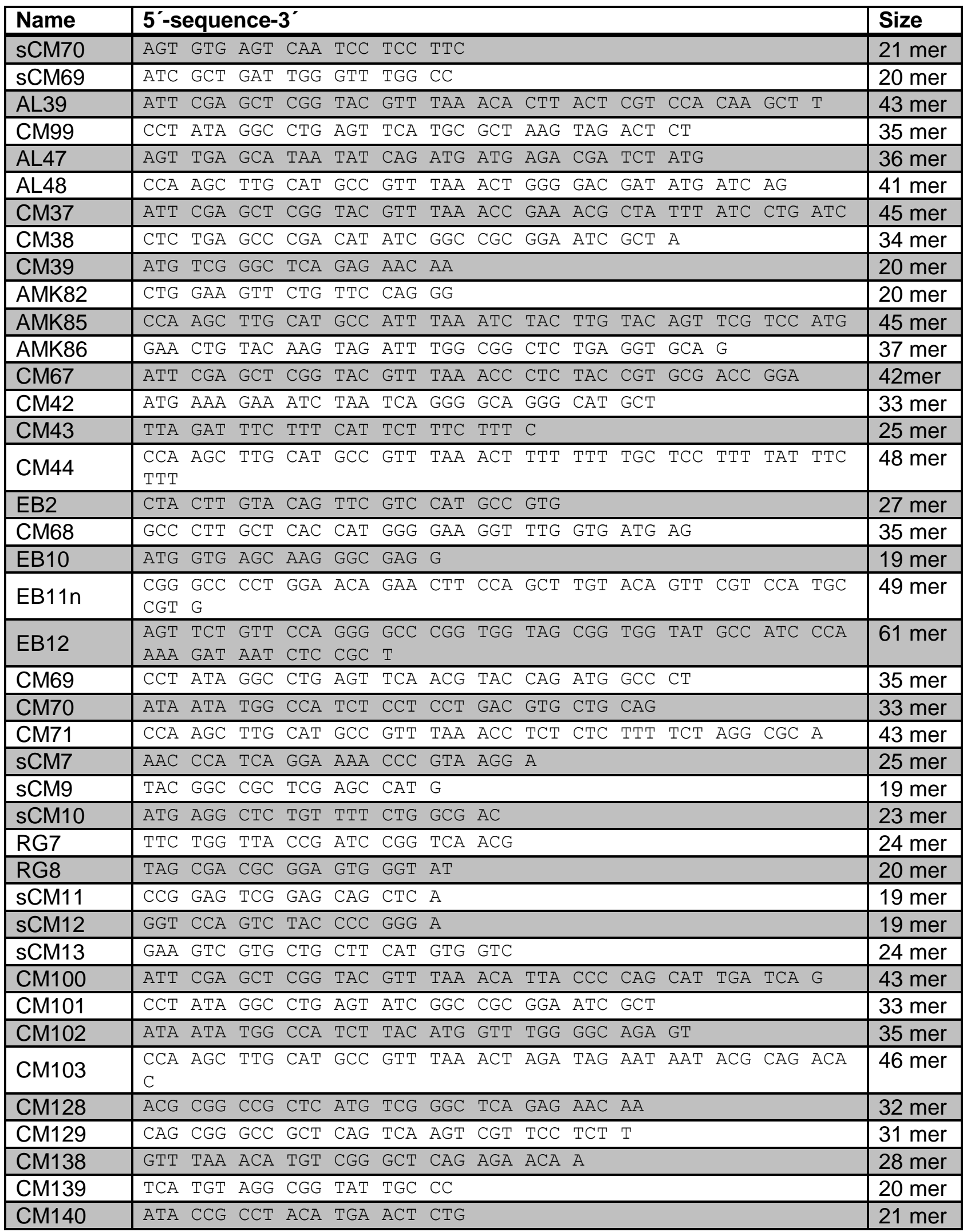


Table 5: continued.

\begin{tabular}{|c|c|c|}
\hline Name & $5^{\prime}$-sequence-3' & Size \\
\hline CM141 & TCT TTG GCG CGC GGA CAA TA & 20 mer \\
\hline CM142 & GCG CGC CAA AGA GCA TCG A & 19 mer \\
\hline kt183 & GAT GTG CTG CAA GGC GAT TAA GTT G & 25 mer \\
\hline kt184 & GGC TTT ACA CTT TAT GCT TCC G & 22 mer \\
\hline flip-1 & ACC TAT AGG CCT GAG ATT TAA ATA TCG AAT TCC TGA AGC CCG G & 43 mer \\
\hline flip-2 & ATA ATA TGG CCA TCT CAC GTG ATC AAG CTT ATC GAT ACC GTC G & 43 mer \\
\hline CM2 & TCA GTC AAG TCG TTC CTC TTC ACC C & $25 \mathrm{mer}$ \\
\hline CM161 & GCC ACG GGC GCG CCG TCG GGC TCA GAG AAC AAG C & $34 \mathrm{mer}$ \\
\hline CM162 & TCA CAT GAT ATA GAC GTT GTG GC & 23 mer \\
\hline CM163 & CGC TCC ATC GCC ACG TCA GAC GAC GAT GAT TTC ATG C & 37 mer \\
\hline CM164 & TTA GAA CAG GCC CGT CTT CA & 20 mer \\
\hline CM165 & TCA CTT GTA CAG CTC GTC CA & 20 mer \\
\hline CM166 & CGC TCC ATC GCC ACG CCA GAC GAA GCC ATA TCC AT & $35 \mathrm{mer}$ \\
\hline CM167 & TTA TCC GAG TGC CAC GCC A & 19 mer \\
\hline CM94 & CGT GGC GAT GGA GCG CAT & 18 mer \\
\hline AMK163 & ATG GTG AGC AAG GGC GAG & 18 mer \\
\hline AMK168b & ATG GCC GAC AAG CAG AAG AAC G & 22 mer \\
\hline AMK169b & CGG GCG GCC CGT GGC GAT GGA GCG CTT GTA CAG CTC GTC CAT GC & 44 mer \\
\hline SR171 & ACC TTG ATC TGG CAT ATC TAC CA & 23 mer \\
\hline SR172 & ACG GCC GTG TAC ATA TCA TGT C & 22 mer \\
\hline SR173 & CGT TGT GCC ACA CTA TGG ATT C & 22 mer \\
\hline SR174 & TAG GTA TAA TGC ATA GAA CCG CC & 23 mer \\
\hline CM171 & AG GAA TTC GAT ATT TGT TTA AAC GCA CCA TCT TGC GCC GAA & $41 \mathrm{mer}$ \\
\hline CM172 & TTC GCC CTT GCT GAC CATT ATG TGC GGC AGT CTT GAG T & 38 mer \\
\hline CM173 & ATG GTC AGC AAG GGC GAA GA & 20 mer \\
\hline CM174 & $\begin{array}{lllllllllll}\text { TCA } & \text { TCG TCG TCT GAC ATA CCA CCG CTA CCA CCC TTG TAC AGC TCG } \\
\text { TCC ATG } & \text { C }\end{array}$ & 52 mer \\
\hline CM175 & ATGTCAGACGACGATGATTTCA & 22 mer \\
\hline CM176 & ATA GGC CTG AGA TTT TTA GAA CAG GCC CGT CTT CAT & 36 mer \\
\hline CM177 & ATA TGG CCA TCT CAC GCG GGG GAG GGG GAG G & $31 \mathrm{mer}$ \\
\hline CM178 & GAT AAG CTT GAT CAC GTT TAA ACA CAC TCC ACA GCC CAC GAA G & 43 mer \\
\hline
\end{tabular}

Table 6: Plasmids used in this study.

${ }^{\mathrm{P}}=$ promotor ${ }^{\mathrm{T}}=$ terminator, af $=$ Aspergillus fumigatus, p.c. $=$ personal communication, ${ }^{\mathrm{C}}=\mathrm{C}$ terminal, ${ }^{\mathrm{N}}=\mathrm{N}$-terminal, $\mathrm{RM}=$ recyclable marker cassette

\begin{tabular}{|c|c|c|}
\hline Plasmid & Description & Reference \\
\hline pUC19L & cloning vector, $a m p^{R}$ & Thermo Fisher Scientific \\
\hline pBluescript KS & cloning vector, $a m p^{R}$ & Fermentas $\mathrm{GmbH}$ \\
\hline pME4313 & 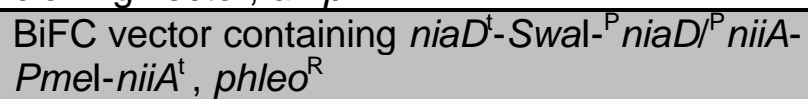 & J. Gerke, p.c. \\
\hline pME4319 & six- ${ }^{\mathrm{P}} x y l P:: \beta-r e c:: \operatorname{trp}^{t}-$ phleo $^{\mathrm{R}}-$ six & J. Gerke, p.c. \\
\hline pME4304 & six $^{-}{ }^{\mathrm{P}} x y \mid P:: \beta-$ rec:: $\operatorname{trp} C^{t}-g p d A^{P}: n a t^{R}-\operatorname{six}$ & J. Gerke, p.c. \\
\hline pME3857 & ${ }^{\mathrm{P}}$ gpdA::mrfp::h2A::hisB ${ }^{\dagger} ;$ phleo ${ }^{\mathrm{R}}$ in pBluell SK+ & (Bayram et al., 2012) \\
\hline
\end{tabular}


Table 6: continued.

\begin{tabular}{|c|c|c|}
\hline Plasmid & Description & Reference \\
\hline pME3281 & $\begin{array}{l}\text { phleo cassette; shortened }{ }^{P} g p d A:: b l e:: t r p C^{\top}, \\
\text { shortened phleo cassette blunted into pBluescript, } \\
\text { bla }\end{array}$ & $\begin{array}{l}\text { (Helmstaedt et al., } \\
\text { 2011) }\end{array}$ \\
\hline pME3173 & ${ }^{P}$ gpdA::mrfp::h2A::hisB ${ }^{T}:$ nat $^{R}$ & (Bayram et al., 2008b) \\
\hline pAMK129 & PgpdA::mcherry::fbx15 1 NLS1 & A.M.Köhler, p.c. \\
\hline pME4579 & PztfA::sgfp::ztfA::phleoRM & K. Thieme, p.c. \\
\hline pME4652 & candA-C::gfp & A.M.Köhler, p.c. \\
\hline pME4662 & $\begin{array}{l}{ }^{\top} \text { niiA:::yfp }{ }^{N .: P} \text { niiA } / \text { niaD }{ }^{P}:: y f p^{C}:: \text { cand }-N:: \text { niiD }^{\top}:: \text { phleo } \\
\text { in pME4313, bla }\end{array}$ & A.M.Köhler, p.c. \\
\hline pME4696 & $\begin{array}{l}\text { cloning vector containing Pmll restriction site:::six- } \\
{ }^{{ }_{x}} \text { xyIP:: } \beta-\text {-rec::trp } C^{t}-\text { nat }^{R} \text {-six ::Swal restriction site }\end{array}$ & this study \\
\hline pME4697 & $5^{\text {,ysA }}$-IysA::natRM-3, ${ }^{\text {,ysA }}$ & this study \\
\hline pME4698 & $5^{, / y S A}-1 y S A-3^{, / y S A}$ & this study \\
\hline pME4699 & $5^{\operatorname{argc}}-\arg C::^{\prime}$ atRM-3, ${ }^{\operatorname{argc} C}$ & this study \\
\hline pME4700 & $5^{\prime a r g C}-\arg C-3^{\prime a r g C}$ & this study \\
\hline pME4654 & $5^{\text {CSNE }}-$ phleoRM -3, CSNE & A.M.Köhler, p.c. \\
\hline pME4701 & $5^{\prime C S n E}-\operatorname{csnE}:: n a t R M-3^{\prime c S n E}$ & this study \\
\hline pME4702 & $5^{\operatorname{csn} D}-$ gfp:: $\operatorname{csnD}:: n a t R M-3^{\prime c s n D}$ & this study \\
\hline pME4703 & $5^{\text {uspA }}-$ pyro $A^{A f}-3^{\text {,uspA }}$ & J. Schinke, p.c. \\
\hline pME4704 & $5^{\text {uspA }}-$ uspA $-3^{\prime \text { uspA }}$ in pME3281 & J. Schinke, p.c. \\
\hline pME4705 & $5^{\prime u s p A}-$ natRM $-3^{\prime \text { uspA }}$ & this study \\
\hline pME4706 & $5^{\text {uspa }}-$ uspA::gfp::P gpdA::nat $-3^{\text {,uspa }}$ & this study \\
\hline pME4707 & $5^{\prime u s p A}-$ usp $A^{A A}:: g f p:: n a t R M-3^{\prime u s p A}$ & this study \\
\hline pME4708 & $\begin{array}{l}{ }^{P} \text { niaD::ceyfp:: uspA::niaD in Swal site, }{ }^{\top} \text { niiA:: } \\
\text { neyfp::csnB::niiA }{ }^{\top} \text { in Pmel site of pME4313 }\end{array}$ & this study \\
\hline pME4709 & $\begin{array}{l}{ }^{\mathrm{P}} \text { niaD::ceyfp::niaD }{ }^{\top} \text { in Swal site, }{ }^{\mathrm{P}} \text { niiA:: } \\
\text { neyfp::csnB::niiA }{ }^{\top} \text { in Pmel site of pME4313 }\end{array}$ & this study \\
\hline pME4710 & $\begin{array}{l}{ }^{\mathrm{P}} \text { niaD:: ceyfp:: } \text { niaD }^{\top} \text { in Swal site, }{ }^{\mathrm{P}} \text { niiA:: } \text { neyfp:: } \\
\text { nii }^{\top} \text { in Pmel site of pME4313 }\end{array}$ & this study \\
\hline pME4711 & $\begin{array}{l}{ }^{\mathrm{P}} \text { niaD::ceyfp::UspA::niaD }{ }^{\top} \text { in Swal site, }{ }^{\mathrm{P}} \text { niiA:: } \\
\text { neyfp::csnF::niiA }{ }^{\top} \text { in Pmel site of pME4313 }\end{array}$ & this study \\
\hline pME4712 & $\begin{array}{l}{ }^{P} \text { niaD::ceyfp::niaD }{ }^{\top} \text { in Swal site, }{ }^{P} \text { niiA:: } \\
\text { neyfp::csnF::niiA }{ }^{\top} \text { in Pmel site of pME4313 }\end{array}$ & this study \\
\hline pME4713 & $5^{\prime c s n B}$ - mcherry::csnB::phleoRM-3'uspA & this study \\
\hline pME4714 & $5^{\text {veA }}-v e A:: g f p:: n a t R M-3^{\prime v e A}$ & S. Thieme, p.c. \\
\hline pME4687 & $5^{\text {velB}}$ - velB::gfp::natRM-3'velB & S. Thieme, p.c. \\
\hline pME3230 & $\begin{array}{l}\text { bla, }{ }^{P} \text { GAL1::B42::MCS:: } A D H^{T}, T R P 1,2 \mathrm{~mm} / \text { two } \\
\text { hybrid prey vector }\end{array}$ & (Busch et al., 2007) \\
\hline pME3229 & $\begin{array}{l}\text { bla, }{ }^{P} A D H:: l e x A:: M C S:: A D H^{T}, H I S 1,2 m m / t w o \\
\text { hybrid bait vector }\end{array}$ & (Busch et al., 2007) \\
\hline pME4715 & $\begin{array}{l}\text { bla, }{ }^{P} A D H:: l e x A:: \text { UspA }^{c D N A}:: A D H^{T}, H I S 1,2 \mathrm{~mm} / \\
\text { two hybrid bait vector in Notl restriction site }\end{array}$ & this study \\
\hline pME2502 & csnA cDNA of pME2987 (Xhol) in pEG202 & (Busch et al., 2007) \\
\hline pME2978 & csnB cDNA of pME2988 (Xhol) in pJG4-5 & (Busch et al., 2007) \\
\hline pME2979 & csnC cDNA of pME2989 (Xhol) in pJG4-5 & (Busch et al., 2007) \\
\hline
\end{tabular}


Table 6: continued.

\begin{tabular}{|l|l|l|}
\hline Plasmid & Description & Reference \\
\hline pME2355 & csnD cDNA of pME2990 (Xhol) in pJG4-5 & (Busch et al., 2007) \\
\hline pME2980 & csnE cDNA of pME2991 (EcoRl) in pJG4-5 & (Busch et al., 2007) \\
\hline pME2981 & csnF cDNA of pME2992 (EcoRI) in pJG4-5 & (Busch et al., 2007) \\
\hline pME2982 & csnG cDNA of pME2993 (EcoRl) in pJG4-5 & (Busch et al., 2007) \\
\hline pME2983 & csnH cDNA of pME2987 (EcoRI) in pJG4-5 & (Busch et al., 2007) \\
\hline pME2501 & csnA cDNA of pME2987 (Xhol) in pEG202 & (Busch et al., 2007) \\
\hline pME2978 & csnB cDNA of pME2988 (Xhol) in pJG4-5 & (Busch et al., 2007) \\
\hline
\end{tabular}

\subsubsection{Construction of the cloning vector pME4696}

The vector pBluescript KS was amplified with primers flip-1 and flip-2, which contained a Swall or Pml restriction site in their overhang. The linear PCR fragment and the Sfil digested nourseothricin recyclable marker cassette (natRM) from plasmid pME4304 were ligated in a Seamless cloning reaction. The resulting plasmid was named pME4696 and served as cloning vector for further constructs.

\subsubsection{Construction of the $\Delta / y s A$ deletion cassette and $A$. nidulans strain}

For construction of the $\Delta l y s A$ deletion cassette the 1,100 bp long 5' flanking region was amplified using primer pair CM23b/CM24a. Primer CM23b contains in its overhang a Pmel cutting site and a $15 \mathrm{bp}$ complementary region to $\mathrm{pUC19L}$ vector. Primer CM24a includes a $15 \mathrm{bp}$ overhang to the six site of the natRM. The 1,600 bp 3' flanking region was amplified with the primer pair CM27a/CM28b, whereas CM27b contains 15 bp overhang to the six site of the recyclable marker cassette and CM28b contains an extension with a Pmel cutting site and 15 bp overhang to the plasmid backbone of pUC19L. The 15 bp overhang is necessary for further fusion of the fragments using the GeneArt ${ }^{\circledR}$ Seamless Cloning and Assembly Kit according to manufacturer's instructions. The resulting plasmid is pME4697 and was transformed into AGB552 resulting in strain AGB1092.

\subsubsection{Construction of the IysA complementation plasmid and $A$. nidulans strain}

The construction of the lysA complementation plasmid was performed by amplifying the whole gene locus of AN2873 including 5' and 3' flanking regions with primers CM23b and CM28b encompassing 4,500 bp. The resulting construct (pME4698) was cut with Pmel and transformed as linear plasmid in AGB1092 resulting in strain AGB1093. 


\subsubsection{Plasmid and strain construction of gfp::csnD}

The 5' flanking region of $c s n D$ was amplified with primer pair CM67/CM68 and encompasses $700 \mathrm{bp}$, whereas CM68 has a $15 \mathrm{bp}$ overhang to the neighboring gfp. gfp was amplified from pME4579 with primer pairs EB10/EB11n. csnD ORF was amplified from gDNA with primers EB12/CM69. Primer EB11n and EB12 contain long overhangs encompassing the PreScission Protease cleavage site and a GGSGG encoding linker sequence. gfp and csnD ORF were combined using fusion-PCR resulting in a 2,000 bp fragment. Primer CM69 contained a 15 bp overhang to the six site of the recyclable marker cassette. The $700 \mathrm{bp}$ encompassing 3' flanking region was amplified with $\mathrm{CM} 70 / \mathrm{CM} 71$. The single fragments were fused through a Seamless Cloning reaction and resulted in plasmid pME4702. The construct was excised with Pmel and transformed into AGB1092 resulting in strain AGB1151.

\subsubsection{Construction of the $\Delta / y s A, g f p:: \operatorname{csn} D, \Delta c s n E$ SILAC strain}

For generation of csnE deletion in AGB1151, pME4654 was digested with Pmel. The resulting linear csnE deletion cassette was transformed into AGB1151 and resulted after marker recycling in AGB1152.

\subsubsection{Construction of the csnE complementation plasmid and $\boldsymbol{A}$. nidulans strain}

For the csnE complementation plasmid the 5' flanking region together with the csnE ORF was amplified with primer pair AL39/CM99 and encompassed 1,900 bp. The 1,000 bp long 3' flanking region was amplified with AL47/AL48. All fragments were fused together in a Seamless Cloning reaction. Therefore, all primers contained 15 bp overhangs complementary to the neighboring fragment to allow fusion of the fragments. The resulting plasmid is pME4701. Excising of the complementation construct with Pmel and transformation of the linear fragment into AGB1152 resulted in the strain AGB1153.

\subsubsection{Construction of the $\Delta \arg C$ deletion cassette and $A$. nidulans strain}

The $\arg C$ gene (AN1883) was deleted to generate an arginine auxotrophic $A$. nidulans strain. The 1,000 bp encompassing 5' flanking region was amplified with the primer pair CM50/CM51. CM50 has a 15 bp overhang to the pUC19 plasmid backbone, whereas CM51 has a primer overhang towards the six site of the natRM. The 3' flanking region has a length of 1,100 bp and was amplified with primer pair CM52/CM53 containing respective overhangs necessary for the 
following Seamless Cloning reaction. The resulting plasmid is pME4699. The deletion cassette was excised with Pmel and the linear DNA fragment transformed into AGB552 resulting in AGB1154.

\subsubsection{Construction of $\arg C$ complementation plasmid and $A$. nidulans strain}

For complementation of the arginine auxotrophic strain AGB1154, the $\arg C$ gene itself served as marker. Therefore, the 4,000 bp encompassing genomic locus including 5' flanking region, arg $C$ ORF and 3' flanking region was amplified with CM50 and CM53 and cloned into pUC19 vector in a Seamless Cloning reaction. The resulting plasmid was named pME4700. The complementation construct was cut out with Pmel and the linear DNA fragment was transformed into AGB1154, resulting in AGB1155.

\subsubsection{Construction of the $\Delta u s p A^{p y r o A}$ and complementation comp pyroA $A$. nidulans strain}

The plasmid pME4703 was used to generate a deletion construct of uspA (AN6354), while replacing the uspA ORF with the pyroA marker derived from Aspergillus fumigatus. Therefore, the deletion cassette was excised from pME4703 in a double digest reaction using the restriction enzymes BamHI and Xhol and the linear DNA fragment was transformed into AGB551 resulting in the strain AGB1156.

Complementation was done by transforming the circular plasmid pME4704 into AGB1156. Through ectopic integration of the UspA ORF into the deletion strain carrying the $p y r o A^{A f}$ marker at the original uspA gene locus, strain AGB1157 was generated.

\subsubsection{Construction of the $\Delta u s p A^{S i x}$ plasmid and A. nidulans strain}

The 5' flanking region encompassing 1,800 bp was amplified with primer pair CM100/CM101 containing overhangs to the pUC19 plasmid backbone or the six site of the natRM cassette. The 1,800 bp encompassing 3' flanking region was amplified with the primer pair CM102/CM103. All fragments were ligated in a Seamless Cloning reaction and the resulting plasmid was named pME4705. The deletion cassette was excised from pME4705 with Pmel and transformed as linear fragment into AGB551 resulting in AGB1158. 


\subsubsection{Construction of the uspA::gfp fusion plasmid and $A$. nidulans strain}

The uspA::gfp containing plasmid was cloned in two steps. The 5' flanking region together with the uspA ORF was amplified using primers CM37/CM48. The PreScission protease cleavage site, the GGSGG linker encoding sequence and the gfp was amplified from pME4652 using primer AMK82 and AMK85. AMK85 contained a Swal cutting site in its primer overhang. Both fragments were cloned into pUC19 in a Seamless Cloning reaction. The resulting pre-plasmid was linearized with Swal and used as backbone for the second Seamless Cloning reaction Therefore, the ${ }^{\mathrm{P}} g p d A$ together with the nat resistance gene was amplified from pME4304 with primers AMK86/CM42, which encompassed 1,400 bp. The 900 bp long uspA 3' flanking region was amplified with the primer pair CM43/CM44. Both fragments were cloned into the Swal cutting site of the pre-plasmid in another Seamless Cloning reaction. The resulting plasmid was named pME4706 and excised with Pmel to transform the linear construct into AGB551 resulting in AGB1159. The plasmid pME4706 was also transformed into AGB1158 and served as complementation strain resulting in AGB1160. The plasmid pME3857 was transformed ectopically into AGB1159 resulting in strain AGB1161. This strain was used to visualize nuclei in fluorescence microscopy.

\subsubsection{Construction of the $u s p A^{A A}:: g f p$ fusion plasmid and $A$. nidulans strain}

For the construction of an inactive uspA mutant, the uspA ORF was amplified in three different parts. The first part of the ORF was amplified with primer pair CM138/CM139 and encompassed 1,400 bp. The cysteine at position 469 (TGC) was exchanged to an alanine (GCC) by mutations introduced with the primers CM139 and CM140 at the corresponding position. The second part of the ORF was $1,800 \mathrm{bp}$ long and amplified with CM140/CM141. The primer CM141 and CM142 contain the mutation of the triplet encoding the cysteine (TGC) at position 1,066 to an alanine (GCC). The CM142/EB2 primer pair was used to amplify the last 1,900 bp long fragment consisting of the part of the uspA ORF together with the gfp. For these PCRs the plasmid pME4706 served as template. All three parts were fused together in single fusion-PCR reactions and cloned into the Pml cutting site of pME4696. The 3' flanking region of uspA encompassing 900 bp was amplified with primers CM43/CM44 and included into the Swal site of pME4696 through a classical ligation reaction. The resulting plasmid is pME4707. The construct was excised with Pmel restriction enzyme and transformed into AGB551 resulting in AGB1162. The presence of the point mutations was additionally verified by sequencing the genomic DNA of 
strain AGB1162 at corresponding positions. The plasmid pME3857 was transformed into AGB1162 and resulted in strain AGB1163.

\subsubsection{Construction of the VeA-GFP expressing $A$. nidulans strains}

The fragment used for transformation was excised from plasmid pME4714 with Pmel and the resulting linear DNA fragment was transformed into AGB1156 resulting in the strain AGB1164.

\subsubsection{Generation of strains expressing VeA-GFP and RFP tagged histones}

AGB1165 and AGB1164 were transformed with the circular plasmid pME3857 to visualize nuclei due to RFP-tagged histones during fluorescence microscopy experiments. Transformation of AGB1164 with pME3857 resulted in AGB1166; transformation of AGB1165 with pME3857 resulted in strain $A G B 1167$.

\subsubsection{Strain construction of VelB-GFP expressing $\boldsymbol{A}$. nidulans strains}

The velB::gfp construct was excised out from pME4687 using Pmel restriction enzyme and the linear DNA fragment was transformed into AGB1156 resulting in the strain AGB1168.

\subsubsection{Construction of $\Delta c s n E$ strain in A. nidulans AGB551 strain}

The csnE deletion cassette was excised from plasmid pME4654 with Pmel and transformed into AGB551 resulting in strain AGB1169.

\subsubsection{BiFC plasmid and strain construction of UspA and CsnB in A. nidulans}

For BiFC experiments, the plasmid pME4313 was used as backbone, which contained the bidirectional nitrate promotor. The C-terminal half of $y f p$ (cyfp) was fused N-terminally to uspA and ligated into the Swal cutting site of pME4313. cyfp together with the linker was amplified with primers AMK168b/AMK169b from plasmid pME4662. The resulting $300 \mathrm{bp}$ long fragment was fused through PCR to the uspA ORF, which was amplified with the primer pair CM161/CM2. For fusion-PCR primers CM2 and AMK168b were used and the resulting fragment encompasses 4,600 bp. The N-terminal half of yfp (nyfp) and $\operatorname{csnB}$ connected through a linker fused with PCR resulted in a 2,200 bp fragment, which was ligated into the Pmel site of the pME4313 plasmid containing cyfp::uspA fusion in its Swal site. Therefore, nyfp together with a linker was amplified 
from pCM31 using AMK163/CM94 and fused through PCR to csnB, which was amplified with the primers CM163/CM164. The resulting plasmid was named pME4708 and transformed into AGB1014 resulting in strain AGB1170.

The empty C-terminal half of the YFP (cyfp) amplified from pME4662 was ligated into the Swal cutting site of pME4313 and the nyfp::csnB fusion was ligated into the Pmel site for the control strains. Therefore, cyfp was amplified with AMK168b and CM165 from pME4662. The resulting plasmid is pME4709 and was transformed into AGB1014 resulting in AGB1171. Furthermore, for plasmid pME4710, cyfp::uspA was ligated into the Swal site and the empty nyfp was ligated into Pmel site. This plasmid was transformed into AGB1014 and resulted in the $A$. nidulans strain AGB1172.

\subsubsection{BiFC plasmid and strain construction of UspA and CsnF in A. nidulans}

cyfp::uspA gene fusion used for plasmid pME4708 was ligated into Swal site of pME4313. A fusion of nyfp and $c s n F$ was obtained through amplification of nyfp with AMK163/CM94 and amplification of $c s n F$ with CM166/CM167. These PCRs resulted in fragments of 500 bp and 1,300 bp, respectively. Both fragments were fused through another PCR reaction and ligated into the Pmel cutting site of the plasmid containing already cyfp::uspA. The plasmid was named pME4711 and ectopically integrated into AGB1014 resulting in strain AGB1173.

The control plasmid contained the nyfp::csnF fusion in the Pmel site of pME4313 and the empty cyfp in the Swal restriction site. This plasmid was named pME4712. Transformation of the circular plasmid into AGB1014 resulted in strain AGB1174.

\subsubsection{Construction of the RFP-CsnB plasmid and $A$. nidulans strain}

A plasmid containing a mcherry::csnB fusion was constructed in this study. Therefore, the 1,100 bp long 5' flanking region was amplified with the primer pair CM171/CM172. The 700 bp long mcherry gene was amplified from pAMK129 with primer CM173/CM174. Furthermore, primer CM175 and CM176 were used for the amplification of the csnB ORF. All three parts were combined through fusion-PCR reactions. The resulting product encompassed 3,500 bp and was ligated into the Pmll cutting site of pME4696. The corresponding 3' flanking region of $c s n B$ was amplified with primer pair CM177/CM178 and ligated into the Swal cutting site. The resulting plasmid was named pME4713. For transformation the whole construct was amplified with CM171/CM178 and as linear fragment transformed into AGB1159 resulting in strain AGB1175 and transformed into AGB1162 resulting in AGB1263. 


\subsubsection{Construction of the yeast-two-hybrid bait plasmid pME4715}

For the construction of pME4715 uspA was amplified from cDNA with primers CM128/CM129, which was cloned into the Notl restriction site of pME3229. The circular plasmid was transformed into the yeast strain EGY48.

Table 7: Aspergillus nidulans strains used in this study.

$\mathrm{P}=$ promotor, ${ }^{\mathrm{T}}=$ terminator, ${ }^{\text {comp }}=$ complementation, af $=$ Aspergillus fumigatus, bleo $=$ phleomycin

\begin{tabular}{|c|c|c|}
\hline Strain Name & Genotype & Reference \\
\hline AGB551 & $\Delta n k u A:: a r g B$, pyrG89, pyroA4, veA+ & (Bayram et al., 2012) \\
\hline AGB552 & $\Delta n k u A:: a r g B, p a b a A 1, y A 2, v e A+$ & (Bayram et al., 2012) \\
\hline AGB596 & ${ }^{P}$ gpdA::sgfp-phleo ${ }^{R} ;$ pabaA1, yA2, veA+ & (Bayram et al., 2012) \\
\hline AGB1092 & $\Delta n k u A:: a r g B$, pabaA1, yA2, veA+, lysA::six & this study \\
\hline AGB1093 & $\Delta n k u A:: a r g B, p a b a A 1, y A 2, v e A+$, lys $A^{\text {comp }}$ & this study \\
\hline AGB1151 & $\begin{array}{l}\Delta n k u A:: a r g B, \text { pabaA1, yA2, veA+, } \triangle \text { lys } A:: \text { six, } \\
\text { sgfp:csnD::six }\end{array}$ & this study \\
\hline AGB1152 & $\begin{array}{l}\Delta n k u A:: a r g B, \text { pabaA1, yA2, veA+, } \triangle l y s A:: \text { six, } \\
\text { sgfp::csnD::six, } \triangle \text { csnE::six }\end{array}$ & this study \\
\hline AGB1153 & $\begin{array}{l}\Delta n k u A:: a r g B, \text { pabaA1, yA2, veA+, } \triangle \text { lysA::six, } \\
\text { sgfp::csnD::six, csnE::six }\end{array}$ & this study \\
\hline AGB1154 & $\triangle n k u A:: a r g B, p a b a A 1, y A 2$, veA+, $\triangle \operatorname{argC}::$ six & this study \\
\hline AGB1155 & $\Delta n k u A:: a r g B, p a b a A 1, y A 2, v e A+, \arg C^{c o m p}$ & this study \\
\hline AGB1156 & $\Delta n k u A:: a r g B$, pyrG89, pyroA4, veA+, $\Delta u s p A:: p y r o A_{a f}$ & this study \\
\hline AGB1157 & 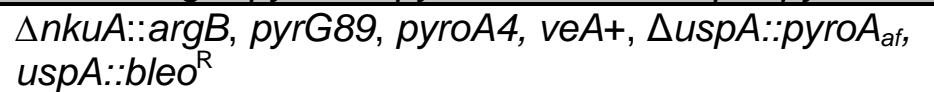 & this study \\
\hline AGB1158 & $\Delta n k u A:: a r g B$, pyrG89, pyroA4, veA+, $\Delta$ uspA::six & this study \\
\hline AGB1159 & $\begin{array}{l}\Delta n k u A:: a r g B, \text { pyrG89, pyroA4, veA+, } \\
\text { uspA::sgfp::: gpdA::nat }\end{array}$ & this study \\
\hline AGB1160 & $\begin{array}{l}\Delta n k u A:: a r g B, \text { pyrG89, pyroA4, veA+, } \\
\text { uspA::sgfp::PgpdA::nat }{ }^{\mathrm{R}} \text {, comp of } \Delta \text { uspA::six }\end{array}$ & this study \\
\hline AGB1161 & $\begin{array}{l}\text { unkuA::argB, pyrG89, pyroA4, veA+, } \\
\text { uspA::sgfp::: gpdA::nat, } \\
\text { gpdA:mrfp:h2A:his }{ }^{T}, \text { phleo }^{R}\end{array}$ & this study \\
\hline AGB1162 & $\Delta n k u A:: a r g B$, pyrG89, pyroA4, veA+, usp $A^{A A}:$ sgfp::six & this study \\
\hline AGB1163 & $\begin{array}{l}\text { DnkuA::argB, pyrG89, pyroA4, veA+, } \\
\text { uspA }{ }^{A A}:: \text { sgfp::six, }{ }^{P} \text { gpdA::mrfp::h2A::hisB }{ }^{T}: \text { nat }^{R} \\
\text { (pME3173) }\end{array}$ & this study \\
\hline AGB1164 & $\begin{array}{l}\triangle n k u A:: a r g B, \text { pyrG89, pyroA4, veA+, } \triangle \text { uspA::pyroA } A_{\text {af }}, \\
\text { veA::sgfp::six }\end{array}$ & this study \\
\hline AGB1165 & $\Delta n k u A:: a r g B$, pyrG89, pyroA4, veA+, veA::sgfp::six & (Thieme, 2018) \\
\hline AGB1166 & 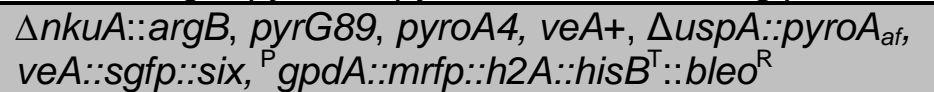 & this study \\
\hline AGB1167 & $\begin{array}{l}\Delta n k u A:: a r g B, \text { pyrG89, pyroA4, veA+, veA::sgfp:::six, } \\
{ }^{\mathrm{P}} \text { gpdA::mrfp::h2A::hisB }{ }^{\top}: \text { bleo }^{\mathrm{R}}\end{array}$ & this study \\
\hline
\end{tabular}


Table 7: continued.

\begin{tabular}{|c|c|c|}
\hline Strain Name & Genotype & Reference \\
\hline AGB1168 & $\begin{array}{l}\Delta n k u A:: a r g B, \text { pyrG89, pyroA4, veA+, } \triangle \text { uspA::pyro } A_{a f,} \\
\text { velB::gfp::six }\end{array}$ & this study \\
\hline AGB1132 & $\Delta n k u A:: a r g B$, pyroA4, pyrG89, ve $A_{+}$, velB::sgfp::six & (Thieme, 2018) \\
\hline AGB1169 & $\Delta n k u A:: a r g B$, pyrG89, pyroA4, veA+, $\triangle$ csnE::six & this study \\
\hline AGB1014 & $\begin{array}{l}\Delta n k u A:: a r g B, \text { pyrG89, pyroA4, } \\
\text { veA+, }{ }^{P} \text { gpdA::mrfp::h2A::hisB }{ }^{\top}:: \text { nat }^{\mathrm{R}}\end{array}$ & (Thieme, 2017) \\
\hline AGB1170 & 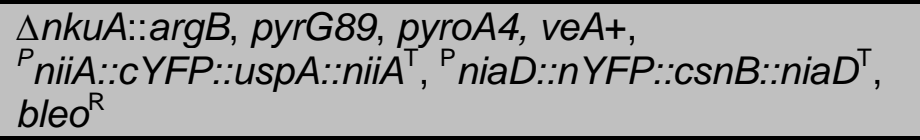 & this study \\
\hline AGB1171 & $\begin{array}{l}\Delta_{n k u A:: a r g B,} \text { pyrG89, pyroA4, veA+, } \\
P_{\text {niiA::cYFP::niiA }}{ }^{\top},{ }^{\mathrm{P}} \text { niaD::nYFP::csnB::niaD }{ }^{\top}, \text { bleo }^{\mathrm{R}}\end{array}$ & this study \\
\hline AGB1172 & $\begin{array}{l}\Delta_{\text {nkuA::argB, pyrG89, pyroA4, veA+, }} \\
{ }^{P} \text { niiA::cYFP::uspA::niiA }{ }^{\top},{ }^{P} \text { niaD::nYFP::niaD }{ }^{\top}, \text { bleo }^{\mathrm{R}}\end{array}$ & this study \\
\hline AGB1173 & $\begin{array}{l}\text { AnkuA::argB, pyrG89, pyroA4, veA+, } \\
P_{\text {niiA::cYFP::UspA::niiA }}{ }^{\top}{ }^{\mathrm{P}} \text { niaD::nYFP::csnF::niaD } \\
\text { bleo }^{\mathrm{R}}\end{array}$ & this study \\
\hline AGB1174 & $\begin{array}{l}\text { AnkuA::argB, pyrG89, pyroA4, veA+, }{ }^{P} \text { niiA:::cYFP:: } \\
\text { niiA }{ }^{\top},{ }^{\mathrm{P}} \text { niaD::nYFP::csnF::niaD }{ }^{\top} \text {, bleo }{ }^{\mathrm{R}}\end{array}$ & this study \\
\hline AGB1175 & $\begin{array}{l}\text { LnkuA::argB, pyrG89, pyroA4, veA+, } \\
\text { uspA::sgfp:: }{ }^{P} \text { gpdA::nat }{ }^{R}, \text { mcherry::csnB }\end{array}$ & this study \\
\hline AGB1263 & $\begin{array}{l}\text { InkuA::argB, pyrG89, pyroA4, veA+, } \\
\text { uspA } A^{A A}: \text { sgfp::six, mcherry::csnB }\end{array}$ & this study \\
\hline
\end{tabular}

\subsection{Genetic manipulations of microorganisms}

\subsubsection{Escherichia coli transformation}

The E. coli strain $\mathrm{DH} 5 \mathrm{a}$ was used for cloning reactions or propagation of plasmids. Transformations were performed as described elsewhere (Inoue et al., 1990). $200 \mu$ of the chemi-competent bacterial cells were thawed on ice. The DNA was mixed with the cells and incubated for 20 min on ice, followed by a heat shock at $42^{\circ} \mathrm{C}$ for $45 \mathrm{sec}$. After cooling down the cells for another $2 \mathrm{~min}$ on ice, $800 \mu \mathrm{LB}$ medium were added. Cells were incubated at $37^{\circ} \mathrm{C}$ under agitation for one hour to initiate growth of the cells before they were spread on an agar plate containing LB media with respective antibiotics. Agar plates were incubated overnight upside down at $37^{\circ} \mathrm{C}$ and clones were verified with colony PCRs.

\subsubsection{Aspergillus nidulans transformation}

Transformation of $A$. nidulans was performed as described previously (Punt et al., 1987). All strains used for transformations originated from wild types AGB551 or AGB552, which both carry 
the $\triangle n k u A$ mutation that allows integration of DNA fragments through homologous recombination more easily (Bayram et al., 2012; Nayak et al., 2006).

All buffers, solutions and materials were autoclaved or filtered and kept sterile and cold during the whole procedure. The $A$. nidulans host strain was grown vegetatively for $20 \mathrm{~h}$ at $37^{\circ} \mathrm{C}$ under agitation. Mycelium was harvested using sterile Miracloths (MERCK, Darmstadt, Germany) and washed with approximately $200 \mathrm{ml}$ of citrate buffer $(150 \mathrm{mM} \mathrm{KCl}, 580 \mathrm{mM} \mathrm{NaCl}, 50 \mathrm{mM} \mathrm{Na}$ citrate, $\mathrm{pH} 5.5$ was adjusted with citric acid). Mycelium was transferred with a sterile spatula into a sterile $300 \mathrm{ml}$ flask without indentations. $15 \mathrm{ml}$ of sterile protoplastation solution $(30 \mathrm{mg} / \mathrm{ml}$ Vinoflow $^{\circledR}$ Max or Vinotaste ${ }^{\circledR}$ Pro from NovozYMES (Bagsvaerd, Denmark) and $15 \mathrm{mg} / \mathrm{ml}$ lysozyme from Serva (Heidelberg, Germany) dissolved in citrate buffer) was added and carefully mixed. The solution was incubated for $105 \mathrm{~min}$ at $30^{\circ} \mathrm{C}$ under soft agitation at $75 \mathrm{rpm}$ to allow the digestion of the cell wall. Obtained protoplasts were filtered through Miracloth filters, washed with STC1700 buffer (1.2 M sorbitol, $10 \mathrm{mM}$ Tris pH5.5, $50 \mathrm{mM} \mathrm{CaCl}_{2}, 35 \mathrm{mM} \mathrm{NaCl}$ ) into precooled $50 \mathrm{ml}$ reaction tubes and incubated for $10 \mathrm{~min}$ on ice. Subsequently, protoplasts were pelleted during 12 min centrifugation at 2,500 rpm. The washing and centrifugation steps were repeated once. The resulting supernatant was discarded and the protoplasts resuspended in the last drops of STC1700 buffer before they were distributed equally into $15 \mathrm{ml}$ Falcons. Approximately $10 \mu \mathrm{g}$ of linearized DNA fragments or $5 \mu \mathrm{g}$ of circular plasmid DNA was added to the protoplasts, mixed cautiously and incubated for $25 \mathrm{~min}$ in ice. PEG4000 (10 mM Tris pH 7.5, $50 \mathrm{mM} \mathrm{CaCl}_{2}, 60 \%$ (w/v) polyethylene glycol (PEG) 4000) solution was added in three steps $(250 \mu \mathrm{l}, 250 \mu \mathrm{l}$ and $850 \mu \mathrm{l})$ with careful mixing in between to increase the chances of DNA uptake by the protoplasts during the following 20 min incubation step above ice. Finally, the solution was washed with ice-cold STC1700 and centrifuged at 2,500 rpm for $15 \mathrm{~min}$. The supernatant was discarded and the resulting protoplasts were resuspended in approximately $400 \mu \mathrm{l}$ remaining STC1700. $50 \mu \mathrm{l}, 200 \mu \mathrm{l}$ and the rest of the protoplast solution was mixed with preheated TOP agar $(0.7 \%(w / v)$ agar, 1.2 M sorbitol, $1 \%(w / v)$ glucose, 1x AspA, 2mM MgSO 4 , 1x trace elements) and distributed on agar plates containing MM with selection markers and $1.2 \mathrm{M}$ sorbitol. Plates were incubated for three to five days at $37^{\circ} \mathrm{C}$.

Resulting clones were picked and streaked out twice on selective medium before they were inoculated for genomic DNA preparation. Integration of DNA at the desired locus was verified by PCR and Southern hybridization. 


\subsubsection{Saccharomyces cerevisiae transformation}

Yeasts were transformed with plasmid DNA using the LiAc/SS Carrier DNA/PEG method (Ito et al., 1983). The yeast strain EGY48 was cultivated o/n at $30^{\circ} \mathrm{C}$ on a rotary shaker (FRÖBEL LABORTECHNIK GMBH, Lindau, Germany) in $10 \mathrm{ml}$ YPED medium. $400 \mu \mathrm{l}$ of this culture were used for inoculation of a fresh $10 \mathrm{ml}$ culture in YEPD medium. This culture was grown for five hours and all yeast cells were harvested by centrifugation. The cell pellet was washed twice with $10 \mathrm{~mL}$ of LiOAc/TE buffer (100 mM LiOAc, $1 \mathrm{mM}$ Tris-HCl pH 8.0, $0.1 \mathrm{mM}$ EDTA pH 8.0) to make cells competent. The pellet consisting of yeast cells was resuspended carefully in $400 \mu \mathrm{l}$ of LiOAc/TE buffer and distributed into two reaction tubes. SS carrier DNA (single stranded salmon sperm DNA) was incubated at $65^{\circ} \mathrm{C}$ for 5 min prior to the addition of $20 \mu \mathrm{l}$ of this solution to the yeast cells. Subsequently, 5-10 $\mu$ g plasmid DNA and $800 \mu \mathrm{l} 50 \%$ (w/v) PEG4000 were added. This mixture was incubated for $30 \mathrm{~min}$ at $30^{\circ} \mathrm{C}$, which was followed by a heat shock at $42^{\circ} \mathrm{C}$ for $25 \mathrm{~min}$. Yeast cells were pelleted through centrifugation for $1 \mathrm{~min}$ at 13,000 rpm and dissolved in $1 \mathrm{ml}$ fresh YPED medium. An incubation step for $1 \mathrm{~h}$ at $30^{\circ} \mathrm{C}$ followed prior to a final centrifugation step. The supernatant was discarded and yeast cells resuspended in the remaining medium inside the reaction tube. Cells were plated on selective SC medium, lacking a certain amino acid used for selection of yeast cells that took the plasmid DNA up. Incubation at $30^{\circ} \mathrm{C}$ for two to three days allowed the yeasts to grow. Colonies were streaked out once again on fresh SC media lacking the respective amino acid that serves as marker to select for positive clones.

\subsection{Southern hybridization}

Southern hybridization was performed to verify the integration of mutant constructs in the fungal genome (Southern, 1975). Fungal genomic DNA of the respective strains was digested with restriction enzymes. An enzyme with one recognition site in the linear DNA sequence, which was used for transformation, and another one in the neighboring DNA sequence to verify the integration of the DNA at the desired genomic locus, was used. The resulting DNA fragments should be significantly different between the parental and the newly generated strain.

The fungal genomic DNA was digested o/n with respective restriction enzymes and loaded on an agarose gel. Subsequently, agarose gels were washed under constant agitation for $10 \mathrm{~min}$ in buffer $1(0.25 \mathrm{M} \mathrm{HCl})$, for $20 \mathrm{~min}$ with buffer $2(0.5 \mathrm{M} \mathrm{NaOH}, 1.5 \mathrm{M} \mathrm{NaCl})$ and for 30 min with buffer $3(0.5 \mathrm{M}$ Tris, $1.5 \mathrm{M} \mathrm{NaCl}, \mathrm{pH}$ 7.4). The DNA in the agarose gel was transferred to Amersham ${ }^{\mathrm{TM}}$ Hybond $^{\mathrm{TM}}-\mathrm{N}$ membranes (GE HEALTHCARE) during dry-blotting at $r$ for at least 90 
$\min$. Afterwards, the membrane was dried for $8 \mathrm{~min}$ at $75^{\circ} \mathrm{C}$ and the DNA was crosslinked to the membrane through UV light exposure $(\lambda=254 \mathrm{~nm})$ for 2.5 min per side. Membranes were prehybridized in pre-hybridization buffer (purchased from GE HEALTHCARE and supplemented with a $0.5 \mathrm{M} \mathrm{NaCl}$ and $0.4 \%(\mathrm{w} / \mathrm{v})$ blocking reagent) for $30 \mathrm{~min}$ at $60^{\circ} \mathrm{C}$ in HERA hybrid $\mathrm{R}$ hybridization oven (HERAEUS INSTRUMENTS). After pre-hybridization, the DNA probe was added to the prehybridization buffer on the membrane and incubated $\mathrm{o} / \mathrm{n}$ at $60^{\circ} \mathrm{C}$ while rotating. The probe binds to a DNA sequence inside the fragment cut by the specific restriction enzyme. The minimum length of the probe was 700 bp to ensure specific DNA binding. Usually, the 5' or 3' flanking regions served as probe as they represent the flanking regions of the transformed DNA fragment and are therewith close to neighbouring gene sequences. The labelling of the probe was done according to manufacturer's instructions (GE HEALTHCARE). On the next day membranes were washed twice in post-hybridization buffer I $\left(1 \mathrm{mM} \mathrm{MgCl}_{2}, 3.5 \mathrm{mM}\right.$ SDS, $50 \mathrm{mM}$ sodium phosphate buffer, $150 \mathrm{mM} \mathrm{NaCl}, 2 \mathrm{M}$ Urea, $0.2 \%$ (w/v) blocking reagent) for $10 \mathrm{~min}$ at $60^{\circ} \mathrm{C}$ and twice in post-hybridization buffer II $(50 \mathrm{mM}$ Tris, $100 \mathrm{mM} \mathrm{NaCl}, \mathrm{pH} 10$ and freshly added $2 \mathrm{mM}$ $\mathrm{MgCl}_{2}$ ) for $5 \mathrm{~min}$ at rt under constant agitation. For detection of DNA bands $500 \mu$ of CDP-Star (GE HEALTHCARE) were applied and membranes were exposed to Amersham ${ }^{\mathrm{TM}}$ Hyperfilm $^{\mathrm{TM}} \mathrm{ECL}$ (GE HEALTHCARE).

\subsection{Morphological methods}

\subsubsection{Conidiospore quantification}

30,000 conidiospores were equally distributed on an agar plate containing $30 \mathrm{ml} \mathrm{MM}$. The plates were incubated at $37^{\circ} \mathrm{C}$ for four days. Subsequently, spores were harvested with a cotton stick and dissolved in $\mathrm{NaCl}$-Tween solution. The total number of conidiospores was determined using the Coulter Z2 particle counter (BECKMAN COULTER GMBH, Krefeld, Germany) or the Thoma cell counting chamber (hemocytometer) (PAUL MARIENFELD GMBH AND CO. KG, Lauda-Königshofen, Germany).

\subsubsection{Phenotypical characterization}

Phenotypical analyses of fungal strains were performed by point-inoculation of 5,000 spores on $30 \mathrm{ml}$ MM containing agar plates. Agar plates were incubated for four days at $37^{\circ} \mathrm{C}$ for inducing asexual development and for seven or 14 days for inducing sexual development. For photomicrographic pictures, 30,000 spores were distributed evenly on an agar plate containing 
$30 \mathrm{ml} \mathrm{MM}$ and incubated for the above mentioned time period. Photomicrographic pictures were obtained using the binocular microscope SZX12-ILLB2-200 (OLYMPUS DEUTSCHLAND GMBH, Hamburg, Germany). Pictures of squeezed cleistothecia were taken with Axiolab microscope (CARL ZeISS Microscopy GmBH, Jena, Germany). The OLYMPUS SC30 digital camera was used together with both microscopes. Pictures were edited with the cellSens software (OLYMPUS DEUTSCHLAND GMBH, Hamburg, Germany).

\subsection{Fluorescence microscopy}

Fluorescence microscopy was performed to investigate subcellular localization of proteins and to study protein interaction using bimolecular fluorescence complementation experiments (BiFC). Therefore, 2,000 spores were inoculated in $400 \mu \mathrm{l}$ minimal media on cover slides $(18 \times 18 \mathrm{~mm}$, TH. GEYER, Höxter, Germany) and incubated for $20 \mathrm{~h}$ at $37^{\circ} \mathrm{C}$. Afterwards, the medium was removed with a tissue and the cover slide was placed upside down on an object slide with $20 \mu \mathrm{l}$ fresh minimal medium. The cover slide was fixed using nail polish. The $A$. nidulans strains AGB551 or AGB1014 were included into the study to normalize the observed fluorescent signal against the background fluorescence. Nuclei were visualized through $r f p$ tagged $h 2 A$. In strains that expressed already an RFP tagged protein, nuclei were stained with Hoechst 33258 pentahydrate (Bis-benzimide) (INVITROGEN). Therefore, after $20 \mathrm{~h}$ of incubation at $37^{\circ} \mathrm{C}$, the minimal medium was exchanged by minimal medium supplemented with Hoechst (stock solution: $10 \mathrm{mg} / \mathrm{ml}$ in $\mathrm{dH}_{2} \mathrm{O}$ ) in a $1: 1,000$ dilution. Prior to microscopy, samples were incubated for another $15 \mathrm{~min}$ at $37^{\circ} \mathrm{C}$.

The Zeiss AxioObserver Z.1 inverted confocal microscope, equipped with Plan-Neofluar $63 x / 0.75$ (air), Plan-Apochromat $63 x / 1.4$ oil and a Plan-Apochromat 100x/1.4 oil objectives (ZEISS) and a QuantEM:512SC camera (PHOTOMETRICS, Tucson, AZ, USA) was used for fluorescence microscopical analyses. Pictures were taken and adjusted with the SlideBook 6.0 software package (INTELLIGENT IMAGING INNOVATIONS GMBH, Göttingen, Germany).

\subsection{Yeast-two-hybrid assay}

Protein interaction studies were performed with a yeast-two hybrid assay like described previously (Golemis et al., 1999). As background strain EGY48 was utilized, which harbors a LEU2 reporter system (Golemis et al., 2001). After successful transformation of bait (pJG202) and prey (pJG4-5) plasmids into the yeast strain, positive clones were inoculated in $2.5 \mathrm{ml} \mathrm{SC}$ medium $(0,15 \%(w / v)$ YNB-aa-as (yeast nitrogen base without amino acids and ammonium 
sulfate), $0.5 \%$ (w/v) ammonium sulfate, $0.2 \mathrm{mM}$ inositol, $0.2 \%(\mathrm{w} / \mathrm{v})$ amino acid powder mix and $2 \%(\mathrm{w} / \mathrm{v})$ raffinose) and grown $\mathrm{o} / \mathrm{n}$ at $30^{\circ} \mathrm{C}$ on a rotary shaker (Golemis et al., 2001). $\mathrm{OD}_{600}$ of yeast cells was measured with T80 UV/VIS Spectrometer (PG INSTRUMENTS, Lutterworth, UK) and adjusted to an $\mathrm{OD}_{600}$ of 0.1 with fresh SC medium. Agar plates containing SC medium with $2 \%(\mathrm{w} / \mathrm{v})$ glucose and $2 \%(\mathrm{w} / \mathrm{v})$ leucine served as positive control, whereas agar plates containing $2 \%(\mathrm{w} / \mathrm{v})$ glucose and no leucine served as negative control. Growth tests were performed on plates containing $2 \%(\mathrm{w} / \mathrm{v})$ galactose / $1 \%(\mathrm{w} / \mathrm{v})$ raffinose without leucine. In case of interacting proteins, the LEU2 reporter gene becomes activated in the presence of galactose. Five different dilutions of yeast cells were spotted on respective medium. Dilutions encompassed $\mathrm{OD}_{600}$ of 0.1 to 0.00001 , whereas cells were diluted with $\mathrm{dH}_{2} \mathrm{O}$. Different dilution of yeast strains were spotted in duplicates on the different media and incubated for three days at $30^{\circ} \mathrm{C}$.

\subsection{Protein methods}

\subsubsection{Isolation of protein crude extracts}

Liquid MM cultures were inoculated with $1 * 10^{6}$ spores $/ \mathrm{ml}$ and grown for $20 \mathrm{~h}$ under submerged conditions. For protein isolation of mycelia grown under asexual or sexual inducing conditions, mycelia from liquid cultures were shifted on agar plates containing $30 \mathrm{ml} \mathrm{MM}$ medium and incubated for up to $24 \mathrm{~h}$. Mycelia were harvested from liquid cultures through Miracloth filters or from solid media by scratching mycelia from the agar plates. Samples were frozen in liquid nitrogen immediately and grained manually. Approximately $300 \mu \mathrm{l}$ of grained mycelia was properly resuspended in Buffer $\mathrm{B}^{*}(300 \mathrm{mM} \mathrm{NaCl}, 100 \mathrm{mM}$ Tris $\mathrm{pH}$ 7.5, 10\% (v/v) glycerol, 0.5 mM EDTA, 0.05\% (v/v) NP-40, freshly supplemented with $1.5 \mathrm{mM}$ DTT, 1 tablet/50 ml complete EDTA-free protease inhibitor cocktail (ROCHE), $1 \mathrm{mM} \mathrm{PMSF}$ ) and centrifuged at 13,000 rpm for $30 \mathrm{~min}$ at $4^{\circ} \mathrm{C}$. The supernatant was transferred to a new reaction tube and centrifuged for another $10 \mathrm{~min}$ at $13,000 \mathrm{rpm}$ and $4^{\circ} \mathrm{C}$. After taking the supernatant into a clean tube again, the concentration was determined in 1:10 dilution using NanoDrop ND-1000 photospectrometer. If not indicated otherwise, $400 \mu \mathrm{g}$ of protein solution was prepared in a total volume of $40 \mu \mathrm{l}$ and mixed with $20 \mu \mathrm{l}$ of SDS sample buffer $(250 \mathrm{mM}$ Tris- $\mathrm{HCl} \mathrm{pH} 6.8,15 \%$ (v/v) $\beta$-mercaptoethanol, $30 \%(\mathrm{v} / \mathrm{v})$ glycerol, $7 \%(\mathrm{v} / \mathrm{v})$ SDS, $0.3 \%(\mathrm{w} / \mathrm{v})$ bromophenol blue). Protein samples were denatured while boiling at $95^{\circ} \mathrm{C}$ for $10 \mathrm{~min}$. 


\subsubsection{Sodium dodecyl sulfate polyacrylamide gel electrophoresis and western hybridization}

Proteins were separated by their molecular weight using sodium dodecyl sulfate polyacrylamide gel electrophoresis (SDS-PAGE) while applying an electrical field. Thereby, small molecular weight proteins migrate faster through the polyacrylamide gel than proteins with high molecular weight (Laemmli, 1970). SDS gels consist of a short stacking gel (3.67 $\mathrm{ml} \mathrm{H}_{2} \mathrm{O}, 625 \mu \mathrm{l} 1 \mathrm{M}$ Tris pH 6.8, $30 \mu \mathrm{l}$ 10\% (w/v) SDS, $650 \mu \mathrm{l} 30 \%$ (v/v) acrylamide, $5 \mu \mathrm{l}$ TEMED, $25 \mu \mathrm{l}$ 10\% (w/v) APS) and a longer separation gel $\left(2.8 \mathrm{ml} \mathrm{H}_{2} \mathrm{O}, 3.75 \mathrm{ml} 1 \mathrm{M}\right.$ Tris $\mathrm{pH} 8.8,100 \mu \mathrm{l} 10 \%$ (w/v) SDS, $3.3 \mathrm{ml}$ $30 \%(\mathrm{v} / \mathrm{v}$ ) acrylamide, $10 \mu \mathrm{l}$ TEMED, $50 \mu \mathrm{l}$ 10\% (w/v) APS), which differ in their acrylamide concentration. The running buffer consisted of $25 \mathrm{mM}$ Tris, $0.25 \mathrm{M}$ glycine, $0.1 \%(\mathrm{w} / \mathrm{v}) \mathrm{SDS}$. The gels were afterwards used for western hybridization experiments.

During western hybridization experiments, the proteins on the gel were transferred on a nitrocellulose membrane (Amersham ${ }^{\mathrm{TM}}$ Protran $^{\mathrm{TM}} 0.45 \mu \mathrm{m}$ NC nitrocellulose membranes (GE Healthcare)) in SDS-PAGE chambers either overnight at $35 \mathrm{~V}$ at rt or ice-cooled for up to $2 \mathrm{~h}$ at $100 \mathrm{~V}$ in transfer buffer (25 mM Tris, $192 \mathrm{mM}$ glycine, 0.02\% (w/v) SDS) (Towbin et al., 1979). Subsequently, membranes were stained with Ponceau S (3\% (v/v) trichloroacetic acid, $0.2 \%(w / v)$ Ponceau S) to verify the transfer of the proteins onto the membrane (Romero-Calvo et al., 2010).

All following steps were performed under constant agitation. First, the membranes were blocked for one hour in $5 \%(\mathrm{w} / \mathrm{v})$ milk powder in TBS-T (10 mM Tris- $\mathrm{HCl} \mathrm{pH} 8.0,150 \mathrm{mM} \mathrm{NaCl}, 0.05 \%$ (v/v) Tween 20) and afterwards incubated with primary antibody for two hours at rt or at $4^{\circ} \mathrm{C} \mathrm{o} / \mathrm{n}$. As primary antibodies $\alpha-$-GFP antibody in 1:500 (sc-9996, SANTA CRUZ BIOTECHNOLOGY, Dallas, TX, USA), $\alpha$-CsnE in 1:2,000 (GENESCRIPT, New Jersey, USA) or $\alpha$-Ubiquitin in 1: 2,000 (clone P4D1-A11, MERCK MILLIPORE, Darmstadt, Germany) dilutions in $5 \%$ (w/v) milk powder dissolved in TBS-T were used. As secondary antibodies a-mouse (115-035-003, JACKSON IMMUNO RESEARCH, West Grove, CA, USA) or $\alpha$-rabbit (G21234, INVITROGEN) in 1:2,000 dilutions in $5 \%$ (w/v) milk powder in TBS-T solution were used. Secondary antibodies were applied for approximately two hours. After the incubation with primary and secondary antibody the membrane was washed three times for $10 \mathrm{~min}$ with TBS-T solution. For detection of chemiluminescent signals an 1:1 mixture of detection solution $\mathrm{A}(2.5 \mu \mathrm{M}$ luminol, $400 \mu \mathrm{M}$ paracoumarat, $100 \mathrm{mM}$ Tris- $\mathrm{HCl} \mathrm{pH}$ 8.5) and detection solution $\mathrm{B}\left(5.4 \mathrm{mM} \mathrm{H}_{2} \mathrm{O}_{2}, 100 \mathrm{mM}\right.$ Tris$\mathrm{HCl} \mathrm{pH}$ 8.5) was incubated for $90 \mathrm{sec}$ in the dark. Signals were detected with a Fusion-SL7 chemiluminescence detection system (PEQLAB, Erlangen, Germany) and pictures were recorded with the Fusion 15.15 software from VILBER LOURMAT (Marne-la-Vallée cedex 3, France). 


\subsubsection{GFP pull down}

For GFP pull downs fungal strains were inoculated with $1 * 10^{6}$ spores $/ \mathrm{ml}$ in $500 \mathrm{ml}$ liquid MM and grown for $20 \mathrm{~h}$ at $37^{\circ} \mathrm{C}$ under agitation. Strains were harvested through Miracloth filters and washed with approximately $200 \mathrm{ml} 0.96 \%$ (w/v) NaCl solution, which was freshly supplemented with $1 \mathrm{mM}$ PMSF and $1 \%(\mathrm{v} / \mathrm{v})$ DMSO. Equal amounts of mycelial samples were manually grained and intensively dissolved in Buffer $\mathrm{B}^{*}$ (freshly supplemented with $1.5 \mathrm{mM}$ DTT, 1 tablet/50 ml complete EDTA-free protease inhibitor cocktail (ROCHE), $1 \mathrm{mM}$ PMSF and a 1:1,000 dilution of a phosphatase inhibitor mix $(1 \mathrm{mM} \mathrm{NaF}, 0.5 \mathrm{mM}$ sodium-orthovanadate, $8 \mathrm{mM}$ B-glycerolphosphate disodium pentahydrate)). Samples were centrifuged for $1 \mathrm{~h}$ at $15,000 \mathrm{rpm}$ in SS34 centrifuge tubes (THERMO FISHER SCIENTIFIC) at $4^{\circ} \mathrm{C}$ in the Sorvall RC-5B Plus Refrigerated Centrifuge from THERMO FISHER SCIENTIFIC. The supernatant containing the proteins was filtered through $0.2 \mu \mathrm{m}$ sterile filters (SARSTEDT). Approximately $5 \mathrm{ml}$ supernatant were incubated with $60 \mu$ pre-equilibrated GFP-Trap ${ }^{\circledR}$ A beads from CHROMOTEK (PlaneggMartinsried, Germany) with freshly supplemented Buffer $B^{*}$ in Poly-Prep $\AA$ Chromatography Columns (BIO-RAD, Hercules, CA, USA) for two hours at $4^{\circ} \mathrm{C}$ on a rotator. Subsequently, the crude extracts were passed through the polypropylene column and the GFP beads with the bound target proteins stayed inside the column. Two washing steps with twice $1 \mathrm{ml}$ washing buffer I (300 mM NaCl, $10 \mathrm{mM}$ Tris $\mathrm{pH}$ 7.5, $0.5 \mathrm{mM}$ EDTA, freshly supplemented with 1mM PMSF, $1.5 \mathrm{mM}$ DTT, 1 tablet/50 ml complete EDTA-free protease inhibitor cocktail) and washing buffer II (500 mM NaCl, $10 \mathrm{mM}$ Tris $\mathrm{pH}$ 7.5, $0.5 \mathrm{mM}$ EDTA, freshly supplemented with 1mM PMSF, $1.5 \mathrm{mM}$ DTT, 1 tablet/50 ml complete EDTA-free protease inhibitor cocktail) was performed, respectively. Elution of the proteins was achieved by mixing $150 \mu 0.2 \mathrm{M}$ glycine $\mathrm{pH} 2.5$ for $30 \mathrm{sec}$ with the proteins bound by the GFP beads. The elution fraction was collected in a LoBind $1.5 \mathrm{ml}$ reaction tube, which contained $15 \mu \mathrm{l} 1 \mathrm{M}$ Tris $\mathrm{pH} 10.4$ for neutralization. Three elution steps were performed before the GFP beads were washed with $\mathrm{dH}_{2} \mathrm{O}$ and 20 $\%(v / v)$ ethanol for re-using. The concentration of the elution fractions was determined with a NanoDrop ND-1000 photospectrometer (PEQLAB). Samples were either boiled for applying on denaturing SDS-PAGE or were submitted to chloroform/methanol precipitation for subsequent in-solution tryptic digestion. 


\subsection{Sample preparation for mass spectrometric analysis}

\subsubsection{Trypsin in-gel digestion}

Trypsin in-gel digestion was performed to digest proteins into small peptides and make them accessible for LC/MS-MS analyses. Pipette tips prepared with vinyl gloves, vinyl gloves and LoBind reaction tubes have been used for the whole procedure. All incubation steps during this procedure were done during constant agitation at rt if not indicated otherwise. The volume of the solution was dependent on the amount of gel pieces in the reaction tube. The gel pieces had to be completely covered with the respective solvent. In-gel digestion was applied for all SILAC samples analyzed in this study.

Trypsin in-gel digestion was performed as previously described (Shevchenko et al., 2007). Protein samples were applied to a denaturing SDS-PAGE, stained with Coomassie Brilliant Blue (40\% (v/v) ethanol, $10 \%(\mathrm{v} / \mathrm{v})$ acetic acid, 0.05\% (w/v) Coomassie R-250 (SERVA)) and destained with destaining solution (12.5\% (v/v) isopropanol, 10\% (v/v) acetic acid). The SDS-gel was cut into approximately $2 \mathrm{~mm}$ big pieces and incubated with acetonitrile for $10 \mathrm{~min}$ followed by $10 \mathrm{~min}$ drying in the SpeedVac concentrator (THERMO FISHER SCIENTIFIC). Addition of $10 \mathrm{mM}$ DTT and incubation at $56^{\circ} \mathrm{C}$ on a heating block FOR $1 \mathrm{~h}$ without shaking was performed to denature and reduce disulfide bonds of the proteins. Subsequently, 45 min incubation with $150 \mathrm{mM}$ lodoacetamide was done to alkylate the cysteine residues. Three washing cycles with alternating $100 \mathrm{mM} \mathrm{NH}_{4} \mathrm{HCO}_{3}$ and acetonitrile for $10 \mathrm{~min}$ respectively followed. Prior to the addition of trypsin enzyme, the gel pieces were dried in the SpeedVac for $10 \mathrm{~min}$ at $50^{\circ} \mathrm{C}$. Samples were afterwards incubated on ice with trypsin digestion buffer (Trypsin NB Sequencing Grade (PROMEGA) prepared according to manufacturer's specifications) for $45 \mathrm{~min}$. In this time, trypsin digestion buffer should be absorbed by the gel pieces; remaining buffer was discarded. Addition of $25 \mathrm{mM} \mathrm{NH}_{4} \mathrm{HCO}_{3}$ and incubation o/n at $37^{\circ} \mathrm{C}$ without shaking allowed the digestion of the proteins into small peptides.

Extraction of peptides from the gel pieces was performed on the next day. Thereby, the supernatants were all collected in a fresh collection tube. Incubation of gel pieces for 10 min with $25 \mathrm{mM} \mathrm{NH}_{4} \mathrm{HCO}_{3}$ allowed the extraction of acidic peptides. Remaining peptides were isolated from the gel pieces by three times washing with a solution containing $50 \%(\mathrm{v} / \mathrm{v})$ acetonitrile and $5 \%$ (v/v) formic acid for $20 \mathrm{~min}$. Collected supernatants were completely dried in the SpeedVac and the protein pellet was stored at rt or directly used for further Stage Tip purifications. 


\subsubsection{Trypsin in-solution digestion}

For in-solution tryptic digestion the proteins were not separated on a denaturing SDS-PAGE, but precipitated using chloroform/methanol extraction to prepare them for further digestion. Insolution digestion was applied for GFP pull down samples.

\subsubsection{Chloroform/methanol extraction}

Chloroform/methanol extraction was performed to precipitate proteins in the elution fraction of the GFP pull downs to process them further with in-solution enzymatic digestion. Therefore, $400 \mu \mathrm{l}$ methanol were added to $100 \mu \mathrm{l}$ of the elution fraction. The solution was mixed by vortexing and centrifuged for $10 \mathrm{sec}$ at $10,000 \mathrm{rpm}$. Subsequently, $100 \mu \mathrm{l}$ chloroform were added and mixing and centrifugation steps were repeated. Afterwards, $300 \mu \mathrm{d} \mathrm{H}_{2} \mathrm{O}$ were added and the solution was thoroughly mixed by vortexing for $30 \mathrm{sec}$. Centrifugation for $3 \mathrm{~min}$ at $10,000 \mathrm{rpm}$ and $4^{\circ} \mathrm{C}$ should result in a phase separation. The upper phase was discarded without disturbing the interface where the proteins are located. Finally, $300 \mu$ methanol were added to the lower phase, the solution was vortexted and centrifuged for $10 \mathrm{~min}$ at 13,000 rpm at $4^{\circ} \mathrm{C}$. The supernatant was discarded as the proteins precipitate and form a pellet. This pellet was air-dried o/n prior to the RapiGest in-solution digestion.

\subsubsection{RapiGest ${ }^{\mathrm{TM}}$ in-solution digestion}

RapiGest $^{\text {TM }}$ SF was purchased from WATERS GMBH (Eschborn, Germany) and used according to manufacturer's instructions. Briefly, the air-dried protein pellet was dissolved in $40 \mu l$ $0.1 \%(\mathrm{w} / \mathrm{v})$ RapiGest ${ }^{\mathrm{TM}}$ surfactant (SF) by thoroughly pipetting up and down. A final concentration of $5 \mathrm{mM}$ DTT was added and the samples were incubated for $30 \mathrm{~min}$ at $60^{\circ} \mathrm{C}$. Afterwards, the samples were cooled down to rt prior to the addition of $15 \mathrm{mM}$ lodoacetamide. An incubation step for $30 \mathrm{~min}$ in darkness at rt followed. Trypsin digestion buffer, which is identical with the one used for in-gel digestion, was applied to the solution in a 1:20 dilution. Samples were incubated $\mathrm{o} / \mathrm{n}$ at $37^{\circ} \mathrm{C}$ to allow the processing of the proteins. On the following day, RapiGest surfactant was precipitated by the addition of $0.5 \%$ (v/v) trifluoracetic acid (TFA). This should reduce the $\mathrm{pH}$ to 2 , which was verified by applying a drop of the solution on $\mathrm{pH}$ paper. The solution was incubated for $45 \mathrm{~min}$ at $37^{\circ} \mathrm{C}$ and subsequently centrifuged at $13,000 \mathrm{rpm}$ for $10 \mathrm{~min}$. Supernatant was transferred into a clean reaction tube and dried out in the SpeedVac for further purification steps. 


\subsubsection{C18 Stage Tip Purification}

Stop and Go Extraction (Stage) Tip Purification was performed prior to MS analyses to remove salts and other contaminants from the peptides (Rappsilber et al., 2003). Therefore, peptide pellets resulting from tryptic digestions were resuspended in sample buffer $\left(98 \%(\mathrm{v} / \mathrm{v}) \mathrm{ddH}_{2} \mathrm{O}\right.$, $2 \%(\mathrm{v} / \mathrm{v})$ acetonitrile and $0.1 \%(\mathrm{v} / \mathrm{v})$ formic acid) by thoroughly pipetting and by incubation in an ultrasonic bath for $3 \mathrm{~min}$ at maximum power. C18 (reversed phase material) columns were prepared by putting two to three layers C18 material plugs into $200 \mu$ pipet tips. C18 material was pushed as much to the bottom to the tip as possible to make sure that there is no free space between the material and the pipet tip wall anymore. Resulting Stage Tips were equilibrated with $100 \mu \mathrm{l} 0.1 \%(\mathrm{v} / \mathrm{v})$ formic acid in HPLC grade methanol. After addition of the solution, the Stage Tips were centrifuged at 10,000 rpm for 2 min. Further, a $100 \mu \mathrm{l} 70 \%$ (v/v) acetonitrile containing $0.1 \%(\mathrm{v} / \mathrm{v})$ formic acid solution was applied to the $\mathrm{C} 18$ material. Final washing steps were performed with $100 \mu \mathrm{l} 0.1 \%(\mathrm{v} / \mathrm{v})$ formic acid in $\mathrm{dH}_{2} \mathrm{O}$, respectively. Centrifugation allowed the solutions to flow through the column material. After successful equilibration of C18 material, the peptide solution dissolved in sample buffer was applied to the columns. This should be directly in contact with the C18 material without any air bubbles in between. The peptide solution was incubated for 5 minutes on the column. Prior to 5 min centrifugation at 3,000 rpm the Stage Tip was placed into a new collection tube to allow reloading of the flow through once. Peptides bind to the $\mathrm{C} 18$ material and washing steps with $100 \mu \mathrm{l} 0.1 \%(\mathrm{v} / \mathrm{v})$ formic acid in $\mathrm{dH}_{2} \mathrm{O}$ should remove salts and contaminants. For the final elution step the Stage Tips were again transferred to a clean reaction tube. Elution was done by adding two times $30 \mu \mathrm{l} 70 \%(\mathrm{v} / \mathrm{v})$ acetonitrile containing $0.1 \%(\mathrm{v} / \mathrm{v})$ formic acid. Eluted peptides were again dried completely in the SpeedVac at $50^{\circ} \mathrm{C}$.

For final MS measurements, peptides were dissolved in $20 \mu \mathrm{l}$ sample buffer. The solution was transferred into glass mass spectrometry vials (AGILENT TECHNOLOGIES, California, USA).

\subsubsection{Liquid chromatography / mass spectrometry - mass spectrometry (LC/MS-MS)}

LC/MS-MS analyses were performed with the Orbitrap Velos Pro mass spectrometer (THERMO FISHER SCIENTIFIC) and the RSLCnano Ultimate 3000 chromatography system (THERMO FISHER SCIENTIFIC).

Atfer C18 Stage Tip purification, peptides were dissolved in $20 \mu \mathrm{l}$ sample buffer (98\% (v/v) $\mathrm{ddH}_{2} \mathrm{O}, 2 \%(\mathrm{v} / \mathrm{v})$ acetonitrile, $0.1 \%(\mathrm{v} / \mathrm{v})$ formic acid). Peptides were separated by reverse phase chromatography on an Acclaim PepMap RSLC column (THERMO FISHER SCIENTIFIC) with 
a water-acetonitrile gradient. Eluted peptides were online ionized by nano-electrospray at $2.4 \mathrm{kV}$ utilizing the Nanospray Flex Ion Source (THERMO FISHER SCIENTIFIC). Full scans with the mass range of $300-1,850 \mathrm{~m} / \mathrm{z}$ were acquired with the Orbitrap-FT analyzer (THERMO FISHER SCIENTIFIC) at a resolution of 30,000 for GFP pull down experiments and 60,000 for SILAC based experiments. In parallel, collision-induced dissociation (CID) fragmentation of data dependent top-ten peptides was performed with the LTQ Velos Pro ion trap (THERMO FISHER SCIENTIFIC). For acquisition of mass spectra and programming of the LC/MS-MS method the XCalibur 2.2 ${ }^{\mathrm{TM}}$ software (THERMO FISHER SCIENTIFIC) was used.

\subsubsection{Data analysis with MaxQuant and Perseus}

Data analysis for the SILAC amino acid incorporation was performed with Proteome Discoverer $^{\text {TM }} 1.4$ (THERMO FISHER SCIENTIFIC) using the SequestHT ${ }^{\mathrm{TM}}$ and Mascot ${ }^{\mathrm{TM}}$ search algorithms against an Aspergillus nidulans specific database, which encompasses common contaminants. For all other experiments data analyses was done with the MaxQuant version 1.5.1.0 in combination with Perseus 1.5.0.15 (Cox and Mann, 2008; Tyanova et al., 2016).

Preset MaxQuant default parameters have been used for the data analyses. For the SILAC experiment, a standard LC/MS run with multiplicity 3 was set to enable the choice of isotopicallylabeled amino acids. Lys4 and Lys8 were chosen as medium and heavy label, respectively. The label free quantification (LFQ) and the re-quantify option was enabled in the Global Parameters tab. The A. nidulans database was downloaded from Uniprot (ID: AUP000000560) in 2014. The workflow used for SILAC data evaluation is depicted in Table 8.

Normalized SILAC ratios were used for further analysis. The regulation threshold was set to $\log _{2}$ SILAC ratio of \pm 0.5 . Only proteins, which were regulated in $\triangle \operatorname{csn} E$ compared to the $\Delta / y s A$ background and $c s n E^{c o m p}$ strains were considered in the analyses. Furthermore, the threshold had to be reached in two out of three biological replicates.

Table 8: Workflow of SILAC data processing with Perseus.

\begin{tabular}{|l|l|l|}
\hline Step & Command & Description \\
\hline $\mathbf{1}$ & Generic matrix upload & $\begin{array}{l}\text { proteinGroups.txt } \\
\text { normalized SILAC ratios, Unique Peptides, Protein IDs, } \\
\text { Potential Contaminants, Reverse, Only identified by site }\end{array}$ \\
\hline $\mathbf{2}$ & $\begin{array}{l}\text { Filter rows based on } \\
\text { categorical column }\end{array}$ & $\begin{array}{l}\text { Filter for Potential Contaminants, Reverse, Only identified } \\
\text { by site and remove rows with "+" }\end{array}$ \\
\hline $\mathbf{3}$ & Remove empty columns & $\begin{array}{l}\text { Potential Contaminants, Reverse, Only identified by site } \\
\text { columns are removed }\end{array}$ \\
\hline
\end{tabular}


Table 8: continued.

\begin{tabular}{|c|c|c|}
\hline Step & Command & Description \\
\hline 4 & Transform & $\begin{array}{l}\text { Inverse SILAC ratios }(1 / x) \text { to place values for control } \\
\text { strains in the denominator }\end{array}$ \\
\hline 5 & Transform & $\log _{2}(X)$ of all expression columns \\
\hline 6 & Visualization $\rightarrow$ Histograms & $\begin{array}{l}\text { To confirm that values follow a normal distribution and } \\
\text { evaluate if further normalization steps are required }\end{array}$ \\
\hline 7 & $\begin{array}{l}\text { Filter rows based on valid } \\
\text { values }\end{array}$ & $\begin{array}{l}\text { Values should be valid for at least six out of nine } \\
\text { expression columns }\end{array}$ \\
\hline 8 & Imputation & Impute missing values from normal distribution \\
\hline 9 & Categorical annotation rows & Define Group 1 for control $c s n E^{c o m p} / \Delta / y s A$ of replicate 1 \\
\hline 10 & $\begin{array}{l}\text { Filter rows based on valid } \\
\text { values }\end{array}$ & $\begin{array}{l}\text { In Group } 1 \text { values should be between }-0.5 \text { and } 0.5 \text {, all } \\
\text { other rows are excluded from further analysis }\end{array}$ \\
\hline 11 & Categorical annotation rows & Define Group 2 for control $c s n E^{c o m p} / \Delta / y s A$ of replicate 2 \\
\hline 12 & $\begin{array}{l}\text { Filter rows based on valid } \\
\text { values }\end{array}$ & $\begin{array}{l}\text { In Group } 2 \text { values should be between }-0.5 \text { and } 0.5 \text {, all } \\
\text { other rows are excluded from further analysis }\end{array}$ \\
\hline 13 & Categorical annotation rows & Define Group 3 for control $c s n E^{c o m p} / \Delta / y s A$ of replicate 3 \\
\hline 14 & $\begin{array}{l}\text { Filter rows based on valid } \\
\text { values }\end{array}$ & $\begin{array}{l}\text { In Group } 3 \text { values should be between }-0.5 \text { and } 0.5 \text {, all } \\
\text { other rows are excluded from further analysis }\end{array}$ \\
\hline 15 & Add annotation & Aspergillus nidulans database derived from UniProt \\
\hline 16 & Statistics Rows & Mean of $\Delta c s n E / c s n E^{c o m p}$ replicate I, 2 and 3 \\
\hline 17 & Statistics Rows & Mean of $\Delta c s n E / \Delta / y s A$ replicate I, 2 and 3 \\
\hline 18 & Statistics Rows & Mean of $c s n E^{\text {comp }} / \Delta / y s A$ replicate I, 2 and 3 \\
\hline 19 & Change column type & $\begin{array}{l}\text { Mean } \Delta c s n E / c s n E^{c o m p}, \Delta c s n E / \Delta l y s A \text { and } c s n E^{c o m p} / \Delta l y s A \\
\text { from numerical to expression }\end{array}$ \\
\hline 20 & Visualization & Scatter Plot Mean $\Delta c s n E / c s n E^{c o m p}$ and $\Delta c s n E l \Delta l y s A$ \\
\hline
\end{tabular}

Data analyses for GFP pull down experiments were performed with the default MaxQuant settings, the label free quantification was additionally enabled. The $A$. nidulans protein database from UniProt was used, which is the same as the one used for the SILAC experiments. Downstream analyses were performed with the Perseus software; the workflow is given in Table 9. Thereby, logarithmized LFQ intensities and the number of unique peptides were applied as filter categories. All proteins that were identified in the GFP control strain were excluded from further analyses. For the UspA-GFP pull down experiments proteins had to have $\log _{2} L F Q$ intensity of 21 and at least two unique peptides in at least two out of three biological replicates to be considered for further analysis. Proteins that were identified at least twice in three biological replicates have been considered as putative interaction partners. Proteins only present in UspA ${ }^{\text {AA }}$-GFP, only in UspA-GFP or pulled with both versions were selected manually from the Numeric venn diagram. 
Table 9: Workflow for GFP pull down analyses with the Perseus Software.

\begin{tabular}{|c|c|c|}
\hline Step & Command & Description \\
\hline 1 & Generic matrix upload & $\begin{array}{l}\text { proteinGroups.txt } \\
\text { LFQ intensities, Unique Peptides, Protein IDs, Potential } \\
\text { Contaminants, Reverse, Only identified by site }\end{array}$ \\
\hline 2 & $\begin{array}{l}\text { Filter rows based on } \\
\text { categorical column }\end{array}$ & $\begin{array}{l}\text { Filter for Potential Contaminants, Reverse, Only } \\
\text { identified by site and remove rows with "+" }\end{array}$ \\
\hline 3 & Remove empty columns & $\begin{array}{l}\text { Potential Contaminants, Reverse, Only identified by site } \\
\text { columns are removed }\end{array}$ \\
\hline 4 & Transform & $\log _{2}(X)$ of all expression columns \\
\hline 5 & Imputation & Impute missing values from normal distribution \\
\hline 6 & Add annotation & Aspergillus nidulans database derived from UniProt \\
\hline 7 & Change column type & $\begin{array}{l}\text { Unique peptides GFP control 1-3 from Numerical } \rightarrow \\
\text { Expression }\end{array}$ \\
\hline 8 & Categorical annotation rows & $\begin{array}{l}\text { Define Group } 1 \text { for Unique Peptides GFP control } \\
\text { replicate } 1\end{array}$ \\
\hline 9 & $\begin{array}{l}\text { Filter rows based on valid } \\
\text { values }\end{array}$ & In Group 1 values should be 1 or less \\
\hline 10 & Categorical annotation rows & $\begin{array}{l}\text { Define Group } 2 \text { for Unique Peptides GFP control } \\
\text { replicate } 2\end{array}$ \\
\hline 11 & $\begin{array}{l}\text { Filter rows based on valid } \\
\text { values }\end{array}$ & In Group 2 values should be 1 or less \\
\hline 10 & Categorical annotation rows & $\begin{array}{l}\text { Define Group } 3 \text { for Unique Peptides GFP control } \\
\text { replicate } 3\end{array}$ \\
\hline 11 & $\begin{array}{l}\text { Filter rows based on valid } \\
\text { values }\end{array}$ & In Group 3 values should be 1 or less \\
\hline 12 & Imputation & Replace imputed values by $\mathrm{NaN}$ \\
\hline 13 & Misc. & Numeric Venn Diagram \\
\hline
\end{tabular}




\section{Results}

\subsection{The COP9 signalosome mediates proteome changes during vegetative, filamentous growth of $A$. nidulans}

The COP9 signalosome enables the exchange of the receptor complexes of E3 ubiquitin ligases by deneddylating the cullin scaffold protein (Choo et al., 2011; Mosadeghi et al., 2016). The intrinsic deneddylation activity is conferred through the catalytically active subunit CsnE (Lyapina et al., 2001). Removal of the Nedd8 from the cullin protein leads to structural rearrangements, which finally enable the disassociation of the bound receptor complex and the ability to bind another one. This ensures specific labeling of substrates with ubiquitin molecules. Previously performed transcriptome analysis in an A. nidulans strain defective in the COP9 signalosome function revealed that $2.5 \%$ of the annotated genes are differentially regulated in the absence of the catalytically active subunit CsnE during vegetative development (Nahlik et al., 2010). Throughout the whole fungal development $15.4 \%$ of the genes were differentially regulated (Nahlik et al., 2010). Through its deneddylation activity the COP9 signalosome is rather involved in controlling protein half-life than gene transcription.

Controlled protein degradation through the UPS is a highly dynamic process. If the impairment in deneddylation by a dysfunctional COP9 signalosome affects already fungal protein half-life during vegetative, hyphal growth in liquid media or if this is rather required for formation of the complex multicellular structures was analyzed in this study.

The changes in the fungal proteome after the achievement of developmental competence were examined in the presence or absence of a functional COP9 signalosome. Several MS-based methods have been established to quantify protein abundances in different cultures or cell lines. Originally, proteomes were analyzed with 2D gel electrophoresis, where proteins are separated according to molecular weight and their isoelectric point and subsequently identified through mass spectrometry. A disadvantage of this method is the limitation in the amount of proteins that can be detected as only high abundant proteins are suitable to be identified (Gygi et al., 2000; Rabilloud and Lelong, 2011). Relative protein quantification utilizing the isotope-coded affinity tag (ICAT) labeling method enables labeling of cysteine residues with tags harboring different number of deuterium isotopes, which makes them distinguishable by their mass in mass spectrometry analyses (Aebersold, 2003). A number of different isobaric mass tags such as tandem mass tag (TMT) or different issobaric tags for relative and absolute protein quantification (iTRAQ) were developed in recent years, which enable the study of up to four or even eight 
different conditions (Rauniyar and Yates, 2014). Another possibility to quantify changes in protein abundances is the metabolic labeling approach with different isotopically-labeled amino acids like stable isotope labeling with amino acids in cell culture (SILAC) (Lau et al., 2014; Ong et al., 2002). Thereby, samples deriving from different cultures can be combined in early steps of sample preparation. This leads to higher accuracy and reproducibility of the obtained results (Lau et al., 2014). Furthermore, the use of essential amino acids ensured that all peptides contain the differentially isotopically-labeled amino acid isotopes (Lau et al., 2014; Ong et al., 2002). SILAC is mainly based on isotopically-labeled L-lysine and L-arginine amino acids and is used in cell culture systems and in S. cerevisiae (de Godoy et al., 2006; Lau et al., 2014). SILAC was established for the use for filamentous fungi such as $A$. nidulans in this study.

\subsubsection{Establishing of a stable isotope labeling with amino acids (SILAC) protocol in A. nidulans}

Stable isotope labeling with amino acids in cell culture (SILAC) is a well-established method for cell cultures or yeast to relatively quantify proteomic changes in different cultures (de Godoy et al., 2006; Ong et al., 2002). Thereby, different isotopically-labeled amino acids are supplemented to the medium of individual cultures. The cells incorporate these amino acid derivatives into their freshly synthesized proteins, which leads to peptide mass differences of basically identical peptides from individual cultures. This allows relative quantitative comparison of proteins deriving from several cell lines and/or culture conditions through LC/MS-MS measurements (Ong et al., 2002). SILAC relies on the amino acids L-lysine and L-arginine (Ong et al., 2003; de Godoy et al., 2006). Mammals cannot synthesize these amino acids and need to take them up from supplied nutrients. Thus, mammalian cell lines rely on the supplementation of these amino acids in the culture medium anyhow.

Fungi are prototroph and synthesize the amino acids L-lysine and L-arginine by themselves through endogenous synthesis pathways (Arst, 1977; Weidner et al., 1997). Strains auxotrophic for L-lysine and L-arginine, respectively, are required to use SILAC for relative quantification of protein abundances. This method is often applied with the unicellular ascomycete $S$. cerevisiae (de Godoy et al., 2006). However, it was not established for the filamentous Aspergillus spp. so far. In the scope of this study, $A$. nidulans L-lysine or L-arginine auxotrophic strains were genetically constructed and the first SILAC experiments with that fungus were conducted.

The biosynthesis pathways of L-lysine and L-arginine in A. nidulans are depicted in Figure 10. L-lysine is synthesized in $A$. nidulans through the a-aminoadipate pathway (Pees, 1967; 
Weidner et al., 1997). The biosynthesis pathway starts with $\alpha$-ketoglutaric acid, which is in several reactions converted to L- $\alpha$-aminoadipic acid. L- $\alpha$-aminoadipic acid is further converted in a three-step process to L-lysine, but it can also be used for penicillin synthesis in $A$. nidulans (Figure 10). The last step in the biosynthesis pathway, which is the conversion of saccharopine to L-lysine, is catalyzed by the saccharopine dehydrogenase encoded by lysA (Weidner et al., 1997). In this study, the lys $A$ gene was deleted to generate an L-lysine auxotrophic $A$. nidulans strain.

The L-arginine biosynthesis is closely linked to the ornithine metabolism (Arst, 1977). L-ornithine is converted to L-citrulline in a three-step process (Figure 10). The argininosuccinate synthase encoded by the $\arg C$ gene catalyzes the conversion from L-citrulline to L-arginosuccinate, which finally gets metabolized to L-arginine. The $\arg C$ gene was here deleted to interrupt the L-arginine biosynthesis in A. nidulans (Figure 10).
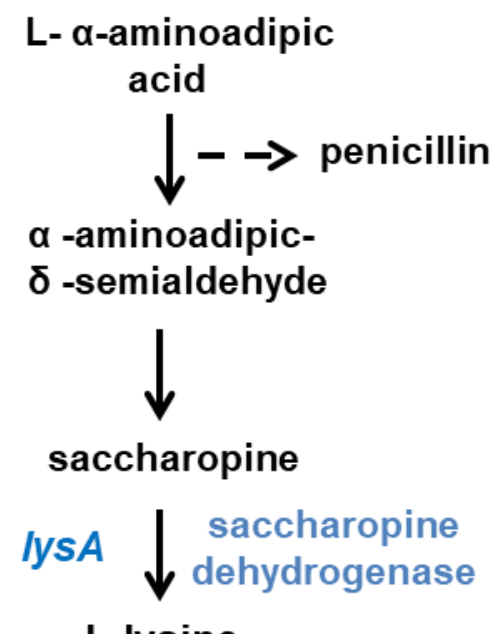

L-lysine
L-ornithine

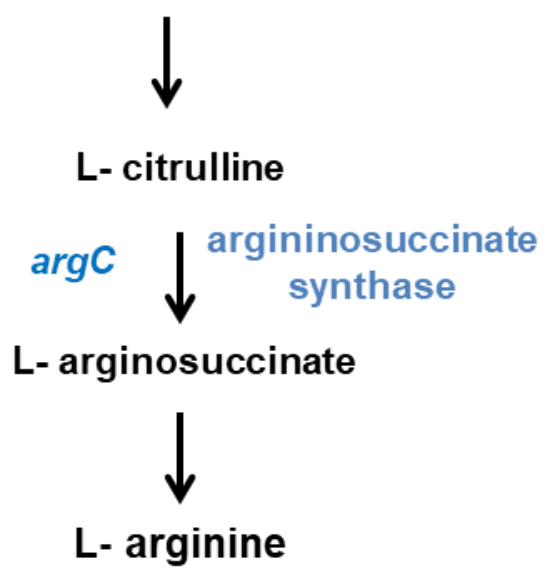

Figure 10: Schematic representation of the L-lysine and L-arginine biosyntheses in A. nidulans.

The biosynthesis of L-lysine is closely linked to penicillin production (left panel). The gene encoding the last enzyme in the biosynthetic pathway, lys $A$, was deleted to generate an L-lysine auxotrophic $A$. nidulans strain (adapted from Weidner et al, 1997). The L-arginine biosynthesis is closely linked to the ornithine metabolism (right panel). An L-arginine auxotroph $A$. nidulans strain was generated by deletion of the $\arg C$ gene, which encodes the argininosuccinate synthase (adapted from Arst, 1977). 


\subsubsection{Generation of L-lysine or L-arginine auxotrophic A. nidulans strains}

SILAC is a well-established method for S. cerevisiae (de Godoy et al., 2006; Gruhler et al., 2005; Schmitt et al., 2017). Strains with a deletion of the LYS1 gene that encodes saccharopine dehydrogenase are generally used for SILAC experiments in yeast (de Godoy et al., 2006). The full ORF of the orthologous $A$. nidulans lys $A$ gene was replaced by a recyclable marker cassette through homologous recombination in the scope of the present study. Only a $100 \mathrm{bp}$ long six site is left at the original lysA gene locus after recycling of the marker cassette (see Chapter 2.4.2). Strains with this gene deletion are not able to grow on minimal medium (MM) without additional L-lysine supplementation. L-lysine auxotrophy can be complemented by reintroducing the lys $A$ gene at the genomic locus (Figure $11 \mathrm{~A}$ ). The $\Delta / y s A$ strain exhibits a wild type like phenotype on medium supplemented with lysine, but grew slightly slower compared to the wild type strain. This was fully complemented by reintroduction of the lys $A$ gene into its original locus (Iys $A^{\text {comp }}$ ).
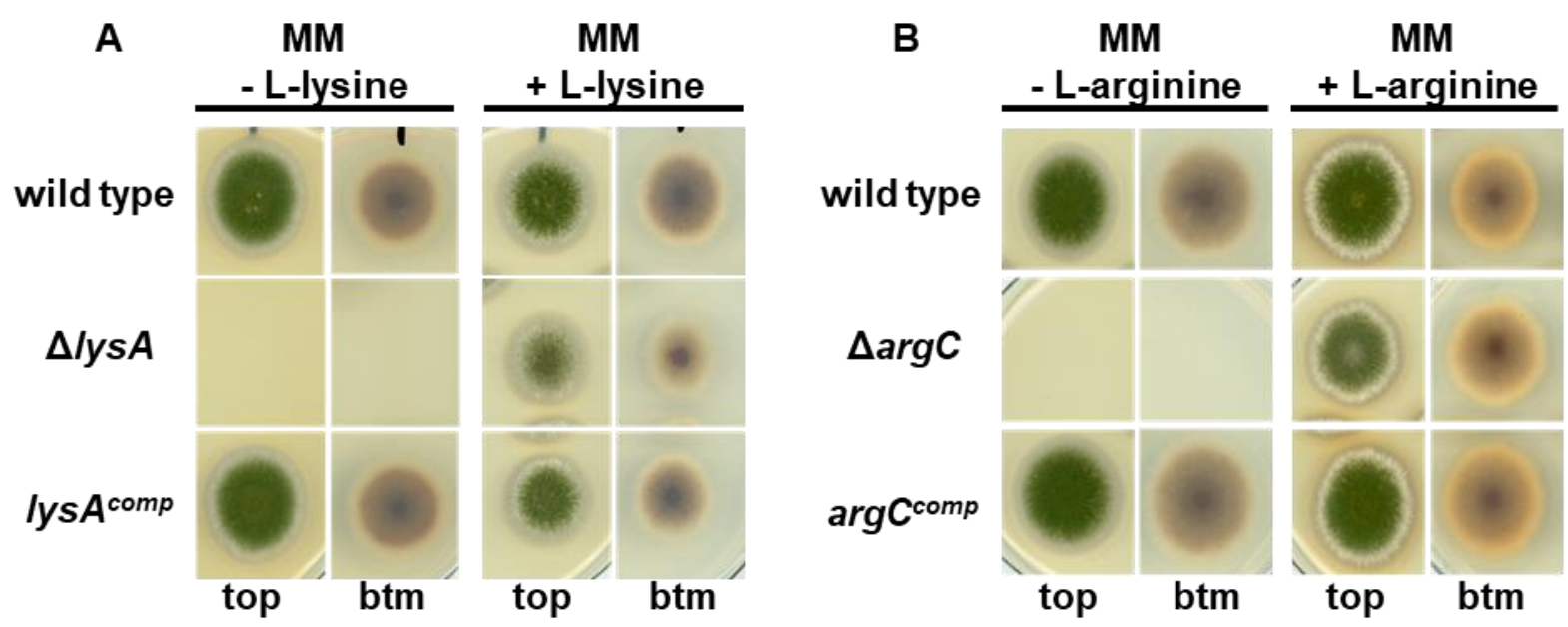

Figure 11: Phenotypes of $A$. nidulans L-lysine and L-arginine auxotrophic strains.

A) Wild type, $\triangle l y S A$ and $l y s A^{\text {comp }}$ were point inoculated with 5,000 spores on agar plates containing minimal medium (MM) (left panel) or MM supplemented with $0.75 \mathrm{mM}$ L-lysine (right panel). In the IysA deletion strain the full ORF was replaced by a recyclable marker cassette (AGB1092). This strain is only able to grow if L-lysine is supplemented into the minimal medium. B) Wild type, $\triangle \arg C$ and $\arg C^{c o m p}$ were point inoculated with 5,000 spores on agar plates containing MM (left panel) or MM supplemented with $10 \mathrm{mM} \mathrm{L-arginine} \mathrm{(right} \mathrm{panel).} \mathrm{Like} \mathrm{for}$ IysA, the $\arg C$ ORF was fully replaced by a recyclable marker cassette (AGB 1154) and is only able to grow when the $\mathrm{MM}$ is additionally supplemented with L-arginine. All plates were incubated for four days at $37^{\circ} \mathrm{C}$ under constant illumination. Addition of the L-lysine or L-arginine to the minimal medium leads to wild type like growth of the L-lysine $(\Delta / y s A)$ or the L-arginine $(\triangle \arg C)$ auxotrophic strains, respectively. Top and bottom (btm) view of the colonies are shown. 
Similarly, the complete $\arg C \mathrm{ORF}$ was replaced by a recyclable marker cassette through homologous recombination and successfully complemented by reintroduction of the $\arg C$ gene at its original gene locus. The deletion strain showed a wild type like phenotype when the minimal medium was additionally supplemented with $10 \mathrm{mM} \mathrm{L-arginine} \mathrm{(Figure} \mathrm{11B).}$

Sample preparation steps for MS-based SILAC experiments include the in-gel tryptic digestion of the extracted proteins with the endopeptidase trypsin. Hydrolysis with trypsin results in peptides with either an L-arginine or a L-lysine as C-terminal residue as trypsin hydrolyzes peptide bonds after these amino acids (Simpson, 2006). A simultaneous labeling with L-lysine and L-arginine would further increase the number of peptides usable for protein quantification after tryptic digestion. Therefore, the generation of a double deletion $\Delta / y s A, \Delta a r g C$ strain would be favorable to enable simultaneous labeling, but the generation of a viable double deletion strain could not be achieved during this study. Therefore, the individual auxotrophic strains for L-lysine or L-arginine can be separately supplemented with the different isotopically-labeled amino acids. All proteins would contain the isotopically-labeled L-lysine or L-arginine variants in the respective strains due to the enforced deficiency of naturally synthesized L-lysine or L-arginine and are distinguishable in LC/MS-MS analyses.

\subsubsection{Validation of $\boldsymbol{A}$. nidulans SILAC strains by incorporation of isotopically-labeled amino acids}

A SILAC amino acid incorporation test was performed to evaluate the efficiency of the uptake and incorporation of the isotopically-labeled L-lysine variants into freshly synthesized proteins by the filamentous fungus. Therefore, two separate cultures of $\Delta / y s A$ strains were grown for $24 \mathrm{~h}$ vegetatively in liquid minimal medium supplemented with different labeled L-lysine isotopes at $37^{\circ} \mathrm{C}$ under agitation. One culture was supplemented with the unlabeled L-lysine (light) and the other one with isotopically-labeled L-lysine variant (Lys8, heavy). Mycelia of the single cultures were harvested by filtration through a Miracloth filter, washed with $0.96 \%(\mathrm{w} / \mathrm{v}) \mathrm{NaCl}$ solution and manually pestled in liquid nitrogen (Figure 12).

The grained mycelium of the two different cultures was mixed in a 1:1 ratio for the following protein extraction step. Additionally, proteins were isolated from mycelia from the separate cultures as well (Figure 12). Extracted proteins were subjected to a denaturing SDS-PAGE. The part of the gel comprising proteins of a molecular weight between 60 and $80 \mathrm{kDa}$ was excised and used for in-gel digestion of proteins with trypsin and subsequent LC/MS-MS analysis. 


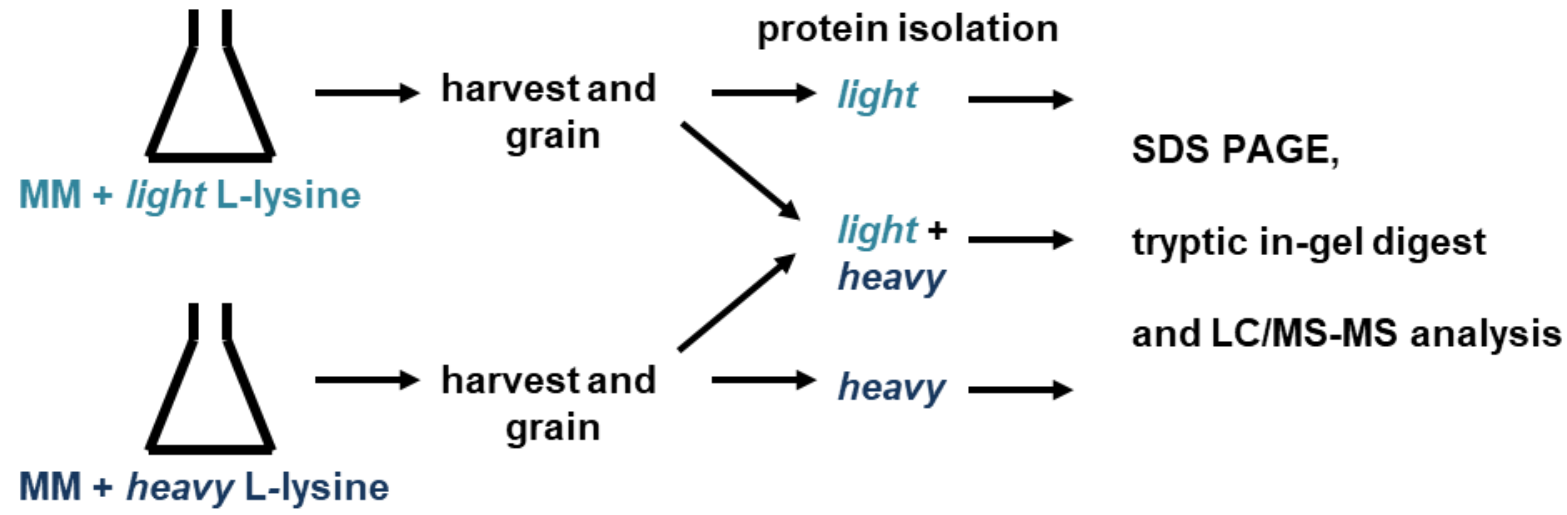

Figure 12: Schematic representation of the workflow for the SILAC amino acid incorporation test.

Two liquid cultures of $\Delta l y s A$ were supplemented with either unlabeled L-lysine (light) or isotopically-labeled L-lysine (heavy) and grown for $24 \mathrm{~h}$ vegetatively at $37^{\circ} \mathrm{C}$. After harvesting of the mycelium of the single cultures through filtration, the mycelium was manually grained. Proteins were isolated from mycelium derived from the different cultures, respectively, and from a 1:1 mixture of mycelia derived from the different cultures. Proteins were separated through SDS-PAGE subjected to tryptic in-gel digestion and subsequent LC/MS-MS analysis.

A similar number of peptides was identified in LC/MS-MS analyses for the individual crude extracts: 4,654 peptides for the culture supplemented with unlabeled light L-lysine, 4,483 peptides for the culture supplemented with the heavy L-lysine variant and 4,634 peptides of the mycelia containing the 1:1 mixture of both cultures (Table 10).

Table 10: Labeling efficiency with SILAC amino acids.

The L-lysine auxotrophic strain $(\Delta / y s A)$ AGB1092 was grown in two separate liquid cultures for $24 \mathrm{~h}$ at $37^{\circ} \mathrm{C}$ under constant agitation. One culture was supplemented with minimal medium (MM) and unlabeled L-lysine (light), whereas the other culture was supplemented with the isotopically-labeled heavy L-lysine variant. Protein crude extracts were prepared from the mycelia deriving from the single cultures and additionally from a 1:1 mixture of mycelia derived from both cultures. Equal amounts of the single protein crude extracts $(40 \mu \mathrm{g})$ were separated through SDS-PAGE and the region in between $60-80 \mathrm{kDa}$ was used for subsequent tryptic digestion and LC/MS-MS analyses. In all samples circa 4,500 peptides have been identified in total. Approximately $60 \%$ of all identified peptides contained at least one L-lysine residue and are therewith putative candidates for quantification. Incorporation of isotopically-labeled (heavy) L-lysine is as efficient as the incorporation of the unlabeled L-lysine counterpart. Data analysis was performed with Proteome Discoverer ${ }^{\text {TM }} 1.4$ (THERMO FISHER SCIENTIFIC).

\begin{tabular}{|l|l|l|l|}
\hline & \multicolumn{3}{|c|}{ Number of peptides } \\
\hline Sample & Total & light L-lysine & heavy L-lysine \\
\hline light & 4,654 & 2,815 & 10 \\
\hline heavy & 4,483 & 2,772 & 2,768 \\
\hline heavy and light & 4,634 & 2,892 & 1,466 \\
\hline
\end{tabular}


Among the identified peptides, approximately 2,800 contained L-lysine residues, which is equal to about $60 \%$ of all peptides. These peptides can be used for the relative quantification of protein abundance. The sample derived from the culture that was supplemented with the heavy L-lysine variant comprised 2,772 L-lysine containing peptides, whereby $99.85 \%$ of these peptides incorporated the heavy lysine isotope (Table 10, Figure 13). In the culture grown in minimal medium supplemented with the light L-lysine variant only ten peptides (less than $0.5 \%$ ) were false positives and wrongly identified as the heavy L-lysine variant. The different L-lysine variants used for supplementation of the culture medium do not have any bias on the fungal protein synthesis. The heavy L-lysine variant is as efficiently incorporated into freshly synthesized proteins as the unlabeled light variant. Combining equal amounts of mycelia from independent cultures supplemented with the different L-lysine variants prior to protein extraction resulted in an equal distribution of peptides containing unlabeled, light (49.31\%) and isotopically-labeled, heavy (50.69 \%) amino acid variants.

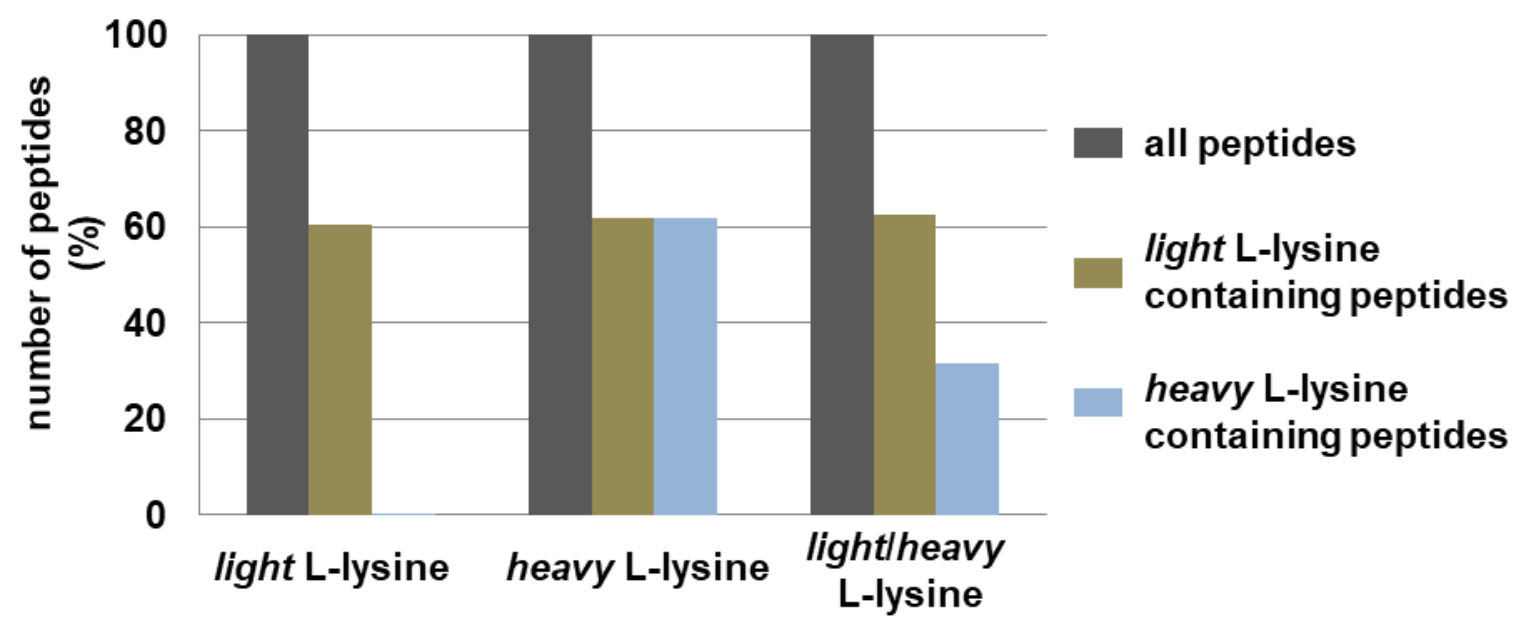

Figure 13: Incorporation efficiency of isotopically-labeled L-lysine variants into fungal proteins during vegetative growth.

$\Delta l y s A$ strain was grown in two liquid cultures containing minimal medium supplemented with light or heavy L-lysine, respectively. Proteins were isolated from the single cultures and from a 1:1 mixture of mycelia derived from both cultures. After separation of proteins by SDS-PAGE, the region between 60 - $80 \mathrm{kDa}$ was used for in-gel tryptic digestion and subsequent LC/MS-MS analyses. Approximately $60 \%$ of all identified peptides contained at least one L-lysine residue. In the culture that was supplemented with the isotopically-labeled L-lysine variant (heavy) the amino acid was incorporated into $99.85 \%$ of all peptides. The combination of mycelia of differentially labeled cultures (light/heavy L-lysine) revealed equal distribution of peptides containing the unlabeled (light) or the isotopically-labeled (heavy) L-lysine variant. 
A. nidulans is able to efficiently take up different isotopically-labeled variants of L-lysines, which is a prerequisite for SILAC experiments, and can use them for protein synthesis. Furthermore, the combination of equal amounts of grained mycelia prior to protein extraction leads to an equal distribution of peptides containing the different L-lysine variants. The efficient incorporation of the different amino acids indicated that the SILAC approach can be used for relative quantification of proteins in the filamentous fungus $A$. nidulans.

\subsubsection{Construction of SILAC strains with an impaired COP9 signalosome}

The COP9 signalosome is a conserved eight-subunit protein complex, which regulates the ubiquitin-proteasome pathway through deneddylation of CRLs (Cope et al., 2002; Wei and Deng, 2003). It removes the posttranslational modification Nedd8 from cullins and renders the ubiquitination machinery temporary inactive (Beckmann et al., 2015). The incorporation of the deneddylase subunit CsnE confers the catalytic activity to the complex (Beckmann et al., 2015; Lingaraju et al., 2014).

The effect of a dysfunctional COP9 signalosome lacking the catalytically active CsnE subunit on the fungal protein turn-over during vegetative growth in liquid culture conditions was investigated. csnE deletion strains develop similar vegetative hyphae as wild type strains (Nahlik et al., 2010). Here, it was examined how protein abundances are changing during this culture conditions and if protein levels are influenced by a dysfunctional COP9 signalosome. The csnE gene was deleted in the lysine auxotrophic strain (Figure 14). A complementation strain $\left(c s n E^{c o m p}\right)$ was included as reference into the analysis to assign the observed regulations in protein abundances specifically to the csnE gene deletion. The $c s n E^{c o m p}$ complements the $c s n E$ deletion phenotype (Figure 14). A functional COP9 signalosome is required for accurate asexual or sexual development. The $\Delta c s n E$ mutant strain is impaired in sexual fruiting body formation and shows altered secondary metabolism (Beckmann et al., 2015; Busch et al., 2007; Nahlik et al., 2010). Similar phenotypes are also observed in the $\Delta / y s A$ background strain (Figure 14).

Therefore, these strains are suitable for the following SILAC experiment. An additional functional gene fusion for a GFP-tagged CsnD subunit was introduced into the original genomic locus of csnD (Beckmann et al., 2015). This allows future GFP pull down experiments to analyze COP9 signalosome interaction partners or posttranslational modifications of CSN subunits quantitatively. The $\Delta l y s A$, gfp::csnD strain served as control for the following SILAC experiment. 


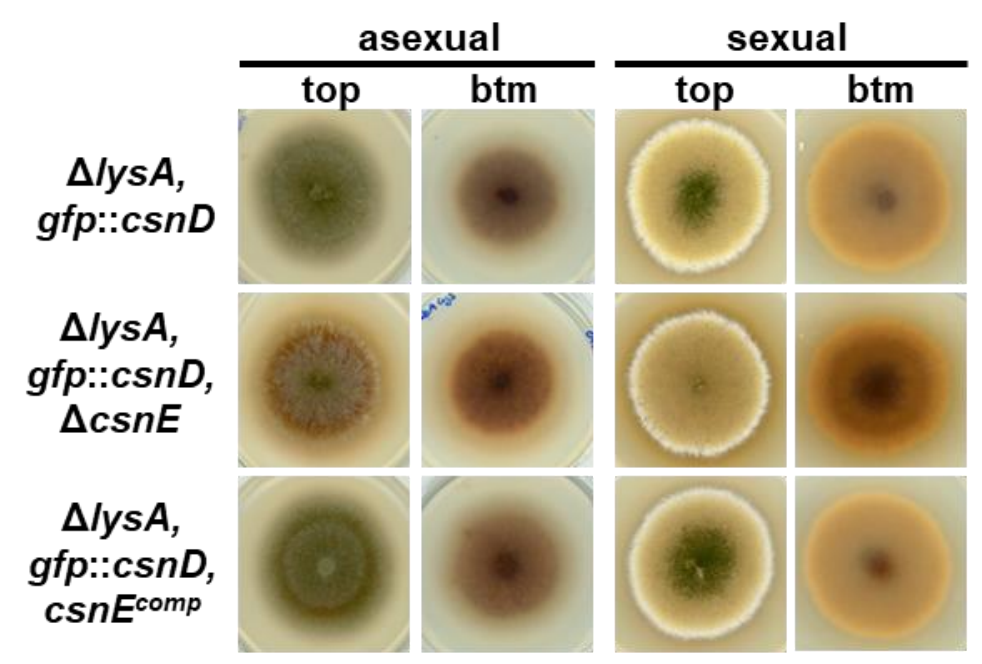

Figure 14: CsnE is required for the development of asexual spores, cleistothecia and regulation of secondary metabolism.

L-lysine auxotrophic strains carrying gfp::csnD fusion ( $\Delta / y s A, g f p:: c s n D)$ and $\Delta \operatorname{csn} E(\Delta / y s A$, gfp::csnD, $\Delta c s n E)$ or $\operatorname{csn} E$ complementation $\left(\Delta / y s A, g f p:: c s n D, c s n E^{c o m p}\right)$ were point-inoculated using 5,000 spores on solid agar plates containing minimal medium supplemented with $0.75 \mathrm{mM}$ light L-lysine. Agar plates were incubated for four days at $37^{\circ} \mathrm{C}$ in light to induce asexual development and seven days at $37^{\circ} \mathrm{C}$ in darkness and under elevated carbon dioxide pressure to induce sexual development. Top and bottom (btm) views of the colonies are shown.

The $\Delta l y s A, g f p:: c s n D, \Delta c s n E$ strain was used to analyze the effect of a functional COPg signalosome on the proteome of vegetatively grown cultures of $A$. nidulans and this was compared to the background strain $\Delta l y s A, g f p:: c s n D$. The complementation strain $\Delta l y s A$, gfp::csnD, $\operatorname{csn} E^{c o m p}$ was included into the analysis to ensure that observed changes in the protein abundances can be attributed to the lack of CsnE.

\subsubsection{The COP9 signalosome changes more than $10 \%$ of the fungal proteome during vegetative growth}

The SILAC experiment was performed to analyze changes of protein abundances in the absence of the catalytically active subunit CsnE of the COP9 signalosome. Therefore, the three SILAC strains: $\Delta l y s A(\Delta l y s A, g f p:: c s n D) ; \Delta \operatorname{csn} E(\Delta l y s A$, gfp::csnD, $\Delta c s n E)$ and $c s n E^{c o m p}(\Delta l y s A$, gfp::csnD, $c s n E^{c o m p}$ ) were grown for $24 \mathrm{~h}$ at $37^{\circ} \mathrm{C}$ in liquid culture to induce vegetative development. All strains were grown in minimal medium supplemented with different isotopicallylabeled L-lysine variants. The culture with $\Delta / y s A$, which served as wild type like control, was supplemented with the heavy L-lysine variant $\left({ }^{13} \mathrm{C}^{15} \mathrm{~N}\right.$ labeled lysine $\left.{ }_{*} \mathrm{HCl}\right)$. The deletion strain $\Delta c s n E$ was fed with the light L-lysine variant. Furthermore, the $c s n E^{c o m p}$ was included as 
additional control and supplemented with the medium L-lysine variant (D4 labeled lysine $2 \mathrm{HCl}$ ). The different strains incorporated the different isoptopically-labeled L-lysine variants into their proteins and in subsequent LC/MS-MS analyses identical proteins resulting from the different strains are distinguishable by their mass.

After harvesting of the mycelium through filtration and washing with $0.96 \%(\mathrm{w} / \mathrm{v}) \mathrm{NaCl}$ solution, the mycelium of all strains was grained separately. Subsequently, equal amount of frozen mycelia $(0.02 \mathrm{~g})$ were combined for protein crude extract preparation. The extracted proteins were subjected to SDS-PAGE. Afterwards, the whole lanes of the SDS-gel were sliced for in-gel digestion of the proteins with trypsin to generate peptides. For the enzymatic digestion, the lane of the SDS-gel was divided into ten equally sized pieces to achieve good protein coverage with high number of peptides in subsequent LC/MS-MS analyses. The SILAC experiment was conducted in three biological replicates.

The SILAC ratios used for further downstream analyses were generated and normalized by the MaxQuant program based on the peak intensity in the MS1 spectra. Thereby, peak intensities of the peptides were compared in $\triangle c s n E$ and $\triangle / y s A$ as well as $\triangle c s n E$ and $c s n E^{\text {comp }}$ and SILAC ratios were calculated by the MaxQuant program, respectively. Thereby, the value of the respective control strain $\Delta / y s A$ or $c s n E^{c o m p}$ is in the denominator. SILAC ratios between $c s n E^{c o m p}$ and $\Delta l y s A$ background strain were also compared, which should not change significantly in protein abundances that are influenced by CsnE. The SILAC ratios represent the fold change of the proteins and were logarithmized with $\log _{2}$ for downstream analyses with Perseus. Therefore, a value of 0 indicates no difference in protein abundance in the different cultures. High numbers for SILAC ratios indicate increased protein abundance, whereas low values indicate decreased protein abundance in $\Delta c s n E$ compared to $c s n E^{c o m p}$ or $\Delta / y s A$. In protein abundances between $c s n E^{c o m p}$ and the background strain $\triangle l y s A$ no change was expected. Therefore, only proteins with a $\log _{2}$ SILAC ratio between $-0.5 \leq 0 \leq 0.5$ in $c s n E^{\text {comp }} / \Delta / y s A$ were selected for further analyses. Proteins, which show already changes in protein abundance in this two control strains were defined as false positives and not considered for further data analyses.

After this filtering step, 745 proteins were left. Mean SILAC ratios were calculated from the three biological replicates. Minor increased protein abundance was defined for proteins with a mean $\log _{2}$ SILAC ratio of $0.5 \leq x \leq 0.8$. Thereby, 18 proteins showed minor increased abundance in $\triangle c s n E$ compared to $c s n E^{c o m p}$ or $\Delta l y s A$ background (Figure 15 , light green squares). This equals $2.4 \%$ of the identified proteins used for the analysis. Higher $\log _{2}$ fold changes $(>0.8)$ were observed for 22 more proteins (Figure 15, dark green squares), which equals $3 \%$. In total, 
among the 745 proteins considered as suitable for further downstream analysis, $5.4 \%$ of the proteins showed increased abundance in $\triangle c s n E$.

The change of the threshold about $\log _{2} 0.3$ nearly duplicates the list of candidates, which abundances are positively influenced by CsnE. Applying the same threshold for the proteins that show decreased abundance in $\Delta c s n E$ compared to $c s n E^{c o m p}$ and $\Delta / y s A$ revealed a number of 59 proteins, which equals $7.8 \%$ of all proteins used for the downstream analyses. Thereby, a $\log _{2}$ ratio of $-0.5 \leq x \leq-0.8$ was calculated for $5.2 \%$ (39 proteins) and are considered to have a minor decrease in their protein abundance (Figure 15, light blue squares) and $2.6 \%$ (20 proteins) show a higher decrease in their protein abundance (Figure 15, dark blue squares).

Taken together, the SILAC experiment showed that $13.2 \%$ of the proteins equal to 99 proteins analyzed during this study are influenced in their abundance by the function of the COP9 signalosome. Thereby, the protein abundance of $5.4 \%$ of the proteins is reduced by a functional COP9 signalosome, whereas $7.8 \%$ of the proteins are increased in their abundance. Deletion of the catalytic subunit $\operatorname{csn} E$ and therewith the interference with the $\mathrm{CRL}$ catalyzed ubiquitination cycles influences fungal proteins already during vegetative growth conditions.

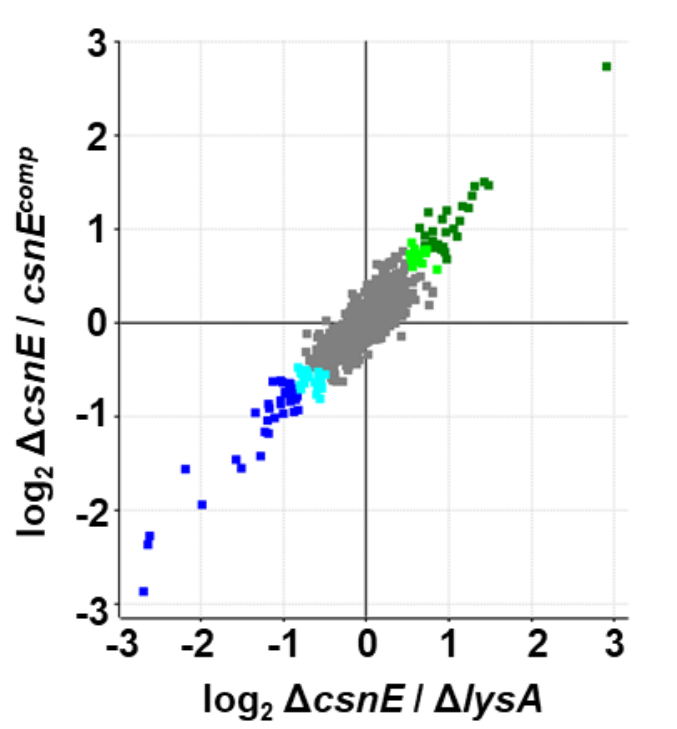

Figure 15: Scatter plot of proteins identified in SILAC experiment.

Each square in the scatter plot represents one identified protein. $86.8 \%$ of these proteins were not changed in their abundance in the absence of CsnE (depicted in grey). Light green squares represent proteins with a mean $\log _{2}$ SILAC ratio of three biological replicates of $0.5 \leq x \leq 0.8$, whereas dark green squares have $\log _{2}$ SILAC ratios of higher than 0.8 . The abundances of these proteins are increased in $\Delta c s n E$ compared to $c s n E^{c o m p}$ and $\Delta l y s A$ background strain. Blue squares indicate proteins that abundance is decreased in the absence of csnE. Light blue squares have $\log _{2}$ SILAC ratios between $-0.5 \leq x \leq-0.8$, whereas dark blue squares show $\log _{2}$ ratios lower than -0.8 . 


\subsubsection{CsnE inhibits increased protein amounts of amino acid and vitamin metabolism, oxidoreductases and development related proteins during vegetative fungal growth}

The abundance of 40 proteins is increased in the absence of CsnE. These proteins are listed in Table 11. The mean $\log _{2}$ of the SILAC ratios from three biological replicates represent the fold changes of the single proteins. The fold change shown here represents the ratio of the protein amount in $\Delta c s n E$ compared to the background strain $\Delta l y s A$. These fold changes are similar to the ones observed in $\triangle c s n E$ compared to $c s n E^{c o m p}$, which are shown in Table S1. There are also the mean $\log _{2}$ SILAC ratios of the control $c s n E^{\text {comp }} / \Delta / y s A$ listed (Table S1).

Table 11: Proteins with increased abundance during vegetative growth of $\boldsymbol{A}$. nidulans in $\Delta c s n E$.

The $\log _{2}$ SILAC ratios (fold changes) of proteins in $\Delta c s n E$ compared to $\Delta l y s A$ background strain with a wild type like csnE gene locus ( $c s n E$ ) are given. High values indicate increased protein abundances in the deletion of csnE. Thereby, the intensity of the green color decreases with decreasing protein abundance. The description of identified proteins derived from information given from AspGD and FungiDB (Cerqueira et al., 2014; Stajich et al., 2012). The bold line defined the threshold between minor increased protein abundance and stronger increase in protein abundance. This threshold equals the SILAC ratio described in Chapter 3.1.2, where protein with a $\log _{2}$ SILAC ratio between $0.5 \leq x \leq 0.8$ were defined as minor increased, and proteins with a SILAC ratio $\geq 0.8$ as increased in their abundance.

\begin{tabular}{|c|c|c|}
\hline $\begin{array}{c}\log _{2} \\
\text { Fold Change } \\
\Delta \operatorname{csn} E / \Delta / y s A\end{array}$ & $\begin{array}{l}\text { Systematic } \\
\text { Name }\end{array}$ & Description \\
\hline 2.90 & ANIA_05449 & $\begin{array}{l}\text { Domains with sulfuric ester hydrolase activity and role in } \\
\text { metabolic process }\end{array}$ \\
\hline 1.47 & ANIA_01805 & CanB, putative carbonic anhydrase \\
\hline 1.42 & ANIA_06338 & $\begin{array}{l}\text { Putative aromatic-amino-acid transaminase with a predicted } \\
\text { role in aromatic amino acid biosynthesis }\end{array}$ \\
\hline 1.31 & ANIA_07914 & $\begin{array}{l}\text { OrsE; member of the F9775 secondary metabolite gene } \\
\text { cluster }\end{array}$ \\
\hline 1.28 & ANIA_00495 & $\begin{array}{l}\text { Predicted amino acid binding, formyltetrahydrofolate } \\
\text { deformylase activity }\end{array}$ \\
\hline 1.23 & ANIA_01621 & Putative GNAT-type acetyltransferase \\
\hline 1.16 & ANIA_04323 & $\begin{array}{l}\text { Putative branched chain amino acid aminotransferase with a } \\
\text { predicted role in valine, leucine, and isoleucine metabolism }\end{array}$ \\
\hline 1.13 & ANIA_10223 & Putative 1-Cys peroxiredoxin \\
\hline 1.10 & ANIA_02284 & $\begin{array}{l}\text { HemA, putative } 5 \text {-aminolevulinic acid synthase, involved in } \\
\text { heme synthesis }\end{array}$ \\
\hline 1.05 & ANIA_01752 & $\begin{array}{l}\text { Putative sulfite reductase with a predicted role in sulfur } \\
\text { metabolism }\end{array}$ \\
\hline 0.97 & ANIA_04430 & $\begin{array}{l}\text { Small subunit of acetolactate synthase involved in branched- } \\
\text { chain amino acid biosynthesis under hypoxic conditions }\end{array}$ \\
\hline 0.97 & ANIA_00840 & $\begin{array}{l}\text { Putative alpha-isopropylmalate synthase with a predicted role } \\
\text { in valine, leucine, and isoleucine metabolism }\end{array}$ \\
\hline
\end{tabular}


Table 11: continued.

\begin{tabular}{|c|c|c|}
\hline $\begin{array}{c}\log _{2} \\
\text { Fold Change } \\
\Delta \operatorname{csn} E / \Delta / y s A\end{array}$ & $\begin{array}{l}\text { Systematic } \\
\text { Name }\end{array}$ & Description \\
\hline 0.96 & ANIA_07600 & $\begin{array}{l}\text { Ortholog(s) have role in hydrogen sulfide biosynthetic } \\
\text { process, sulfate assimilation, sulfur amino acid biosynthetic }\end{array}$ \\
\hline 0.95 & ANIA_03169 & $\begin{array}{l}\text { Putative ribose-phosphate pyrophosphokinase with a } \\
\text { predicted role in histidine metabolism }\end{array}$ \\
\hline 0.92 & ANIA_08605 & $\begin{array}{l}\text { Cyp1, putative peptidyl-prolyl cis-trans isomerase (PPlase), } \\
\text { involved in ascospore formation }\end{array}$ \\
\hline 0.92 & ANIA_05616 & Putative kynurenine aminotransferase \\
\hline 0.90 & ANIA_02999 & Isocitrate dehydrogenase (NADP+), putative \\
\hline 0.86 & ANIA_03031 & $\begin{array}{l}\text { Putative threonine synthase with a predicted role in glycine, } \\
\text { serine, and threonine metabolism }\end{array}$ \\
\hline 0.85 & ANIA_00158 & $\begin{array}{l}\text { Ortholog(s) have } 2 \text {-alkenal reductase [NAD(P)] activity, } \\
\text { AU-rich element binding }\end{array}$ \\
\hline 0.82 & ANIA_07725 & PyroA, protein required for biosynthesis of pyridoxine \\
\hline 0.80 & ANIA_10298 & 3-phosphoserine aminotransferase \\
\hline 0.80 & ANIA_05181 & NudC, protein involved in nuclear migration \\
\hline 0.75 & ANIA_07708 & $\begin{array}{l}\text { Ortholog(s) have alditol:NADP+1-oxidoreductase activity, } \\
\text { role in D-xylose, arabinose catabolic process }\end{array}$ \\
\hline 0.72 & ANIA_02981 & $\begin{array}{l}\text { Putative glucose 6-phosphate 1-dehydrogenase with a } \\
\text { predicted role in the pentose-phosphate shunt }\end{array}$ \\
\hline 0.72 & ANIA_01990 & Putative homocitrate synthase, role in pyruvate metabolism \\
\hline 0.71 & ANIA_05820 & $\begin{array}{l}\text { Cystathionine beta-synthase, an enzyme involved in } \\
\text { methionine, S-adenosylmethionine, and cysteine biosynthesis }\end{array}$ \\
\hline 0.70 & ANIA_04401 & Putative asparagine synthase, role in asparagine metabolism \\
\hline 0.69 & ANIA_07567 & $\begin{array}{l}\text { Ortholog(s) have RNA polymerase II activating transcription } \\
\text { factor binding, glutathione disulfide oxidoreductase activity }\end{array}$ \\
\hline 0.67 & ANIA_06655 & Putative glucose 1-dehydrogenase \\
\hline 0.65 & ANIA_08277 & $\begin{array}{l}\text { Putative bifunctional enzyme with a predicted role in } \\
\text { methionine metabolism }\end{array}$ \\
\hline 0.63 & ANIA_04462 & Putative pyruvate carboxylase or glutathione synthase \\
\hline 0.61 & ANIA_03712 & Uncharacterized \\
\hline 0.59 & ANIA_10219 & Uncharacterized \\
\hline 0.59 & ANIA_04793 & $\begin{array}{l}\text { Putative aspartate semialdehyde dehydrogenase with a } \\
\text { predicted role in glycine, serine, and threonine metabolism }\end{array}$ \\
\hline 0.58 & ANIA_00893 & $\begin{array}{l}\text { Putative adenylosuccinate synthase with a predicted role in } \\
\text { purine metabolism }\end{array}$ \\
\hline 0.57 & ANIA_05904 & $\begin{array}{l}\text { Putative beta-ketoacyl-[acyl-carrier-protein] synthase with a } \\
\text { predicted role in cytosolic fatty acid formation }\end{array}$ \\
\hline 0.55 & ANIA_01662 & Uncharacterized \\
\hline 0.54 & ANIA_00354 & $\begin{array}{l}\text { AroG, putative 3-deoxy-D-arabino-heptulosonate 7- } \\
\text { phosphate synthase, role in aromatic amino acid biosynthesis }\end{array}$ \\
\hline 0.53 & ANIA_02976 & Uncharacterized \\
\hline 0.53 & ANIA_05999 & Carbamoyl-phosphate synthase, arginine biosynthesis \\
\hline
\end{tabular}


14 of the 40 upregulated proteins in $\Delta c s n E$ compared to the control strains were classified to amino acid metabolism (Figure 16). Furthermore, eight proteins were assigned as proteins involved in electron ( $\left.e^{-}\right)$transfer or vitamin biosynthesis. Minor reduced protein abundance was detected for AroG in $\Delta c s n E$, which is one of two 3-deoxy-D-arabino-heptulosonate-7-phosphate (DAHP) producing enzymes in A. nidulans (Hartmann et al., 2001). AroG is involved in the aromatic amino acids biosynthesis and inhibited by the end product of the regulated synthesis pathway, phenylalanine (Hartmann et al., 2001). OrsE, which is encoded by a member of the F9975 secondary metabolite gene cluster, is the only protein here that was classified to secondary metabolism (Table 11). The production of orsellinic acid and its derivates in strains carrying csn mutations was already described previously (Beckmann et al., 2015; Busch et al., 2007).

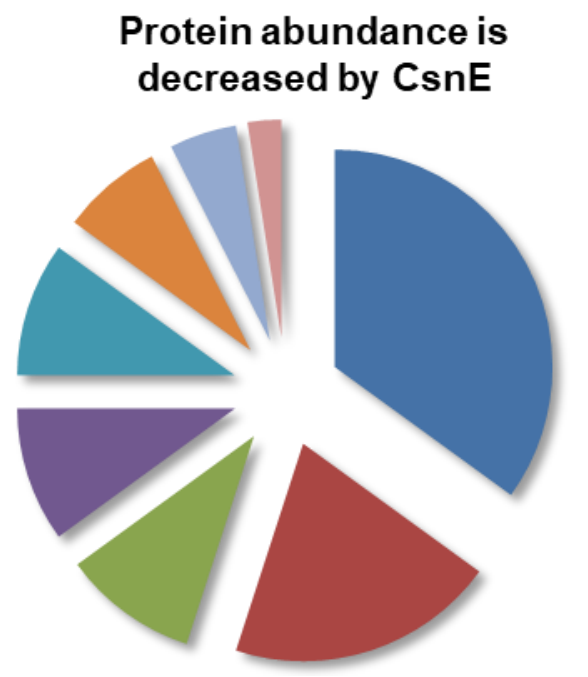

Amino acid metabolism: 14

etransfer, cofactors, vitamin biosynthesis: 8

Development: 4

Carbohydrate metabolism: 4

\section{Others: 3}

Stress: 2

Secondary metabolism: 1

Uncharacterized: 4

Figure 16: Classification of proteins, which abundances are decreased by CsnE.

Proteins listed in Table 11 were assigned to different categories. The number behind the category indicates the number of proteins assigned to this class. One fourth of all proteins decreased in the abundance by the presence of CsnE were classified to amino acid metabolism.

Furthermore, two proteins associated to stress response and four proteins related to fungal development were increased in their abundance in $\Delta c s n E$. Among them is NudC, a protein involved in nuclear movement and cell wall synthesis, Cyp1, which is involved in the formation of ascospores deriving from the sexual reproduction cycle, and CanB, which is involved in the conidiation process (Cerqueira et al., 2014; Chiu et al., 1997; Han et al., 2010).

In total, $65 \%$ of the proteins, which show increased abundance in $\Delta c s n E$ are associated to primary metabolism (amino acid biosynthesis; $\mathrm{e}^{-}$transfer, cofactors, vitamin biosynthesis; 
aminotransferases; carbohydrate metabolism). This might indicate a defect or retardation of nutrient assimilation in the csn mutant strain. The COP9 signalosome controls indirectly the supply of amino acids in the cell by deneddylating the cullin scaffold protein of CRLs. Specifically, proteins involved in amino acid metabolism are increased in their abundance in csnE deficient cells, whereas a functional CsnE keeps these protein levels low.

\subsubsection{An intact COP9 signalosome promotes increased protein amounts of septins and cytoskeleton associated proteins during $A$. nidulans vegetative growth}

Proteins, which abundances are increased in $\Delta c s n E$ compared to $\Delta / y s A$ and $c s n E$ complementation strain are listed in Table 12. Thereby, only the fold change of proteins in $\triangle c s n E$ compared to the $\Delta / y s A$ background strain are shown. The fold changes are in a similar magnitude if they are compared to the $c s n E^{c o m p}$ strain. These as well as the fold changes for the control $c s n E^{\text {comp }} / \Delta / y s A$ are listed in Supplementary Table S2.

A majority of these proteins was categorized to fungal development (Figure 17). Among these 15 proteins, four septins were identified. In the absence of a functional COP9 signalosome, septin proteins are reduced in their protein abundance. Furthermore, three proteins namely ANIA_08547, ANIA_06048, ANIA_03636 and Nmt1 are involved in hyphal growth or early stages of conidiophore development, whereas ANIA_08547 has the highest $\log _{2}$ fold change.

Table 12: Proteins with decreased abundance during vegetative growth in a $\Delta c s n E$ strain. $\log _{2}$ SILAC ratios of $\Delta c s n E / \Delta l y s A$ are given in this table. Low values indicate downregulation of protein abundances in the deletion of csnE compared to the wild type like background strain. The intensity of the blue color displays the level of change in protein abundance. The description of identified proteins derived from information given from AspGD and FungiDB (Cerqueira et al., 2014; Stajich et al., 2012). SILAC ratios marked with * could not be exclusively attributed to one protein. The bold line indicates the threshold between minor decreased protein abundance and a stronger decrease in protein abundance. This threshold equals the SILAC ratio described in Chapter 3.1.2, where protein with a $\log _{2}$ SILAC ratio between $-0.5 \leq x \leq-0.8$ were defined as minor decreased, and proteins with a $\log _{2}$ SILAC ratio lower than -0.8 as decreased in their abundance.

\begin{tabular}{|c|c|c|}
\hline $\begin{array}{c}\log _{2} \\
\text { Fold Change } \\
\Delta c s n E / \Delta l y s A\end{array}$ & $\begin{array}{l}\text { Systematic } \\
\text { Name }\end{array}$ & Description \\
\hline-2.69 & ANIA_08547 & $\begin{array}{l}\text { Putative glucose-methanol-choline (GMC) oxidoreductase, } \\
\text { required for early stages of conidiophore development }\end{array}$ \\
\hline-2.64 & ANIA_03344 & Putative GNAT-type acetyltransferase \\
\hline-2.62 & ANIA_10296 & Ortholog(s) have fumarate reductase (NADH) activity \\
\hline-2.19 & ANIA_06058 & $\begin{array}{l}\text { DUF833 domain-containing protein, orthologs have function } \\
\text { in filamentous growth }\end{array}$ \\
\hline
\end{tabular}


Table 12: continued.

\begin{tabular}{|c|c|c|}
\hline $\begin{array}{c}\log _{2} \\
\text { Fold Change } \\
\Delta \operatorname{csn} E / \Delta / y s A\end{array}$ & $\begin{array}{l}\text { Systematic } \\
\text { Name }\end{array}$ & Description \\
\hline-1.99 & ANIA_03524 & Ngn27, acetyltransferase, galactonate metabolism \\
\hline-1.58 & ANIA_03674 & Domains with predicted phospholipid binding activity \\
\hline-1.52 & ANIA_05823 & SidA, L-ornithine N5 monooxygenase \\
\hline-1.35 & ANIA_00858 & Hsp104, heat shock \\
\hline-1.28 & ANIA_09180 & Putative transketolase \\
\hline-1.23 & ANIA_07062 & Uncharacterized \\
\hline-1.19 & ANIA_08009 & Nmt1, thiamine biosynthesis process, hyphal growth \\
\hline-1.18 & ANIA_09148 & GalF, putative UTP-glucose-1-phosphate uridylyltransferase \\
\hline-1.18 & ANIA_08145 & Uncharacterized, putative AN8142 cluster \\
\hline-1.17 & ANIA_04727 & UgeA, UDP-glucose 4-epimerase, galactose metabolism \\
\hline-1.13 & ANIA_03012 & $\begin{array}{l}\text { KapL, role in protein export from nucleus, tRNA re-export } \\
\text { from nucleus and cytosol, nuclear envelope localization }\end{array}$ \\
\hline-1.11 & ANIA_04923 & $\begin{array}{l}\text { Putative 3-hydroxy-3-methylglutaryl coenzyme A synthase } \\
\text { with a predicted role in sterol metabolism }\end{array}$ \\
\hline-1.04 & ANIA_03906 & Uncharacterized \\
\hline-1.04 & ANIA_03223 & $\begin{array}{l}\text { PfkA, putative 6-phosphofructokinase with a predicted role } \\
\text { in gluconeogenesis and glycolysis }\end{array}$ \\
\hline-1.03 & ANIA_01911 & Putative mannose-1-phosphate guanyltransferase \\
\hline$-1.02^{*}$ & $\begin{array}{l}\text { ANIA_05082; } \\
\text { ANIA_10627; } \\
\text { tubB }\end{array}$ & $\begin{array}{l}\text { GTP binding (cytoskelet); } \\
\text { GTP binding (cytoskelet); } \\
\text { GTP binding (cytoskelet) }\end{array}$ \\
\hline-1.01 & ANIA_04908 & Putative eukaryotic translation initiation factor subunit \\
\hline-0.99 & ANIA_10709 & $\begin{array}{l}\text { GfaA, putative glutamine-fructose-6-phosphate } \\
\text { transaminase }\end{array}$ \\
\hline-0.93 & ANIA_08182 & AspC, septin \\
\hline-0.93 & ANIA_01394 & AspD, putative septin \\
\hline-0.93 & ANIA_05586 & $\begin{array}{l}\text { Putative mannose-1-phosphate guanylyltransferase with a } \\
\text { predicted role in mannose, fructose,sorbitol metabolism }\end{array}$ \\
\hline-0.92 & ANIA_06004 & Protein with an RNA recognition motif, asperthecin cluster \\
\hline-0.92 & ANIA_01158 & $\begin{array}{l}\text { Ortholog(s) have mRNA 3'-UTR, mRNA 5'-UTR binding, } \\
\text { translation repressor activity, nucleic acid binding activity }\end{array}$ \\
\hline-0.92 & ANIA_02532 & $\begin{array}{l}\text { Ortholog(s) have copper ion binding, primary amine oxidase } \\
\text { activity }\end{array}$ \\
\hline-0.88 & ANIA_08815 & Putative isoflavone reductase family protein \\
\hline-0.87 & ANIA_07262 & Uncharacterized \\
\hline-0.86 & ANIA_03112 & $\begin{array}{l}\text { UgmA, UDP-galactopyranose mutase, enzyme in } \\
\text { galactofuranose biosynthesis, cell wall }\end{array}$ \\
\hline-0.84 & ANIA_06688 & AspB, putative septin B \\
\hline-0.84 & ANIA_04042 & Putative C-22 sterol desaturase, sterol metabolism \\
\hline-0.83 & ANIA_03829 & $\begin{array}{l}\text { Putative succinate-semialdehyde dehydrogenase [NAD(P)+] } \\
\text { with a predicted role in 4-aminobutyrate (GABA) shunt }\end{array}$ \\
\hline
\end{tabular}


Table 12: continued.

\begin{tabular}{|c|c|c|}
\hline 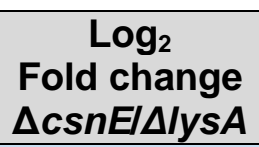 & $\begin{array}{c}\text { Systematic } \\
\text { Name }\end{array}$ & Description \\
\hline-0.83 & ANIA_04591 & $\begin{array}{l}\text { Ortholog(s) have phosphopentomutase activity, role in } \\
\text { guanosine catabolic process }\end{array}$ \\
\hline-0.81 & ANIA_05895 & GdiA, putative Rab GDP-dissociation inhibitor \\
\hline-0.80 & ANIA_04463 & Uncharacterized \\
\hline-0.80 & ANIA_03636 & $\begin{array}{l}\text { Ortholog(s) have role in cellular response to biotic stimulus, } \\
\text { cellular response to starvation and filamentous growth }\end{array}$ \\
\hline-0.80 & ANIA_02925 & $\begin{array}{l}\text { PexF, putative peroxisomal import protein (peroxin) with a } \\
\text { role in fatty acid utilization }\end{array}$ \\
\hline-0.79 & ANIA_08233 & Ortholog(s) have phosphatidylinositol transporter activity \\
\hline-0.79 & ANIA_06341 & Similarity to $S$. cerevisiae Crn1p (cytoskeletal component) \\
\hline-0.78 & ANIA_06266 & Uncharacterized \\
\hline-0.77 & ANIA_04667 & AspA, septin \\
\hline-0.76 & ANIA_02756 & SlaB, predicted actin binding protein \\
\hline-0.75 & ANIA_10614 & $\begin{array}{l}\text { Ortholog(s) have G-quadruplex DNA binding, ribosome } \\
\text { binding, telomeric DNA binding, triplex DNA binding activity }\end{array}$ \\
\hline-0.74 & ANIA_08012 & $\begin{array}{l}\text { Putative bifunctional enzyme with a predicted role in sterol } \\
\text { metabolism }\end{array}$ \\
\hline-0.73 & ANIA_03026 & $\begin{array}{l}\text { CopA, alpha-COP coatamer-related protein involved in the } \\
\text { establishment and maintenance of polarized growth }\end{array}$ \\
\hline-0.71 & ANIA_09094 & $\begin{array}{l}\text { UngA, putative UDP-N-acetylglucosamine } \\
\text { pyrophosphorylase with role in chitin biosynthesis }\end{array}$ \\
\hline-0.70 & ANIA_02867 & $\begin{array}{l}\text { PgmB, putative phosphoglucomutase with a predicted role } \\
\text { in carbohydrate metabolism }\end{array}$ \\
\hline-0.63 & ANIA_07146 & $\begin{array}{l}\text { Putative S-adenosyl-methionine delta-24-sterol-C- } \\
\text { methyltransferase with a predicted role in sterol metabolism }\end{array}$ \\
\hline-0.61 & ANIA_01810 & $\begin{array}{l}\text { OtaA, ornithine transaminase, involved in utilization of } \\
\text { arginine as a proline source }\end{array}$ \\
\hline-0.59 & ANIA_00327 & $\begin{array}{l}\text { Ortholog(s) have ATP-dependent 3'-5' DNA helicase activity, } \\
\text { ATP-dependent 5'-3' DNA helicase activity and role in box } \\
\text { C/D snoRNP assembly, histone exchange, rRNA processing }\end{array}$ \\
\hline-0.59 & ANIA_01971 & $\begin{array}{l}\text { Ortholog(s) have ATP-dependent 3'-5' DNA helicase activity, } \\
\text { ATP-dependent 5'-3' DNA helicase activity, sequence- } \\
\text { specific DNA binding activity }\end{array}$ \\
\hline-0.58 & ANIA_02068 & Uncharacterized \\
\hline-0.56 & ANIA_04197 & Uncharacterized \\
\hline-0.55 & ANIA_02436 & $\begin{array}{l}\text { AclB, putative ATP citrate synthase with a predicted role in } \\
\text { TCA intermediate metabolism }\end{array}$ \\
\hline-0.54 & ANIA_07011 & Uncharacterized \\
\hline-0.54 & ANIA_07208 & SET domain protein \\
\hline-0.50 & ANIA_02435 & $\begin{array}{l}\text { AclA, putative ATP citrate synthase with a predicted role in } \\
\text { TCA intermediate metabolism }\end{array}$ \\
\hline
\end{tabular}


Only $21 \%$ of the proteins that are decreased in their abundance in $\Delta c s n E$ belong to primary metabolism. This encompasses the categories electron (e) transfer, cofactors, vitamin biosynthesis; carbohydrate metabolism and amino acid metabolism. Proteins related to transcriptional control, mRNA translation or nuclear transport are decreased in their abundance in $\triangle c s n E$.
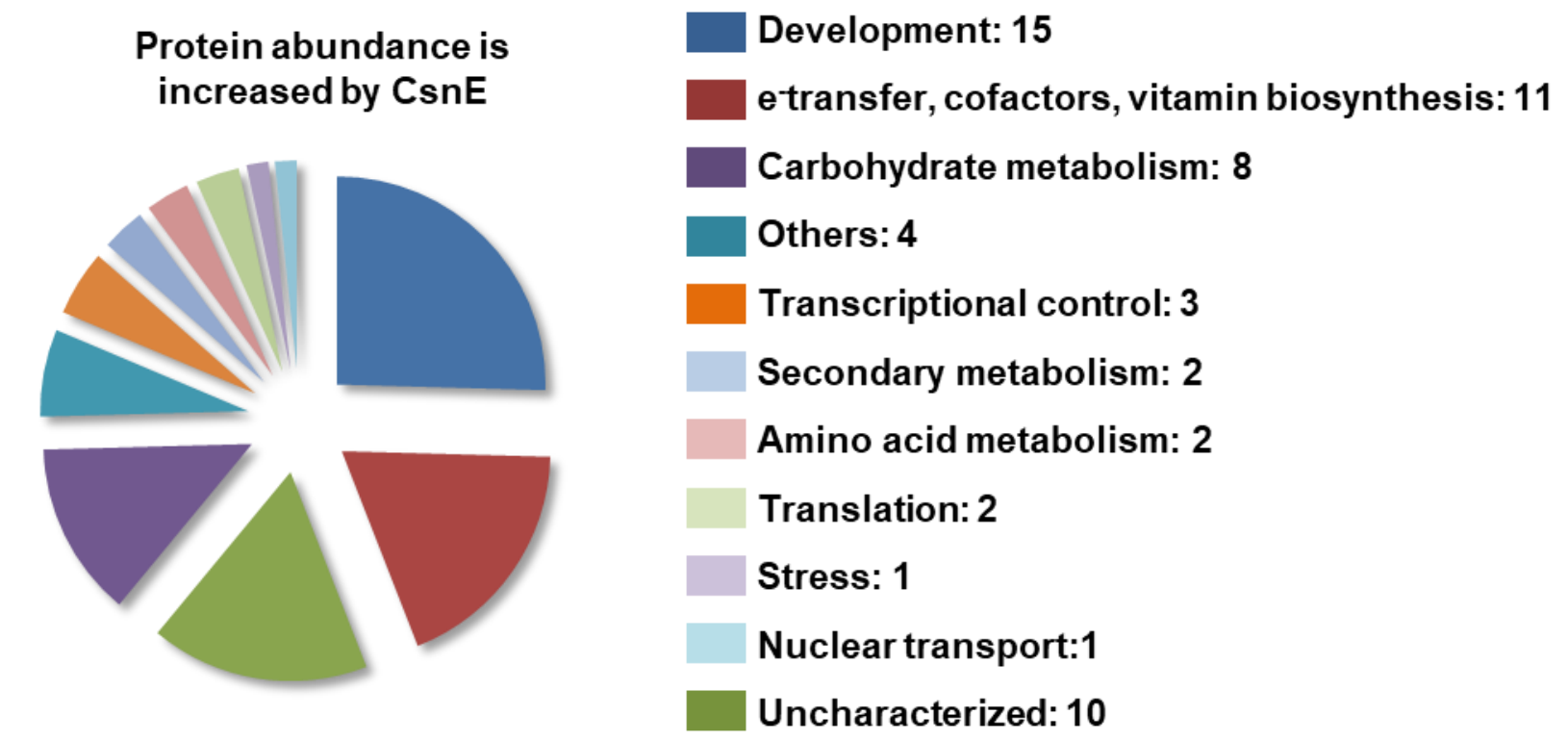

Figure 17: Classification of proteins, which abundances are increased by CsnE.

Proteins listed in Table 12 were assigned to different categories. The number behind the category indicates the number of proteins assigned to this class. One fourth of all proteins decreased in the abundance by the presence of CsnE were classified to fungal development.

In summary, relative quantification of protein abundances using SILAC is applicable with the filamentous fungus $A$. nidulans using an L-lysine auxotrophic strain. Abundances of $13.2 \%$ of the proteins identified in this study are changed in a csnE deletion mutant during vegetative growth conditions. Thereby, CsnE decreases the abundance of approximately $5 \%$ of these proteins, which were categorized to approximately two thirds to primary metabolism. Furthermore, nearly $8 \%$ of the identified proteins were increased in their abundance by CsnE. Thereby, four septins and proteins involved in hyphal growth or cytoskeleton related proteins were identified. Through the regulation of the UPS by deneddylation of CRLs, CsnE influences the abundance of proteins already during fungal vegetative growth conditions.

Vegetative growth in the presence of a functional COP9 signalosome supports an increased steady state level of specific developmental proteins and a reduced steady state level of proteins with a role in amino acid metabolism as major effect on the fungal proteome. 


\subsection{A. nidulans ubiquitin-specific protease A interacts with the COP9 signalosome}

The COP9 signalosome regulates the dynamic protein degradation process already during the vegetative, hyphal growth phase of $A$. nidulans (see Chapter 3.1). Specific and timely coordinated protein synthesis and degradation gains even more importance during the multicellular development, when $A$. nidulans reacts on external and internal stimuli or stressors to initiate the respective asexual or sexual differentiation program with the appropriate secondary metabolism (Adams et al., 1998; Axelrod et al., 1973; Braus et al., 2010; Krijgsheld et al., 2011). csn mutant strains are unresponsive to light and initiate the sexual developmental program under illumination (Busch et al., 2007). They are unable to develop mature cleistothecia. Furthermore, secondary metabolism is altered in csn mutants (Beckmann et al., 2015; Busch et al., 2007; Nahlik et al., 2010).

The COP9 signalosome physically interacts with SCF E3 ligase complexes, which do not carry a substrate, to remove the Nedd8 modification from the cullin scaffold protein (Choo et al., 2011; Mosadeghi et al., 2016). This induces conformational changes in the SCF complex that finally allow the exchange of the receptor complexes (Choo et al., 2011; Dubiel et al., 2015; Mosadeghi et al., 2016). Furthermore, the human COP9 signalosome interacts with the ubiquitin-specific protease Usp15 (Hetfeld et al., 2005). Usp15 belongs to the group of deubiquitinating enzymes, which can reverse the function of CRLs by removing ubiquitin molecules or chains from substrates (Hetfeld et al., 2005; Nijman et al., 2005). The role of these deubiquitinating enzymes in $A$. nidulans development or secondary metabolism is currently not well understood. Six different DUB families exist in human and representatives of five families could be identified in A. nidulans in silico as well (see Chapter 4.2). The largest DUB family in mammals and also in other fungi like Saccaromyces cerevisiae or Schizosaccaromyces pombe is the family of ubiquitin-specific proteases. The human Usp15, which interacts with the COP9 signalosome, belongs to this DUB family.

Basic local alignment search tool (BLAST) analyses of human Usp15 against the RefSeq database of $A$. nidulans was performed (Altschul et al., 1990). It revealed nine proteins, which have putative ubiquitin-specific protease activity according to FungiDB (Stajich et al., 2012). AN6354 was identified as best hit, followed by AN2072 and AN3711 (Table 13). Only one of these nine A. nidulans proteins, AN3587/CreB, is characterized so far (Alam and Kelly, 2017; Lockington and Kelly, 2002). Its deubiquitinating activity is important in the carbon catabolite repression process (Alam and Kelly, 2017; Lockington and Kelly, 2001). Protein blast analyses of all nine $A$. nidulans protein sequences against $S$. pombe (taxid: 4896), S. cerevisiae (taxid: 4932) or Homo sapiens (taxid: 9606) database revealed mostly enzymes with 
deubiquitination activity (Table 13). Only in silico analysis of AN4458 revealed proteins with different functions as best hit in other organisms.

Table 13: Putative orthologs of human Usp15 in A. nidulans and putative orthologous proteins in other organisms.

BLAST analyses (Altschul et al., 1990) of human Usp15 revealed nine candidates in $A$. nidulans that have putative ubiquitin-specific protease activity. A nidulans protein sequences were reblasted against S. pombe (taxid: 4896), S. cerevisiae (taxid: 4932) or H. sapiens (taxid: 9606) databases and proteins with the lowest E-value are shown.

\begin{tabular}{|c|c|c|c|c|}
\hline Systematic Name & Description & S. pombe & S. cerevisiae & H. sapiens \\
\hline AN6354 & $\begin{array}{c}\text { UspA } \\
\text { (this study) }\end{array}$ & Ubp12 & UBP12 & USP15 \\
\hline AN2072 & uncharacterized & Ubp4 & UBP5 & USP8 \\
\hline AN3711 & uncharacterized & Ubp8 & UBP8 & USP22 \\
\hline AN3587 & $\begin{array}{c}\text { CreB (Lockington } \\
\text { and Kelly, 2001) }\end{array}$ & Ubp9 & UBP13 & USP12 \\
\hline AN4458 & uncharacterized & Ubp10 & SAD1 & $\begin{array}{c}\text { U4/U6.U5 snRNP } \\
\text { associated protein 2 }\end{array}$ \\
\hline AN5186 & uncharacterized & Ubp3 & UBP3 & USP10 \\
\hline AN7422 & uncharacterized & Ubp14 & UBP14 & USP5 \\
\hline AN6913 & uncharacterized & Ubp11 & UBP1 & USP30 \\
\hline AN2507 & uncharacterized & Ubp3 & UBP10 & USP10 \\
\hline
\end{tabular}

Ubiquitin-specific proteases represent the largest DUB family in $A$. nidulans (see Chapter 4.2). USPs are cysteine proteases, which contain a catalytic triad consisting of a cysteine, a histidine and an aspartate/asparagine residue to hydrolyze the isopeptide bond between ubiquitin molecules or between an ubiquitin molecule and target proteins (Amerik and Hochstrasser, 2004). Multiple sequence alignments of the putative $A$. nidulans USPs listed in Table 13 were performed using the Clustal Omega multiple sequence alignment tool (Chenna et al., 2003). This revealed strong conservation of the cysteine $(\mathrm{C})$, histidine $(\mathrm{H})$ and aspartate $(\mathrm{D})$ residue, which build the catalytic triad in human Usp15 (Hetfeld et al., 2005). Except AN4458, which orthologs were also assigned to other functions than deubiquitination activity, all putative USPS show conservation of the three residues and also high similarities between the surrounding amino acids (Figure 18, green arrows). The total length of the fungal USPs is quite diverse ranging from 488 amino acids (AN3711) to 1,418 amino acids (AN6354). The conserved cysteine residue is rather located in the $\mathrm{N}$-terminal half of the proteins. The histidine and aspartate 
residues are located close to the C-terminus. The putative catalytic cysteine and histidine residues are separated by at least 300 amino acids from each other in most proteins, whereas the distance between the histidine and the aspartate residues are less than 100 residues.

A zinc finger motif was identified in some human USPs (Nijman et al., 2005). Human Usp15 contains a Cys4 zinc finger motif, which is essential for its deubiquitination activity (Hetfeld et al., 2005). These cysteine residues are located in between the cysteine and histidine of the catalytic triad. Multiple sequence alignments with putative $A$. nidulans USPs and human ubiquitin-specific protease 15 (Usp15) were performed to investigate if the cysteine residues are conserved in fungal DUBs (Figure 18, orange arrows).
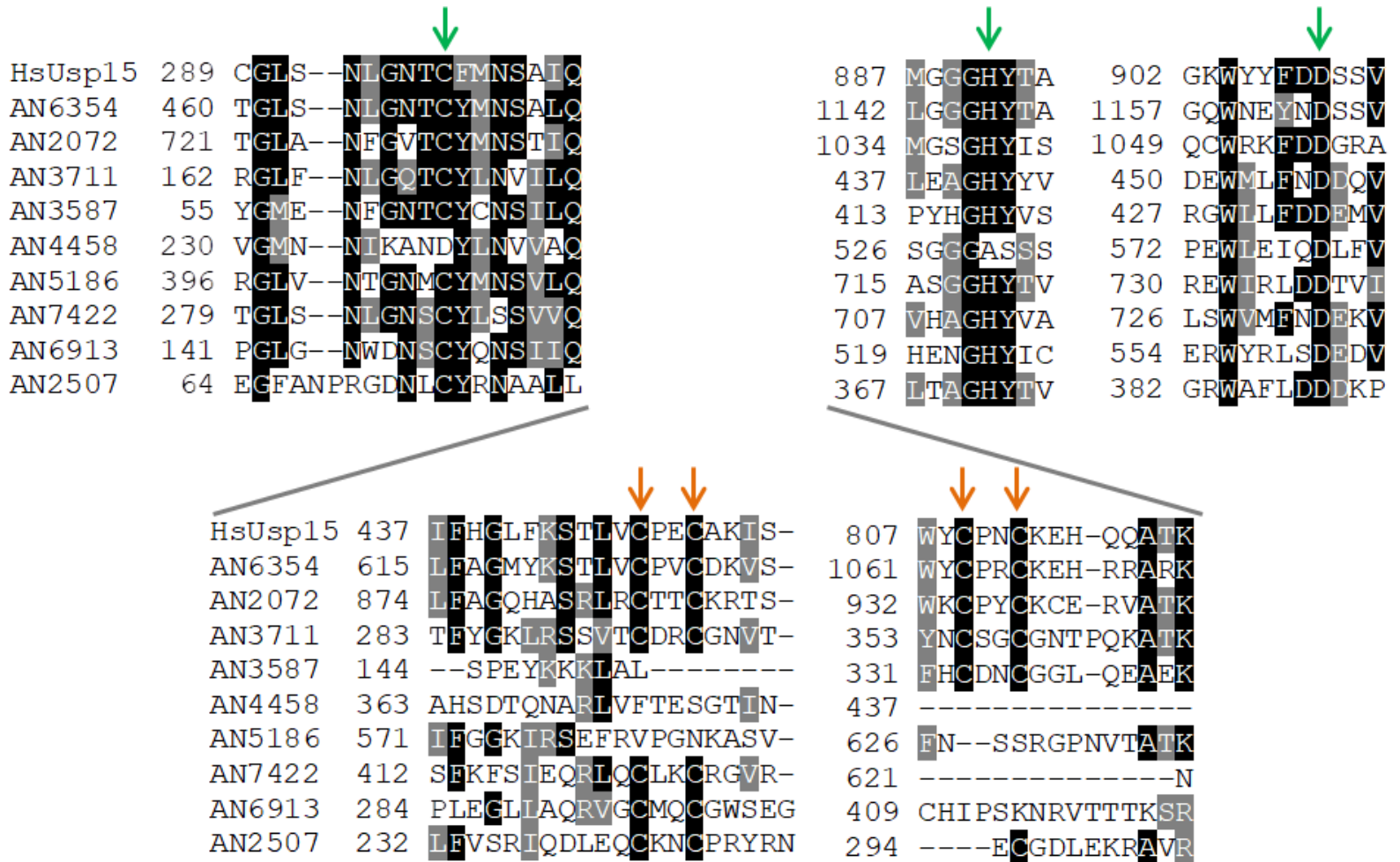

Figure 18: Multiple sequence alignments of fungal USPs.

Protein sequences from putative fungal USPs derived from FungiDB and the sequence of human Usp15 derived from Uniprot (Q9Y4E8) were used for the alignment (Stajich et al., 2012). The multiple sequence alignment was performed with Clustal Omega Software (Chenna et al., 2003) and for the coloring of the amino acids according to sequence similarities the BoxShade tool was used. The cysteine, histidine and aspartate residues, which constitute the catalytic triad are highlighted with green arrows. The cysteine residues that might form a zinc finger motif are highlighted with orange arrows. Identical residues are highlighted in a black box, similar amino acids have a grey background.

Three USPs, namely AN6354, AN2072 and AN3711 share a conserved pattern with human Usp15 of the four conserved cysteine residues (Figure 18, orange arrows). Three other proteins 
(AN3587, AN4458 and AN5186) do not contain a single conserved cysteine residue at these positions. The other proteins show partial conservation of two to three cysteine residues. They might constitute another zinc finger motif like for example $\mathrm{C}_{2} \mathrm{H}_{2}$ zinc finger. The cysteine residues constituting the zinc finger motif are situated in between the cysteine and histidine residues of the catalytic triad for all fungal USPs used in this alignment (Figure 18).

The highest amino acid sequence identities exist between human Usp15 and AN6354 with $31.47 \%$. Among the putative fungal USPs, AN6354 and AN3711 exhibit the highest sequence identity with $25.92 \%$. Most others fungal USP members have less than $20 \%$ identity to each other. The A. nidulans ortholog of human Usp15 is AN6354 and is named according to the corresponding nomenclature ubiquitin-sppecific protease $\underline{A}$ (UspA).

\subsubsection{The ubiquitin-specific protease A (UspA) encoding gene is located on chromosome I and encodes a 1,418 amino acid encompassing protein}

The fungal AN6354 open reading frame (ORF) is located on chromosome I and encompasses 4,348 nucleotides including two introns (Figure 19A). The gene encodes a protein of 1,418 amino acids with a molecular mass of $156.6 \mathrm{kDa}$.

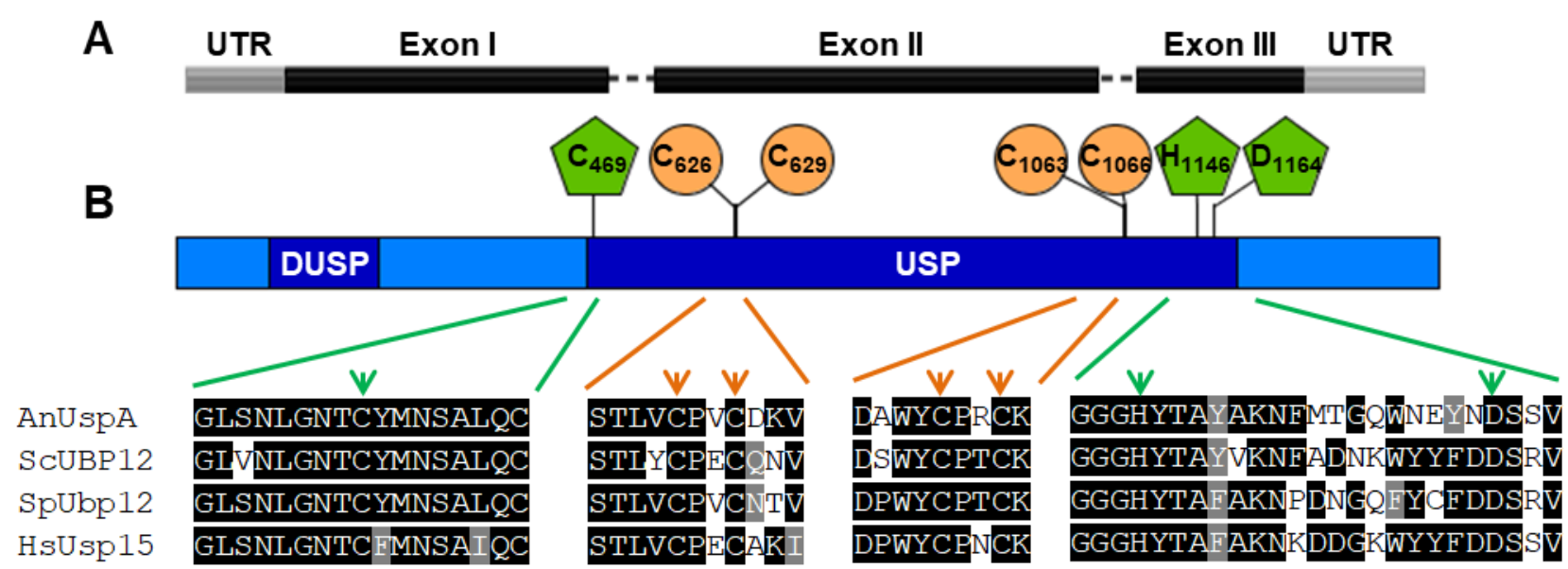

Figure 19: AN6354 encodes UspA and is a member of the fungal USP family.

A) AN6354 encodes a putative USP and is located on chromosome I. The ORF encompasses $4,348 \mathrm{bp}$ including two introns with $47 \mathrm{bp}$ or $44 \mathrm{bp}$, respectively. The 5 ' untranslated region (UTR) is composed of $445 \mathrm{bp}$, whereas the 3' UTR consists of $540 \mathrm{bp}$. B) The A. nidulans UspA consists of 1,418 amino acids. It carries the $\mathrm{N}$-terminal domain present in ubiquitin-specific proteases (DUSP). The residues of the catalytic triad $\left(C_{469}, H_{1146}\right.$ and $D_{1164}$, highlighted with green arrows) as well as the zinc-finger motif cysteine residues $\left(\mathrm{C}_{626}, \mathrm{C}_{629}, \mathrm{C}_{1063}\right.$ and $\mathrm{C}_{1066}$, highlighted with orange arrows) are located in the 730 amino acid long ubiquitin-specific protease domain (USP) and are well conserved in S. cerevisiae, S. pombe and H. sapiens. The alignment was done with the Clustal Omega software (Chenna et al, 2003) and colored with the BoxShade tool. Black boxes indicate conserved amino acids, grey boxes indicate similar amino acids and white background indicates no conservation. 
The protein contains characteristic USP domains: a domain specific for ubiquitin-specific proteases (DUSP) and an extended C-terminal ubiquitin-specific protease (USP) domain (Figure 19). The catalytic triad in UspA is constituted by the cysteine residue (C469) in the $\mathrm{N}$-terminal half of the USP domain as well as a histidine $(\mathrm{H} 1146)$ and an aspartate residue (D1164) near the C-terminus of the USP domain. These residues and surrounding amino acids are well conserved in orthologous proteins in $S$. pombe, $S$. cerevisiae and $H$. sapiens (Figure 19). The cysteine residues constituting the zinc finger motif in Usp15 are well conserved in orthologous proteins of $A$. nidulans (C626, C629, C1063, C1066), S. pombe and S. cerevisiae as well (Figure 19).

The whole amino acid sequence of $A$. nidulans UspA includes $35.33 \%$ identical amino acid residues to the $S$. pombe ortholog Ubp12 according to sequence alignments with the Clustal Omega software (Chenna et al., 2003). Sequence identities to the S. cerevisiae ortholog UBP12 and $H$. sapiens Usp15 isoform 2 are approximately $30 \%$ according to the Clustal Omega alignment tool (Chenna et al., 2003).

\subsubsection{A. nidulans UspA interacts with six subunits of the COP9 signalosome in a yeast- two-hybrid assay}

The COP9 signalosome is an eight subunit protein complex conserved from fungi to human (Braus et al., 2010). It deneddylates CRLs and plays therefore a role in the ubiquitin-proteasome pathway (Cope and Deshaies, 2006; Lyapina et al., 2001). The S. pombe ortholog Ubp12 was isolated together with the COP9 signalosome from cell lysates (Zhou et al., 2003). Furthermore, Hetfeld and colleagues co-purified the human ortholog Usp15 together with the COP9 signalosome from human erythrocytes (Hetfeld et al., 2005). A yeast-two-hybrid (Y2H) assay was performed to study the interaction of UspA with the COP9 signalosome subunits of A. nidulans. uspA cDNA served as bait $\left(u s p A^{b}\right)$ and was fused to the lexA DNA-binding domain under the control of the strong alcohol dehydrogenase promotor. Single csn subunits served as prey and were $\mathrm{N}$-terminally fused to the activation domain under the control of the inducible GAL1 promoter (Figure 20A). The GAL1 promoter is strongly repressed in presence of glucose and can only activate gene transcription if an alternative carbon source such as galactose is in the medium (Flick and Johnston, 1990). Bait and prey plasmids were co-transformed into the yeast strain EGY48 (Golemis et al., 2001). This strain contains two reporter genes that are responsive to the lexA operator system: the LEU2 and the lacZ reporter genes. The transcription of the LEU2 gene enables growth on medium without leucine, whereas transcription of lacZ 
leads to blue colored colonies on medium supplemented with 5-bromo-4-chloro-3-indoxyl- $\beta$-Dgalactopyranosid (X-Gal) (Golemis et al., 2001).

In the following experiment only the transcription of the LEU2 reporter gene was monitored on medium lacking leucine (Figure $20 \mathrm{~B})$. The empty bait vector $\left(\mathrm{EV}^{\mathrm{b}}\right)$ was expressed together with the empty prey vector $\left(E V^{\mathbb{P}}\right)$ as negative control. Additionally, the Usp $A^{b}$ was expressed together with the $E V^{p}$ to exclude auto-activation of the $L E U 2$ reporter gene through the mere bait. UspA ${ }^{b}$ was tested together with each of the single CSN subunits as prey $\left(\mathrm{CsnA}-\mathrm{H}^{\mathrm{p}}\right)$.

A
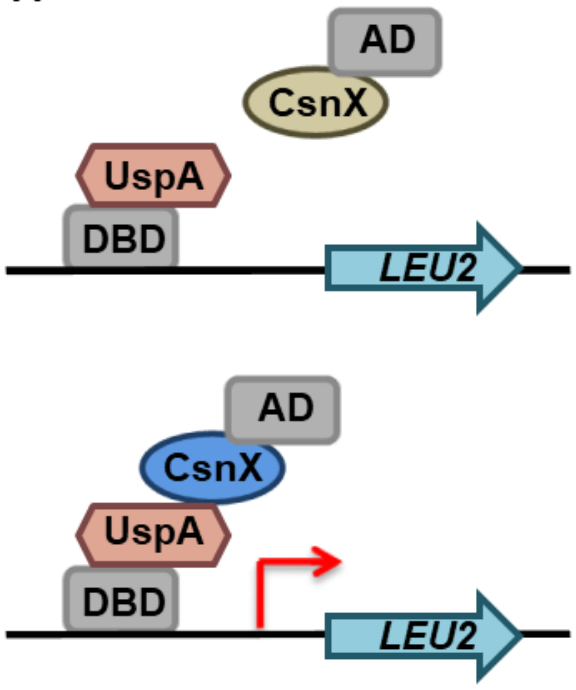

B

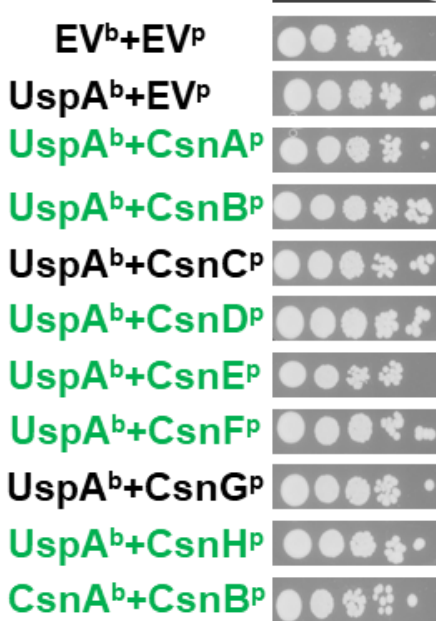

Gluc +Leu Gluc -Leu Gal/Raf -Leu

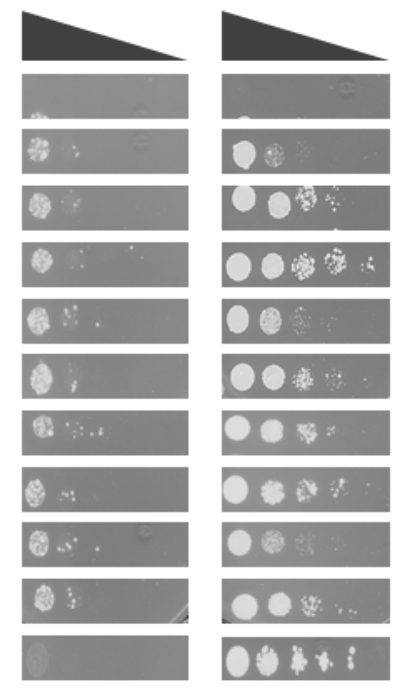

Figure 20: A. nidulans UspA interacts with COP9 signalosome subunits in yeast-twohybrid assay.

A) A schematic representation of the induction of the LEU2 reporter gene is shown. UspA was fused to the lexA DNA binding domain (DBD), the single CSN subunits were fused to the activation domain (AD) of the GAL4 transcription factor. In case of interaction of UspA and the respective CSN subunit, $D B D$ and $A D$ come into close proximity and initiate the transcription of the LEU2 reporter gene. B) Yeast strain EGY48 was co-transformed with the bait vector containing uspA $\left(u s p A^{b}\right)$ fused to the lexA DBD and a prey vector containing one of the CSN subunits genes fused to the $A D\left(\operatorname{csn} X^{p}\right)$, respectively. $C s n A^{b}$ with $C s n B^{p}$, which are known to interact in $\mathrm{Y} 2 \mathrm{H}$ experiments, served as positive control (Busch et al., 2007). Medium with glucose and leucine served as positive control where all strains including the negative controls with the empty vectors $\left(E^{2} / E V^{p}, U s p A^{b} / E^{p}\right)$ grow (left panel). The level of auto-activation of the LEU2 reporter by the bait protein is shown on medium with glucose lacking leucine (middle panel). Only strains with interacting bait and prey proteins are able to grow on the interaction medium containing galactose and raffinose as carbon sources. Combinations of interacting proteins are highlighted in green. The gradient represents different dilutions of yeast cells that were used for the spotting. 
All yeast strains were able to grow on medium containing glucose as carbon source and leucine (Gluc +Leu), which served as positive control (Figure 20B, left panel). Lack of leucine (Gluc Leu) served as negative control medium and prevented growth of the $\mathrm{EV}^{\mathrm{b}} / \mathrm{EV}^{\mathrm{p}}$ containing yeast strain (Figure 20B, middle panel). All other strains show some background growth. This indicates a certain level of auto-activation of the LEU2 reporter through the bait only. Medium without leucine and galactose/raffinose as carbon source (Gal/Raf -Leu) should enable growth of strains with interacting proteins only (Figure 20B, right panel). Cells expressing Usp $\mathrm{A}^{\mathrm{b}}$ together with $\mathrm{CsnB}^{\mathrm{p}}$ showed a strong growth similar to the positive control $\mathrm{CsnA}^{\mathrm{b}} / \mathrm{CsnB}^{\mathrm{p}}$ indicating an interaction of these proteins. Furthermore, strains expressing $U s p A^{b} / C s n A^{p}, U s p A^{b} / C_{s n D}{ }^{p}$, $\mathrm{UspA}^{\mathrm{b}} / \mathrm{CsnE} \mathrm{E}^{\mathrm{p}}, \mathrm{UspA}^{\mathrm{b}} / \mathrm{CsnF}^{\mathrm{p}}$ and $\mathrm{UspA}^{\mathrm{b}} / \mathrm{CsnH}^{\mathrm{p}}$ grew better on interaction medium (Gal/Raf -Leu) than on the control plates indicating an interaction of these proteins. The $\mathrm{Y} 2 \mathrm{H}$ assay did not reveal a direct physical interaction between $U s p A^{b}$ and $C_{s n C}{ }^{p}$ or $C_{s n G}{ }^{p}$ (Figure 20B). In summary, direct physical interactions between UspA and six subunits of the COP9 signalosome, namely CsnA, B, D, E, F and $\mathrm{H}$ were observed in the $\mathrm{Y} 2 \mathrm{H}$ assay.

\subsubsection{CsnE causes repressed transcript levels of the UspA encoding gene}

Single CSN subunits interact with UspA in a $\mathrm{Y} 2 \mathrm{H}$ assay. The COP9 signalosome regulates with its deneddylation activity CRLs, which catalyze the ubiquitination of substrates (Beckmann et al., 2015; Hua and Vierstra, 2011). The putative deubiquitinase UspA might counteract this process or could rescue CRL components from ubiquitination and subsequent degradation. The COP9 signalosome and UspA might at least temporary regulate the CRLs or their substrates together. A csnE deletion strain lacks the catalytically active subunit of the COP9 signalosome and this leads to accumulation of constantly neddylated or hyperneddylated cullins, which might ubiquitinate proteins unspecifically or autoubiquitinate CRL components (Cope and Deshaies, 2006; Hua and Vierstra, 2011). Gene expression levels of deubiquitinating enzymes were analyzed with quantitative real-time PCR (qRT-PCR) in $\Delta c s n E$ strains compared to wild type during different developmental stages to investigate how the fungal cell reacts in this situation.

RNA was isolated from wild type and $\triangle c s n E$ strains grown vegetatively, asexually or sexually and cDNA was synthesized. Four genes encoding ubiquitin-specific proteases found in initial in silico analyses were chosen for this experiment (Table 13). AN6354/uspA was used due to the direct interaction of its gene product with COP9 signalosome subunits. Furthermore, AN3711 was chosen as its gene product shows highest similarity to AN6354/UspA among fungal USPs. $A N 3587 /$ creB is the only already described USP in A. nidulans (Alam and Kelly, 2017; 
Lockington and Kelly, 2002). AN2072 was among the best hits of the BLAST analysis of human Usp15 against $A$. nidulans database and the encoding gene AN2072 was therefore included in this analysis (Table 13). Transcript levels of genes encoding putative deubiquitinating enzymes are not changed between wild type and $\triangle c s n E$ strains during vegetative growth (Figure 21).

Growth under asexual or sexual development inducing conditions led to an upregulation of putative USP encoding genes in $\triangle c s n E$. AN6354/uspA shows thereby the highest upregulation of all tested genes. More than four times higher expression of uspA in $\triangle c s n E$ compared to wild type was observed during asexual development inducing conditions and around three times upregulation during sexual development inducing conditions.
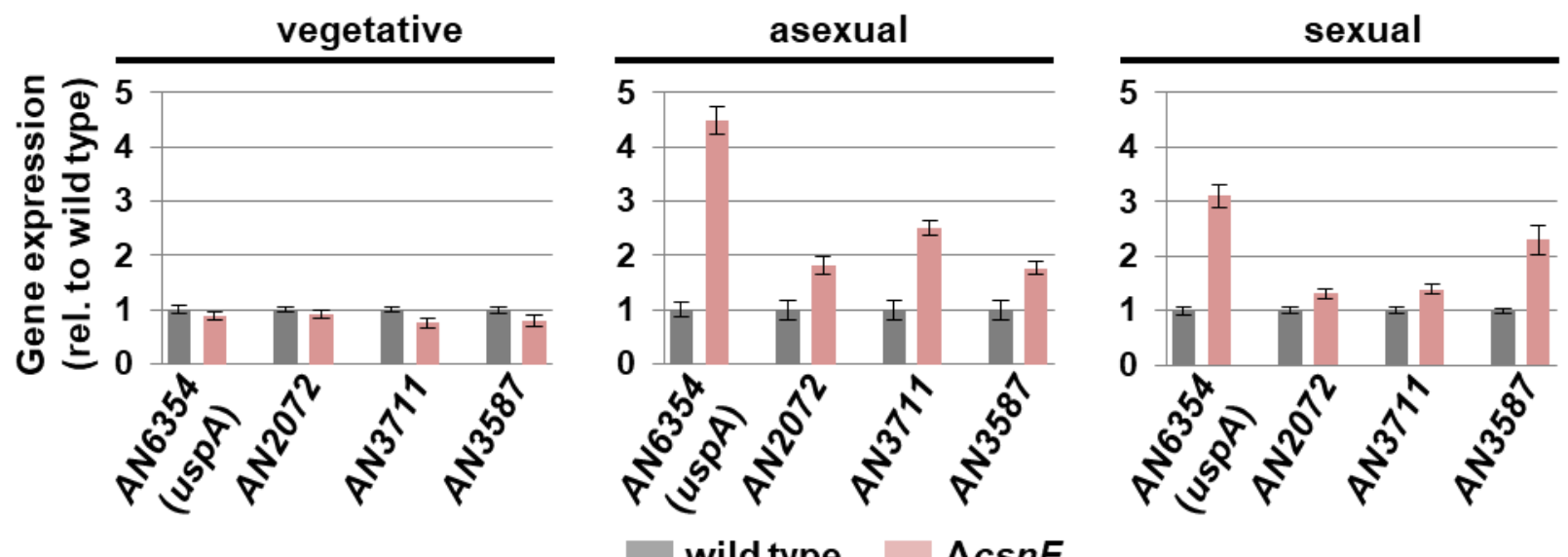

Figure 21: CsnE causes repressed expression of genes encoding for putative USPs during multicellular development of $\boldsymbol{A}$. nidulans.

qRT-PCR indicates that the transcript levels of putative USPs are derepressed in the absence of CsnE during asexual and sexual development. RNA derived from mycelia grown for $20 \mathrm{~h}$ in submerged culture at $37^{\circ} \mathrm{C}$, or from asexually or sexually grown mycelia was used. As reference genes served $h 2 A$ and $15 S r R N A$. Wild type expression was set to 1 . Error bars represent standard error of the mean (SEM) of at least two biological replicates.

AN3711 encodes the most similar protein to UspA and shows the second highest upregulation in the csnE defective strain during asexual development. In general, all tested transcripts for putative USPs were upregulated in csnE deletion strain during multicellular development. Yet, only a minor upregulation was observed for AN2072 and AN3711 during sexual development, respectively. This indicates that the presence of constantly neddylated CRLs due to a defective COP9 signalosome in $\triangle c s n E$ strains leads to increased expression of putative USPs, especially of $u s p A$, during $A$. nidulans multicellular development. 


\subsection{4 csnE transcript level and CsnE protein stability are independent of UspA}

A well-studied function of ubiquitin chains is denoting substrates for degradation by the $26 \mathrm{~S}$ proteasome ( $\mathrm{Li}$ and Ye, 2008). Deubiquitinating enzymes reverse the ubiquitination process and can protect substrates from degradation (Kim et al., 2003; Nandi et al., 2006; Nijman et al., 2005). Substrates of UspA are supposed to be less stable in $\Delta u s p A$ strains as the ubiquitin chains cannot be removed anymore. UspA seems to interact with CSN subunits in $A$. nidulans. Its human ortholog Usp15 protects substrates for degradation by the 26S proteasome while interacting with the COP9 signalosome (Schweitzer et al., 2007). In this study it was analyzed if UspA has any direct influence on the catalytically active subunit CsnE of the COP9 signalosome. Therefore, transcript levels of $c s n E$ and corresponding protein stability of CsnE were analyzed in an $u s p A$ deletion strain compared to wild type during fungal development.

Therefore, RNA was isolated and cDNA synthesized from wild type, $\Delta u s p A^{\text {pyroA }}$ and comp $p^{\text {pyroA }}$ strains that were grown vegetatively, asexually or sexually. qRT-PCR experiments showed that the transcript levels of $c s n E$ were not changed in the absence of the deubiquitinase during all developmental stages (Figure 22).

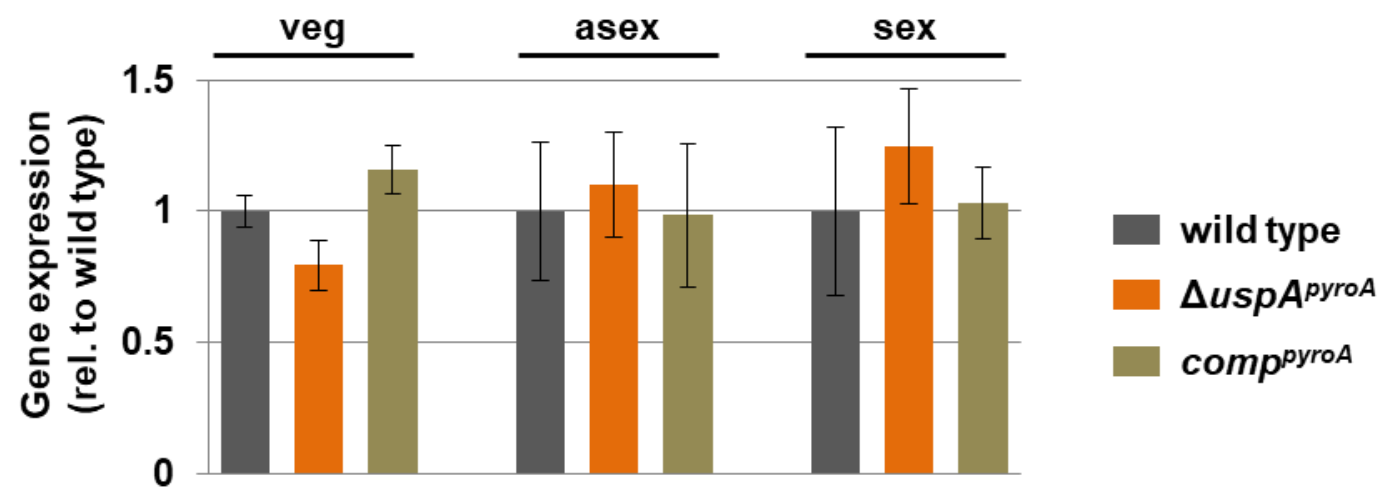

Figure 22: csnE transcript levels are independent of UspA.

Strains were vegetatively grown for $20 \mathrm{~h}$ in liquid culture (veg) or mycelia were subsequently transferred onto agar plates to induce asexual (asex) or sexual (sex) development. Asexually grown samples were harvested after $24 \mathrm{~h}$, sexually grown samples were harvested after $48 \mathrm{~h}$ of development. RNA was isolated and cDNA was synthesized. CDNA was used for qRT-PCR experiments. Wild type expression levels were set to 1 , as reference genes served $h 2 A$ and $15 S$ rRNA. Vegetative expression levels resulted from one biological and three technical replicates, whereas asexual and sexual expression levels derived from two independent biological replicates with three technical replicates, respectively. Error bars represent the standard error of the mean.

DUBs rather influence the abundance of proteins due to their ability to remove the posttranslational modification ubiquitin from proteins and can protect them from proteasomal 
degradation (Kim et al., 2003; Nijman et al., 2005). Therefore, protein levels of CsnE were analyzed throughout fungal development. A control strain harboring a wild type uspA ORF and the $\triangle u s p A^{p y r o A}$ strain were grown vegetatively for $20 \mathrm{~h}$ in submerged culture at $37^{\circ} \mathrm{C}$ and subsequently mycelia were shifted on solid agar plates to investigate protein levels of CsnE during initiation of multicellular fungal development. The agar plates were incubated in asexual (light and oxygen) or sexual development (darkness and carbon dioxide pressure) inducing conditions. Protein crude extracts were prepared from mycelium from all different time points and western hybridization experiments with aCsnE antibody were performed (Figure 23).
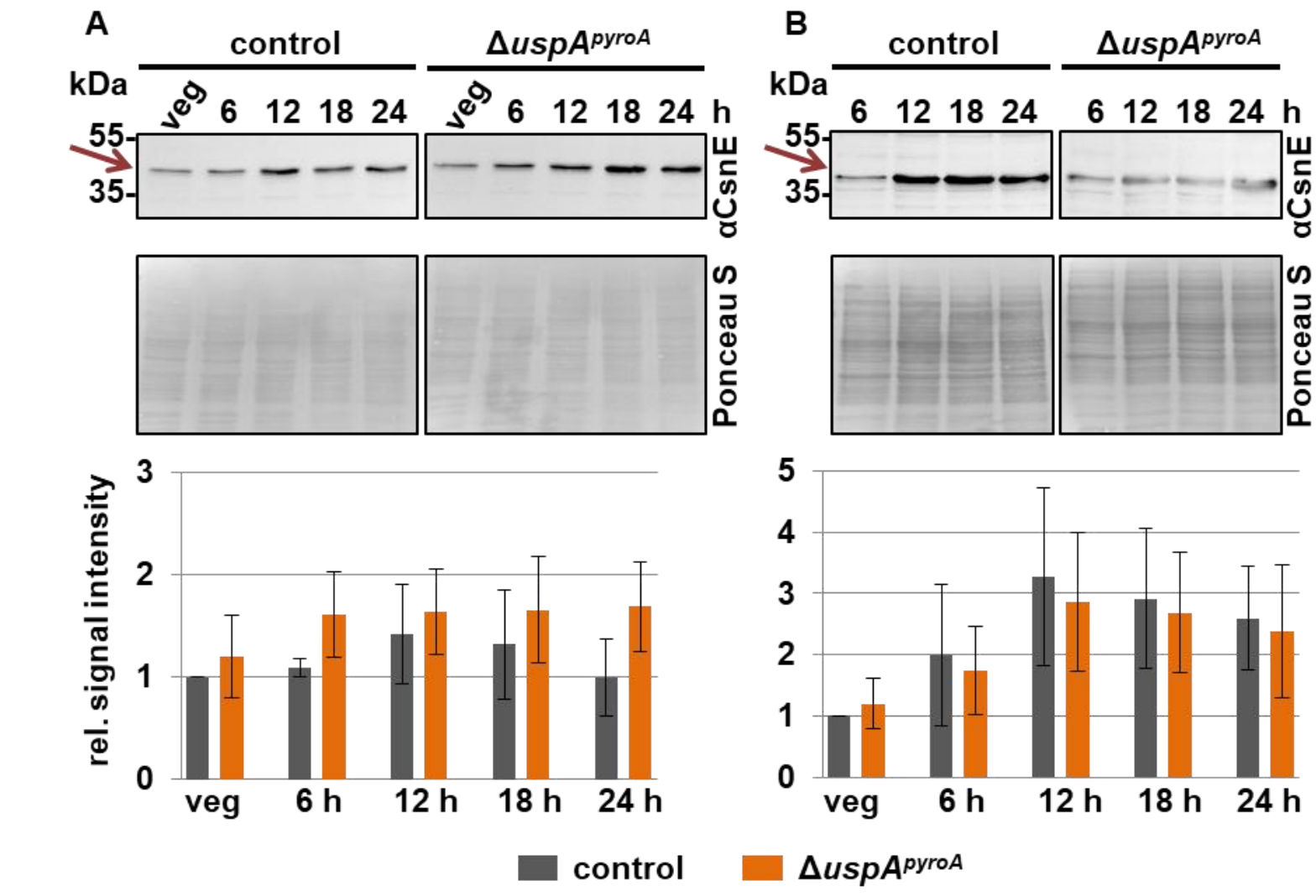

Figure 23: CsnE protein levels are independent of the deubiquitinase UspA.

Western hybridization experiments of total fungal protein crude extracts of an uspA deletion strain or a strain, which expresses wild type uspA levels. A) Strains were incubated for up to $24 \mathrm{~h}$ in asexual development inducing conditions. B) Strains were incubated for up to $24 \mathrm{~h}$ in sexual development inducing conditions. Fungal CsnE has a molecular weight of approximately $38 \mathrm{kDa}$ and the respective signal is highlighted with a red arrow. CsnE signals were normalized against the Ponceau $S$ loading control. Error bars represent the standard error of the mean (SEM) of three independent biological replicates. 
Western hybridization experiments with a aCsnE-specific antibody showed that the protein levels of CsnE are similar during vegetative growth and asexual development in $\Delta u s p A^{\text {pyro } A}$ strain and the control. CsnE is also stable during sexual development inducing conditions. Protein levels do not differ in the control compared to uspA deletion strain. Slightly increased CsnE protein levels after $12 \mathrm{~h}$ of sexual development were observed in both strains.

Taken together, CsnE influences the transcript levels of uspA and other genes encoding for putative ubiquitin-specific proteases in $A$. nidulans during multicellular fungal development. Conversely, the transcript level and the protein abundance of the catalytically active subunit CsnE of the COP9 signalosome are not influenced by UspA.

\subsection{UspA is localized in proximity to and inside nuclei}

The localization of proteins inside the cell is often linked to their function. The subcellular distribution of the catalytic active subunit CsnE of the COP9 signalosome is highly dynamic with a population in the nucleus and another one in the cytoplasm (Tomoda et al., 2002). Thereby, CsnE is incorporated in the full COP9 signalosome complex inside the nuclei, whereas smaller subcomplexes are present in the cytoplasm (Tomoda et al., 2002). The COP9 signalosome subunit CsnD is accumulating in nuclei in A. nidulans (Busch et al., 2003). In the present study, the localization of the UspA deubiquitinase was analyzed in the fungal cell during hyphal growth conditions by fluorescence microscopy. Thereby, the interaction of UspA with COP9 signalosome subunits was examined in vivo and the effect of the catalytic activity of UspA on the subcellular localization was compared.

\subsubsection{UspA interacts with CsnB and CsnF in vivo in A. nidulans}

Bimolecular fluorescence complementation (BiFC) experiments were performed to examine interactions between the ubiquitin-specific protease UspA and subunits of the COP9 signalosome in vivo. CsnB and CsnF were chosen for BiFC experiments as both of them showed strong interacting signals in the $\mathrm{Y} 2 \mathrm{H}$ assay (Figure 20). Therefore, UspA and either CsnB or CsnF were tagged with $\mathrm{N}$ - and C-terminal halves of the yellow fluorescent protein (YFP), respectively. The separated parts of the YFP protein are not fluorescent (Figure 24). If both proteins are interacting, the two halves of the YFP protein come into close proximity and emit a fluorescent signal upon excitation. UspA was fused $\mathrm{N}$-terminally to the C-terminal part of 
YFP (cYFP-UspA), whereas CsnB or CsnF respectively were fused to the N-terminal part of YFP (nYFP-CsnB or nYFP-CsnF).

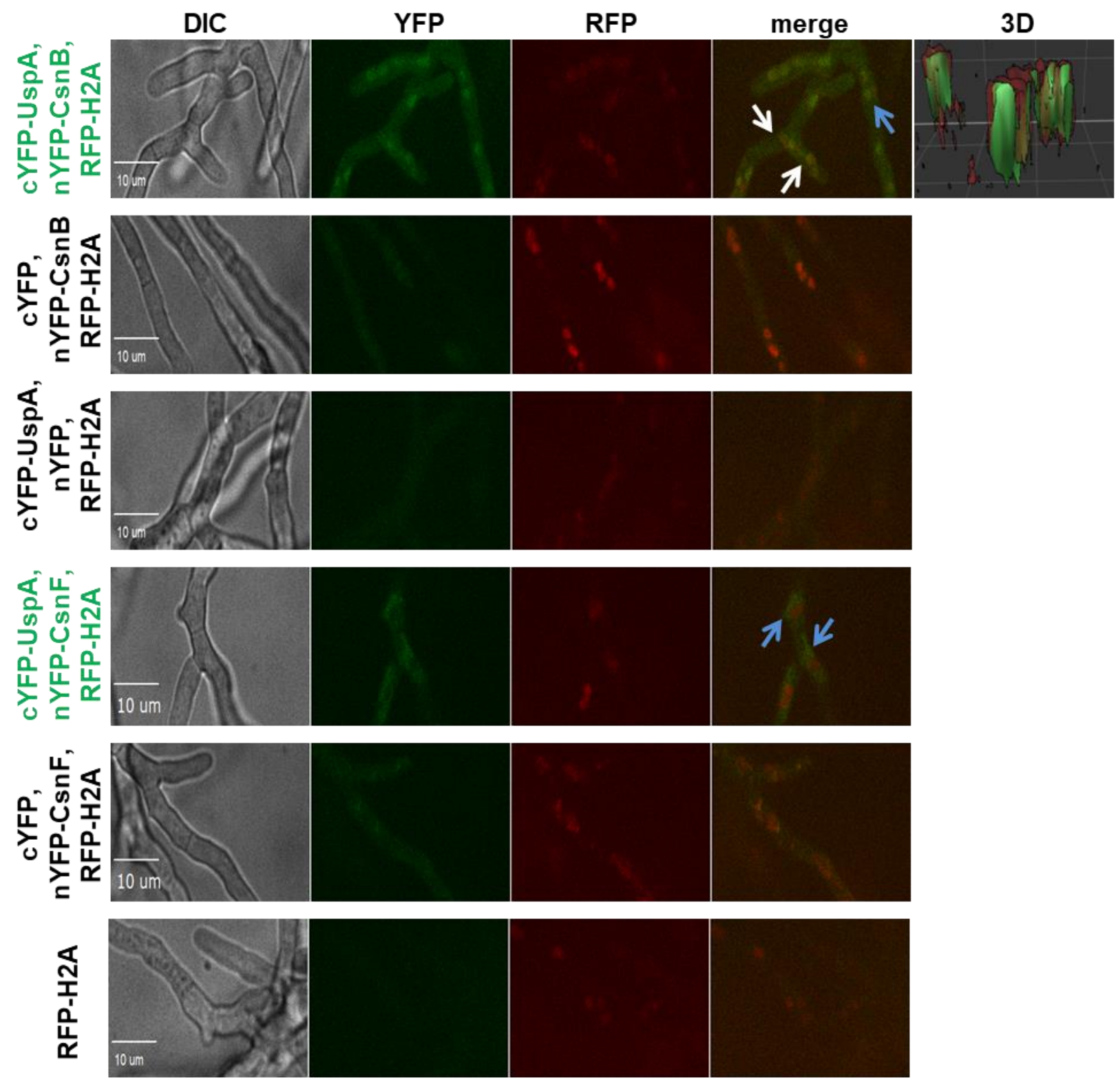

Figure 24: UspA interacts with CsnB and CsnF in bimolecular fluorescence complementation (BiFC) experiments.

BiFC experiments with strains expressing UspA fused to C-terminal half of YFP (cYFP-UspA) and CsnB or CsnF fused to the N-terminal half of YFP (nYFP-CsnB or nYFP-CsnF) were performed (respective panels are highlighted in green, control strains are labeled in black). 2,000 spores were inoculated in MM on cover slides and incubated for $20 \mathrm{~h}$ at $37^{\circ} \mathrm{C}$ in light to induce vegetative growth. YFP fluorescent signals are indicated by white arrows if they co-localize with nuclei and with blue arrows if the YFP signal was observed close to, but not inside nuclei. Nuclei show RFP fluorescent signals due to the expression of the RFP-H2A fusion protein. The parental strain used for BiFC experiments shows only RFP fluorescent nuclei (RFP-H2A). 
Either cYFP-UspA or the respective CSN subunit (nYFP-CsnB, nYFP-CsnF) fused to split YFP were expressed together with the corresponding free half of YFP (nYFP or cYFP) as control. These strains should not show any fluorescent signal as the cYFP and nYFP should not come into close proximity. The parental strain used for BiFC experiment expressed histone $\mathrm{H} 2 \mathrm{~A}$ fused to the red fluorescent protein (H2A-RFP), which leads to red fluorescing nuclei when stimulated by an appropriate light source. This enables the investigation of the intracellular localization of the protein interaction. The strain expressing cYFP-UspA and nYFP-CsnB showed fluorescent signal near and inside nuclei (Figure 24, upper panel). A three dimensional (3D) picture of nuclei confirmed that the interaction of CsnB and UspA takes place inside the nuclei. A fluorescent signal was observed also close to nuclei (Figure 24, blue arrow, upper panel). Control strains expressing either cYFP-UspA and nYFP or cYFP and nYFP-CsnB did not show any YFP fluorescence. Only red stained nuclei due to the RFP-tagged H2A were observed. Interaction of UspA and CsnF was exclusively observed around nuclei (Figure 24, blue arrow). YFP fluorescent signals were only observed in strains expressing cYFP-UspA and nYFP-CsnB or nYFP-CsnF, not in strains expressing only one protein and the empty half of the YFP (highlighted with black labeling). This confirms the interaction of UspA with CSN subunits observed in $\mathrm{Y} 2 \mathrm{H}$ experiments in $A$. nidulans in vivo.

\subsubsection{Active and inactive UspA is localized close to and within nuclei}

Functional UspA-GFP fusion protein as well as a mutant UspA-GFP fusion protein was expressed under the control of its native promotor to examine the subcellular localization of the deubiquitinase. In the gene for the mutant UspA-GFP protein the codons for two cysteine residues, which are required for its catalytic activity, were mutated (see Chapter 3.4). One cysteine residue belonging to the catalytic triad was exchanged by alanine (C469A) and one cysteine residue of the zinc finger motif was exchanged by alanine (C1066A). In the following, this construct is referred to as UspA ${ }^{A A}$-GFP. A construct containing RFP-tagged histone $\mathrm{H} 2 \mathrm{~A}$ was transformed in the UspA-GFP strains to visualize the nuclei. The GFP signal for UspA-GFP is accumulating in small spots in close proximity to nuclei. Small subpopulations are inside the nucleus and distributed through the cytoplasm as well. A 3D picture of microscopic GFP and RFP signals revealed partial overlaps of UspA with nuclei (Figure 25, middle panel). The localization of the inactive UspA-GFP mutant protein shows similar subpopulations. It accumulates close to nuclei, even though to a lesser extent than the functional fusion protein and a cytoplasmic and nuclear subpopulation also exist (Figure 25, lower panel). 
In summary, UspA is located in proximity to nuclei, whereby smaller subpopulations appear inside nuclei or are distributed through the cytoplasm. This might reflect a dynamic process, which is slightly shifted towards the cytoplasmic subpopulation when UspA is dysfunctional.

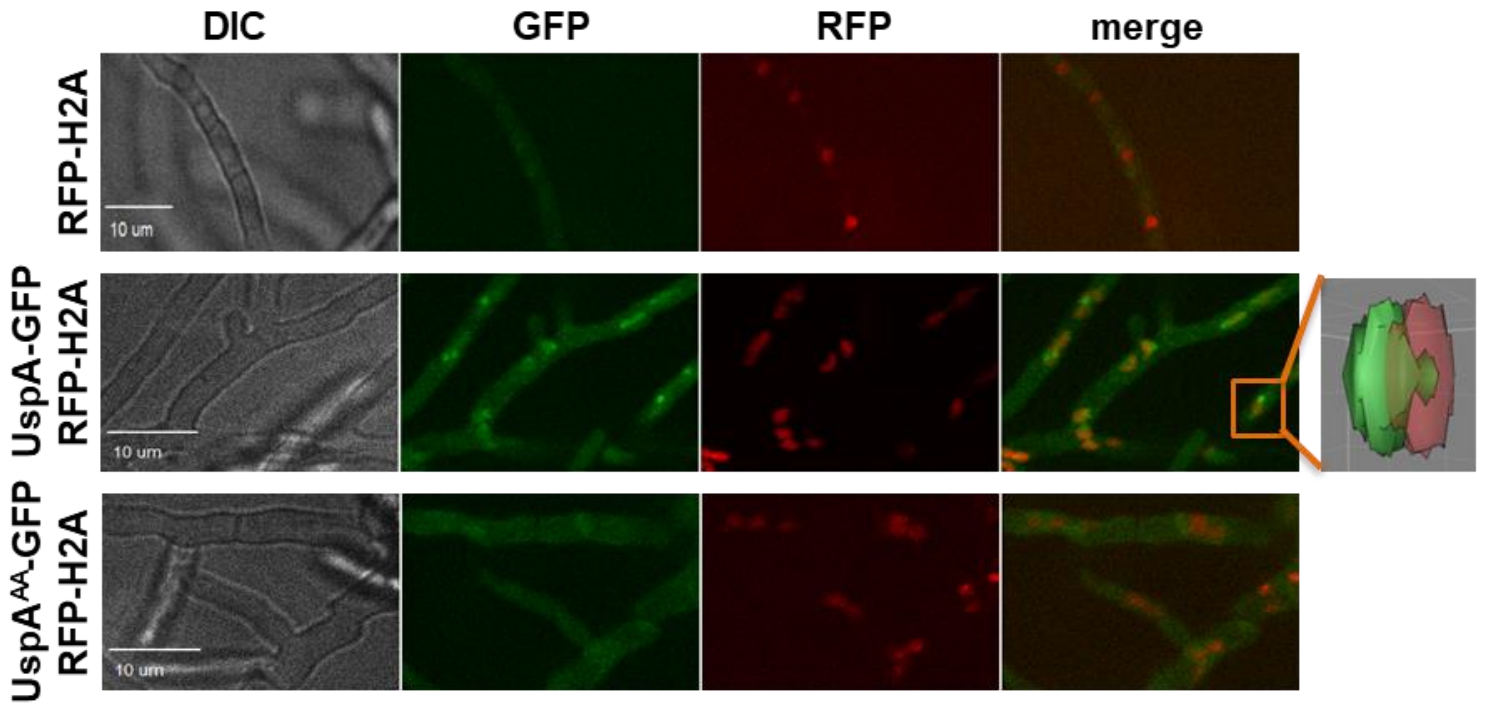

Figure 25: Functional and non-functional UspA-GFP proteins accumulate close to nuclei. 2,000 spores were inoculated for fluorescence microscopy and grown in liquid medium on cover slides for $20 \mathrm{~h}$ at $37^{\circ} \mathrm{C}$ in light. A plasmid containing RFP tagged $\mathrm{H} 2 \mathrm{~A}$ was transformed into UspA-GFP or UspA ${ }^{\mathrm{AA}}$-GFP expressing strains to visualize nuclei. GFP signal is shown in green and accumulates close to nuclei. Size bars represent $10 \mu \mathrm{m}$.

BiFC experiments confirmed that UspA interacts with the COP9 signalosome subunits CsnB and CsnF (Figure 24). An N-terminal fusion protein RFP-CsnB was constructed and transformed into strains expressing the different UspA-GFP versions. Localization of UspA and CsnB was investigated using fluorescence microscopy. A. nidulans strains expressing the active or inactive UspA-GFP and RFP-CsnB were grown in liquid medium on cover slides for $20 \mathrm{~h}$ at $37^{\circ} \mathrm{C}$ under illumination. UspA-GFP shows cytoplasmic localization with distinct accumulation points near nuclei as described before (Figure 25). The relatively weak RFP-CsnB signals were observed close to and partially overlapping with UspA-GFP signals (Figure 26A, middle panel). Inactive UspA-GFP showed a more diffuse GFP fluorescent signal throughout the cytoplasm, but some co-localization of UspA ${ }^{A A}$-GFP and RFP-CsnB was observed (Figure 26A, lower panel).

Nuclei were visualized through staining with the blue fluorescent dye Hoechst, which is able to bind DNA (Figure 26B). UspA-GFP co-localized with RFP-CsnB and Hoechst stained nuclei. This indicates that apart from the subpopulation of UspA-GFP close to nuclei, an additional subpopulation inside nuclei is present in the fungal cell, which interacts with $\mathrm{CsnB}$. In the strain expressing the inactive UspA-GFP protein, RFP-CsnB and Hoechst stained nuclei showed 
co-localization, whereas the GFP signal is more diffuse. Some accumulation of the GFP fluorescence is co-localized to the sites of RFP/Hoechst signals (indicated with white arrows).

Taken together, a subpopulation of active UspA-GFP as well as of inactive UspA ${ }^{A A}$-GFP co-localized with RFP-CsnB. This observation is in accordance with the findings that UspA interacted with CsnB in $\mathrm{Y} 2 \mathrm{H}$ and BiFC experiments. Therefore, the interaction of UspA with the COP9 signalosome and the catalytic activity of UspA are independent of each other (see Chapter 3.4).
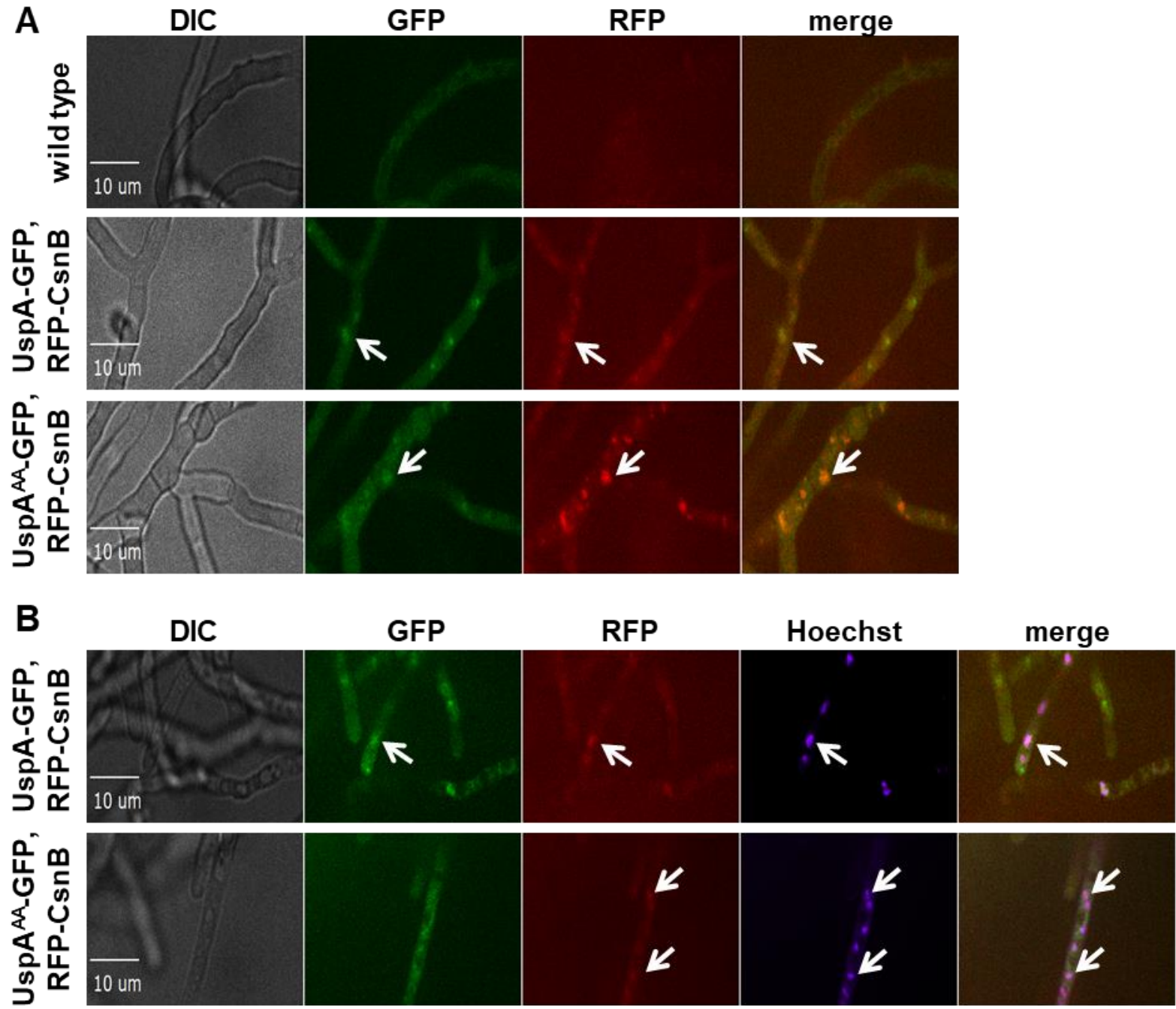

Figure 26: Subpopulations of UspA-GFP and UspA AA-GFP co-localize with RFP-CsnB.

A) 2,000 spores were inoculated in minimal liquid medium on cover slides and grown for $20 \mathrm{~h}$ vegetatively at $37^{\circ} \mathrm{C}$. Overlapping GFP and RFP signals are indicated with white arrows. B) Minimal medium was removed from the cover slides and exchanged with minimal medium containing Hoechst $(10 \mu \mathrm{g} / \mathrm{ml})$ to visualize nuclei. Samples were incubated for $15 \mathrm{~min}$ at $37^{\circ} \mathrm{C}$ before using them for fluorescence microscopy. White arrows indicate co-localization. Size bars represent $10 \mu \mathrm{m}$. 


\subsection{UspA activity requires $\mathrm{C} 469$ and $\mathrm{C} 1066$ to reduce the cellular pool of ubiquitinated proteins in the fungal cell}

The upregulation of the uspA transcript in the absence of a functional CSN complex as well as the association to certain CSN subunits to UspA in $\mathrm{Y} 2 \mathrm{H}$ and BiFC experiments indicate a role of UspA in the ubiquitin-proteasome system. Deletion strains of uspA were constructed to elucidate the function of the DUB. The $A$. nidulans uspA ORF was replaced by the pyroA marker from Aspergillus fumigatus resulting in the $\triangle u s p A^{p y r o A}$ deletion strain. This strain was complemented by ectopical integration of the $u s p A$ ORF resulting in the comp pyroA strain. Furthermore, an uspA deletion strain was constructed using a recyclable marker system in which the marker cassette can be excised off the genome and only a small six site is left as a scar. This strain was called $\Delta u s p A^{\text {Six }}$. The resulting strain was complemented by integration of the $u s p A:: g f p$ fusion construct in the $u s p A$ gene locus through homologous recombination ( $u s p A:: g f p$ ). Additionally, an UspA-GFP mutant protein was constructed, which carries amino acid exchanges: a cysteine residue belonging to the catalytic triad was mutated to alanine $(\mathrm{C} 469 \mathrm{~A})$ and one cysteine residue of the zinc finger motif was mutated to alanine (C1066A). In the following this strain is referred to as $u s p A^{A A}:: g f p$.

qRT-PCR and western hybridization experiments were performed to analyze wether the point mutations in $u s p A^{A A}:$ :gfp affect $u s p A$ gene transcription or translation (Figure 27). Mycelia of wild type strain, uspA::gfp or $u s p A^{A A}:$ :gfp expressing strains were harvested after $20 \mathrm{~h}$ of growth in liquid cultures. After preparation of RNA and cDNA synthesis, qRT-PCRs were performed (Figure 27A). uspA::gfp and $u s p A^{A A}:$ gfp reveal two to three fold higher expression of the uspA gene compared to wild type expression levels (Figure 27A). Furthermore, protein levels of the fusion proteins were analyzed with western hybridization experiments (Figure 27B). Quantification of the pixel density of the fusion protein band (red arrow) against the Ponceau $S$ loading control revealed no significantly different fusion protein amounts.

Cellular protein ubiquitination levels during fungal development were analyzed in $A$. nidulans wild type, uspA deletion and complementation strains as well as in the strain expressing $u s p A^{A A}:$ :gfp (Figure 28). Therefore, proteins were isolated after $20 \mathrm{~h}$ of vegetative growth in submerged culture, $24 \mathrm{~h}$ growth on solid agar plates in light to induce asexual development or $48 \mathrm{~h}$ of growth on agar plates in darkness with limited oxygen supply to induce sexual development. The amount of proteins modified with ubiquitin was analyzed by western hybridization using a aUbiquitin antibody. Deletion of uspA results in increased amounts of ubiquitinated proteins compared to the wild type at all tested growth states (Figure 28). 
A

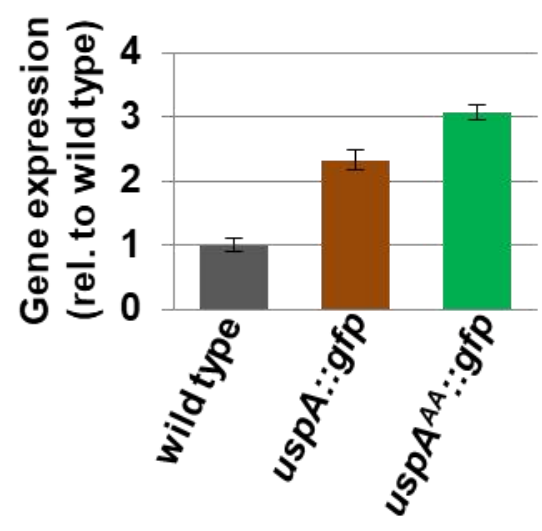

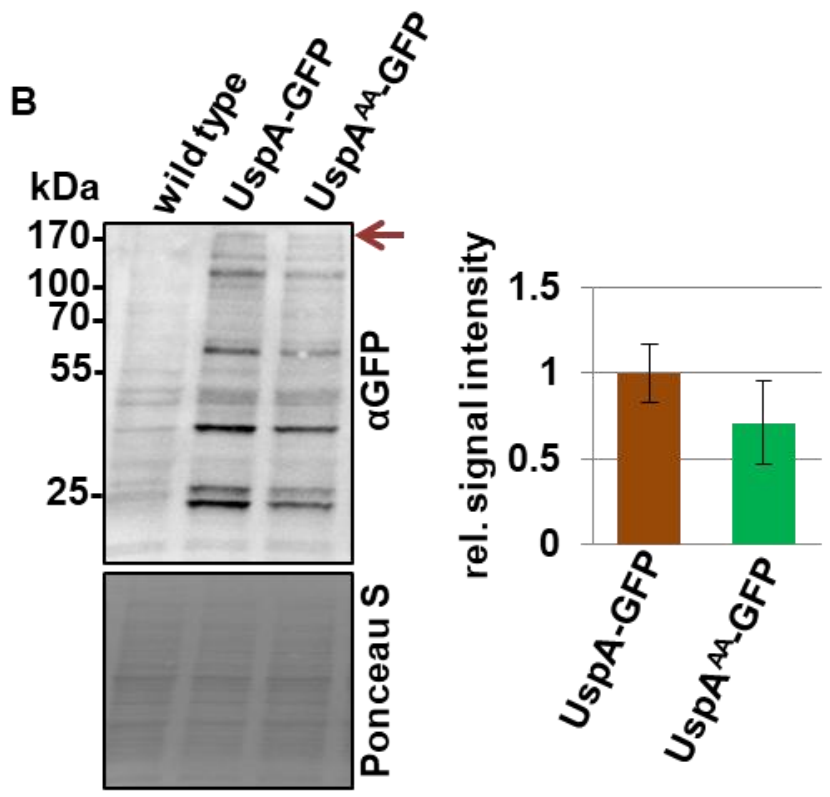

Figure 27: The fusion protein UspA ${ }^{\mathrm{AA}}$-GFP is transcribed and expressed like UspA-GFP.

A) Mycelia derived from $20 \mathrm{~h}$ vegetatively grown cultures were used for RNA extraction and subsequent cDNA synthesis. Wild type expression level of uspA was set to 1. uspA::gfp and $u s p A^{A A}::$ gfp reveal two to three fold elevated expression than the wild type. $h 2 A$ and $15 S$ rRNA served as housekeeping genes. Results derive from two independent biological replicates with three technical replicates each. Error bars represent the standard error of the mean (SEM). B) Western hybridization experiments of total protein crude extracts derived from vegetatively grown cultures were performed. Wild type crude extract was included as control to estimate the unspecific binding of the aGFP antibody. Signals of the fusion protein band (highlighted with red arrow) were quantified using Ponceau S staining as loading control. Error bars represent the SEM of two biological replicates.

This effect was independent from the utilized marker cassette. Ectopic integration of the uspA ORF into the genome of the deletion strain (comp $p^{\text {pyroA }}$ ) as well as in locus complementation (uspA::gfp) restored the amount of ubiquitinated proteins to wild type level. The strain expressing UspA ${ }^{\text {AA }}$-GFP shows an increase in the total amount of ubiquitinated proteins as well. The amount of modified proteins in the strain expressing the inactive UspA-GFP mutant is similar to both deletion strains. This indicates that the two point mutations render the UspA deubiquitinating enzyme inactive. The highest accumulation of ubiquitinated proteins was detected during asexual development in $u s p A$ deletion or inactive mutant strains. The level of proteins modified with ubiquitin is in these strains more than 2.5 times higher compared to the wild type and complementation strains.

This shows that UspA is a major deubiquitinase of $A$. nidulans, which is active throughout the whole fungal development. The two cysteine residues, one is part of the catalytic triad and the 
other one is a member of the zinc finger motif, seem to be essential for the deubiquitination activity. UspA-GFP without these amino acid substitutions showed wild type like levels of ubiquitinated proteins.

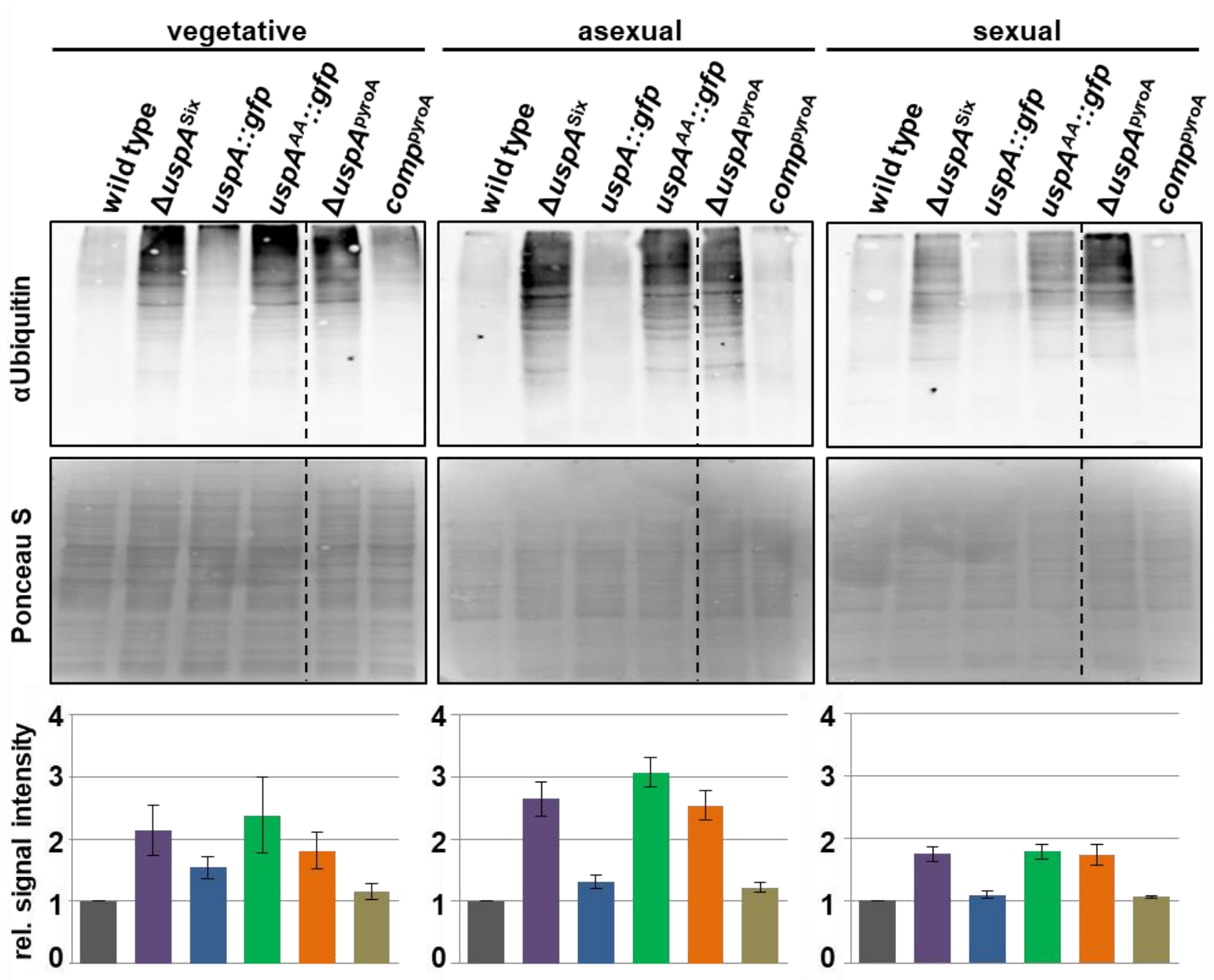

Figure 28: UspA deubiquitinates proteins during fungal growth and development.

Western hybridization of total protein crude extracts derived from mycelia grown for $20 \mathrm{~h}$ vegetatively in submerged cultures. For asexual and sexual development, mycelia were shifted from liquid cultures on solid agar plates and incubated for $24 \mathrm{~h}$ in light or darkness to induce respective development. Nitrocellulose membranes were incubated with aUbiquitin antibody. Signals were normalized to the loading control (Ponceau S). Error bars represent the standard error of the mean (SEM) of at least two biological repetitions. Wild type ubiquitination levels were set to 1 . The dashed lines indicate that the samples are not on the same membrane. The respective control strains used for quantification were loaded on all membranes. 


\subsection{UspA interacts with proteins involved in nuclear transport, RNA processing and the ubiquitin-proteasome system}

GFP pull down experiments were performed to identify interaction partners of UspA including putative substrates for deubiquitination. Due to the velocity of catalytic reactions, like deubiquitination processes, GFP pull downs were additionally performed with the UspA ${ }^{\mathrm{AA}}$-GFP mutant protein. The mutated protein might have an increased binding affinity to its substrates than the functional UspA-GFP fusion protein as it cannot catalyze the enzymatic reaction. A strain overexpressing free GFP served as negative control. GFP pull down experiments were performed with crude cell extracts of cultures grown for $20 \mathrm{~h}$ under submerged conditions. The functional as well as the mutant fusion protein could be identified in the elution fractions of the GFP pull downs using western hybridization experiments with a aGFP antibody (Figure 29).

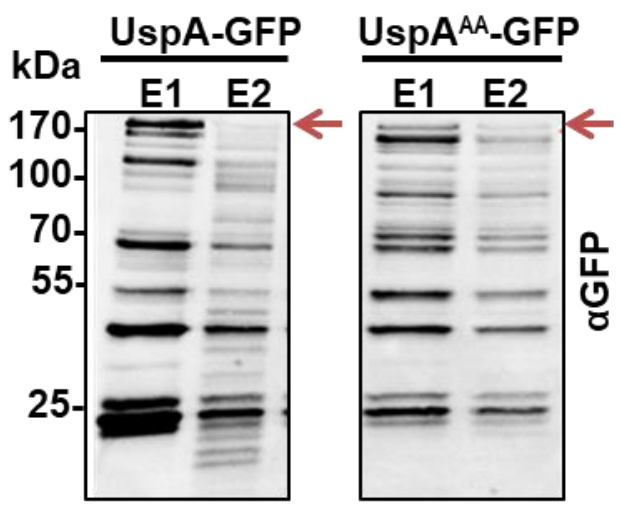

Figure 29: UspA-GFP and Usp $A^{\mathrm{AA}}$-GFP were enriched in GFP pull down experiments. Western hybridization experiments were performed with aliquots of different GFP pull down elution fractions (E1, E2). Signals for UspA-GFP as well as for UspA AA-GFP fusion protein were observed. Protein concentrations were higher in the first elution (E1) than in the second (E2). The red arrow marks the respective UspA-GFP fusion protein signal, degradation products and unspecific signals are visible below.

Protein compositions of the elution fractions were analyzed with LC/MS-MS. Proteins that were identified in at least two out of three biological replicates with a $\log _{2} L F Q$ intensity equal to or greater than 21 and that were not identified in the GFP control were further considered for data analysis. In total, 59 proteins were used for further data analysis (Figure 30, Tables 14-16). Among them, 38 proteins were pulled by both fusion proteins: UspA-GFP and UspA ${ }^{\mathrm{AA}}$-GFP (Table 14, Figure 30). Furthermore, 14 proteins were only pulled down by UspA ${ }^{\text {AA }}$-GFP (Table 15, Figure 30 ), whereas seven proteins were only identified in the pull downs of the functional UspA-GFP (Table 16, Figure 30). 


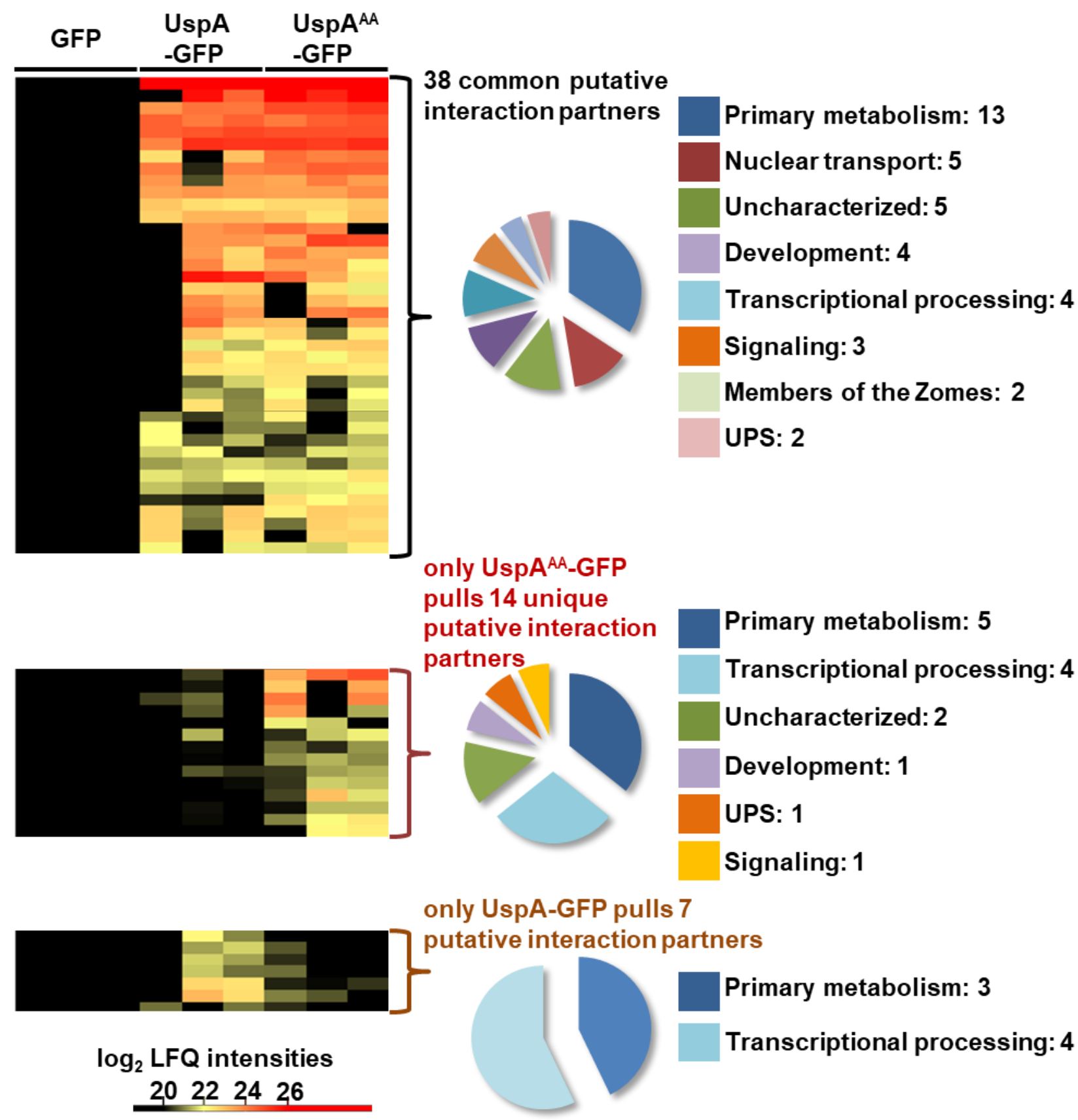

Figure 30: Identification of putative interaction partners of UspA-GFP and UspA ${ }^{\mathrm{AA}}$-GFP with LC/MS-MS.

The heat map displays the $\log _{2}$ label free quantification (LFQ) intensities of proteins identified in GFP pull down experiments. The results of three independent experiments of GFP control, UspA-GFP and UspA ${ }^{\mathrm{AA}}$-GFP are shown. The identified proteins were assigned to different categories that are illustrated as pie charts. The numbers represent the amount of proteins assigned to the different classes. Data analysis was performed with the MaxQuant and Perseus software. 
The bait protein UspA was identified with highest intensity values and highest amount of unique peptides in pull downs with both fusion proteins. Among the 38 proteins, which were identified in the pull downs of both protein variants, one third was assigned to the group of primary metabolism (Table 14). Two members of the Zomes were identified. The Zomes comprise the three structural very similar protein complexes COP9 signalosome, the proteasomal LID and the eukaryotic initiation factor 3 (elF3) (Pick and Pintard, 2009). Both UspA versions pulled one subunit of the proteasomal LID, RpnF, and one subunit of elF3, elF3D. Furthermore, five proteins related to nuclear transport, including the karyopherins KapB (AN0906) and KapF (AN6734) were pulled. Proteins related to transcriptional processing and fungal development were also detected.

Table 14: Functional groups of proteins identified with LC/MS-MS in pull downs of UspAGFP and UspA ${ }^{\mathrm{AA}}$-GFP.

Description of identified proteins derive from information on AspGD or FungiDB (Cerqueira et al., 2014; Stajich et al., 2012). Domain predictions for uncharacterized proteins were performed with NCBI CD domain prediction tool (Marchler-Bauer et al., 2015).

\begin{tabular}{|l|l|}
\hline $\begin{array}{c}\text { Systematic } \\
\text { Name }\end{array}$ & \multicolumn{1}{c|}{ Pescription } \\
\hline \multicolumn{2}{|c|}{ Primary metabolism } \\
\hline AN3524 & NAD binding Rossmann fold oxidoreductase \\
\hline AN7199 & Uncharacterized protein, galactonate metabolism \\
\hline AN0723 & Uncharacterized protein, domains of sulfotransfer superfamily \\
\hline AN7590 & Uncharacterized protein, mannitol dehydrogenase \\
\hline AN1318 & Uncharacterized protein, tyrosinase \\
\hline AN6521 & Homoaconitase, mitochondrial \\
\hline AN4956 & Acetolactate synthase \\
\hline AN8782 & Esterase \\
\hline AN6952 & Uncharacterized protein, S-adenosyl dependent methyltransferase \\
\hline AN7895 & Zinc-binding alcohol dehydrogenase domain-containing protein, CipB \\
\hline AN1023 & Actin cytoskeleton-regulatory complex protein, End3 \\
\hline AN7111 & Multifunctional beta-oxidation protein, FoxA \\
\hline AN5311 & Tyrosinase \\
\hline AN7334 & Uncharacterized protein, metabolic processes \\
\hline & \\
\hline AN0906 & KapB \\
\hline AN3877 & Uncharacterized, domains of NTF2 like superfamily \\
\hline AN6734 & KapF \\
\hline AN5376 & Uncharacterized, domains of NTF2 like superfamily \\
\hline
\end{tabular}


Table 14: continued.

\begin{tabular}{|l|l|}
\hline $\begin{array}{c}\text { Systematic } \\
\text { Name }\end{array}$ & \multicolumn{1}{c|}{ Nuclear transport } \\
\hline \multicolumn{2}{|c|}{ Development } \\
\hline AN6978 & RCC1, chromatin associated guanine nucleotide exchange factor for Ran \\
\hline \multicolumn{2}{|c|}{ Transcriptional processing } \\
\hline AN10311 & Cell wall mannoprotein, MnpA \\
\hline AN5635 & Neutral trehalase \\
\hline AN2523 & Chitin synthase B \\
\hline AN6709 & Guanyl-nucleotide exchange factor (Sec7), hyphal morphogenesis \\
\hline \multicolumn{2}{|c|}{ Ubiquitin-proteasome system } \\
\hline AN5452 & Pre-mRNA-splicing factor, Rse1 \\
\hline AN1205 & Prefoldin subunit 5, regulation of transcriptional elongation \\
\hline AN5894 & Pol II transcription elongation factor subunit Cdc73 \\
\hline \multicolumn{2}{|c|}{} \\
\hline AN6354 & Ubiquitin carboxyl-terminal hydrolase, UspA \\
\hline AN7422 & Ubiquitin carboxyl-terminal hydrolase \\
\hline \multicolumn{2}{|c|}{ Zomes } \\
\hline AN7540 & Eukaryotic translation initiation factor 3 subunit D, elF3D \\
\hline AN10519 & Proteasome regulatory particle subunit, RpnF \\
\hline \multicolumn{2}{|c|}{ Signaling } \\
\hline AN1545 & Protein phosphatase PP2A regulatory subunit B \\
\hline AN12477 & Uncharacterized protein, GTPase activity \\
\hline AN10691 & Dynamin GTPase \\
\hline & Uncharacterized proteins \\
\hline AN0860 & Uncharacterized protein \\
\hline AN3121 & Uncharacterized, conserved glutamic acid-rich protein \\
\hline AN10518 & Uncharacterized protein \\
\hline AN2647 & Uncharacterized protein \\
\hline AN3709 & Uncharacterized protein, CRAL/TRIO domain protein \\
\hline
\end{tabular}

The putative USP AN7422 was coenriched with UspA. This protein was found in BLAST analyses of human Usp15 against the $A$. nidulans database (Table 13). qRT-PCRs were performed to investigate if the transcript levels are similarly upregulated in $\triangle c s n E$ as for uspA and the other putative USPs (Figure 21, Figure 31). The transcript levels of AN7422 were upregulated during multicellular fungal development, but not during vegetative growth, in the 
absence of CsnE. This is similar to the gene expression profiles of the other ubiquitin-specific protease encoding genes in a $\Delta c s n E$ strain (Figure 21).

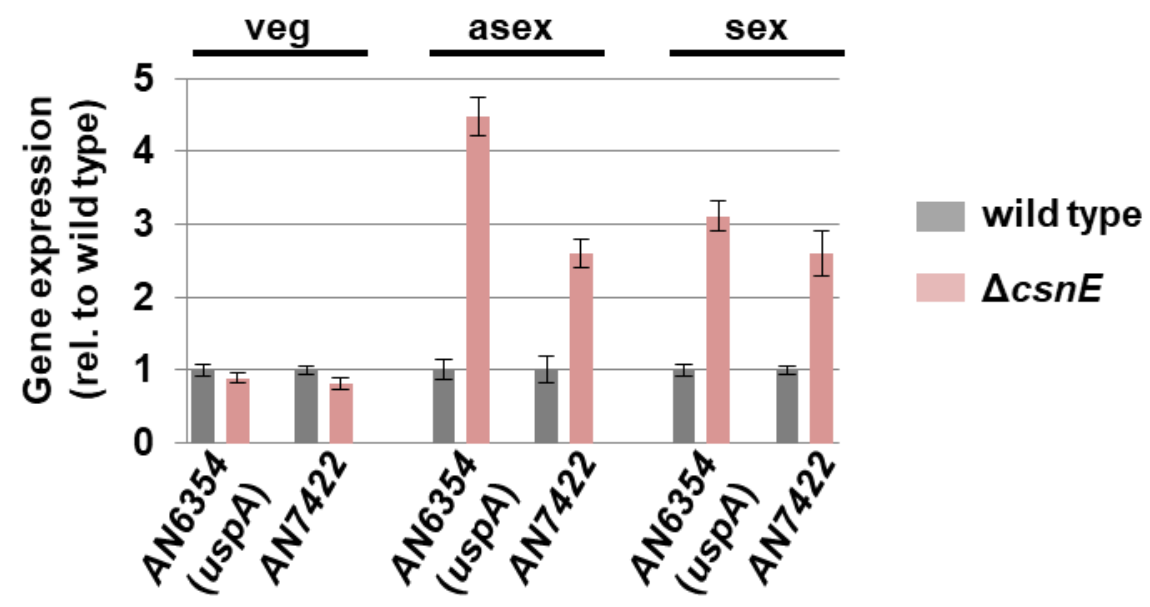

Figure 31: AN7422 transcript levels are upregulated in the absence of functional COP9 signalosome similar to uspA.

Expression levels of AN6354 (uspA) and AN7422 were analyzed using qRT-PCR. RNA derived from mycelia grown for $20 \mathrm{~h}$ in submerged culture (veg), from asexually (asex) or sexually (sex) grown mycelia were used. As reference genes served $h 2 A$ and $15 S r R N A$. Wild type expression was set to 1. Error bars represent standard error of the mean (SEM) of at least two biological replicates.

UspA ${ }^{\text {AA }}$-GFP pull downs were performed to identify substrates of the deubiquitinase. 14 proteins were identified in at least two out of three biological replicates only in the strain expressing the UspA $^{\text {AA }}$-GFP mutant (Table 15). UspA ${ }^{A A}$-GFP might bind longer to its putative substrates as it cannot catalyze the deubiquitination reaction. Identification of polyubiquitin in the pull downs with the mutated UspA-GFP version suggests that UspA does not bind to its substrates directly, but associates to their polyubiquitin chains.

Most proteins specifically pulled down by the inactive UspA-GFP mutant were assigned to primary metabolism, but also four proteins related to transcriptional processing were identified (Table 15). PhiA, a protein involved in fungal asexual development and a serine/threonine kinase were pulled down as well. UspA-GFP, but not UspA ${ }^{A A}$-GFP, pulled four more proteins categorized to transcriptional processing and three classified to primary metabolism (Figure 30, Table 16). 
Table 15: Proteins that were identified only in the pull down of UspA ${ }^{A A}-G F P$, but not in the pull down with functional UspA-GFP fusion protein.

Description of identified proteins derive from information on AspGD or FungiDB (Cerqueira et al., 2014; Stajich et al., 2012). Domain predictions for uncharacterized proteins were performed with NCBI CD domain prediction tool (Marchler-Bauer et al., 2015).

\begin{tabular}{|l|l|}
\hline $\begin{array}{c}\text { Systematic } \\
\text { Name }\end{array}$ & \multicolumn{1}{c|}{ Primary metabolism } \\
\hline \multicolumn{3}{|c|}{ Description } \\
\hline AN3331 & Uncharacterized protein, phosphohydrolase superfamily \\
\hline AN5328 & GPI anchored dioxygenase \\
\hline AN3616 & Uncharacterized protein, monooxygenase \\
\hline AN1689 & Aldehyde dehydrogenase family \\
\hline AN1882, & NADH-dependent flavin oxidoreductase \\
\hline AN1602 & Endo-beta-1,4-glucanase D \\
\hline \multicolumn{3}{|c|}{ Transcriptional processing } \\
\hline AN7680 & $\begin{array}{l}\text { Uncharacterized protein, SMC superfamily, structural maintenance of } \\
\text { chromosomes }\end{array}$ \\
\hline AN0646 & DNA/RNA helicase activity \\
\hline AN2007 & Small nuclear ribonuceloprotein SmD3, mRNA binding \\
\hline AN4965 & Ccr4-Not transcription complex subunit \\
\hline \multicolumn{3}{|c|}{ Development } \\
\hline AN8333 & PhiA protein, phialide development \\
\hline & Unchiquitin-proteasome system \\
\hline AN0200 & Polyubiquitin \\
\hline & Serine/threonine kinase, PhoB \\
\hline AN1867 & Uncharacterized protein \\
\hline
\end{tabular}

The active and the inactive UspA pull 21 proteins related to fungal primary metabolism. Furthermore, UspA associates with eight proteins classified to the nuclear transport category and additionally seven proteins related to transcriptional processing. This suggests a function of the deubiquitinase in nuclear transport of proteins by deubiquitination reactions. The cellular localization of the fusion protein mainly close to nuclei with subpopulations in the nucleus or in the cytoplasm corroborates this hypothesis (Figure 25). Furthermore, the karyopherins might regulate the subcellular localization of UspA itself. Thereby, UspA might function at the 
interphase of cytoplasmic and nuclear transport of proteins or mRNAs, which might be regulated through its deubiquitination acitvity. The specific task of UspA might thereby be dependent on its localization, which in turn could be determined through the karyopherins.

Table 16: Proteins exclusively identified in UspA-GFP pull down.

Description of identified proteins derive from information on AspGD or FungiDB (Cerqueira et al., 2014; Stajich et al., 2012). Domain predictions for uncharacterized proteins were performed with NCBI CD domain prediction tool (Marchler-Bauer et al., 2015).

\begin{tabular}{|l|l|}
\hline $\begin{array}{c}\text { Systematic } \\
\text { Name }\end{array}$ & \multicolumn{1}{c|}{ Description } \\
\hline \multicolumn{2}{|c|}{ Primary metabolism } \\
\hline AN2947 & 1-phosphatidylinositol-4,5-bisphosphate phosphodiesterase \\
\hline AN5883 & Methylenetetrahydrofolate reductase, MetF \\
\hline AN2493 & Extracellular phytase, gluconate metabolism \\
\hline \multicolumn{2}{|c|}{ Transcriptional processing } \\
\hline AN03955 & Uncharacterized protein, orthologs function in RNA metabolic process \\
\hline AN11128 & RNA polymerase II transcription elongation factor, Ctr9 \\
\hline AN7480 & Differentiation regulator, Nrd1, RNA binding \\
\hline AN4024 & RNA maintenance of telomere capping protein 1 \\
\hline
\end{tabular}

\subsection{UspA ensures coordinated fungal development and secondary metabolism}

\subsubsection{UspA is required for asexual spore formation}

The increased amount of ubiquitinated proteins in fungal total protein crude extracts especially during asexual development and the upregulation of $u s p A$ transcript levels in $\triangle \operatorname{csn} E$ indicate a role of the deubiquitinase in fungal development. Asexual development of $A$. nidulans wild type, usp $A$ deletions $\left(\Delta u s p A^{p y r o A}, \Delta u s p A^{\text {Six }}\right)$, complementation strains (comp pyroA, uspA::gfp) and the inactive mutant strain expressing $u s p A^{A A}:$ :gfp was investigated (Figure 32). All strains form colonies of similar size. The uspA deletion strains as well as the strain expressing the inactive mutant form whitish airial mycelia in the middle of the colony. Furthermore, the rather light green color of the colony when compared to the wild type and complementation strains suggests a reduced formation of asexual conidiospores (Figure 32). The bottom of the wild type and complementation colonies are dark brown, whereas the deletion strains show brighter pigmentation, indicating altered secondary metabolism (Figure 32). 


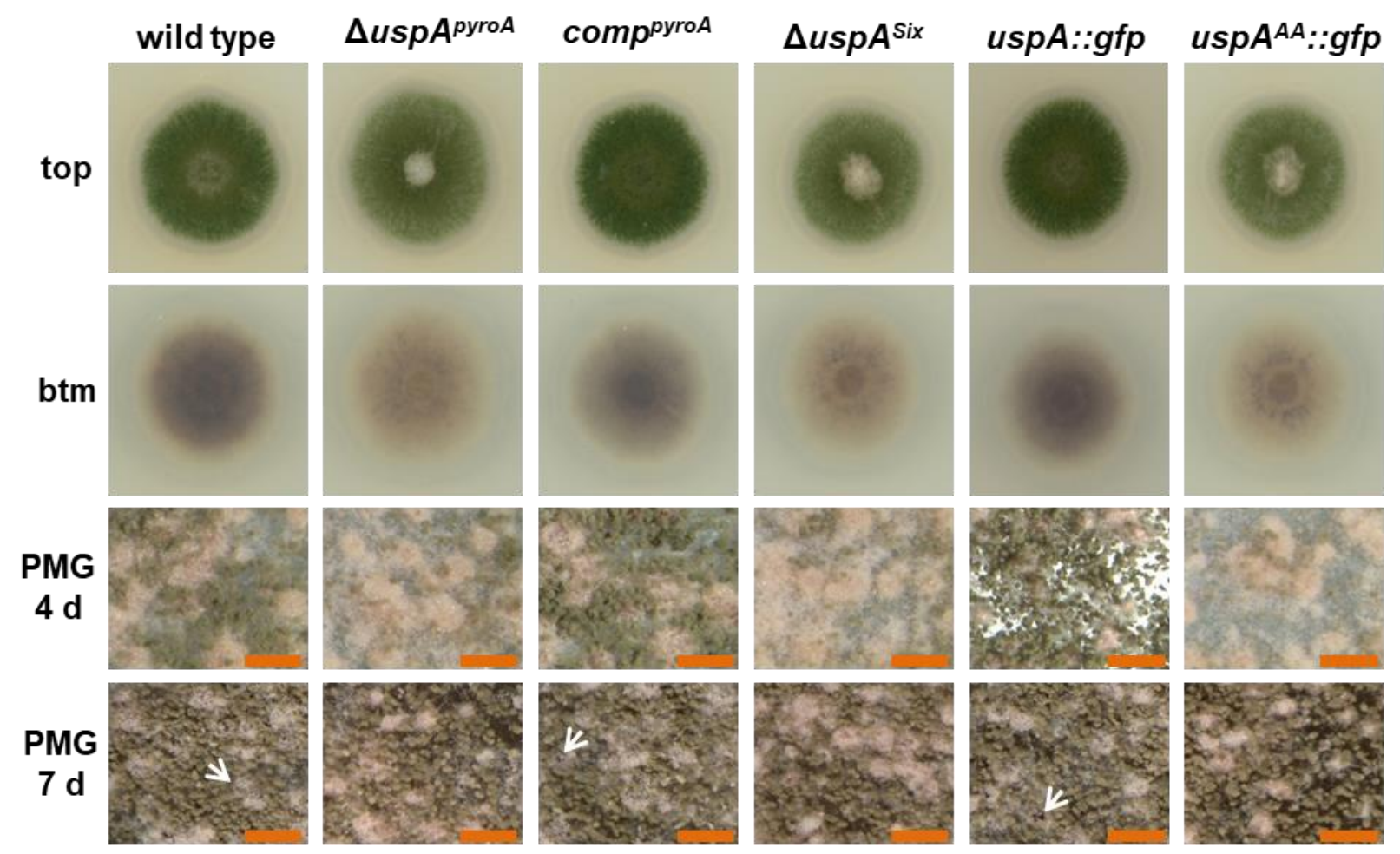

Figure 32: UspA induces conidiospore formation in A. nidulans.

5,000 spores were point-inoculated on solid minimal medium containing agar plates. Strains were grown for four days at $37^{\circ} \mathrm{C}$ in light. Top and bottom (btm) view of the colonies are shown. For photomicrographs (PMG) 30,000 spores were distributed evenly on an agar plate and incubated for four or seven days under asexual growth inducing conditions. Mature cleistothecia are highlighted with a white arrow. Orange size bars represent $200 \mu \mathrm{m}$.

Photomicrographs indicate a reduced conidiospore formation for uspA deletion and inactive mutant strains after four days of development. The wild type strain (AGB551) and therewith the background of all deletion and complementation strains contains the full ORF of veA (veA+). Strains with the $v e A+$ locus produce cleistothecia even under conditions that normally favor asexual development (Adams et al., 1998; Stinnett et al., 2007). Sexual fruiting bodies are still immature and fully surrounded by Hülle cells after four days of growth under asexual inducing conditions in all strains. Wild type and complementation strains produce mature cleistothecia after seven days of incubation in light, whereas fruiting bodies of the deletion strains and usp $A^{A A}:$ :gfp expressing strains are still immature (Figure 32, lower panel). 30,000 spores were distributed evenly on a minimal medium containing agar plate for quantification of conidiospores from wild type, $\Delta u s p A^{\text {pyroA }}$ and comp pyroA. 


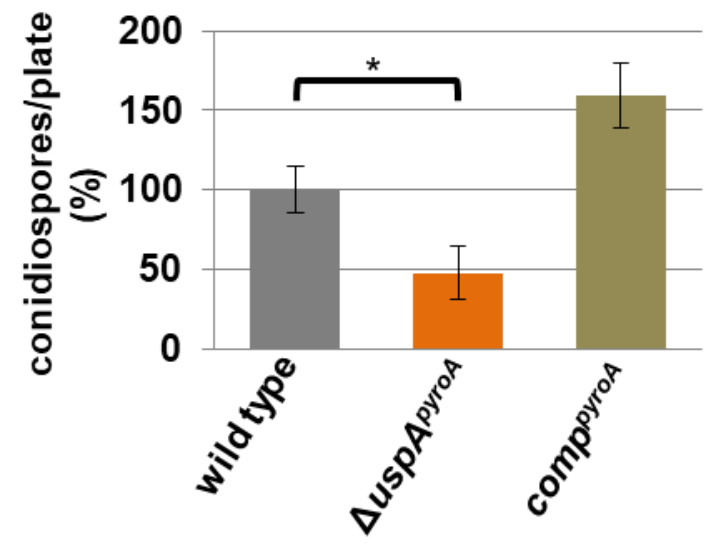

Figure 33: UspA is required for conidiospore formation.

30,000 spores were equally distributed on agar plates containing $30 \mathrm{ml}$ of minimal media. Conidiospores were harvested and counted after four days of growth during asexual development inducing conditions. Spore numbers derived from five biological replicates with two technical replicates each. Error bars represent standard error of the mean (SEM). P-value was calculated using standard deviation; * $\mathrm{p} \leq 0.05$.

This analysis showed that the $\triangle u s p A^{p y r o A}$ forms approximately half of the conidiospores compared to the wild type. The complementation strain, which restored the deletion phenotype by ectopical integration of the uspA ORF, forms about $50 \%$ more spores than the wild type strain (Figure 33). The conidiospore amount is correlating with the intensity of the green color of the colonies (Figure 32).

In the $\triangle u s p A^{p y r o A}$ deletion strain the original $u s p A$ ORF is replaced by the pyroA marker from A. fumigatus. Therefore, the deletion strain is not auxotrophic for pyridoxine anymore and does not need the supplementation in the media. Since the wild type used in this study is auxotrophic for pyridoxine, all experiments performed in this study were performed with minimal medium supplemented with pyridoxine. The respective complementation strain (comp proA) contains an ectopic integrated uspA ORF, which means that the pyroA marker is still present and the complementation does not require pyridoxine in the medium as well. The phenotype of uspA deletion strain on medium without pyridoxine is more pronounced than on medium with pyridoxine (Figure 34A). The colony diameter is smaller after four days of development compared to the wild type and the complementation strain. Photomicrographs show drastically reduced conidiospore formation after four and seven days of growth during asexual development (Figure 34A). 


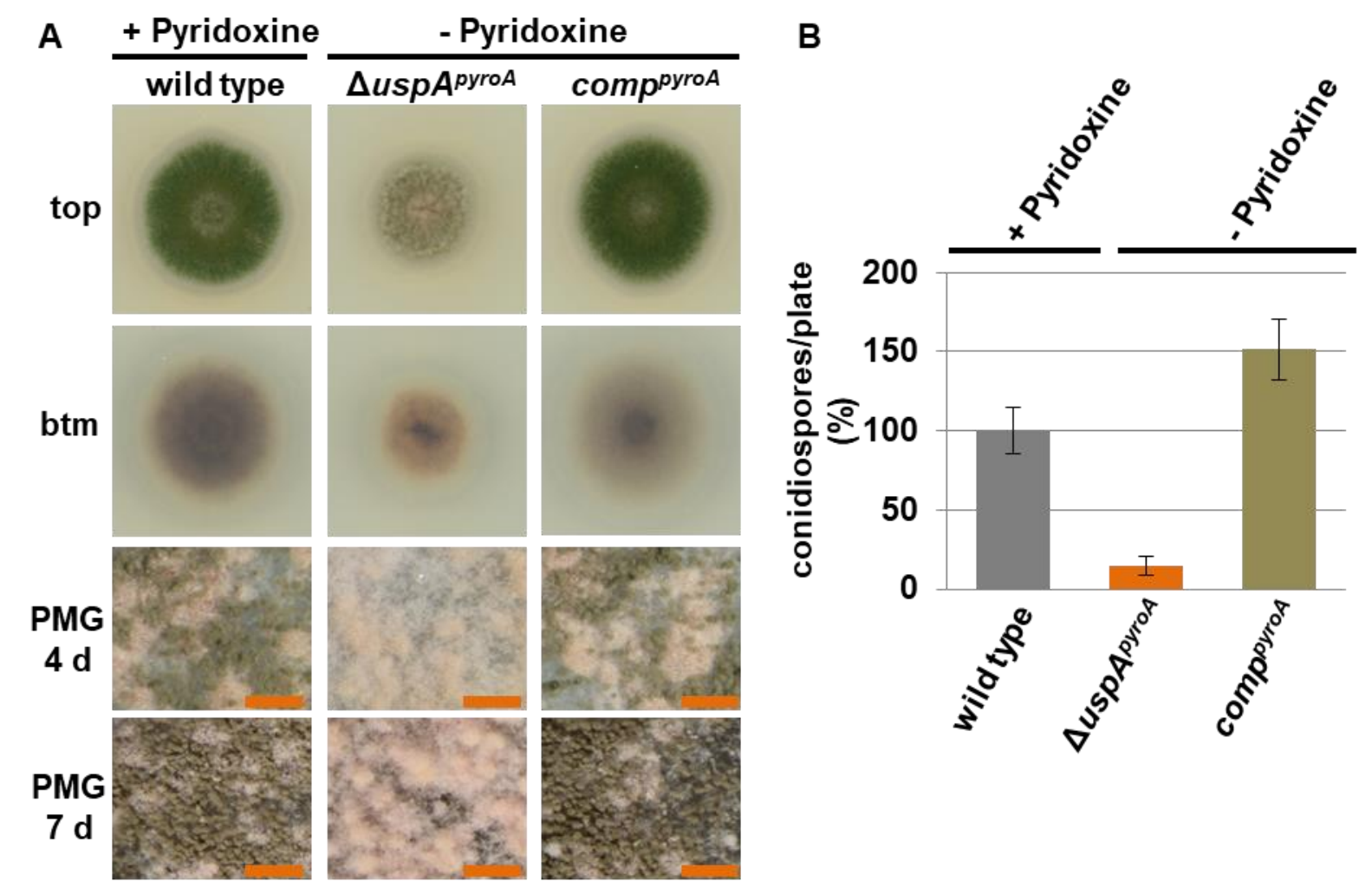

Figure 34: The lack of pyridoxine supplementation emphasizes the uspA deletion phenotype.

A) 5,000 spores were point-inoculated on medium with pyridoxine for the wild type strain and without pyridoxine for $\triangle u s p A^{p y r o A}$ and $c o m p^{p y r o A}$ strains. Strains were cultivated for four days under asexual development inducing conditions. For photomicrographs (PMG) 30,000 spores were distributed evenly on agar plates with respective medium and incubated for four or seven days. Orange size bar represents $200 \mu \mathrm{m}$. B) Conidiospore quantification was done of these strains after four days of growth on respective medium. Numbers derive from five biological replicates with at least two technical replicates, respectively. Error bars represent the standard error of the mean (SEM).

Quantification of conidiospores revealed only $15 \%$ of the amount of wild type conidiospores in $\triangle u s p A^{\text {pyroA }}$ strain on medium without pyridoxine. The complementation, which still carries the pyroA marker cassette, complements the phenotype completely, even producing more conidiospores than the wild type strain on medium lacking pyridoxine. 


\subsubsection{UspA accelerates sexual development}

A. nidulans enters the sexual life cycle and forms mature cleistothecia after seven days of development in darkness and under oxygen limiting conditions. The formation of cleistothecia, which serve the fungus as overwintering structure and to survive harsh environmental conditions, requires a lot of energy (Axelrod et al., 1973). Fungal mutant strains lacking a functional COP9 signalosome are not able to produce mature sexual fruiting bodies. The development is blocked at the state of immature primordia (Beckmann et al., 2015; Busch et al., 2007). Here, it was shown that the transcript levels of uspA and also other ubiquitin-specific protease encoding genes are increased in the absence of a functional COP9 signalosome. The sexual development and cleistothecia formation was investigated in uspA deletion strains $\left(u s p A^{p y r o A}, u s p A^{S i x}\right)$, complementation strains (comp pyroA, usp $\left.A:: g f p\right)$ and the strain expressing the inactive $u s p A^{A A}:$ gfp mutant (Figure 35A). All strains revealed a similar colony radius, indicating similar growth rates. The bottom of the colony of $u s p A$ deletion and mutant strains show a rather reddish color compared to wild type and complementation strains, indicating an altered secondary metabolism (Figure 35A).

The colonies show the formation of different circular regions: the inner region (ir), the middle region (mr) and the outer region (or). The middle circle consists of the oldest mycelium in the point-inoculated fungal colonies, whereas the outer most region represent the youngest mycelium of the colony. Therefore, different aged mycelia can be observed in these colonies. The inner region has a dark green color and consists of a mixture of conidiospores and mature cleistothecia. The middle region is represented by a brighter green color. It consists of mature cleistothecia, which are surrounded by nursing Hülle cells and some conidiospores. This radius of the middle region is smaller in $u s p A$ deletion or mutant strains compared to wild type and complementation strains (Figure 35B). Therefore, the radius of the outer region with the yellow color is larger in strains that do not carry a functional deubiquitinase compared to wild type and complementation strains. The outer region contains immature sexual fruiting bodies, which are covered with many Hülle cells that confer the yellow color (Figure 35B). The age of the growing mycelium is decreasing from the inner to the outer region. Differences in colony formation were observed in the middle and the outer circular regions in wild type and complementation strains compared to deletion and non-functional mutant strains. This suggests that UspA might be necessary for early steps of sexual development. 


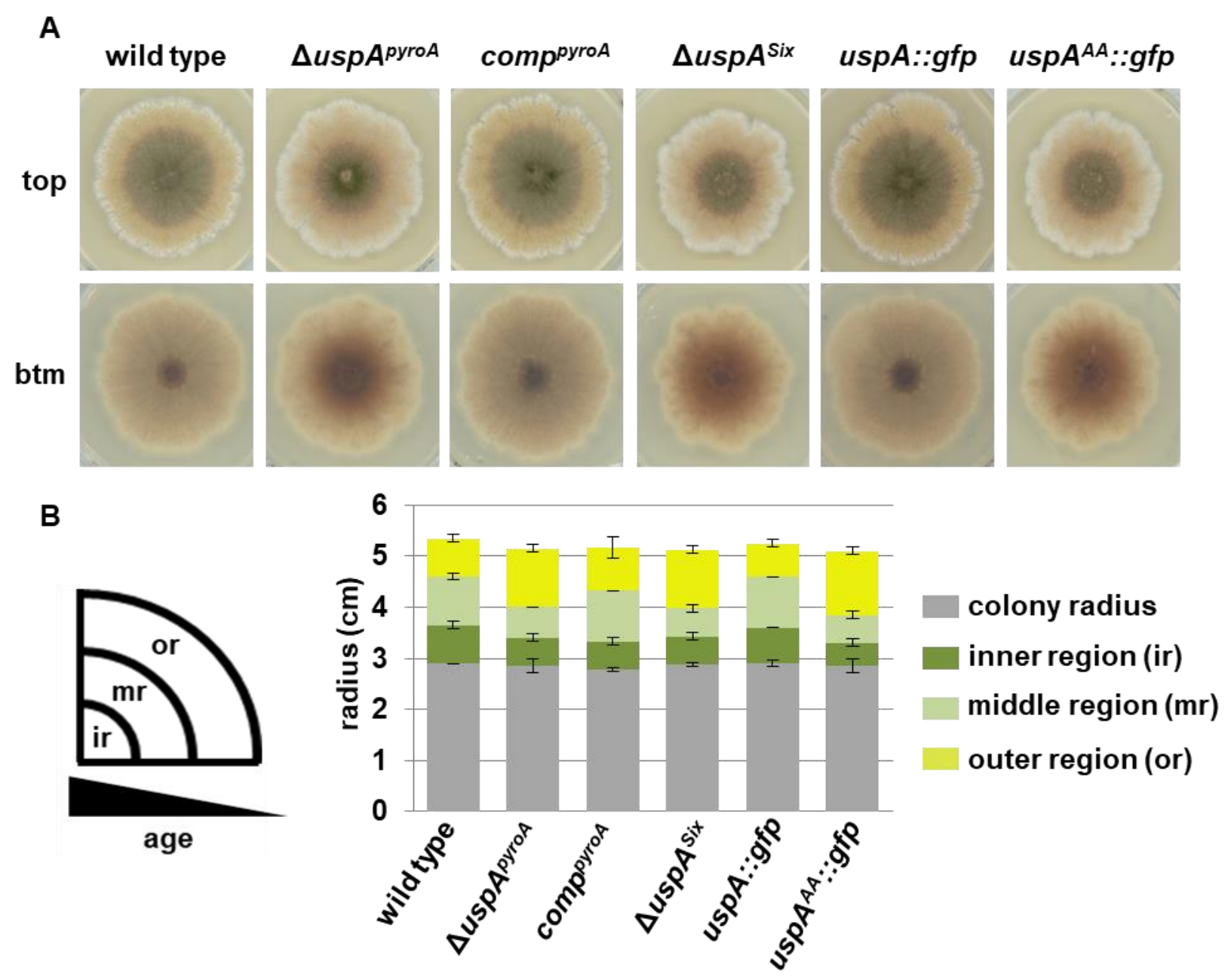

Figure 35: UspA is required for the early steps of cleistothecia development.

A) 5,000 spores of wild type, uspA deletion and complementation strains as well as the usp $A^{A A}:$ :gfp mutant strain were point-inoculated on minimal medium containing pyridoxine. Agar plates were incubated for seven days at $37^{\circ} \mathrm{C}$ in the dark and under oxygen limiting conditions. The top and the bottom (btm) of the colonies are shown. B) Radius of the circular regions observed in A). A schematic representation of the colony illustrates the inner region (ir), middle region (mr) or outer region (or). The age of the mycelia decreases in the direction of the edges of the colony. The radius of the whole colony and "ir" is similar in all strains. The radius of the "mr" and the yellow "or" differ in strains harboring a functional compared to a non-functional UspA. Error bars represent standard deviations (SD) of two independent biological replicates.

Sexual fruiting body formation was analyzed after three, five and seven days of growth under sexual development inducing conditions to analyze the effect of UspA on fruiting body development (Figure 36). All strains showed the formation of early nests after three days of growth under sexual development inducing conditions, which are completely covered by Hülle cells. Mature cleistothecia are already formed in wild type and complementation strains after five days of growth. The uspA deletion and inactive mutant strains show development of 
cleistothecia as well. Nevertheless, they are still surrounded by more Hülle cells and fruiting bodies seem to be smaller and not as mature as the ones from wild type and complementation strains. After seven days of growth, uspA deficient strains overcome the delay in development and form cleistothecia that contain ascospores.

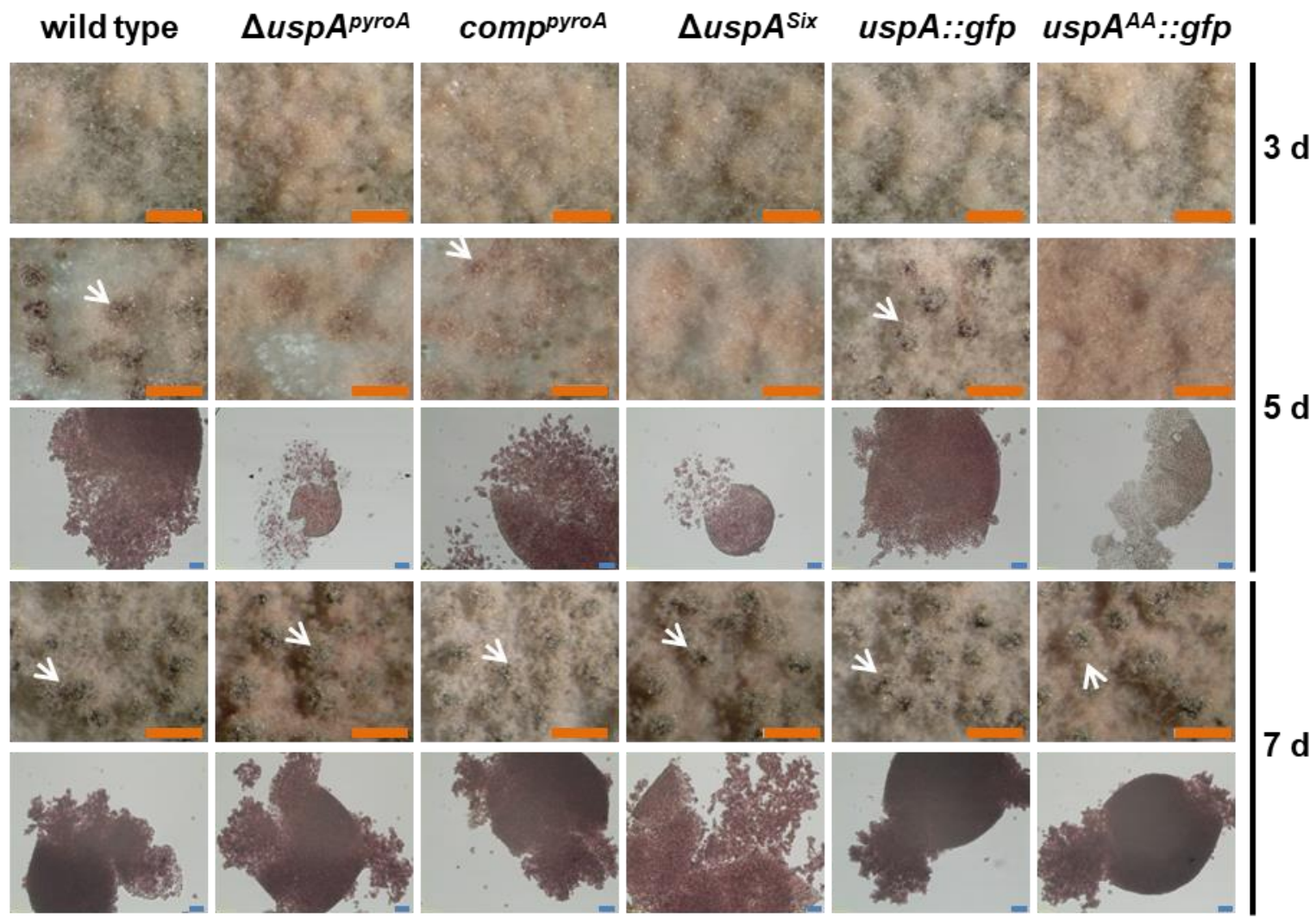

Figure 36: UspA is important for correct timing in early sexual development.

30,000 spores were inoculated on agar plates containing minimal media and incubated for three, five or seven days at $37^{\circ} \mathrm{C}$ in the dark under oxygen limiting conditions. Cleistothecia formation was observed at the indicated time points. White arrows indicate mature cleistothecia. Orange size bars represent $200 \mu \mathrm{m}$; blue size bars represent $50 \mu \mathrm{m}$.

Sexual development, similar to asexual development, is more affected in $\triangle u s p A^{p y r o A}$ when the medium is not supplemented with pyridoxine. The colony of $\Delta u s p A^{p y r o A}$ is much smaller than the colony of wild type and complementation strains (Figure 37). It also does not form the rings that represent developmental structures of different age. It does not develop conidiospores and consists of immature cleistothecia covered by Hülle cells that are responsible for the yellow color even after seven days of development. 


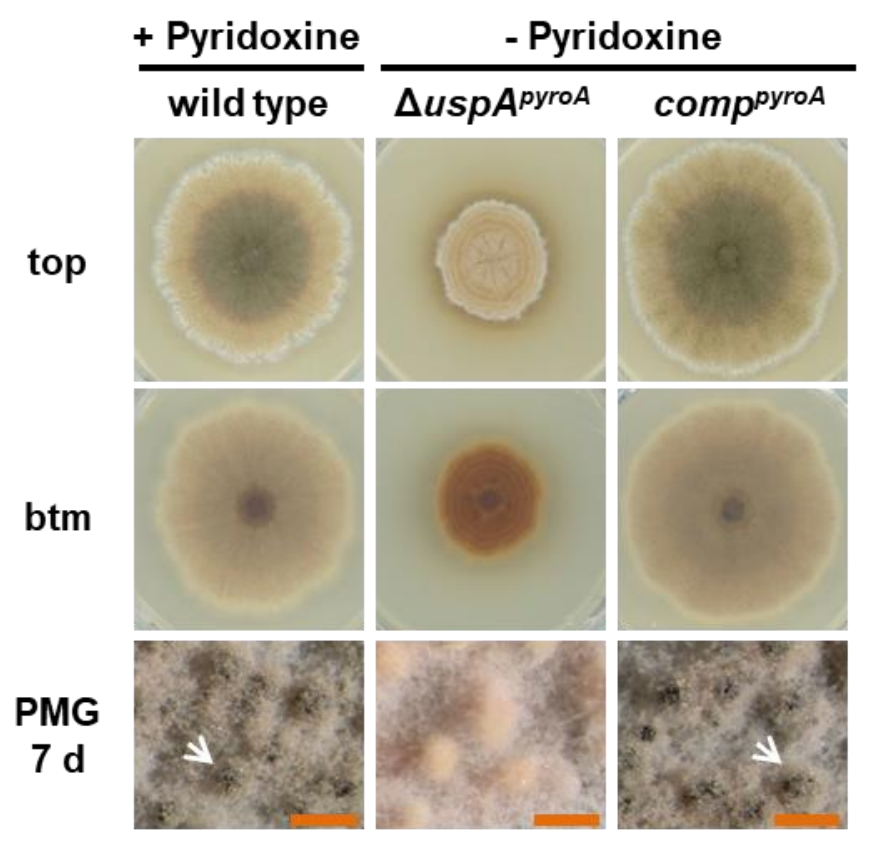

Figure 37: The lack of pyridoxine in the culture medium enhances the deletion phenotype. 5,000 spores were point-inoculated on minimal medium containing pyridoxine (for wild type) and on minimal medium without pyridoxine (for $\triangle u s p A^{\text {pyroA }}$ and $c o m p^{\text {pyroA }}$ ) and incubated for seven days under sexual development promoting conditions. For photomicrographs (PMG) 30,000 spores were distributed evenly on the agar plate containing respective media. White arrows highlight mature cleistothecia. Orange error bar represents $200 \mu \mathrm{m}$.

The bottom of the colony revealed a stronger red color than on medium supplemented with pyridoxine. Photomicrographs (PMG) of seven days old strains depict mature cleistothecia for the wild type strain. The complementation strain, which also grew on medium without pyridoxine, developed mature cleistothecia as well, whereas the deletion strain only formed early nests (Figure 37).

\subsubsection{UspA controls secondary metabolism}

Different pigmentation at the bottom of the colony of $\triangle u s p A$ compared to wild type and the complementation strain during multicellular development suggests altered secondary metabolism. Aspergillus nidulans mutant strains with defects in the ubiquitin-proteasome system are promising candidates to investigate secondary metabolite gene clusters that are silenced in wild type strains (Gerke et al., 2012). The derivative of benzaldehyde ( $\underline{d b a})$ gene cluster was identified in $\triangle c s n E$ (Gerke et al., 2012). The expression of the genes belonging to this cluster is silenced in wild type, whereas all genes are upregulated during at least one developmental 
stage in $\Delta c s n E$ (Gerke et al., 2012). Deubiquitinating enzymes are essential for a functional ubiquitin-proteasome system. Therefore, expression of the dba gene cluster in the wild type A. nidulans strain was investigated in comparison to $\Delta u s p A^{\text {pyroA }}$ and $c o m p^{\text {pyroA }}$ during multicellular fungal development. This analysis revealed an upregulation of several genes of the dba gene cluster in absence of UspA (Figure 38). $d b a D$, encoding a substrate transporter, shows the highest upregulation during asexual and sexual development in $\Delta u s p A^{\text {pyroA }}$ compared to wild type and complementation strain (Figure 38).

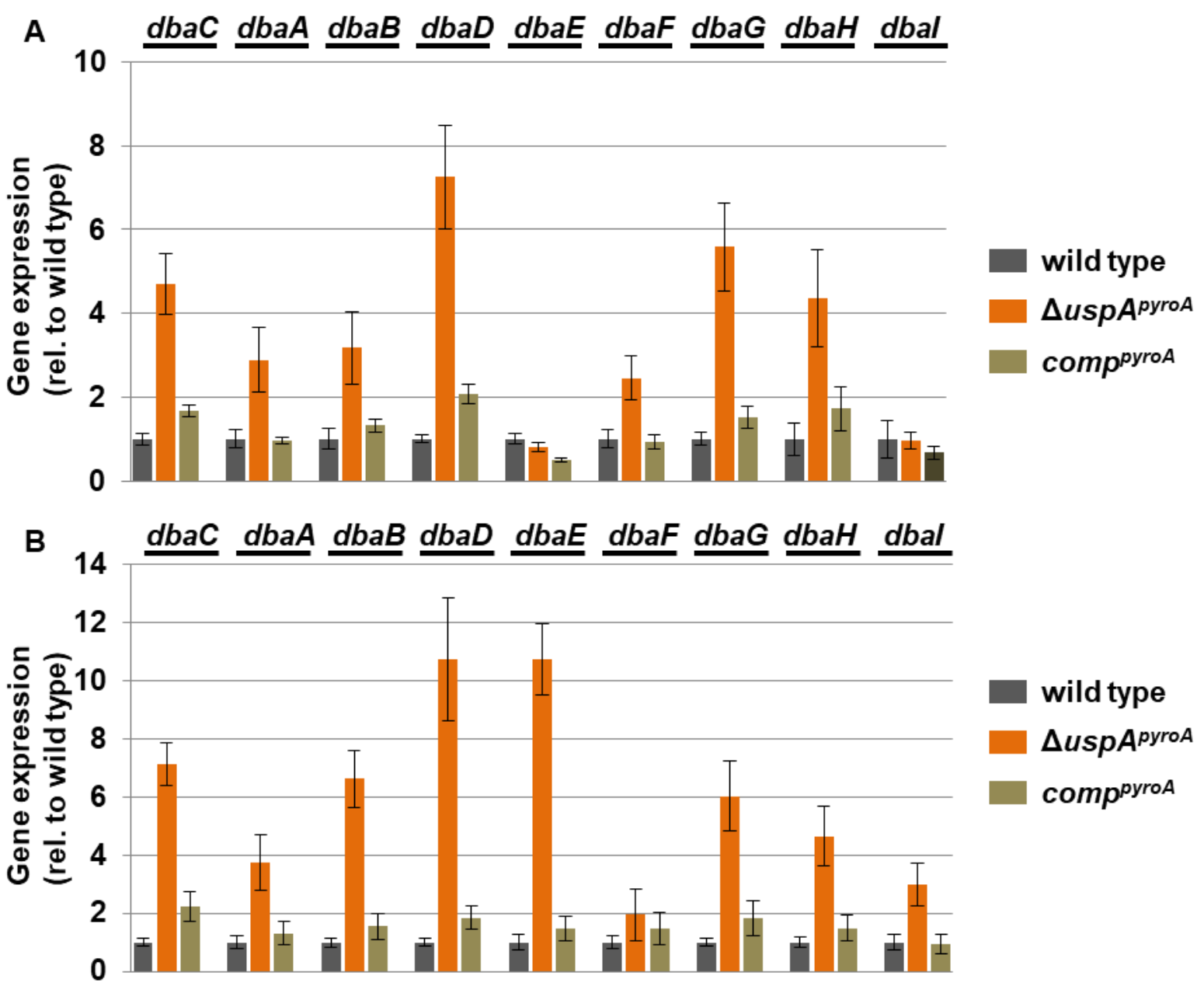

Figure 38: UspA causes repressed expression of genes of the derivative of benzaldehyde (dba) cluster during multicellular development.

qRT-PCRs were performed with cDNA derived from different developmental stages of $A$. nidulans wild type, uspA deletion $\left(\triangle u s p A^{p y r o A}\right)$ and respective complementation strain $\left(\right.$ comp $\left.p^{\text {pyroA }}\right)$. RNA was extracted and cDNA was synthesized from mycelia grown for $24 \mathrm{~h}$ under asexual development inducing conditions (A) or from mycelia grown for $48 \mathrm{~h}$ under sexual development inducing conditions (B). Gene expression was normalized against $h 2 A$ and 15S rRNA and wild type expression was set to 1. Error bars represent standard error of the mean (SEM) of three biological and three technical replicates, respectively. 
More than fourfold upregulation during asexual development showed $d b a C$, encoding an YCII domain protein, $d b a G$, encoding a putative transcription factor and $d b a H$, encoding a monooxygenase, in $\Delta u s p^{p y r o A}$ compared to wild type. Furthermore, upregulation were detected for $d b a A$, encoding a $\mathrm{Zn}(\mathrm{II})_{2}-\mathrm{Cys}_{6}$ transcription factor, $d b a B$, encoding a monooxygenase as well as for $d b a F$, encoding a FAD dependent oxidoreductase, in the uspA deletion strain. The gene encoding the esterase $(d b a E)$ and the polyketide synthase ( $d b a l)$ are not differently regulated in $\Delta u s p A^{p y r o A}$ compared to wild type during asexual development (Figure $38 \mathrm{~A}$ ). Genes encoding the substrate transporter $(d b a D)$ and the esterase $(d b a E)$ are ten times upregulated in $\Delta u s p A$ compared to wild type strain during sexual development. Most of the other genes of the dba cluster show upregulation in $\triangle u s p A^{p y r o A}$ compared to wild type as well. No significant difference in gene expression was observed for $d b a F$ encoding the FAD dependent oxidoreductase during sexual development inducing conditions (Figure 38B).

In summary, UspA is required for conidiospore development, early sexual fruiting body development and coordinated expression of the dba secondary metabolite gene cluster. The $\triangle u s p A^{p y r o A}$ deletion strain forms only $50 \%$ conidiospores on minimal medium supplemented with pyridoxine and less than $20 \%$ on medium lacking pyridoxine compared to the wild type conidiospore amount. UspA might have a supportive function in early stages of sexual development as cleistothecia development is retarded after five days compared to wild type. However, uspA deficient strains are able to produce mature cleistothecia. The $d b a$ gene cluster was discovered to be upregulated in $\triangle c s n E$, therefore CsnE leads to reduced expression of the dba gene cluster (Gerke et al., 2012). Here, it was shown that also UspA has a repressive effect on this gene cluster. This is another hint that both proteins act together in protein half-life control.

\subsection{UspA alters protein levels of the major fungal regulator VeA, but not of its interaction partner VelB during the initiation of multicellular development}

The human ortholog of UspA, which is Usp15, regulates the stability of the inhibitor of the NF-kB transcription factor protein family (Schweitzer et al., 2007). Members of this family are

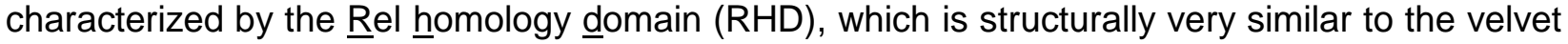
domain of the fungal velvet domain protein family (Ahmed et al., 2013). The most investigated member of this protein family is $\mathrm{VeA}$, which is a major fungal regulator of development and secondary metabolism (Bayram et al., 2008b). VeA activates the sexual life cycle, as $\Delta v e A$ strains do not form any cleistothecia (Kim et al., 2002). VeA-GFP fusion protein abundance was analyzed to investigate if the phenotypical changes in $\triangle u s p A$ are due to a change in the $V e A$ 
protein abundance. Therefore, VeA-GFP fusion protein was expressed under the native promotor of $v e A$ and the abundance of the fusion protein during fungal development was observed in wild type and the $\Delta u s p A^{\text {pyroA }}$ strains. Mycelial samples were taken after $20 \mathrm{~h}$ of vegetative growth and every six hours during subsequent asexual or sexual development. Total fungal crude extracts were prepared and used for western hybridization experiments with a aGFP specific antibody (Figure 39).

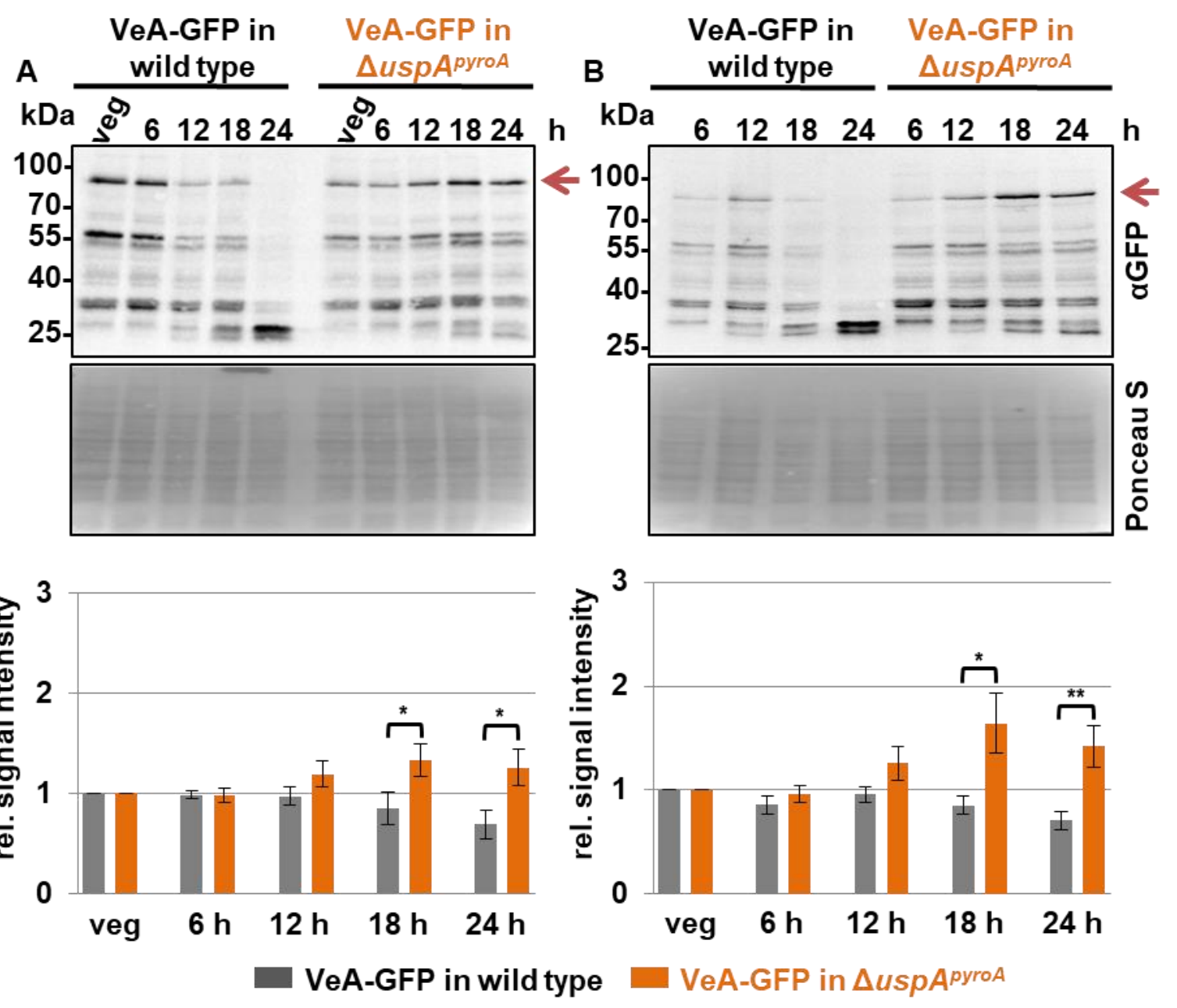

Figure 39: UspA destabilizes VeA-GFP protein abundance during late developmental time points.

Western hybridization of total fungal protein extracts after $20 \mathrm{~h}$ vegetative growth, asexual development (A) or sexual development (B) of VeA-GFP (grey) and VeA-GFP $\Delta u s p A^{\text {pyroA }}$ (orange) strains. Staining with Ponceau $S$ served as loading control. Signal quantification was performed using the BioID software, signals of VeA-GFP fusion protein band (red arrow) were normalized to Ponceau $S$ staining. Quantification results from eight replicates; ${ }^{*} p<0.05$, ${ }^{* *} \mathrm{p}<0.01$. Error bars represent standard error of the mean (SEM). 
The amount of VeA-GFP fusion protein during multicellular development increased in $\Delta u s p A^{\text {pyroA }}$ over time, whereas it decreased in wild type background (Figure 39). A strong signal was observed for VeA-GFP fusion protein in $\Delta u s p A^{\text {pyroA }}$ after $24 \mathrm{~h}$ of asexual or sexual development, whereas hardly any signal was detected in the wild type strain.

VeA seems to be required during vegetative growth and during the initiation of multicellular development of $A$. nidulans, but not anymore during later developmental stages. In the absence of the DUB UspA it accumulates during later stages of development. This indicates that UspA might have an indirect destabilization effect on $\mathrm{VeA}$ or that UspA rather removes single ubiquitin molecules instead of ubiquitin chains, which can target proteins for degradation.

VeA regulates fungal development and secondary metabolism as part of several protein complexes (Sarikaya-Bayram et al., 2015). VeA interacts with the velvet protein VelB and both proteins migrate as a complex into the nucleus (Bayram et al., 2008b). Inside the nucleus they associate with the methyltransferase LaeA to form the trimeric velvet complex, which regulates development and secondary metabolism (Bayram et al., 2008b). The presence of UspA reduces cellular levels of VeA-GFP during multicellular fungal development. Similar western hybridization experiments were performed to investigate if UspA also influences the protein abundance of VelB-GFP (Figure 40).
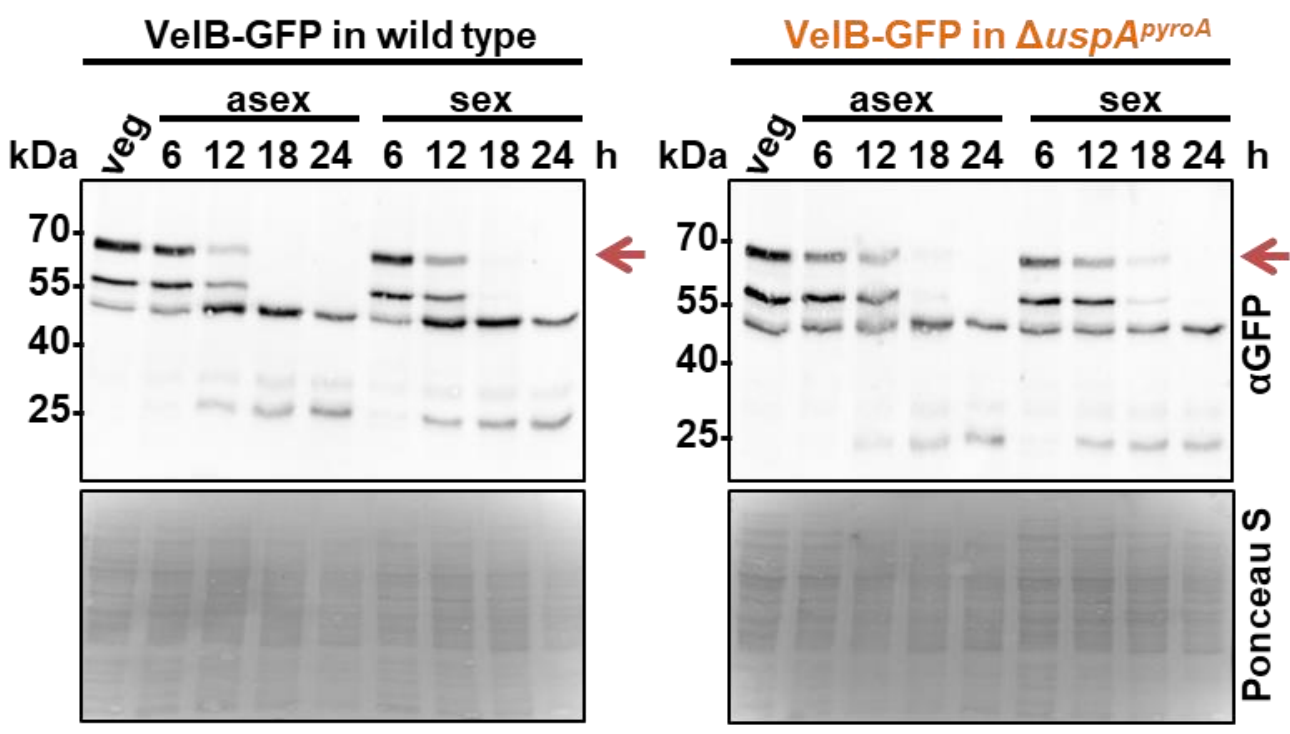

Figure 40: VelB-GFP protein abundance is independent of UspA.

Western hybridization of total fungal protein extracts after $20 \mathrm{~h}$ vegetative growth, asexual development or sexual development of the VelB-GFP (black) and the VelB-GFP $\Delta$ usp $A^{\text {pyroA }}$ (orange) strain. Staining of the nitrocellulose membrane with Ponceau $S$ served as loading control. 
VelB-GFP fusion protein abundance decreases over time during development in pre- and absence of UspA. Comparably high amounts of VelB-GFP are detectable during vegetative growth conditions and during initiation of multicellular development at 6 or $12 \mathrm{~h}$. Protein abundance decreases in both strains after $24 \mathrm{~h}$ of asexual or sexual development (Figure 40).

Taken together, the deubiquitinating enzyme alters VeA-GFP protein abundance during multicellular development, but has no effect on the protein levels of VelB-GFP during fungal development.

\subsubsection{VeA and VelB enter the nucleus and interact with each other independently of the deubiquitinase UspA}

VeA carries a nuclear localization sequence (NLS) in the $\mathrm{N}$-terminal part of the velvet domain (Kim et al., 2002). Interaction with VelB and the nuclear transport factor KapA lead to transfer of the protein complex into the nucleus where it forms the trimeric velvet complex together with the methyltransferase LaeA (Bayram and Braus, 2012; Sarikaya-Bayram et al., 2015). The velvet complex coordinates fungal multicellular development and secondary metabolism (Bayram et al., 2008b). Localization of VeA-GFP and VelB-GFP was investigated in $\Delta u s p A^{p y r o A}$ and wild type strains during vegetative growth conditions (Figure 41).

Strains containing RFP tagged $\mathrm{H} 2 \mathrm{~A}$ were used to visualize nuclei. VeA-GFP expressed in wild type shows a strong nuclear GFP signal (Figure 41A). VeA-GFP in $\triangle u s p A^{\text {pyroA }}$ is also inside the nucleus. (Figure 39). Furthermore, localization of VelB-GFP was investigated in $\triangle u s p A^{\text {pyroA }}$ compared to wild type strain. Similar to VeA-GFP, VelB-GFP accumulates inside the nucleus independently of UspA (Figure 41B).

GFP pull down experiments with subsequent western hybridization and LC/MS-MS analyses were performed to investigate if $\mathrm{VeA}$ is still able to interact with VelB in an uspA deletion strain. Therefore, strains were grown for $20 \mathrm{~h}$ in submerged culture prior to crude extract preparation. A GFP overexpression strain was used as negative control (GFP) and did not pull any of the listed proteins. The bait protein, VeA-GFP, was successfully enriched in both strains (Figure 42A). In LC/MS-MS analyses, VeA was identified with a high number of unique peptides in both background strains indicating that the GFP pull down significantly enriched the bait protein. 
A DIC GFP RFP merge
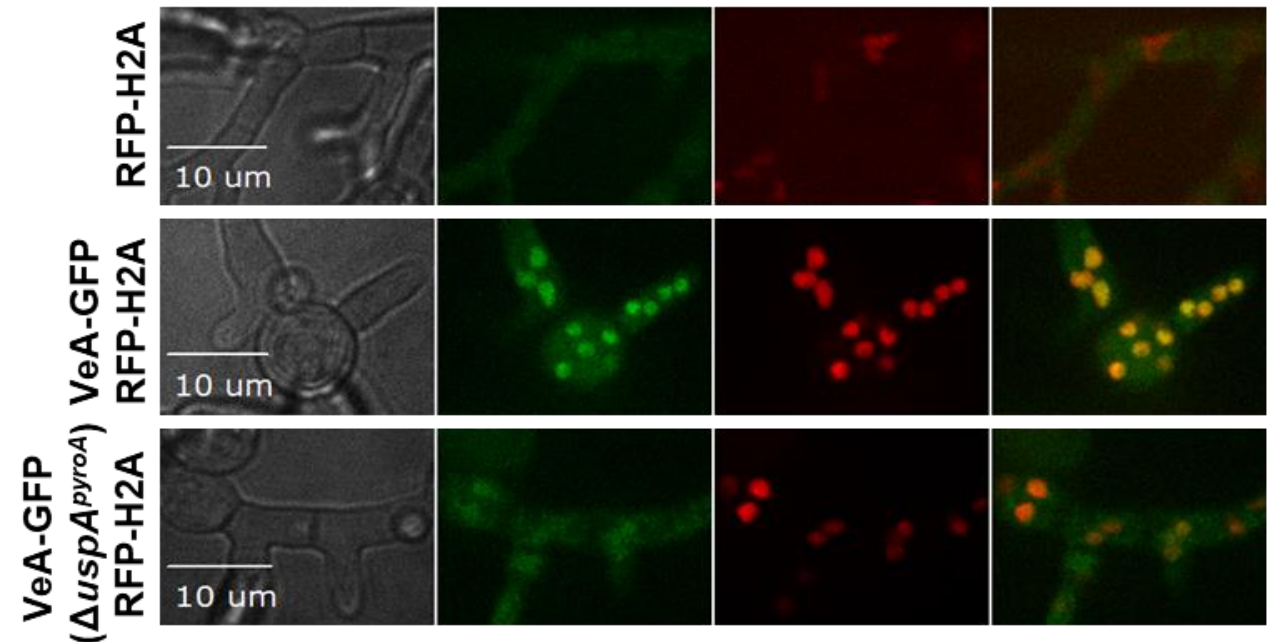

B
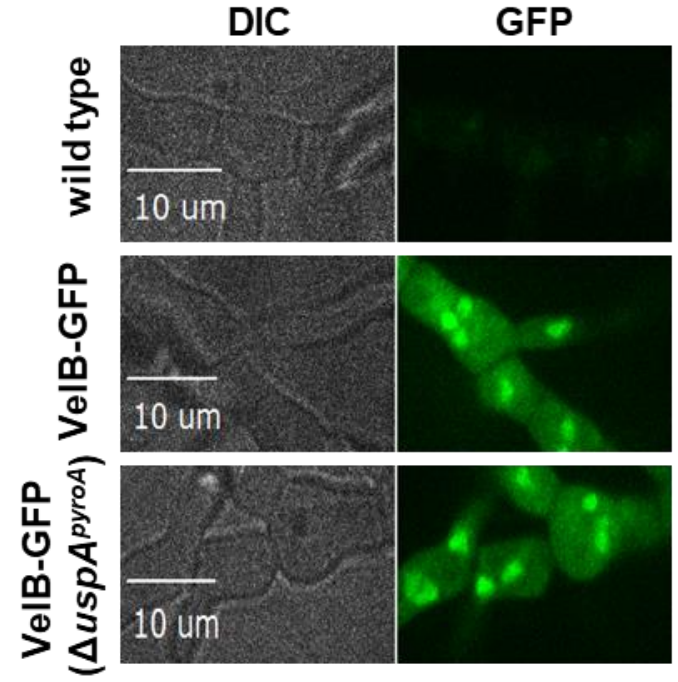

Figure 41: VeA-GFP and VelB-GFP localize inside nuclei independently of the deubiquitinase UspA.

Strains were inoculated with 2,000 spores and grown for $20 \mathrm{~h}$ vegetatively in liquid media on cover slides. A) VeA-GFP localized inside nuclei. H2A was tagged with RFP in all strains to visualize nuclei. In the middle panel VeA-GFP in wild type is depicted, whereas in the lower panel VeA-GFP localization in the $\triangle u s p A^{p y r o A}$ strain is shown. B) In the upper panel a wild type that does not express any GFP fusion protein is depicted. In the middle panel VelB-GFP in wild type and in the lower panel VelB-GFP localization in the $\Delta u s p A^{\text {pyroA }}$ strain is shown. The size bars represent $10 \mu \mathrm{m}$.

VeA-GFP is able to interact with VelB independently of UspA (Figure 42B). VeA-GFP pulls proteins encoded by the secondary metabolite derivative of benzaldehyde (dba) gene cluster, $\mathrm{DbaB}$ and $\mathrm{DbaH}$, both FAD-binding monooxygenases, in $\Delta u s p A^{\text {pyroA }}$ (Figure 42B). The transcript levels of the $d b a$ gene cluster genes in $\Delta u s p A^{p y r o A}$ compared to wild type and complementation 
strains was already investigated (Figure 38). UspA leads to the repression of the genes encoded by the dba gene cluster. Increased transcription levels of dba genes lead to an association of $\mathrm{DbaB}$ and $\mathrm{DbaH}$ with $\mathrm{VeA}$.

Taken together, UspA changes VeA-GFP fusion protein abundance and putative interaction partners, but does not influence the protein levels of VelB-GFP, protein localization or the interaction with VelB and VeA during vegetative growth conditions. VeA associates to proteins encoded by the dba secondary metabolite gene cluster in the absence of the functional deubiquitinase.
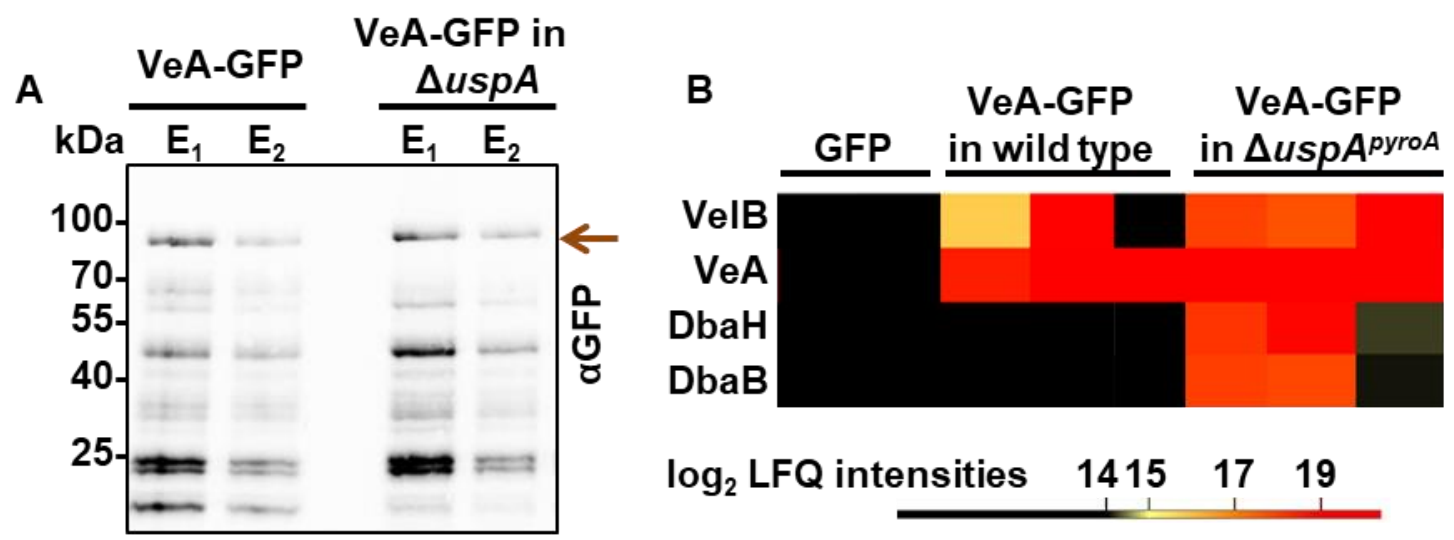

Figure 42: VeA pulls VelB and Dba cluster proteins in $\Delta u s p A^{\text {pyroA }}$ in GFP pull down experiments.

A) Western hybridization of GFP pull down elutions (E) of VeA-GFP in wild type or the $\triangle u s p A^{p y r o A}$ background strain is shown. VeA-GFP signal is highlighted with the red arrow. B) GFP pull downs were in-solution digested and proteins were identified with LC/MS-MS. The data were analyzed with the MaxQuant and Perseus software. The heat map represents $\log _{2}$ LFQ intensities of identified proteins.

UspA belongs to the DUB family of ubiquitin-specific proteases and shares conserved catalytic triad residues and a zinc finger motif with its human ortholog Usp15. It interacts with subunits of the COP9 signalosome in vivo and in vitro. UspA is active throughout fungal development as the pool of ubiquitinated proteins drastically increases in uspA deletion or mutant strains. The ubiquitin-specific protease regulates the protein abundance of the fungal velvet domain containing protein VeA. UspA induces asexual spore formation, is required for early steps of sexual fruiting body formation and causes the repression of genes encoded by the dba secondary metabolite gene cluster. 


\section{Discussion}

The COP9 signalosome interacts physically with E3 ubiquitin ligases, which label target proteins with ubiquitin chains (Cope and Deshaies, 2003; Enchev et al., 2012; Olma et al., 2009). Furthermore, the COP9 signalosome can interact with deubiquitinating enzymes, which remove ubiquitin molecules or chains from proteins (Hetfeld et al., 2005; Zhou et al., 2003). In the present study it was shown that in A. nidulans the balance of ubiquitinated and deubiquitinated proteins is crucial for the formation of asexual conidiospores and the early steps of fruiting body development as well as the concomitant coordinated secondary metabolism. This balance of modified proteins is influenced by the function of the UspA deubiquitinase, which is mainly localized close to nuclei.

\subsection{Regulation of protein abundance by the COP9 signalosome}

The timely coordinated synthesis and degradation of proteins in a cell is essential for accurate development. The COP9 signalosome regulates the activity of cullin RING ligases (CRLs) by removing the ubiquitin-like protein Nedd8 from the cullin subunit (Lyapina et al., 2001). CRLs modify target proteins with ubiquitin (Bosu and Kipreos, 2008; Glickman and Ciechanover, 2002). Polyubiquitinated proteins are substrates for degradation by the $26 \mathrm{~S}$ proteasome (Glickman and Ciechanover, 2002). Hence, the COP9 signalosome is involved in protein half-life control. The embryonal lethality of csn mutants in higher eukaryotes indicates the importance of this protein complex for life (Dohmann et al., 2008; Lykke-Andersen et al., 2003). csn mutants in the reference organism $A$. nidulans are impaired in multicellular developmental programs but are still viable and allow the study of the functions of this protein complex (Beckmann et al., 2015; Busch et al., 2003, 2007). As the COP9 signalosome is involved in protein turnover, the changes of the proteome in strains with a functional COP9 signalosome were compared to a $\triangle c s n E$ deletion strain, which lacks the catalytically active subunit.

In the scope of this study, stable isotope labeling for amino acids in cell culture (SILAC) was established to quantitatively monitor the changes of protein abundances in A. nidulans. To our current knowledge, no $A$. nidulans SILAC study has yet been published. Only one SILAC study, which was performed with Aspergillus spp. so far, used a wild type like A. flavus strain for top-down protein identification and quantification (Collier et al., 2013). Their challenge was to deal with the contamination of endogenous L-arginine, which is synthesized by the fungus itself. 
L-lysine and L-arginine auxotrophic $A$. nidulans strains were generated to enable relative quantification of proteins without contaminations of endogenously synthesized amino acids in the present study. The L-lysine auxotrophic strain constructed in this study was used for the proteome comparison of fungal hyphae containing a defect or an intact COP9 signalosome. The A. nidulans genome encodes for more than 9,000 proteins (Galagan et al., 2005). Less than $10 \%$ (745 proteins) of the fungal proteome were identified during LC/MS-MS measurements. In turn, $13 \%$ of these identified proteins showed differential abundances in A. nidulans strains, which are defective in COP9 signalosome function even though the vegetative growth phenotype is similar in wild type and csn mutant strains.

Most of the proteins decreased in their abundance by CsnE have functions in primary metabolism; they are especially required for amino acid metabolism and vitamin biosynthesis (Table 11). Proteins become labeled with ubiquitin chains by CRLs and subsequently degraded by the $26 \mathrm{~S}$ proteasome (Ciechanover et al., 2000). This leads to a constant supply with amino acids in the fungal cell. In $A$. nidulans strains harboring a csnE deletion, the function of CRLs is disturbed. This leads to changes or a reduction in the amino acid supply and in turn to the upregulation of enzymes that are involved in amino acid metabolism. Vegetative growth is characterized by the formation of a huge hyphal network in a short time, which enables the fungus to colonize different surfaces in a short time (Krijgsheld et al., 2011). For this fast growth, a constant supply of nutrients is essential to synthesize new proteins and vitamins for further growth. A dynamic process of protein biosynthesis and degradation seems to be required for hyphal growth. The COP9 signalosome with its catalytic active subunit CsnE regulates the protein turnover for enzymes that are important for metabolic processes.

The protein abundance of OrsE is increased in $\triangle c s n E$ (Table 11). It is a member of the orsellinic acid secondary metabolite gene cluster and harbors alcohol dehydrogenase activity (Sanchez et al., 2010). The csn mutant strains produce increased amounts of orsellinic acid and its derivatives (Nahlik et al., 2010). Other genes of this gene cluster are upregulated during the disturbed asexual or sexual development in csnE deletion strains (Nahlik et al., 2010). In contrast to the upregulation of protein abundance of OrsE observed in this study, the gene expression was reported to be moderately downregulated after $20 \mathrm{~h}$ vegetative growth in $\Delta c s n E$ (Nahlik et al., 2010). This can have several reasons: First of all, the chosen time point is not exactly the same and differs in 4 hours and also the used $A$. nidulans background strains differ. Secondly, it is not very likely that CsnE influences gene expression directly, but rather protein abundances. Therefore, it is not mutually exclusive that orsE transcripts are downregulated, 
whereas its protein abundance increases in the absence of CsnE during A. nidulans vegetative growth.

The protein abundance of peroxiredoxin, a protein required for the protection against oxidative stress is increased during vegetative, hyphal growth (Rhee et al., 2005). Contratry, the protein abundance of peroxiredoxin was reported before to be decreased in $\Delta c s n E$ mutants (Nahlik et al., 2010). It needs to be taken into consideration that the time point differs slightly and the used method for detecting changes in protein abundances were different in both studies. Anyhow, the differential abundance of proteins related to stress, especially oxidative stress response, is concomitant with the increased oxidative stress susceptibility of csn mutants (Nahlik et al., 2010).

The abundances of several developmental related proteins are increased by CsnE. Four different septins, AspA, $-B,-C$ and $-D$ were identified to be decreased in their abundance in $\triangle c s n E$ (Table 12). A. nidulans carries five different septin proteins (Hernández-Rodríguez et al., 2012; Momany et al., 2001). Septins are required for accurate hyphal growth and asexual development. Thereby, AspA-D are proposed to work together in a complex to control development, whereas AspE acts probably as single protein in a more specialized pathway (Momany et al., 2001). This is corroborated here by the fact that AspE is the only septin, which is not altered in its protein abundance by the lack of CsnE. Deletions of aspA or aspC genes lead to a formation of several germ tubes, early branching and altered septae positioning (Lindsey et al., 2010). The displacement of septae in csn deletion strains of $A$. nidulans and concomitant formation of short cells was observed previously (Busch et al., 2003). This might be ascribed to the decreased abundance of Septin proteins in a $\Delta c s n E$ strain. Furthermore, a number of other proteins predicted to be involved in the regulation of hyphal growth are positively influenced by CsnE. One of them is the glucose-methanol-choline (GMC) oxidoreductase A (Table 12). GmcA is essential for the induction of conidiophore formation (Etxebeste et al., 2012). Several other GTP binding proteins with a predicted role in cytoskeleton formation showed different protein abundances in $\Delta c s n E$.

Taken together, this shows that the SILAC approach works in $A$. nidulans to quantify the abundances of proteins deriving from different cultures. Furthermore, the observed csn deletion phenotypes can be assigned to differentially regulated proteins in the absence of a functional COP9 signalosome. Due to the high conservation of the ubiquitin-proteasome system from fungi to mammals, new insights into CRL targets influenced by the COP9 signalome under different growth conditions or stress factors can be gained in future studies by applying this approach. 
It needs to be taken into consideration that the relative quantification of protein abundances with SILAC in the scope of this study does not reflect a complete pattern of fungal proteins. The use of L-lysine as only isotopically-labeled amino acid leads to the fact that only L-lysine containing peptides can be used for quantification of protein abundances. The sample preparation for the required LC/MS-MS analyses was performed with the endopeptidase trypsin. It cleaves proteins after L-lysine or L-arginine residues into peptides (Simpson, 2006). According to the amino acid incorporation test, $60 \%$ of all identified peptides contain L-lysine residues. This means that $40 \%$ of the peptides cannot be used for relative quantification of protein abundances. A similar experiment using the $\mathrm{L}$-arginine auxotrophic $A$. nidulans strain is required to get a more complete spectrum of regulated proteins in $\triangle c s n E$. Furthermore, a label-swap experiment needs to be conducted to exclude the possibility that the detected differential protein abundances are artefacts of the labeling procedure. Furthermore, the SILAC approach should be applied during A. nidulans multicellular development. Quantitative changes in protein abundances during asexual and sexual development will reveal new insights into proteins that are regulated by CsnE. Analysis of the fungal transcriptome in $\Delta c s n E$ compared to wild type strains during multicellular fungal development revealed a long list of regulated candidates related to fungal development and secondary metabolism (Nahlik et al., 2010). However, as subunit of the COP9 signalosome CsnE is rather likely to affect protein abundances instead of gene transcription. SILAC in $A$. nidulans provides an appropriate tool to track these differences also on the proteome level.

\subsection{Aspergillus nidulans has a diverse repertoire of deubiquitinating enzymes}

The ubiquitination signal is one of the most diverse posttranslational modifications known so far. The complexity is conferred by different ubiquitin chain linkage types and posttranslational modifications of the ubiquitin chains itself (Ikeda and Dikic, 2008; Komander et al., 2009). Besides the well-known linkages through lysine residues and the initial methionine residue, attachment of ubiquitin on non-lysine residues like cysteines was observed (Cadwell et al., 2005). In higher eukaryotes at least ten different ubiquitin-like proteins such as Nedd8, Sumo, interferon stimulated gene 15 (ISG15), or the autophagy-related protein 8 (Atg8) exist, which all share a common three-dimensional $\beta$-grasp fold, but show only low primary sequence similarities (Kerscher et al., 2006). The ubiquitin-like proteins Sumo, Nedd8 or ISG15 can be incorporated into ubiquitin chains (Fan et al., 2015; Ikeda and Dikic, 2008). Apart from ubiquitin, 
other UBL proteins like Sumo or Nedd8 are able to build chains that modify proteins as well (Bylebyl et al., 2003; Jones et al., 2009; Ohki et al., 2009; Ulrich, 2008).

The attachment of UBL proteins is reversible. Six different proteins are capable of removing the UBL protein Sumo from target proteins in mammals (Mukhopadhyay and Dasso, 2007; Wang and Dasso, 2009). Two desumoylation proteins were described for $S$. cerevisiae (Li and Hochstrasser, 2000; Wang and Dasso, 2009). The COP9 signalosome and the deneddylase protein 1 (DEN1) are conserved from fungi to humans and enable the removal of Nedd8 modifications (Cope et al., 2002; Gan-Erdene et al., 2003). The preferred targets of COP9 signalosome deneddylation are the cullin proteins, which in turn are responsible for ubiquitination reactions (Wei and Deng, 2003). The Den1 protein in Arabidopsis thaliana or D. melanogaster deneddylates preferably non-cullin proteins (Chan et al., 2008; Mergner et al., 2015). In A. nidulans, DenA is involved in the asexual development, whereas the COP9 signalosome is essential for completion of the sexual life cycle and for an appropriate light response (Christmann et al., 2013). This also indicates different targets for deneddylation of the two proteins in A. nidulans (Christmann et al., 2013).

Deubiquitinating enzymes (DUBs) reverse the function of the UPS by removing ubiquitin molecules or chains from target proteins. Six families of deubiquitinating enzymes exist in

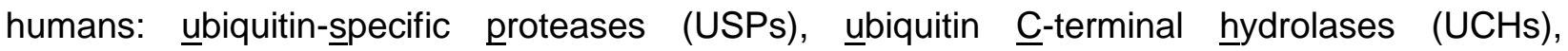
ovarian-tumor proteases (OTUs), Machado-Josephin domain proteases (MJDs), JAMM containing DUBs and the recently characterized motif interacting with Ub-containing novel DU family (MINDY) (Komander et al., 2009; Abdul Rehman et al., 2016). The group with the by far most members is the ubiquitin-specific protease family (Hutchins et al., 2013). The metalloprotease JAMM domain and OTU domain proteases follow with approximately one fourth as much proteins as the USP family. The UCH and MJD family comprise only four members in human, respectively. Humans harbor more than 80 DUB proteins in total (Hutchins et al., 2013; Komander et al., 2009; Nijman et al., 2005). D. melanogaster has less DUBs than humans, but they are distributed in similar ratios to the different subfamilies (Tsou et al., 2012). S. cerevisiae expresses 22 DUBs with two-thirds belonging to the USP family. MJD domain proteases are absent in the yeast genome (Amerik and Hochstrasser, 2004; Hutchins et al., 2013).

An in silico analyses of gene loci assigned to deubiquitinating activity in FungiDB and AspGD revealed 22 different genes encoding proteins with putative deubiquitination activity in A. nidulans (Table 17). BLAST analyses of the amino acid sequences of these different putative $A$. nidulans DUBs against the $H$. sapiens database (taxid: 9606) allowed the classification of the different fungal DUBs to the different subfamilies (Table 17, Figure 43). 
Table 17: An overview about deubiquitinating enzymes in $A$. nidulans.

In silico analyses with FungiDB and AspGD revealed 22 putative deubiquitinating enzymes (Cerqueira et al., 2014; Stajich et al., 2012). BLAST analyses against the $H$. sapiens database (taxid: 9606) allowed the classification of the proteins to the different DUB families (Altschul et al., 1990).

\begin{tabular}{|c|c|}
\hline Systematic name & Description \\
\hline & Ovarian tumor proteases (OTU) \\
\hline AN5638 & Orthologs have ubiquitin-specific protease activity \\
\hline AN3449 & Orthologs have ubiquitin-specific protease activity \\
\hline \multicolumn{2}{|c|}{ JAB1/MPN/ Mov34 metalloenzyme domain containing DUBs (JAMM) } \\
\hline AN4492 & Orthologs have ubiquitin-specific protease activity \\
\hline AN3003 & Orthologs have ubiquitin-specific protease activity \\
\hline \multicolumn{2}{|r|}{ Ubbiquitin $\underline{\text { C-terminal hydrolases (UCH) }}$} \\
\hline AN7491 & Putative C-terminal hydrolase \\
\hline AN0927 & Orthologs have ubiquitin-specific protease activity \\
\hline AN3453 & Has domains with predicted ubiquitin-specific protease activity \\
\hline AN11218 & Has domains with predicted ubiquitin-specific protease activity \\
\hline \multicolumn{2}{|r|}{ Ubiquitin-specific proteases (USP) } \\
\hline AN6354 & Ubiquitin-specific protease A, UspA, analyzed in this study \\
\hline AN2072 & Putative ubiquitin-specific protease \\
\hline AN3711 & Orthologs have histone deubiquitination activity \\
\hline AN3587 & CreB, ubiquitin processing protease \\
\hline AN5186 & Orthologs have ubiquitin-specific protease activity \\
\hline AN7422 & Putative ubiquitin carboxyl-terminal hydrolase \\
\hline AN6913 & Orthologs have ubiquitin-specific protease activity \\
\hline AN2507 & Has domains with ubiquitinyl hydrolase activity \\
\hline AN11102 & Orthologs have ubiquitin-specific protease activity \\
\hline AN10722 & Orthologs have ubiquitin-specific protease activity \\
\hline AN11684 & Orthologs have ubiquitin-specific protease activity \\
\hline AN6164 & Has domains with ubiquitinyl hydrolase activity \\
\hline AN8074 & Has domains with ubiquitinyl hydrolase activity \\
\hline \multicolumn{2}{|c|}{ Motif interacting with Ub-containing novel DUU family (MINDY) } \\
\hline AN8067 & Thiol dependent ubiquitin-specific protease activity \\
\hline
\end{tabular}

The two A. nidulans proteins AN5638 and AN3440 were classified as members of the OTU domain protein family. Furthermore, two proteins could be assigned to the JAMM domain metalloproteases including AN4492, which is the catalytic active subunit of the proteasomal LID. 
Four proteins show similarities to members of the UCH family, namely AN7491, AN0927, AN3453 and AN11218. Like for humans, D. melanogaster and S. cerevisiae, the USP family forms the largest group with 13 members in A. nidulans (Table 17). One protein, AN8067, was assigned to the recently identified MINDY DUB family. No $A$. nidulans protein with putative deubiquitinating activity could be classified to the MJD domain containing proteases.

The number of the different DUB family members in $H$. sapiens, $D$. melanogaster, $S$. cerevisiae and $A$. nidulans are depicted in Figure 43. OTUs have 16 members in humans and 14 in D. melanogaster (Mevissen et al., 2013; Tsou et al., 2012). Members of this DUB family often show specificity for a certain ubiquitin linkage type. Furthermore, catalytic activity towards ISG15, but not against Nedd8 was observed (Frias-Staheli et al., 2007; Mevissen et al., 2013). In silico approaches identified two genes encoding putative OTU family members in yeast (Hutchins et al., 2013). Two A. nidulans proteins could be assigned to this family due to BLAST analyses performed during this study.

Four members of MJD proteases are known in humans and five in D. melanogaster (Eletr and Wilkinson, 2014; Hutchins et al., 2013; Tsou et al., 2012). Ataxin-3, the best-studied member of the MJD family, binds long K63 or K48 ubiquitin chains (Eletr and Wilkinson, 2014; Winborn et al., 2008). No orthologs have been found in S. cerevisiae (Hutchins et al., 2013). To the best of our current knowledge also in A. nidulans no MJD family member has been identified. In silico analyses of the genome of Arabidopsis thaliana revealed three putative members of the MJD class (Isono and Nagel, 2014). Furthermore, at least one representative of the MJD class exists in Caenorhabditis elegans (Rodrigues et al., 2007). According to KEGG database searches for MJD family members, basidiomycetes like Ustilago maydis and Puccinia graminis contain one hypothetical MJD protein, whereas other ascomycetes like A.fumigatus, $A$. niger, Sordaria macrospora, Neurospora crassa or Verticillium dahliae do not have any representative of this subfamily (Kanehisa et al., 2017). The MJD family might have been lost in the phylum of ascomycetes. Nevertheless, the presence of a yet unknown Machado-Josephin domain containing protein in $A$. nidulans or other ascomycetes cannot be completely ruled out.

Twelve genes encoding metalloprotease DUBs were identified in human (Hutchins et al., 2013; Nijman et al., 2005) and eleven in D. melanogaster (Tsou et al., 2012). The most prominent member is the catalytically active subunit of the proteasomal LID Rpn11, which deubiquitinates substrates prior to their degradation by the $26 \mathrm{~S}$ proteasome. Two putative metalloprotease DUBs were identified during in silico analyses in $A$. nidulans including AN4492, which is supposed to be the ortholog of the internal LID deubiquitinase. 
Four members of the recently discovered MINDY family were identified in humans and two in S. cerevisiae (Abdul Rehman et al., 2016). Bioinformatical studies revealed three representatives in A. thaliana and one in D. melanogaster (Abdul Rehman et al., 2016). Members of the MINDY family show high specificity to K48-linked ubiquitin chains (Abdul Rehman et al., 2016). In the scope of BLAST analyses performed in the present study of human MINDY proteins against the $A$. nidulans database, one putative MINDY family protein was identified in A. nidulans, which is AN8067.

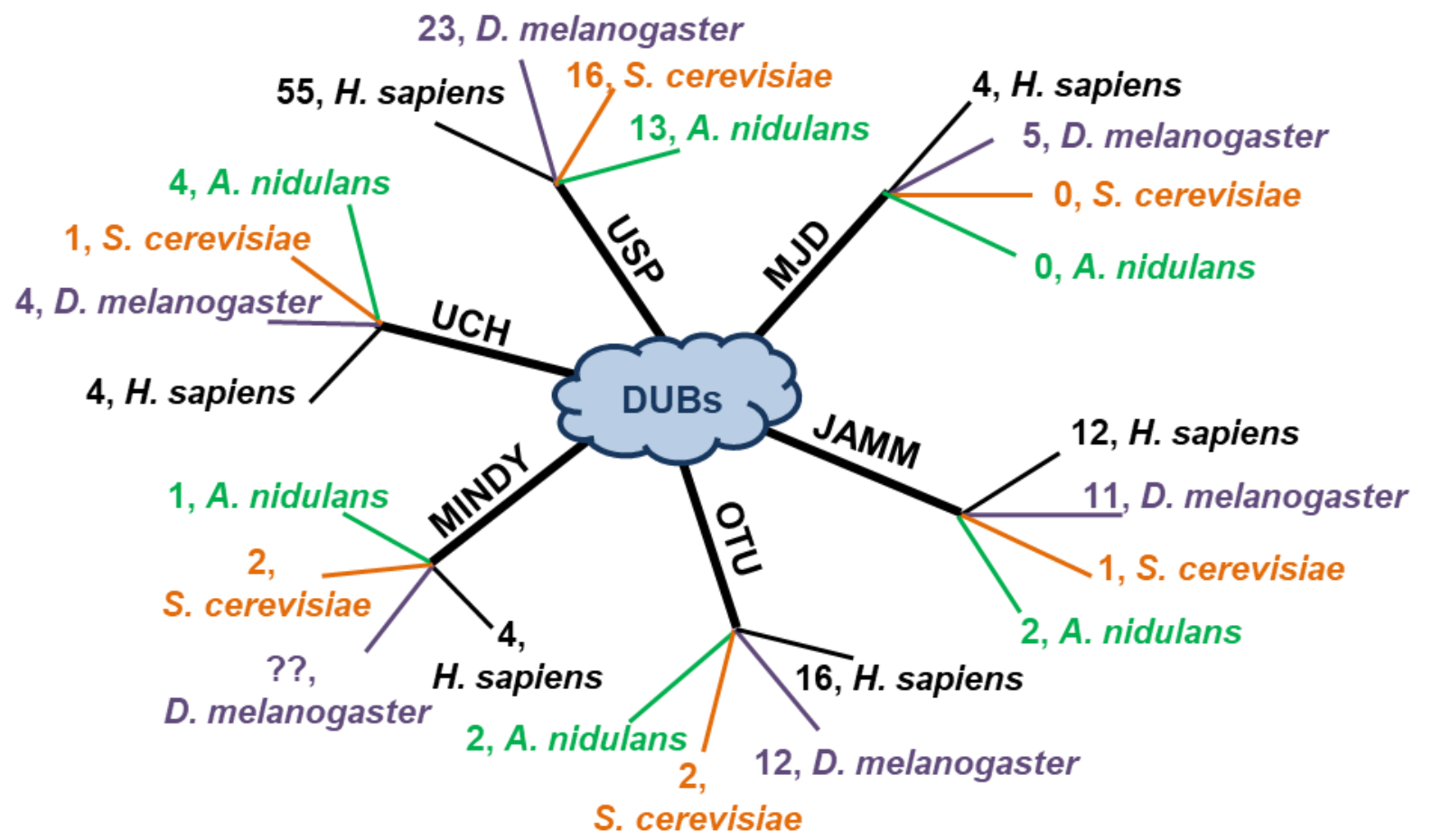

Figure 43: Deubiquitinating enzymes can be divided into six subfamilies.

Schematic representation of members of the different DUB families in $H$. sapiens, $D$. melanogaster, S. cerevisiae and A. nidulans are depicted. Numbers of D. melanogaster DUBs rely on an analysis performed by Tsou and co-workers (Tsou et al., 2012). The assignment of human and yeast DUBs to the single subfamilies is based on different published studies and entries in the KEGG database (Amerik et al., 2000; Hutchins et al., 2013; Nijman et al., 2005; Abdul Rehman et al., 2016). The classification of $A$. nidulans DUBs was performed with BLAST and text-based database searches in the scope of this study.

The DUB family of UCHs encompasses four members in human and in D. melanogaster, respectively (Hutchins et al., 2013). S. cerevisiae only contains one UCH protein, called YUH1, which gene deletion does not have deleterious effects for yeast viability (Miller et al., 1989). This suggests overlapping function with DUBs belonging to other families (Johnston et al., 1999). 
$\mathrm{UCH}$ family proteins are tissue specific and for this reason their functions are diverse, ranging from histone modification and chromatin remodeling to proteasome-associated deubiquitination activity in higher eukaryotes (Bishop et al., 2016; Yao et al., 2006; Yu et al., 2010). Human UCH-L1 and UCH-L3 have also high affinities to the UBL protein Nedd8, whereas only UCH-L3 is able to catalyze hydrolysis reactions (Bishop et al., 2016; Wada et al., 1998). Database searches and BLAST analyses revealed four $A$. nidulans proteins that belong to the UCH DUB family.

The USPs constitute the largest family of DUBs with 55 members in humans and 23 in D. melanogaster (Hutchins et al., 2013; Tsou et al., 2012). 16 USP proteins are described in S. cerevisiae (Amerik et al., 2000). The deletion of all single USP encoding genes in yeast revealed that none of them is essential for fungal life. The loss of one ubiquitin-specific protease does not lead to severe growth defects, most probably due to overlapping deubiquitinating function (Amerik et al., 2000). Bioinformatic analyses of the DUB encoding genes in the parasitic worm Schistosoma mansoni revealed 17 USP members, which are differentially regulated through the whole life cycle (Pereira et al., 2015). Therewith, USPs regulate a broad range of substrates during the parasitic life cycle (Pereira et al., 2015). Thirteen out of 22 different proteins in A. nidulans that have putative deubiquitinase activity were assigned to the USP subfamily. Only one of them, CreB, is characterized so far (Adnan et al., 2018; Lockington and Kelly, 2001, 2002). CreB is involved in carbon catabolite repression and loss of function mutants do not influence viability on complete medium, but result in growth defects on different synthetic media (Lockington and Kelly, 2001). Ubiquitin-specific proteases show rarely specificity for a certain linkage type, but are rather able to recognize a broad range of polyubiquitin chains (Hanpude et al., 2015; Komander et al., 2009). The substrates of USPs are very diverse, which leads to an impact of USPs in many cellular signaling pathways, DNA damage or stress response and their dysfunction cause several human diseases (Clague et al., 2012; Hicke and Dunn, 2003; Huang et al., 2009; Li et al., 2002; Mukai et al., 2010; Nicassio et al., 2007). USPs have long C-terminal extensions, which are involved in protein-protein interactions and determine the subcellular localization of the enzymes (Reyes-Turcu et al., 2009).

Human Usp15, Usp11 and Usp4 are paralogous DUBs. All proteins share common domain architectures, whereas Usp15 and Usp4 share the highest similarities in their amino acid sequence (Harper et al., 2011; Vlasschaert et al., 2015). They share an N-terminal DUSP domain, which is followed by an UBL domain. These domains are connected through a DU finger (Harper et al., 2011). The DUSP domain surface suggests a role in protein-protein interaction or substrate recognition (de Jong et al., 2006). All enzymes share a long USP 
domain, which encompasses a second UBL domain. This USP domain contains the conserved cysteine, histidine and aspartate residues that built the catalytic triad (Faronato et al., 2011). The three human paralogous proteins are represented in $S$. mansoni in one enzyme called Smp_128770 and it contains the conserved N-terminal DUSP domain (Pereira et al., 2015). BLAST analyses of Usp15 (Uniprot ID: Q9Y4E8), Usp11 (Uniprot ID: P51784) and Usp4 (Uniprot ID: Q13107) amino acid sequences derived from Uniprot against the $S$. cerevisiae or S. pombe database reveals as best hit always ScUBP12 or SpUbp12, respectively. Consistently, BLAST analyses against the database of $A$. nidulans reveals as best hit always AN6354/UspA. This indicates that the three paralogous proteins in mammals had a common ancestor and are represented in the fungal kingdom as one USP protein.

\subsection{DUBs are often incorporated into multiprotein complexes during deubiquitination reactions}

Numerous studies on different DUBs indicated that a majority of human DUBs is associated to multiprotein complexes (Sowa et al., 2009). The catalytic activity of some DUBs is dependent on the incorporation into large protein complexes, but the interaction might also lead to the regulation of subunits of the complex itself through deubiquitination reactions (Sowa et al., 2009).

The UCH protein Bap1 forms stable complexes with the host cell factor 1 (HCF-1), which is a transcriptional regulator (Yu et al., 2010). Bap1 deubiquitinates HCF-1 and the Bap1-HCF-1 complex influences interactions of the deubiquitinase with other putative target proteins and therewith regulates its targeted enzymatic activity (Machida et al., 2009; Yu et al., 2010).

The yeast DUB Ubp8 was identified as component of the Spt/Ada/Gen5L acetyltransferase (SAGA) complex, which is a transcriptional cofactor complex, during global protein interaction analysis and has deubiquitination activity towards histones (Henry et al., 2003; Kouranti et al., 2010). Usp22 was identified as a new subunit of the human SAGA complex and is able to deubiquitinate histones as well (Zhang et al., 2008).

Human Usp3 and Usp39 interact with subunits of the eukaryotic initiation factor 3 (elF3) complex and were identified to interact with phosphatase scaffolding or mRNA processing complexes (Sowa et al., 2009).

Three different DUBs are associated to the 26 proteasome, namely Usp14, UCH37 and Rpn11 (de Poot et al., 2017; Sowa et al., 2009; Yao et al., 2006). The metalloprotease DUB Rpn11 is 
the catalytically active isopeptidase subunit of the proteasomal LID. It exerts significantly reduced deubiquitinating activity when it is not incorporated into the LID complex (Pathare et al., 2014). Similar regulation of enzyme activity due to conformational changes induced by complex formation was observed in Csn5, the catalytic active subunit of the COP9 signalosome (Lingaraju et al., 2014; Meister et al., 2016). Human Usp15 and its ortholog in S. pombe interact with the COP9 signalosome (Hetfeld et al., 2005; Schweitzer et al., 2007; Zhou et al., 2003). The COP9 signalosome is responsible to ensure the stability of the CRL complex and especially its substrate adaptor/receptor complexes due to its association with deubiquitinases (Wee et al., 2005). It was shown that the fission yeast ortholog Ubp12, which interacts with the COP9 signalosome, represses the catalytic activity of the E3 ligase Pcu3p and stabilizes the substrate adaptor Pop1p (Zhou et al., 2003). Similarly, Usp15 protects the substrate adaptor Rbx1 from autoubiquitination (Hetfeld et al., 2005). In this study it was shown that A. nidulans UspA interacts with subunits of the COP9 signalosome in vivo and in vitro.

\subsubsection{CsnE is required for regulation of the expression of DUB encoding genes}

Deubiquitinating enzymes reverse the ubiquitination process catalyzed by E3 ubiquitin ligases. The activity of E3 ligases, like SCF complexes, is influenced by the COP9 signalosome (Lyapina et al., 2001; Wei and Deng, 2003). This eight-subunit protein complex binds SCF complexes, which do not carry a substrate, remove the Nedd8 molecule from the cullin subunit and lead to the exchange of adaptor/receptor complexes. The posttranslational modification of cullins with Nedd8 support CRL activity (Hotton and Callis, 2008; Morimoto et al., 2000; Wu et al., 2002). The Nedd8 modification is required for binding of E2 enzymes to the E3 ligases, which deliver the activated ubiquitin molecules to the CRL E3 ligase (Hotton and Callis, 2008; Kawakami et al., 2001). Therefore, the COP9 signalosome ensures the targeted ubiquitination of substrates and is involved in the protein half-life control of many proteins. The subunit CsnE of the COP9 signalosome confers the catalytic deneddylation activity to the complex (Cope et al., 2002). Cullins are constantly neddylated in $\triangle c s n E$ strains (Beckmann et al., 2015). The constant neddylation of CRLs in $\triangle c s n E$ might lead to increased or unspecific ubiquitination of substrates or to autoubiquitination of CRL complex components (Cope and Deshaies, 2006).

A. nidulans possibly counteracts this process with the upregulation of genes encoding for ubiquitin-specific proteases during multicellular fungal development (Figure 21, Figure 31). Even though the expression of all tested USP encoding genes was upregulated, the highest increase was observed for uspA. CsnE does probably not directly affect the transcription of the USP 
encoding genes. A likely scenario would be that the COP9 signalosome regulates the stability of a yet unknown transcriptional activator or repressor, which in turn regulates the expression levels of the usp encoding genes (Figure 44). Furthermore, the ubiquitination of the transcriptional regulator could also change its localization and therewith lead to the regulation of usp gene transcription.

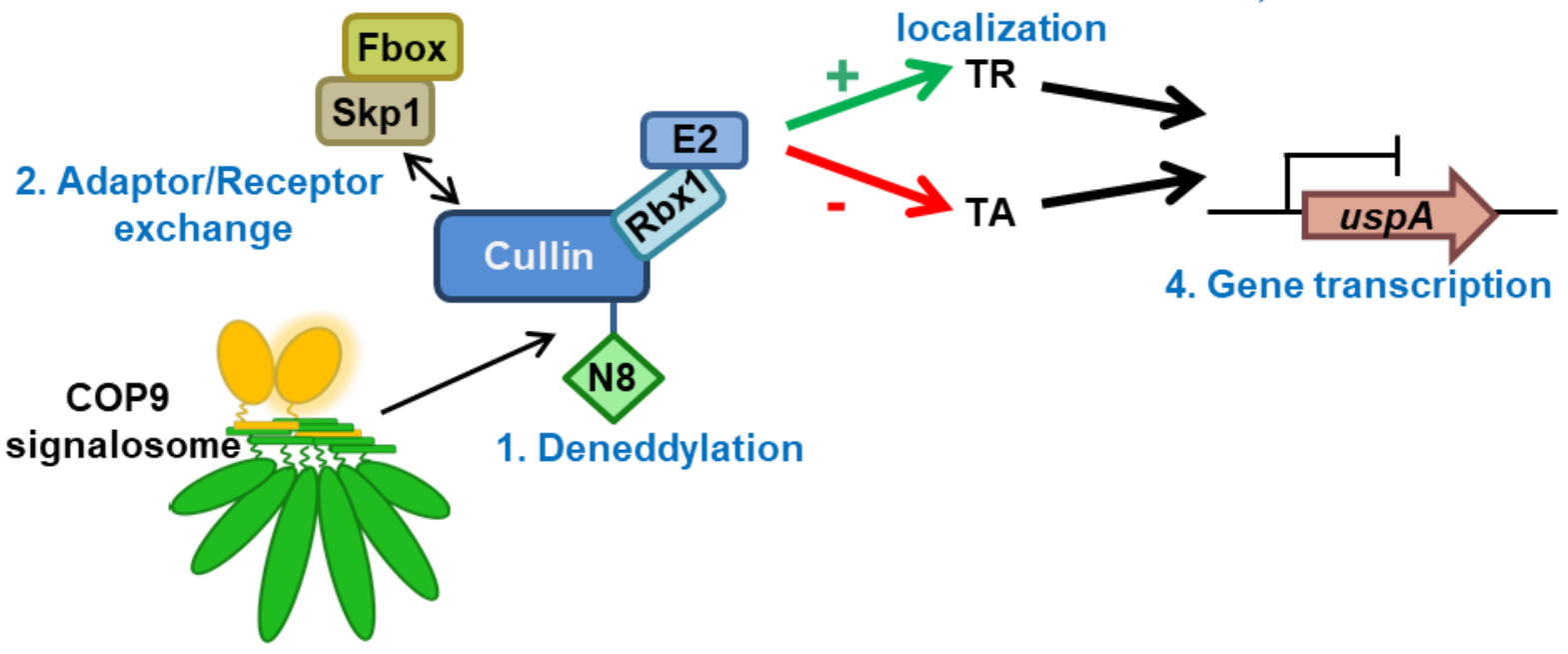

\section{Substrate}

stabilization/ destabilization, localization

Figure 44: The COP9 signalosome influences uspA gene transcription.

The COP9 signalosome deneddylates CRLs that are not associated to substrates. Removal of Nedd8 (N8) catalyzed by the catalytic active subunit CsnE enables adaptor/receptor exchange of CRLs. This ensures targeted ubiquitination of substrates. Thereby, the stability of possible transcriptional activators (TA) or transcriptional repressors (TR) might be changed, which in turn change the gene expression pattern of the ubiquitin-specific protease encoding gene uspA.

The upregulation of transcription levels of $u s p$ encoding genes was observed during $A$. nidulans multicellular development and not during the state of developmental competence. The transcript and protein levels of the other DUB family members in the absence of CsnE still remains to be tested.

A functional CSN complex in fungal cells inhibits the accumulation of CRLs that are bound to the substrate adaptors Fbox15 or Fbox23 (von Zeska Kress et al., 2012). In $\Delta c s n E$ strains these complexes accumulate. Both Fbox proteins are important for the regulation of fungal development (von Zeska Kress et al., 2012). It is possible that UspA influences the stability of these Fbox proteins or its substrates during complex formation with the COP9 signalosome or as single protein. 


\subsubsection{UspA interacts with subunits of the COP9 signalosome}

Protein BLAST analyses revealed UspA encoded by AN6354 as Usp15 ortholog in A. nidulans. Yeast-two hybrid interaction studies were performed in the scope of this study and suggest an interaction of UspA with six of the eight CSN subunits (Figure 20). UspA shows positive interaction signals with the two MPN-domain containing subunits CsnE and CsnF and four of the $\mathrm{PCl}$ domain containing subunits. No interaction was detected with $\mathrm{PCl}$ domain containing proteins CsnC and CsnG. The crystal structure of human COP9 signalosome revealed that all $\mathrm{PCl}$ domain containing proteins form a ring like structure, whereas the two MPN domain containing subunits are sitting on top of this ring (Lingaraju et al., 2014). In this ring, Csn3 (the corresponding subunit to $A$. nidulans $\mathrm{CsnC}$ ) and Csn7 (CsnG) are far apart from each other. All subunits are connected through the $\mathrm{C}$-terminal $\alpha$-helices, which form a helical bundle (Figure 45).

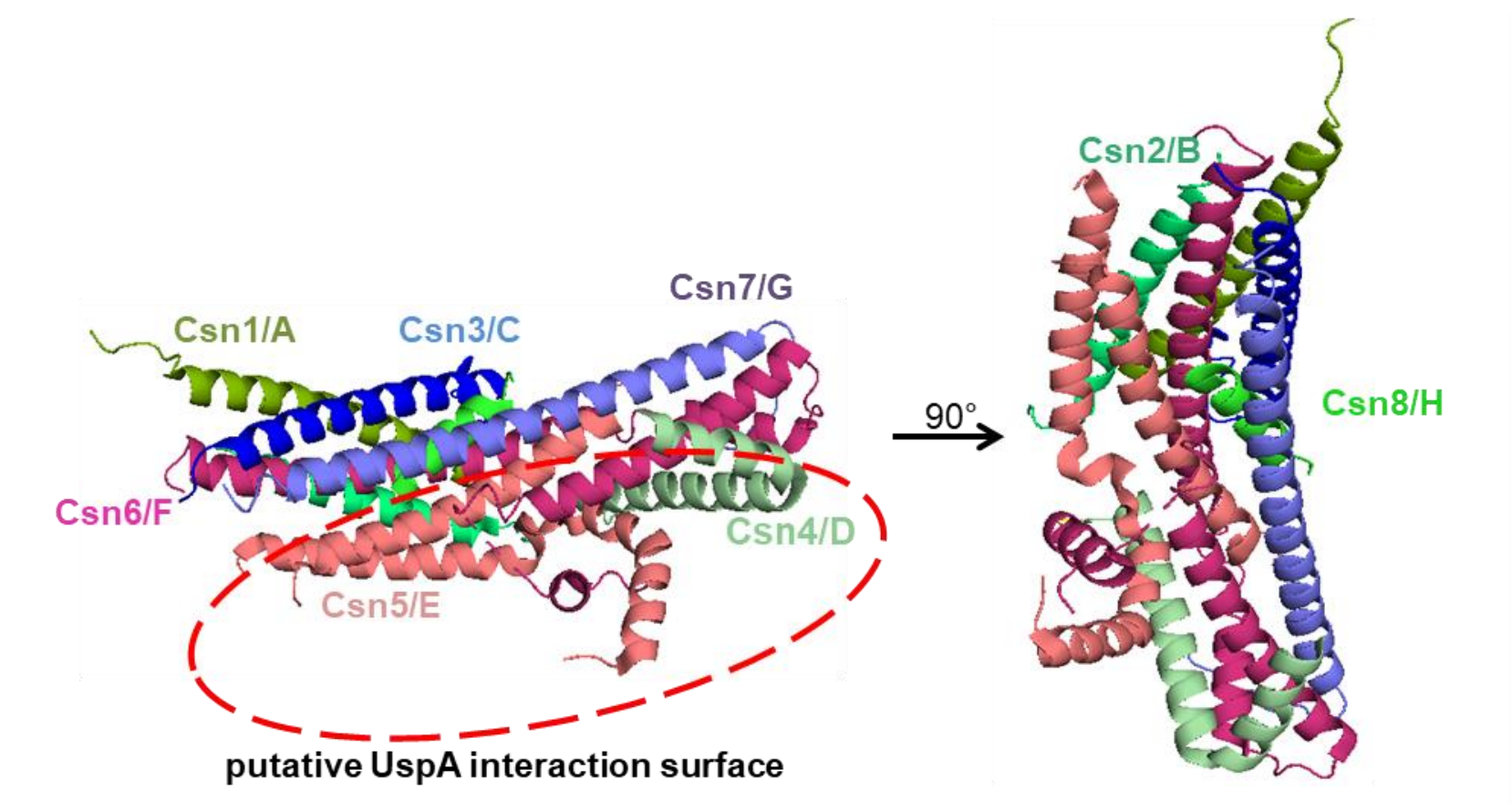

Figure 45: Cartoon representation of the human COP9 signalosome helical bundle.

Only the $\alpha$-helices building the C-terminal helical bundle of the single CSN subunits are shown. The human crystal structure of human COP9 signalosome (PDB 4d10) served as basis and was edited with the PyMol 2.0 Software (Lingaraju et al., 2014). Csn3 and Csn7 are depicted in shades of blue, MPN domain containing subunits are depicted in shades of pink and all other subunits in green. Csn3 and Csn7 are located at one side of the helical bundle, whereas all others protrude to the other side. The red circle depicts a possible binding surface of UspA with the helical bundle. 
In this helical bundle, helices of Csn3 and Csn7 are protruding in a different direction than all others. The other C-terminal helices provide a putative UspA interaction surface. Therefore, UspA might bind to the helical bundle of the COP9 signalosome.

Ubp12, the orthologous protein of human Usp15 in S.pombe, was identified during the isolation of the COP9 signalosome (Zhou et al., 2003). This DUB conferred the observed deubiquitination activity to the COP9 signalosome. Direct co-purifications of Usp12 with Csn1, Cns3, Csn5 and Csn7b could be shown (Zhou et al., 2003). UspA does not interact directly with CsnC or CsnG in vitro. An indirect interaction of UspA with CsnC or CsnG in vivo might be possible through the interaction with other CSN subunits. The co-purification of the single COP9 signalosome subunits in $S$. pombe with Ubp12 does not necessarily indicate a direct interaction between these proteins. If Ubp12 interacts only with some of the CSN subunits, the other components are also co-purified as they interact with the respective Ubp12p interacting subunit. Similar copurification experiments revealed the interaction of human COP9 signalosome with Usp15, but the interaction was not mapped to a specific CSN subunit (Hetfeld et al., 2005).

Bimolecular fluorescence complementation microscopy experiments confirmed the direct interaction between UspA and CSN subunits in vivo in A. nidulans (Figure 24). CsnB and CsnF were chosen as in vitro experiments suggested the strongest interaction between both proteins and UspA, respectively. Sharon and co-workers suggested due to native mass spectrometry experiments the formation of two CSN modules: one consisting of Csn1, 2, 3 and 8 and the other one consisting of Csn4, 5, 6 and 7 (Sharon et al., 2009). The interaction with CsnB and CsnF indicates that UspA associates to both modules and therefore most likely with the full COP9 signalosome.

GFP pull down experiments with an active and an inactive UspA-GFP fusion protein during A. nidulans vegetative growth conditions did not co-purify any CSN subunits. The interaction of UspA and CSN might be either only transiently or might only take place under specific conditions. Human Usp15 interacts with the COP9 signalosome to stabilize IkBa after TNF treatment (Schweitzer et al., 2007). The interaction of the COP9 signalosome with UspA might be restricted to certain developmental stages or stress conditions. GFP pull downs under these conditions might lead to co-purification of CSN subunits.

\subsubsection{CsnE protein levels are independent of UspA}

Proteins labeled with K48 linked polyubiquitin chains are sentenced for degradation to the $26 \mathrm{~S}$ proteasome (Finley et al., 1994; Glickman and Ciechanover, 2002). Some DUBs have the ability 
to protect proteins for degradation by removal of the ubiquitin chain and their target proteins are less stable in their absence (Hartmann-Petersen et al., 2003; Komander et al., 2009). Alternatively, removal of ubiquitin molecules or chains connected through other lysine residues than K48 can have regulatory function on proteins (Spasser and Brik, 2012).

In the scope of this study it was investigated if the catalytically active subunit CsnE from the COP9 signalosome is a target for deubiquitination by UspA. The CsnE protein abundance was not influenced by UspA during the state of fungal developmental competence and up to $24 \mathrm{~h}$ of multicellular development. This indicates that CsnE is no target for UspA polyubiquitin chain removal. It cannot be excluded that UspA might influence the stability of other COP9 signalosome components.

A. nidulans strains deficient in any of the eight CSN subunits exhibit pleiotropic phenotypes; increased initiation of the sexual life cycle with the inability to form mature cleistothecia is concomitant with an altered secondary metabolism (Beckmann et al., 2015; Busch et al., 2003, 2007). The gene deletion of $u s p A$ has rather mild effects on fungal development, whereby it supports the formation of conidiospores as well as the timely coordination of sexual fruiting body formation. In case the COP9 signalosome subunits would be a target for the deubiquitinase, the uspA deletion would be expected to exhibit much stronger phenotypes that resemble csn deletion developmental defects. UspA might use the COP9 signalosome as surface to reach its target proteins for deubiquitination reactions (Figure 46).

This was shown for the tumor suppressor protein adenomatous polyposis coli (APC), the inhibitor protein IKBa for the mammalian NF-KB transcription factor family and for the binding partners of the ATPase valosin-containing protein (p97/VCP), which ubiquitination states are dependent on Usp15 (Cayli et al., 2009; Huang et al., 2009; Schweitzer et al., 2007). 


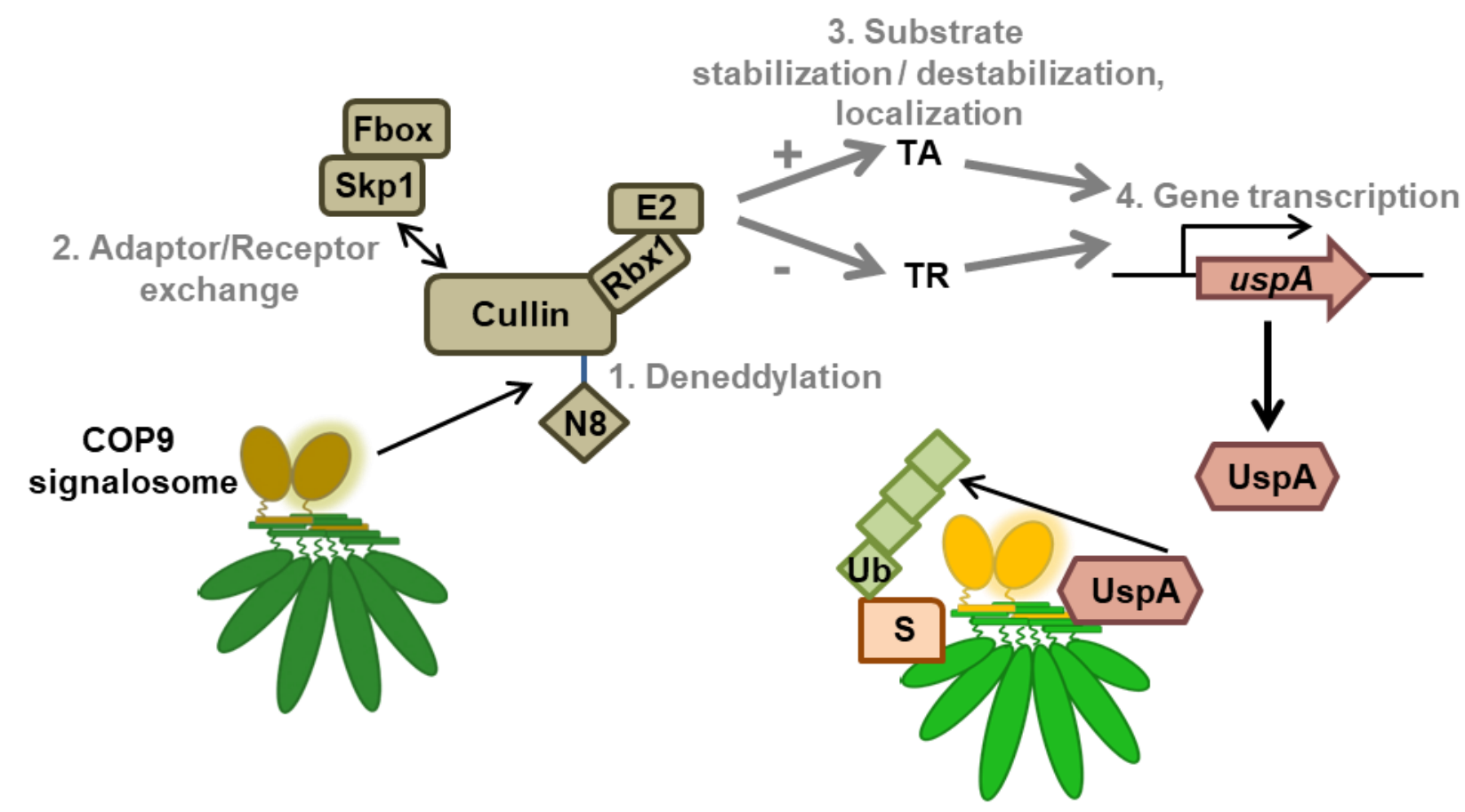

Figure 46: UspA might interact with the COP9 signalosome during the catalysis of deubiquitinating reactions.

The transcript levels of uspA are repressed in the presence of a functional COP9 signalosome during multicellular development. UspA interacts with subunits of the COP9 signalosome in vivo and in vitro. For orthologs in other organisms like humans, deubiquitination of substrates takes place while the DUB interacts with the COP9 signalosome.

\subsection{UspA reduces the cellular pool of ubiquitinated proteins during multicellular fungal development}

Deubiquitinating enzymes remove ubiquitin molecules or chains from modified proteins (Komander et al., 2009). Many proteins are labeled with ubiquitin chains prior to their degradation by the $26 \mathrm{~S}$ proteasome (Glickman and Ciechanover, 2002). Deletion of the uspA encoding gene or the expression of an inactive UspA mutant protein leads to drastic elevation of proteins modified with ubiquitin in total cell extracts during multicellular development in A. nidulans (Figure 28). Compared to the rather mild effects of a non-functional UspA protein on fungal multicellular development, a drastic increase in ubiquitinated proteins in total protein extracts was observed. Even though uspA deficient strains have a normal vegetative growth phenotype, the amount of ubiquitinated proteins already increases significantly during the state of developmental competence. The $26 \mathrm{~S}$ proteasome might constantly degrade labeled proteins, but cannot process the substrates for degradation as fast so that the ubiquitinated proteins are 
accumulating. Another possible scenario would be that the accumulating proteins are labeled with ubiquitin molecules or chains that do not target proteins for degradation by the $26 \mathrm{~S}$ proteasome.

USPs often have overlapping ubiquitin-chain linkage specificity and might have overlapping substrate spectra as deletion studies of USP encoding genes in $S$. pombe and $S$. cerevisiae revealed no severe growth phenotypes (Amerik et al., 2000; Kouranti et al., 2010). An overlapping function of another fungal USP would also counteract the accumulation of ubiquitinated proteins observed in $u s p A$ deficient strains. At the moment it remains still elusive, if ubiquitin chains of a certain linkage type accumulate in these strains or wether rather monoubiquitinated substrates accumulate.

Taken together, UspA allows through its deubiquitination reactions the timely coordination of events that are necessary for multicellular development and coordinated secondary metabolism in $A$. nidulans.

The identification of SM gene clusters in strains with defects in the ubiquitin-proteasome system was already a successful method in the past (Gerke et al., 2012). Thereby, $\Delta c s n E$ was utilized in the identification of the $d b a$ gene cluster (Gerke et al., 2012). The requirement of a functional COP9 signalosome for coordinated secondary metabolism was already indicated by the regulation of the transcript levels of several genes belonging to different clusters as well (Nahlik et al., 2010). A. nidulans deletion strains of uspA or other deubiquitinating enzymes might pave the way for future identification of so far unkown secondary metabolites or intermediates.

\subsubsection{UspA cleaves polyubiquitin chains that are bound to substrates}

The velocity of catalytic reactions in biological systems is drastically increased through the mechanism of enzymes (Robinson, 2015). Enzymes bind to specific substrates, catalyze the reaction and release the modified substrate (Robinson, 2015). Due to the high speed of the reaction it is often difficult to identify specific substrates of certain enzymes. One way to overcome this issue is to construct catalytically inactive enzyme mutants, which are supposed to bind longer or stronger to the substrate, but are not able to enhance substrate turnover. The mammalian ubiquitin-specific protease Usp3 targets histones $\mathrm{H} 2 \mathrm{~A}$ and $\mathrm{H} 2 \mathrm{~B}$ for deubiquitination (Nicassio et al., 2007). This discovery is based on mutant Usp3 proteins, which carried amino acid exchanges of a histidine or a cysteine residue, respectively, which rendered the protein inactive and lead to co-purification of ubiquitinated H2A. Functional Usp3 did not co-purify the histone proteins (Nicassio et al., 2007). 
GFP pull down experiments with the functional UspA-GFP fusion protein and the inactive $U_{s p A^{A A}-G F P}$ version were performed with the aim to identify potential targets for deubiquitination. The interactome (the entirety of the putative interaction partners) of UspA is mostly independent from its catalytic activity.

The identification of ubiquitin in the GFP pull down with the inactive UspA mutant suggests that UspA might not bind to the substrate proteins directly, but rather interacts with the polyubiquitin chains. It might be involved in the recycling of free polyubiquitin chains, which is necessary to provide a free ubiquitin pool for subsequent ligation reactions (Komander et al., 2009). Human USPs involved in the supply of the free ubiquitin molecule pool due to cleavage of free polyubiquitin chains, like Usp5, contain a specific zinc finger ubiquitin-binding domain ZnF-UBP motif (Dayal et al., 2009; Reyes-Turcu et al., 2006). Human Usp15, Usp11 and Usp4 lack this ZnF-UBP motif (Komander et al., 2009). This indicates that UspA rather attacks polyubiquitin chains bound to substrates instead of free polyubiquitin chains.

The cleavage of ubiquitin molecules from a polyubiquitin chain does not necessarily require the direct binding of the DUB to the substrate (Komander et al., 2009). Exo- or endo deubiquitinases bind directly to the ubiquitin chain, whereas DUBs removing monoubiquitin will come into direct proximity to the substrate (Komander et al., 2009). Linkage specific DUBs such as mammalian CYLD have two binding sites for the proximal and distal ubiquitin and do not need to have direct contact to the ubiquitinated protein (Sato et al., 2015; Schaefer and Morgan, 2011). The crystal structure of the Usp7 catalytic domain revealed a right-handed architecture consisting of palm, thumb and fingers, which can efficiently bind ubiquitin (Hu et al., 2002).

\subsection{The localization and putative interaction partners of UspA suggest a role in controlling nuclear transport processes}

UspA-GFP is localized primarily at the nuclear periphery and subpopulations exist in the nuclei and in the cytoplasm during vegetative growth (Figure 47). BiFC and co-localization experiments with the $\mathrm{PCl}$ domain containing $\mathrm{CSN}$ subunit CsnB revealed that mostly the nuclear fraction of UspA interacts with the COP9 signalosome. The catalytic activity of UspA influences the localization slightly; the inactive mutant protein accumulates still close to nuclei and shows a population that is distributed through the cytoplasm. An A. nidulans CsnD-GFP fusion was previously observed in the cytoplasm and accumulates in nuclei (Busch et al., 2003). The catalytic active subunit Csn5/E localizes predominantly inside the nucleus when it is 
incorporated into the COP9 signalosome (Tomoda et al., 2002). Single Csn5/E or smaller subcomplexes were also detected in the cytoplasm. Csn5 carries an NES, which serves as recognition factor for the export factor CRM1 (Tomoda et al., 2002).

The human Usp15 and its paralogous proteins Usp11 and Usp4 are all at least partially localized inside nuclei (Sowa et al., 2009; Vlasschaert et al., 2015). Usp4 is mainly localized in the nucleus, but excluded from the nucleolus, whereas its paralog Usp15 shows preferably cytoplasmic or nucleolus localization in NIH3T3 or HeLa cells (Soboleva et al., 2005). Consistently, the $S$. pombe ortholog Ubp12 has a cytoplasmic and nuclear subpopulation (Kouranti et al., 2010). The ubiquitin-specific protease UBP109 is the rat ortholog of human Usp15 (Park et al., 2000). Three nuclear localization sequences were recognized in its sequence, whereas only one influences subcellular localization (Park et al., 2000). This NLS motif with the sequence - LKKR - is also present in human Usp15. In silico analysis of the amino acid sequence of UspA with the NucPred Software was performed, which calculates the likelihood for every single amino acid to be involved in nuclear localization (Brameier et al., 2007). It revealed a NLS with very high scores for the amino acid sequence "RRKKK", a region close to its C-terminus spanning residues 1034-1038. This region is identified as potential NLS also with the software cNLS Mapper with a score of 3.5 (Kosugi et al., 2009). This score is rather low and means that the protein is localized in both, cytoplasm and nucleus. This result fits well to the observed localization pattern of UspA in A. nidulans. UspA-GFP as well as UspA ${ }^{\mathrm{AA}}$-GFP pull numerous proteins related to nuclear transport, like the kayopherins KapB and KapF (Figure 47). Whereas KapF is localized in nuclei, KapB was observed at the nuclear periphery and both proteins are essential for fungal life (Markina-Inarrairaegui et al., 2011). Nucleocytoplasmic shuttling activity was attributed to the Usp4 ortholog in mice (Soboleva et al., 2005). Mice Usp4 contains NLS as well as NES sequences and migrates into the nucleus with the importin $\alpha / \beta$. In general, DUBs can be localized in the nucleus or in the cytoplasm and can regulate nuclear transport of proteins. This study provides evidence that in the filamentous fungus $A$. nidulans, where several nuclei can be part of one cellular compartement, the deubiquitinase UspA is pimarily localized in close proximity to nuclei. Due to its putative NLS and putative interaction partners that are involved in nuclear transport, it is reasonable to suggest a function of the deubiquitinase in nuclear transport.

Besides the localization of DUBs itself, they can be involved in the regulation of subcellular localization of other proteins by removing ubiquitination patterns of certain lysine residues in the target protein. The subcellular localization of the phosphatase and tensin homolog (PTEN), which functions as tumor suppressor, is influenced by monoubiquitination events in cancer cell 
lines (Trotman et al., 2007). Furthermore, deubiquitination of PTEN ensures the destination inside the nucleus. Deubiquitinated PTEN is supposed to perform its function in the nucleus, whereas ubiquitinated forms will be shuttled to the cytoplasm. In this example, ubiquitination of certain lysine residues directly affects cellular localization (Trotman et al., 2007). It is possible that UspA is involved in such a regulatory mechanism where the substrates of deubiquitination are still unknown.

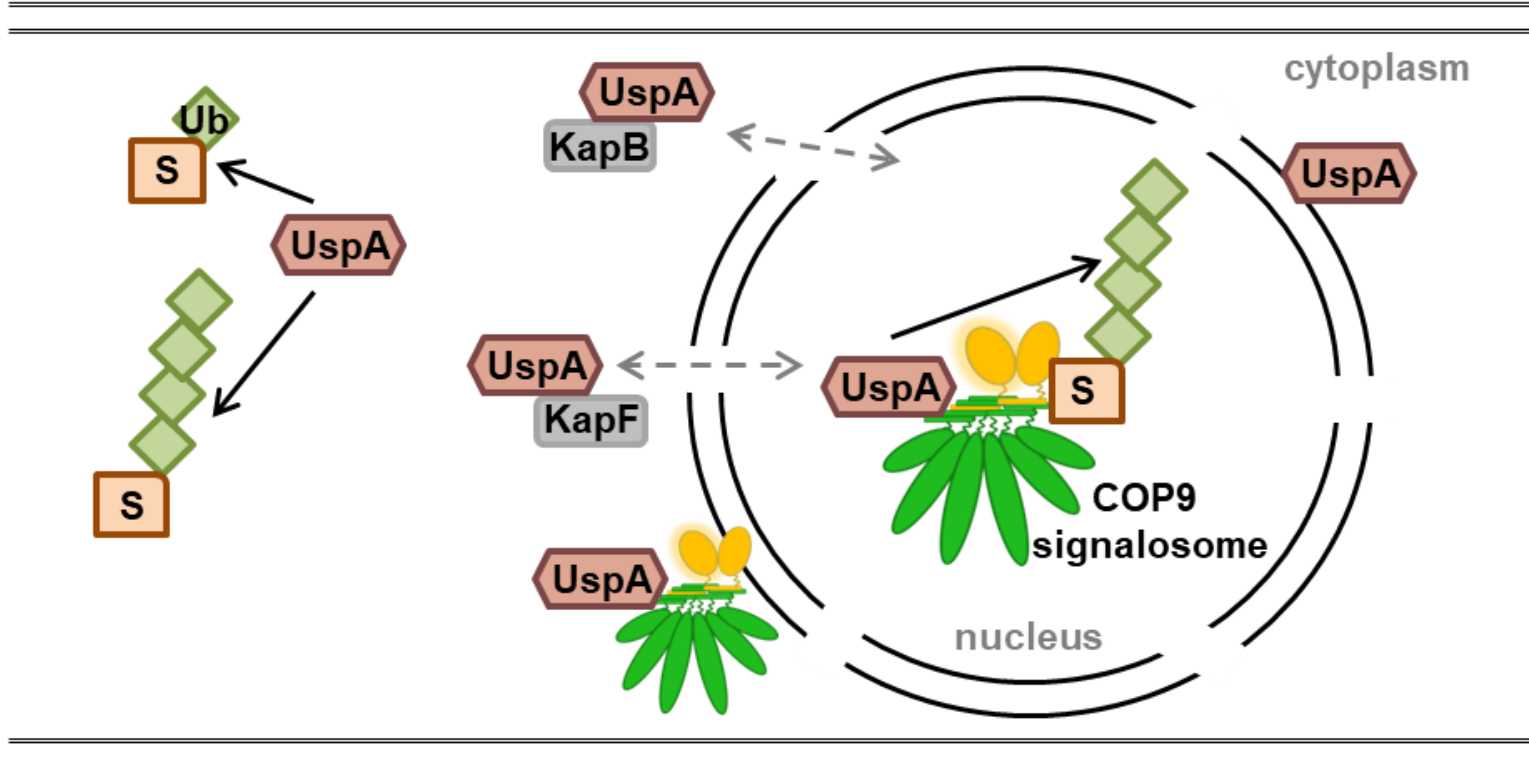

Figure 47: UspA accumulates primarily close to nuclei and appears in smaller subpopulations inside the nucleus as well as in the cytoplasm.

UspA removes ubiquitin (Ub) molecules from substrates (S) and accumulates close to nuclei. It mainly interacts with subunits of the COP9 signalosome inside the nucleus, but a subpopulation is also located at the nuclear periphery. UspA interacts with nuclear import factors, which might recognize the putative NLS located in the C-terminal part of UspA and might be involved in translocation of the deubiquitinase into the nucleus.

Furthermore, the active and the inactive UspA fusion protein versions pulled eleven proteins related to transcriptional processing altogether. Pull downs of the UspA protein suggest a function of the deubiquitinase in transcriptional elongation as the active UspA-GFP pulled two Polymerase II transcription elongation factors and two proteins, which regulate the transcription. The inactive UspA ${ }^{A A}$-GFP pulled a putative subunit of the Ccr4-Not complex. BLAST analyses revealed that the pulled protein AN4965 has highest similarities with the subunit Not1 of this protein complex, the scaffolding protein. This Ccr4-Not1 complex has several functions in gene transcription and translation processes (Collart, 2016). Usp4 and Usp15 were suggested to have 
a role in RNA processing in humans as they associate to members of the splicing machinery (Sowa et al., 2009). Usp11 rather associates to different transcription elongation factors (Sowa et al., 2009).

Taken together, the function of fungal and mammalian USP orthologs is quite conserved. Usp15, Usp4 and Usp11 interact with proteins related to transcription and translation processes or with proteins that are involved in RNA processing steps (Song et al., 2010; Sowa et al., 2009). The A. nidulans ortholog UspA pulls several proteins related to this processes in GFP pull down experiments as well.

\subsection{UspA regulates the protein abundance of the major developmental regulator VeA and early multicellular development of $\boldsymbol{A}$. nidulans}

VeA is an important regulator of development in A. nidulans (Bayram et al., 2008b; Bayram and Braus, 2012). Its gene deletion leads to an inability to form cleistothecia, whereas its overexpression leads to constant sexual fruiting body formation independently of light (Kim et al., 2002). VeA and the COP9 signalosome have antagonistic function in fungal development: $\Delta v e A$ does not develop any cleistothecia even in darkness, whereas csn mutants initiate fruiting body formation even in light (Beckmann et al., 2015; Busch et al., 2003, 2007; Kim et al., 2002). Deletion strains of $v e A$ or csn subunits are not able to form mature cleistothecia (Beckmann et al., 2015; Busch et al., 2003, 2007; Kim et al., 2002). The velvet protein family is well conserved in filamentous fungi and well-studied in the phylum of ascomycetes (Bayram and Braus, 2012; Gerke and Braus, 2014; $\mathrm{Ni}$ and $\mathrm{Yu}, 2007)$. The VeA ortholog in Aspergillus parasiticus is required for the formation of the resting structure, called sclerotia, as well (Calvo et al., 2004). Furthermore, VeA in Aspergillus flavus is required for sclerotia formation (Duran et al., 2007). An impact of VeA on conidiospore formation was recently reported in Aspergillus niger (Wang et al., 2015). In A. fumigatus $\mathrm{VeA}$ is required for conidiation as well (Krappmann et al., 2005). Co-cultivation of different $A$. nidulans veA mutant strains with larvae of $D$. melanogaster revealed an effect of the functional VeA protein on the development of the fruit fly (Regulin and Kempken, 2018).

Even though ubiquitinated proteins accumulate in total fungal crude extracts during all developmental stages of $A$. nidulans in uspA mutant strains, the effect of the deubiquitinase on the phenotype under the tested conditions is rather mild. A reduction in conidiospore formation, 
a delay in sexual fruiting body formation and an altered secondary metabolism was observed in $\Delta u s p A$ or a strain expressing an inactive UspA mutant.

This study shows that the deubiquitinase UspA influences the abundance of the VeA-GFP fusion protein. UspA does not stabilize VeA-GFP during growth in asexual or sexual development inducing conditions. In the absence of UspA, VeA-GFP fusion protein accumulates after $24 \mathrm{~h}$ of development. This indicates rather an indirect effect of UspA on the velvet domain containing protein VeA. If UspA targets K48 linked ubiquitin chains, which sentence substrates for proteasomal degradation, VeA would be destabilized and degraded in the absence of the deubiquitinase. Therefore, UspA might deubiquitinate another protein, which in turn is able to regulate the protein levels of $\mathrm{VeA}$.

Kim and co-workers postulated that $\mathrm{VeA}$ acts as a negative regulator during asexual conidiospore formation (Kim et al., 2002). They observed more conidiophores in a veA deletion strain than in the wild type (Kim et al., 2002). VeA has a repressing effect on the transcript levels of the major asexual development transcriptional regulator brlA (Kato et al., 2003). Additionally, the deletion of the ve-1 ortholog in $N$. crassa leads to increased conidiation of the fungus (Bayram et al., 2008c). During this study, increased amounts of VeA-GFP protein during ongoing asexual development might be the reason for the observed reduction in the amount of conidiospores.

The veA gene is expressed during vegetative growth and during the whole asexual and sexual development (Kim et al., 2002). This indicates that the changes in the fusion protein abundance shown in this study are rather taking place on protein level probably caused by posttranslational modifications and not on gene expression level. In turn, the changed fusion protein abundance can be admitted to protein stability that is among other factors regulated by deubiquitinating enzymes.

The lack of higher molecular weight bands of VeA-GFP on the western hybridization, which could represent ubiquitinated forms of $\mathrm{VeA}$, might be explained by the direct turnover and degradation of VeA-GFP through the $26 \mathrm{~S}$ proteasome. The human ortholog of UspA, Usp15, is able to cleave K48 and K63 polyubiquitin chains, which are well-known to send substrates for proteasomal degradation (Cornelissen et al., 2014). A decrease in VeA-GFP protein abundance would be expected in $\triangle u s p A$, if UspA would protect VeA-GFP for proteasomal degradation during fungal development. The deubiquitinase might not affect $\mathrm{VeA}$ directly, but might regulate stability or activity of for example a kinase, which in turn regulates VeA stability (Figure 48). 


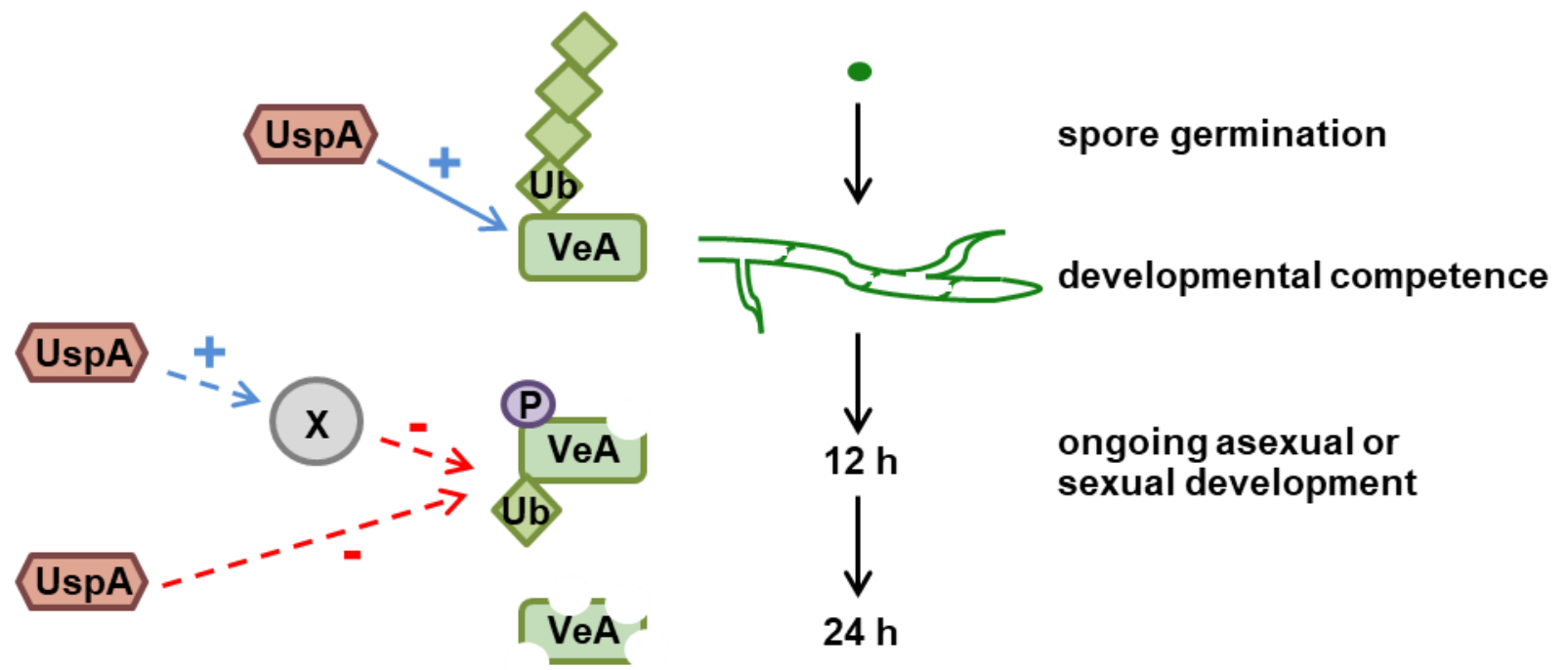

Figure 48: Putative modes of regulation of VeA through the deubiquitinase UspA.

At the stage of developmental competence UspA might stabilize VeA protein abundance. During ongoing growth in asexual or sexual development inducing conditions, VeA abundance decreases. This might be due to direct or indirect action of UspA. UspA might stabilize a kinase or phosphatase $(X)$ through its deubiquitination activity, which in turn modifies VeA phosphorylation $(P)$ pattern and regulates its stability. UspA might also regulate for example $\mathrm{VeA}$ monoubiquitination ( $\mathrm{Ub}$ ) and influence thereby its function, abundance or interaction network.

Due to the presence of two PEST domains in the amino acid sequence of VeA it is a target for phosphorylation events, which influence its protein stability (Bayram et al., 2012; Rauscher et al., 2016; Rechsteiner and Rogers, 1996; Rogers et al., 1986). Kinases are regulated by ubiquitination in diverse manner (Ball et al., 2016). Besides the polyubiquitination of kinases, which leads to their degradation, ubiquitin or other UBL proteins can regulate kinase activity without changing their stability (Ball et al., 2016; Mohapatra et al., 2008). Similar regulatory effects on phosphatases have been shown as well (Yang et al., 2010). The phosphatase PTEN is important for tumor suppression and its intracellular localization depends on its monoubiquitination (Trotman et al., 2007). If similar mechanisms are true for VeA protein abundance still remains to be experimentally verified.

The phenotype of the $\triangle u s p A^{p y r o A}$ strain was more pronounced when the strain was grown on medium that was not supplemented with pyridoxine. The conidiospore amount was reduced to only $15 \%$ compared to the number of wild type spores and after seven days of growth in sexual development inducing conditions no mature cleistothecia have been observed. The complementation strain, which was also grown in the absence of pyridoxine, could restore the wild type phenotype completely. The pyroA gene, used as marker cassette for construction of this deletion strain, is essential for the biosynthesis of pyridoxine (Osmani et al., 1999). This 
gene enables $A$. nidulans to produce pyridoxine again. One might argue that the pyro $A$ gene becomes not transcribed in wild type like amounts as it is not expressed at its own gene locus, but under the control of the uspA promotor. However, if this would be true, a development inducing effect should be observed for the complementation strain comp ${ }^{\text {pyroA }}$ after addition of pyridoxine as well, which is not the case. There might be a crosstalk between the deubiquitinase and the pyridoxine synthesis pathway, while stability of proteins required for pyridoxine biosynthesis are regulated by ubiquitination or deubiquitination reactions. No experimental evidence exists so far for all these hypotheses and they stay a matter of investigation. Independently of the used marker cassette, the accumulation of ubiquitinated proteins during all developmental stages of $A$. nidulans, a retardation of sexual fruiting body development and an alteration in secondary metabolism were clearly assigned to the function of UspA in this study.

\subsubsection{VeA is a target for posttranslational modifications}

$\mathrm{VeA}$ is phosphorylated at several sites and this influences function or the localization of the protein (Rauscher et al., 2016). Two of the identified phosphorylation sites have an impact on fungal development and protein localization. The mutant expressing constantly dephosphorylated T167 by mutation of threonine to valine and a mimic of constantly phosphorylated T170 by mutation to glutamic acid showed the most severe effects. VeA ${ }^{\text {T167VT170E }}$ showed increased gene expression, impairments in the interaction with VelB and shows a similar phenotype to the veA gene deletion (Rauscher et al., 2016).

So far, there is no experimental evidence of VeA ubiquitination sites. The amino acid sequence of VeA encompasses 32 lysine residues, which are putative attachment sites for posttranslational modifications like ubiquitin. The online ubiquitination site prediction tools Ubpred and UbiSite were used to predict putative modification sites in VeA (Huang et al., 2016; Radivojac et al., 2011). Putative modified lysine residues of VeA are depicted in Table 18.

The prediction of the two online tools differ quite heavily: only two sites were predicted by both tools. The most promising lysine residue for putative ubiquitination events, which would be promising to address in future experiments, is K509. 
Table 18: Ubiquitination site prediction for VeA.

The VeA amino acid sequence was applied to the online tools UbPred and UbiSite to calculate probabilities for the different lysine residues to be ubiquitinated (Huang et al., 2016; Radivojac et al., 2011). Only lysine residues with medium or high confidence are given. Only K107 and K509 were predicted by both prediction tools to be ubiquitination sites of $\mathrm{VeA}$ in $A$. nidulans.

\begin{tabular}{|c|c|c|}
\hline Residue & UbPred & UbiSite \\
\hline K80 & - & medium confidence \\
\hline K107 & medium confidence & medium confidence \\
\hline K161 & - & high confidence \\
\hline K164 & - & medium confidence \\
\hline K180 & - & medium confidence \\
\hline K205 & - & medium confidence \\
\hline K241 & medium confidence & - \\
\hline K459 & medium confidence & - \\
\hline K509 & high confidence & medium confidence \\
\hline K562 & - & medium confidence \\
\hline
\end{tabular}

\subsubsection{Velvet domain proteins have similarities to the mammalian NF-kB transcription factor family}

Besides the already mentioned $\mathrm{VeA}$, the conserved fungal velvet protein family consists of three additional members in A. nidulans, namely VelB, VosA and VelC (Bayram and Braus, 2012; Gerke and Braus, 2014; Ni and Yu, 2007). Velvet proteins share the name-giving approximately 150 amino acid long velvet domain, which serves as protein-protein interaction domain (Ahmed et al., 2013; Bayram and Braus, 2012). All four proteins form diverse homo- or heterodimers, or trimeric protein complexes with each other and also with other proteins (Figure 49). They react on external stimuli such as light or temperature and migrate into the nucleus. Nuclear entry of velvet proteins is under tight control through many other proteins, such as the methyltransferases VapB/VipC or LImF (Figure 49) (Palmer et al., 2013; Sarikaya-Bayram et al., 2014, 2015). Velvet proteins regulate fungal development and the closely connected secondary metabolism (Sarikaya-Bayram et al., 2014, 2015). One function of velvet domain containing proteins is the regulation of secondary metabolite production, which can help the fungus to resist against predators or harsh environmental conditions. Velvet domain proteins are able to bind DNA and influence gene transcription (Ahmed et al., 2013). The understanding of how velvet domain proteins regulate fungal secondary metabolism in detail might lead to identification of so far unknown secondary metabolites, which might be of industrial or medical use for humankind. 
Similarly, NF-kB proteins are a mammalian transcription factor family, which is well conserved in different cell types and tissues (Oeckinghaus and Ghosh, 2009). The protein family consists of five members: p65 (RelA), RelB, c-Rel, p105/p50 (NF-kB1) and p100/52 (NF-kB2). Similar to the velvet domain proteins they are able to form different homo- or heterodimers (Oeckinghaus and Ghosh, 2009). A characteristic feature of this protein family is the Rel homology domain (RHD), which encompasses approximately 300 amino acids and mediates protein-protein interaction and DNA binding (Baldwin, 1996). These proteins react on external stimuli such as exposure to tumor necrosis factor $\alpha$ (TNFa) or $\underline{B}$-cell activating factor (BAFF). Exposure to these stimuli leads to phosphorylation of IKBa or the RelB specific inhibitor Rel100. As a consequence thereof they become a substrate for UPS (Gilmore, 2006; Sun, 2011). Through binding of IkBa to the COP9 signalosome, which in turn interacts with Usp15, the inhibitor becomes stabilized again approximately $60 \mathrm{~min}$ after stimuli exposure (Guardavaccaro et al., 2003; Schweitzer et al., 2007).

Analysis of crystal structures of the velvet and the RHD domain revealed a high structural similarity (Ahmed et al., 2013). Velvet domains are a characteristic protein family in filamentous fungi, whereas the NF-kB protein family was exclusively identified in mammals. Capsaspora owczarzaki, a unicellular eukaryote, carries one protein with a velvet domain and another one with a Rel homology domain (Ahmed et al., 2013). This suggests a common evolutionary origin of both protein families and explains the similarities among both systems.

Fbox proteins $\beta \operatorname{TrCP} 1$ and $\beta$ TrCP2 are responsible for the targeted ubiquitination and concomitant degradation of IKBa (Guardavaccaro et al., 2003). Protein BLAST analyses of the amino acid sequences of $\beta$ TrCP1 (Uniprot ID: Q9Y297) and $\beta T r C P 2$ (Uniprot ID: Q9UKB1) against the Aspergillus nidulans database revealed Fbox protein 23 among the best hits (E value: $4 \mathrm{e}^{-43}$ or $3 \mathrm{e}^{-43}$ ), respectively. SCF complexes bound to Fbox23 or Fbox15 accumulate in strains harboring a defective COP9 signalosome (von Zeska Kress et al., 2012). Fbox23 is required for repression of sexual development during light conditions. A. nidulans $\Delta f b o x 23$ develops increased numbers of cleistothecia under illumination (von Zeska Kress et al., 2012). This mimics the phenotype of a veA overexpression strain, which already form Hülle cells and cleistothecia in liquid cultures and under illumination when the wild type forms only a few sexual fruiting bodies (Kim et al., 2002). Wether Fbox23 influences the stability of VeA and wether Fbox23 is a substrate of the deubiquitinating enzyme UspA needs to be investigated in future studies. 


\section{fungal velvet domain family}

light, temperature,

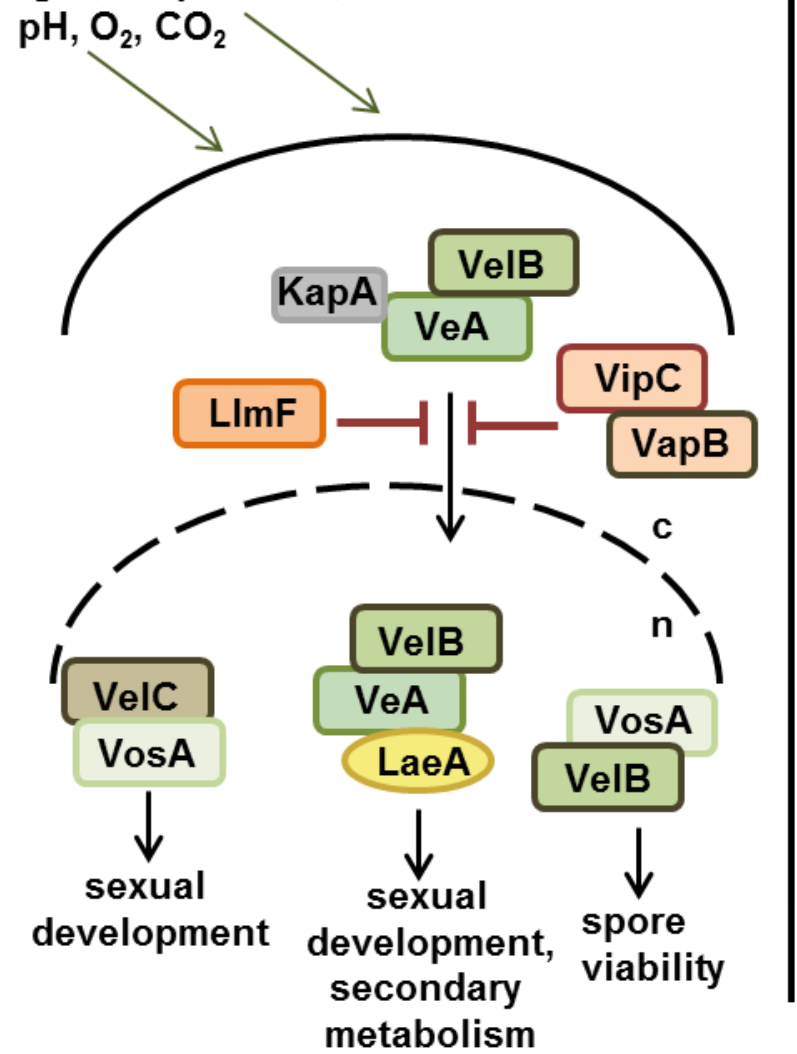

mammalian Rel homology domain family
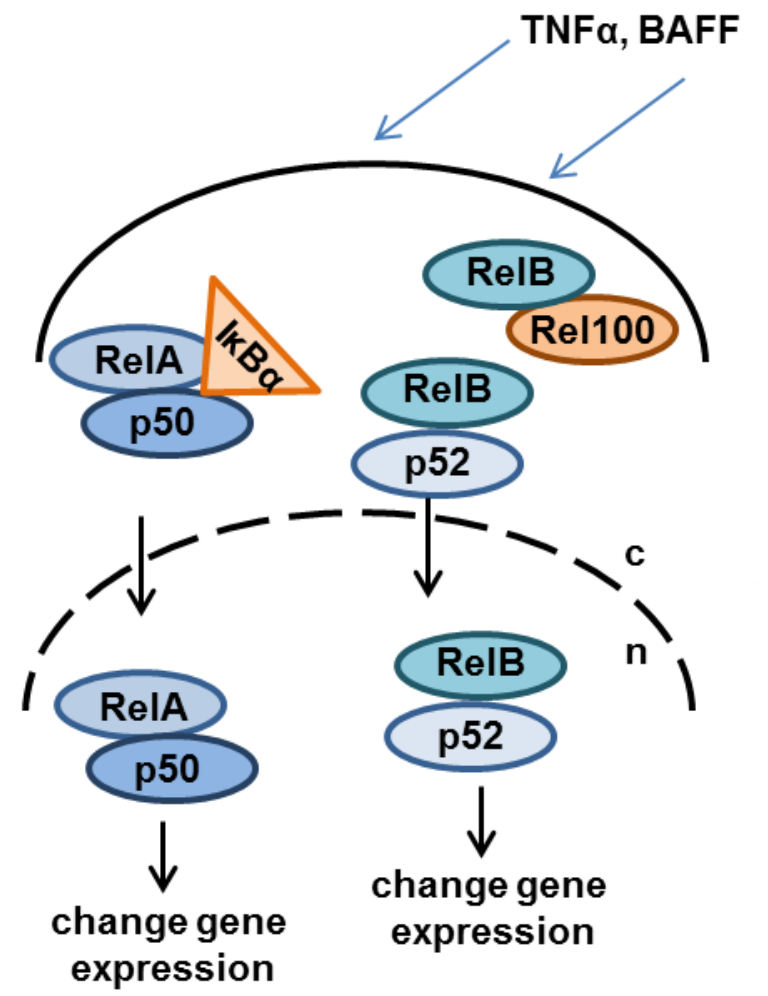

Figure 49: Fungal velvet domain proteins show high similarities to the mammalian NF-kB RHD domain containing transcription factor family.

Due to external stimuli fungal velvet domain containing proteins as well as mammalian NF-KB transcription factors migrate from the cytoplasm (c) into the nucleus ( $\mathrm{n}$ ) to change gene expression directly or indirectly. Both protein families form diverse homo- and heterodimeric complexes. Fungi react on external stimuli like light, temperature, $\mathrm{pH}$, oxygen and carbon dioxide concentrations, whereas Rel homology domain proteins react on stimuli like tumor necrosis factor 1 (TNF1) or B-cell activating factor (BAFF) (Bayram et al., 2008b; Gilmore, 2006; Sarikaya-Bayram et al., 2014). 


\subsection{Conclusion and outlook}

Posttranslational modifications of proteins can change their function, localization, interaction partners or stability (Duan and Walther, 2015). The modification of proteins with ubiquitin is versatile due to the different ubiquitin chain linkage types and their corresponding function (Ohtake and Tsuchiya, 2017; Pickart and Eddins, 2004). Proteins modified with K48 linked ubiquitin chains are targeted for the degradation by the 26S protesome (Finley et al., 1994; Spasser and Brik, 2012). The present study provides insights into different levels of regulation of this ubiquitin-proteasome system. The dysfunction or dysregulation of the UPS is the cause of many diseases (Paul, 2008).

The COP9 signalosome influences the labeling of substrates with ubiquitin due to the deneddylation activity towards the cullin subunit of CRLs (Beckmann et al., 2015; Lyapina et al., 2001). It binds CRLs that are not associated to substrates and enables due to the induction of conformational changes the exchange of the receptor complexes. This ensures specific substrate binding (Choo et al., 2011; Mosadeghi et al., 2016). Here, SILAC was established for the use in $A$. nidulans to quantitatively examine the changes in the proteome in the absence of a functional COP9 signalosome. Already during the vegetative growth phase, CsnE influences abundances of 99 proteins. Thereby, many developmental proteins involved in hyphal growth or in the formation of septa are stabilized by CsnE. Alternatively, CsnE mainly reduces abundances of proteins involved in primary metabolism (Figure 50) In future studies, the proteome during A. nidulans multicellular development in csnE mutants can be analyzed. The L-lysine and L-arginine auxotrophic strains generated in the scope of this study are the basis for future quantitiative analyses of proteins in $A$. nidulans.

The second level of the ubiquitin-proteasome system regulation is conferred by the function of deubiquitinating enzymes, which remove ubiquitin chains from target proteins (Komander et al., 2009). This study provides an overview about the different DUB families and their members in A. nidulans. The ortholog of human Usp15, UspA, interacts with subunits of the COP9 signalosome in vivo and in vitro. A dysfunctional COP9 signalosome by deletion of CsnE encoding gene leads to an upregulation of $u s p A$ transcript levels during multicellular development. Considering the high similarities to the mammalian system, UspA might deubiquitinate substrates that are bound to the COP9 signalosome. A summarizing model depicts the interconnection between the COP9 signalosome deneddylase and the UspA deubiquitinase (Figure 50). 


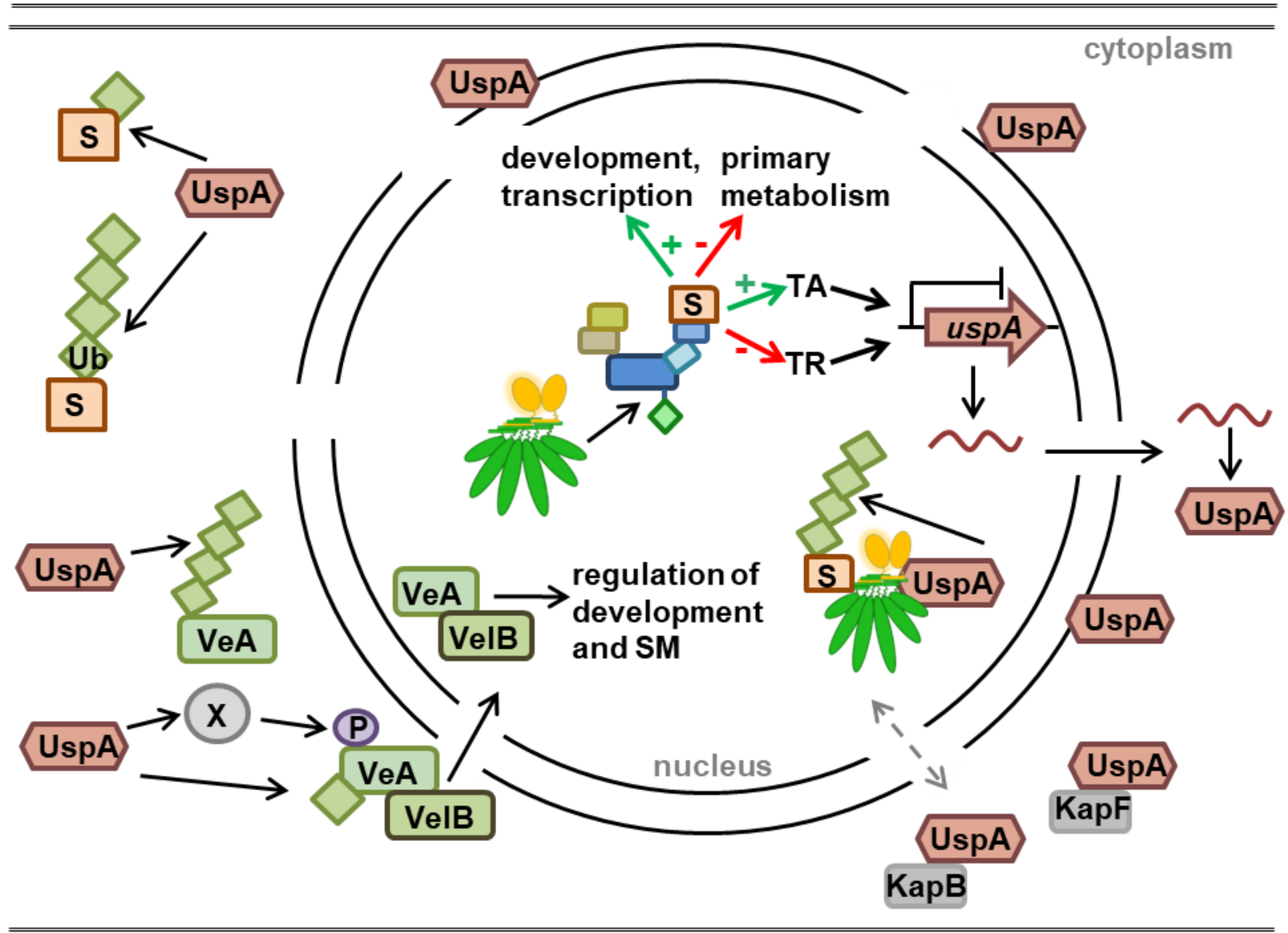

Figure 50: Model of the interplay between the COP9 signalosome deneddylase and the UspA deubiquitinase.

The COP9 signalosome (CSN) differentially regulates the abundance of proteins related to primary metabolism, fungal development and transcriptional regulation. By influencing the abundance of a transcriptional activator (TA) or transcriptional repressor (TR) it represses the transcription levels of uspA. Once transcribed, uspA mRNA might be transported to the cytoplasm and translated into a functional protein. UspA can associate with nuclear transport factors KapB and KapF and enter the nucleus again to interact with the COP9 signalosome. UspA is responsible for the removal of ubiquitin $(\mathrm{Ub})$ molecules or chains from a broad range of substrates (S) during all developmental stages of $A$. nidulans. Thereby, it influences directly or indirectly the protein abundance of $\mathrm{VeA}$, a major regulator of fungal development and secondary metabolism. UspA might deubiquitinate $\mathrm{VeA}$ directly or influence the protein abundance of another protein $(X)$ such as a kinase or a phosphatase, which in turn change the phosphorylation $(P)$ pattern of $\mathrm{VeA}$, which in turn alters its function. VeA ensures a timely coordinated order of events during fungal multicellular development.

UspA reduces the pool of ubiquitinated proteins in the fungal cell during all developmental stages. The substrates for deubiquitination are not identified yet. Therefore, ubiquitinated proteins have to be enriched in an uspA deficient strain and analyzed with LC/MS-MS in future 
studies. Furthermore, the novel established SILAC method for $A$. nidulans is suitable to investigate protein stability in dependeny of the dubiquitinating enzyme. DUBs are proposed to regulate protein stability by removing the ubiquitin chains prior to the degradation of the protein through the $26 \mathrm{~S}$ proteasome. Therefore, the substrates of UspA deubiquitination are likely changed in their abundance in an $u s p A$ deficient strain. Additionally, the ubiquitin chain hydrolyzing activity of recombinantly purified UspA towards differently linked, chemically synthezied ubiquitin chains can be tested to shed some light on UspA specific substrates.

UspA changes the protein abundance of the major fungal regulator of development and secondary metabolism VeA. This is accompanied by a delay in sexual development and an altered secondary metabolism. Fungal velvet proteins are structurally very similar to mammalian NF-kB transcription factors. The function of mammalian NF-kBs is regulated by deubiquitination reactions of their inhibitor proteins like IKBa through Usp15. The strong structural and functional conservation of this system underlines its importance for life. It needs to be addressed in future studies wether VeA is a direct deubiquitination target of UspA or wether another protein such as a kinase or phosphatase is the deubiquitination target, which in turn regulates the abundance of VeA.

Taken together, this study provides insights into two distinct but connected levels of regulation of the ubiquitin-proteasome system. The interconnection of the COP9 signalosome deneddylase and UspA deubiquitinase are important for timely coordinated multicellular development and secondary metabolism in $A$. nidulans. A broad overview about $A$. nidulans DUBs provides the basis for future studies to shed light on the function of these enzymes. The novel established SILAC method will thereby be an excellent method to identify substrates of DUBs. 


\section{References}

Abdul Rehman, S.A., Kristariyanto, Y.A., Choi, S., Nkosi , P.J., Weidlich, S., Labib, K., Hofmann, K. and Kulathu, Y., (2016). MINDY-1 is a member of an evolutionarily conserved and structurally distinct new family of deubiquitinating enzymes. Mol Cell. $\underline{63}, 146-155$.

Adams, T.H., Boylan, M.T. and Timberlake, W.E., (1988). brlA is necessary and sufficient to direct conidiophore development in Aspergillus nidulans. Cell. $\underline{54}$, 353-362.

Adams, T.H., Wieser, J.K. and Yu, J.H., (1998). Asexual sporulation in Aspergillus nidulans. Microbiol Mol Biol Rev. $\underline{62}, 35-54$.

Adnan, M., Zheng, W., Islam, W., Arif, M., Abubakar, Y.S., Wang, Z. and Lu, G., (2018). Carbon catabolite repression in filamentous fungi. Int J Mol Sci. 19, doi: 10.3390/ijms19010048.

Aebersold, R., (2003). Quantitative proteome analysis: methods and applications. J Infect Dis. 187, S315-S320.

Aggarwal, K. and Massagué, J., (2012). Ubiquitin removal in the TGF- $\beta$ pathway. Nat Cell Biol. $\underline{14}, 656-657$.

Ahmed, Y.L., Gerke, J., Park, H.S., Bayram, Ö., Neumann, P., Ni, M., Dickmanns, A., Kim, S.C., Yu, J.H., Braus, G.H. and Ficner, R., (2013). The velvet family of fungal regulators contains a DNA-binding domain structurally similar to NF-KB. PLoS Biol. 11, doi: 10.1371/journal.pbio. 1001750 .

Al-Salihi, M.A., Herhaus, L. and Macartney, T., (2012). USP11 augments TGF- $\beta$ signalling by deubiquitylating ALK5. Open Biol. 2, doi: 10.1098/rsob.120063.

Alam, M.A. and Kelly, J.M., (2017). Proteins interacting with CreA and CreB in the carbon catabolite repression network in Aspergillus nidulans. Curr Genet. $\underline{63}, 669-683$.

Altschul, S.F., Gish, W., Miller, W., Myers, E.W. and Lipman, D.J., (1990). Basic local alignment search tool. J Mol Biol. 215, 403-410.

Amerik, A.Y. and Hochstrasser, M., (2004). Mechanism and function of deubiquitinating enzymes. Biochim Biophys Acta - Mol Cell Res. 1695, 189-207.

Amerik, A.Y., Li, S. and Hochstrasser, M., (2000). Analysis of the deubiquitinating enzymes of the yeast Saccharomyces cerevisiae. Biol Chem. 381, 981-992.

Aramayo, R., Adams, T.H. and William, E., (1989). A large cluster of highly expressed genes is dispensable for growth and development in Aspergillus nidulans. Genetics. 122, 65-71.

Arst, H.N. Jr., (1977). Some genetical aspects of ornithine metabolism in Aspergillus nidulans. Mol Gen Genet. 151, 105-110.

Axelrod, D., Gealt, M. and Pastushok, M., (1973). Gene control of developmental competence in Aspergillus nidulans. Dev Biol. 34, 9-15. 
Bah, A. and Forman-Kay, J.D., (2016). Modulation of intrinsically disordered protein function by post-translational modifications. J Biol Chem. 291, 6696-6705.

Baker, R.T., Wang, X., Woollatt, E., White, J.A. and Sutherland, G.R., (1999). Identification, functional characterization, and chromosomal localization of USP15, a novel human ubiquitin-specific protease related to the UNP oncoprotein, and a systematic nomenclature for human ubiquitin-specific proteases. Genomics. $\underline{59}$, 264-274.

Baldwin, A.S., (1996). The NF-KB and IKB proteins: New discoveries and insights. Annu Rev Immunol. 14, 649-681.

Ball, K.A., Johnson, J.R., Lewinski, M.K., Guatelli, J., Verschueren, E., Krogan, N.J. and Jacobson, M.P., (2016). Non-degradative ubiquitination of protein kinases. PLoS Comput Biol. 12, doi: 10.1371/journal.pcbi.1004898.

Ballario, P., Vittorioso, P., Magrelli, A., Talora, C., Cabibbo, A., Macino, G. and Sapienza, L., (1996). White collar-1, a central regulator of blue light responses in Neurospora, is a zinc finger protein. EMBO J. 15, 1650-1657.

Bayram, Ö., Bayram, Ö.S., Ahmed, Y.L., Maruyama, J., Valerius, O., Rizzoli, S.O., Ficner, R., Irniger, S. and Braus, G.H., (2012). The Aspergillus nidulans MAPK module AnSte11Ste50-Ste7-Fus3 controls development and secondary metabolism. PLoS Genet. $\underline{8}$, doi: 10.1371/journal.pgen.1002816.

Bayram, Ö., Biesemann, C., Krappmann, S., Galland, P. and Braus, G.H., (2008a). More than a repair enzyme: Aspergillus nidulans photolyase-like CryA is a regulator of sexual

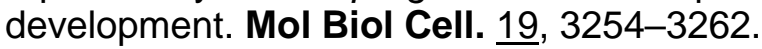

Bayram, Ö. and Braus, G.H., (2012). Coordination of secondary metabolism and development in fungi: The velvet family of regulatory proteins. FEMS Microbiol Rev. $\underline{36}$, doi: 10.1111/j.1574-6976.2011.00285.x.

Bayram, Ö., Braus, G.H., Fischer, R. and Rodriguez-Romero, J., (2010). Spotlight on Aspergillus nidulans photosensory systems. Fungal Genet Biol. $\underline{47}$, 900-908.

Bayram, Ö., Feussner, K., Dumkow, M., Herrfurth, C., Feussner, I. and Braus, G.H., (2016). Changes of global gene expression and secondary metabolite accumulation during

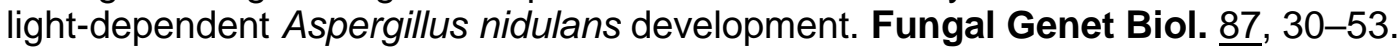

Bayram, Ö., Krappmann, S., Ni, M., Bok, J.W., Helmstaedt, K., Valerius, O., Braus-Stromeyer, S., Kwon, N.J., Keller, N.P., Yu, J.H. and Braus, G.H., (2008b). VelB/VeA/LaeA complex coordinates light signal with fungal development and secondary metabolism. Science. $\underline{320}$, 1504-1506.

Bayram, Ö., Krappmann, S., Seiler, S., Vogt, N. and Braus, G.H., (2008c). Neurospora crassa ve-1 affects asexual conidiation. Fungal Genet Biol. $\underline{45}$, 127-138.

Bech-Otschir, D., Kraft, R., Huang, X., Henklein, P., Kapelari, B., Pollmann, C. and Dubiel, W., (2001). COP9 signalosome-specific phosphorylation targets p53 to degradation by the ubiquitin system. EMBO J. 므, 1630-1639. 
Beckmann, E.A., Köhler, A.M., Meister, C., Christmann, M., Draht, O.W., Rakebrandt, N., Valerius, O. and Braus, G.H., (2015). Integration of the catalytic subunit activates deneddylase activity in vivo as final step in fungal COP9 signalosome assembly. Mol Microbiol. $\underline{97}, 110-124$.

Beltrao, P., Bork, P., Krogan, N.J. and van Noort, V., (2013). Evolution and functional cross-talk of protein post-translational modifications. Mol Syst Biol. $\underline{9}$, doi: 10.1002/msb.201304521.

Bennett, J.W., (1998). Mycotechnology: The role of fungi in biotechnology. J. Biotechnol. $\underline{66}$, 101-107.

Bergkessel, M. and Guthrie, C., (2013). Colony PCR. In: Methods Enzymol. pp. 299-309.

Bertani, G., (1951). Studies on lysogenesis. I. The mode of phage liberation by lysogenic Escherichia coli. J Bacteriol. 62, 293-300.

Bishop, P., Rocca, D. and Henley, J.M., (2016). Ubiquitin C-terminal hydrolase L1 (UCH-L1): structure, distribution and roles in brain function and dysfunction. Biochem J. $\underline{473}, 2453-$ 2462.

Blumenstein, A., Vienken, K., Tasler, R., Purschwitz, J., Veith, D., Frankenberg-Dinkel, N. and Fischer, R., (2005). The Aspergillus nidulans phytochrome FphA represses sexual development in red light. Curr Biol. 15, 1833-1838.

Bok, J.W. and Keller, N.P., (2004). LaeA, a regulator of secondary metabolism in Aspergillus spp. Eukaryot. Cell. $\underline{3}, 527-535$.

Bok, J.W., Noordermeer, D., Kale, S.P. and Keller, N.P., (2006). Secondary metabolic gene cluster silencing in Aspergillus nidulans. Mol Microbiol. 61, 1636-1645.

Bosu, D.R. and Kipreos, E.T., (2008). Cullin-RING ubiquitin ligases: global regulation and activation cycles. Cell Div. $\underline{3}$, doi: 10.1186/1747-1028-3-7.

Brakhage, A.A., (1998). Molecular regulation of beta-lactam biosynthesis in filamentous fungi. Microbiol Mol Biol Rev. $\underline{62}, 547-585$.

Brakhage, A.A., (2012). Regulation of fungal secondary metabolism. Nat Rev Microbiol. 11, 21-32.

Brameier, M., Krings, A. and MacCallum, R.M., (2007). NucPred - Predicting nuclear localization of proteins. Bioinformatics. $\underline{23}, 1159-1160$.

Braus, G., Krappmann, S. and Eckert, S., (2002). Sexual development in ascomycetes: Fruit body formation of Aspergillus nidulans. In: Osiewacz, H.D. (Ed.), Molecular Biology of Fungal Development. CRC Press, New York, pp. 215-244.

Braus, G.H., Irniger, S. and Bayram, Ö., (2010). Fungal development and the COP9 signalosome. Curr Opin Microbiol. $13,672-676$. 
Bremm, A. and Komander, D., (2011). Emerging roles for Lys11-linked polyubiquitin in cellular regulation. Trends Biochem Sci. $\underline{36}$, 355-363.

Brown, D.W. and Salvo, J.J., (1994). Isolation and characterization of sexual spore pigments from Aspergillus nidulans. Appl Environ Microbiol. 60, 979-983.

Busch, S., Eckert, S.E., Krappmann, S. and Braus, G.H., (2003). The COP9 signalosome is an essential regulator of development in the filamentous fungus Aspergillus nidulans. Mol Microbiol. $\underline{49}, 717-730$.

Busch, S., Schwier, E.U., Nahlik, K., Bayram, Ö., Helmstaedt, K., Draht, O.W., Krappmann, S., Valerius, O., Lipscomb, W.N. and Braus, G.H., (2007). An eight-subunit COP9 signalosome with an intact JAMM motif is required for fungal fruit body formation. Proc Natl Acad Sci U S A. $\underline{104}, 8089-8094$.

Bylebyl, G.R., Belichenko, I. and Johnson, E.S., (2003). The SUMO isopeptidase Ulp2 prevents accumulation of SUMO chains in yeast. J Biol Chem. 278, 44113-44120.

Cadwell, K., Coscoy, L., Martin, G.B. and Stebbins, C.E., (2005). Ubiquitination on nonlysine residues by a viral E3 ubiquitin ligase. Science. $\underline{309}, 127-130$.

Calvo, A.M., Bok, J., Brooks, W., Keller, P. and Keller, N.P., (2004). veA is required for toxin and sclerotial production in Aspergillus parasiticus. Appl Environ Microbiol. 0ㅡ, 4733-4739.

Calvo, A.M., Wilson, R.A., Bok, J.W. and Keller, N.P., (2002). Relationship between secondary metabolism and fungal development. Microbiol Mol Biol Rev. $\underline{66}, 447-459$.

Cao, J. and Yan, Q., (2012). Histone ubiquitination and deubiquitination in transcription, DNA damage response, and cancer. Front Oncol. 2, doi: 10.3389/fonc.2012.00026.

Casadevall, A., (2012). Fungi and the rise of mammals. PLoS Pathog. $\underline{8}$, doi: 10.1371/journal.ppat.1002808.

Casadevall, A., Fang, F.C. and Pirofski, L.-A., (2011). Microbial virulence as an emergent property: Consequences and opportunities. PLoS Pathog. $\underline{7}$, doi: 10.1371/journal.ppat.1002136.

Casselton, L. and Zolan, M., (2002). The art and design of genetic screens: filamentous fungi. Nat Rev Genet. $\underline{3}$, 683-697.

Cayli, S., Klug, J., Chapiro, J., Fröhlich, S., Krasteva, G., Orel, L. and Meinhardt, A., (2009). COP9 signalosome interacts ATP-dependently with p97/valosin-containing protein (VCP) and controls the ubiquitination status of proteins bound to p97/VCP. J Biol Chem. 284, 34944-34953.

Cerqueira, G.C., Arnaud, M.B., Inglis, D.O., Skrzypek, M.S., Binkley, G., Simison, M., Miyasato, S.R., Binkley, J., Orvis, J., Shah, P., Wymore, F., Sherlock, G. and Wortman, J.R., (2014). The Aspergillus Genome Database: Multispecies curation and incorporation of RNA-Seq data to improve structural gene annotations. Nucleic Acids Res. $\underline{42}, 705-710$. 
Chan, Y., Yoon, J., Wu, J.T., Kim, H.J., Pan, K.T., Yim, J. and Chien, C.T., (2008). DEN1 deneddylates non-cullin proteins in vivo. J Cell Sci. 121, 3218-3223.

Chenna, R., Sugawara, H., Koike, T., Lopez, R., Gibson, T.J., Higgins, D.G. and Thompson, J.D., (2003). Multiple sequence alignment with the Clustal series of programs. Nucleic Acids Res. $\underline{31}, 3497-3500$.

Chiu, Y.H., Xiang, X., Dawe, A.L. and Morris, N.R., (1997). Deletion of nudC, a nuclear migration gene of Aspergillus nidulans, causes morphological and cell wall abnormalities and is lethal. Mol Biol Cell. $\underline{8}, 1735-1749$.

Choo, Y.Y., Boh, B.K., Lou, J.J., Eng, J., Leck, Y.C., Anders, B., Smith, P.G. and Hagen, T., (2011). Characterization of the role of COP9 signalosome in regulating cullin E3 ubiquitin ligase activity. Mol Biol Cell. 22, 4706-4715.

Christmann, M., Schmaler, T., Gordon, C., Huang, X., Stumpf, S., Dubiel, W. and Braus, G.H., (2013). Control of multicellular development by the physically interacting deneddylases DEN1/DenA and COP9 signalosome. PLoS Genet. $\underline{9}$, doi: 10.1371/journal.pgen.1003275.

Ciechanover, A., Orian, A. and Schwartz, A.L., (2000). Ubiquitin-mediated proteolysis: biological regulation via destruction. Bioessays. $\underline{22}, 442-451$.

Clague, M.J., Barsukov, I., Coulson, J.M., Liu, H., Rigden, D.J. and Urbé, S., (2013).

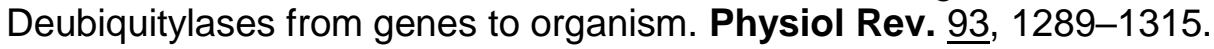

Clague, M.J., Coulson, J.M. and Urbé, S., (2012). Cellular functions of the DUBs. J Cell Sci. $\underline{125}, 277-286$.

Clutterbuck, A.J., (1969). A mutational analysis of conidial development in Aspergillus nidulans. Genetics. $\underline{63}, 317-327$.

Colabardini, A.C., Humanes, C.A., Gouvea, P.F., Goldman, M.H., von Zeska Kress, M.R., Bayram, Ö., Oliviera, J.V., Gomes, M.D., Braus, G.H. and Goldman, G.H., (2012). Molecular characterization of the Aspergillus nidulans $f b x A$ encoding an F-box protein involved in xylanase induction. Fungal Genet Biol. $\underline{49}$, 130-140.

Collart, M.A., (2016). The Ccr4-Not complex is a key regulator of eukaryotic gene expression. Wiley Interdiscip Rev RNA. 7 , 438-454.

Collier, T.S., Hawkridge, A.M., Georgianna, D.R., Payne, G.A. and Muddiman, D.C., (2013). Top-Down identification and quantification of stable isotope labeled proteins from $A$. flavus using online nano-flow reversed phase liquid chromatography coupled to a LTQ-FT-ICR mass spectrometer. Anal Chem. $\underline{80}$, 4994-5001.

Collinge, A.J. and Markham, P., (1985). Woronin bodies rapidly plug septal pores of severed chrysogenum hyphae. Exp Mycol. $\underline{9}, 80-85$.

Cope, G.A. and Deshaies, R.J., (2003). COP9 signalosome: A multifunctional regulator of SCF and other cullin-based ubiquitin ligases. Cell. 114, 663-671. 
Cope, G.A. and Deshaies, R.J., (2006). Targeted silencing of Jab1/Csn5 in human cells downregulates SCF activity through reduction of F-box protein levels. BMC Biochem. $\underline{7}$, doi: 10.1186/1471-2091-7-1.

Cope, G.A., Suh, G.S., Aravind, L., Schwarz, S.E., Zipursky, S.L., Koonin, E. V and Deshaies, R.J., (2002). Role of predicted metalloprotease motif of Jab1/Csn5 in cleavage of Nedd8 from Cul1. Science. $\underline{298}$, 608-612.

Cornelissen, T., Haddad, D., Wauters, F., Van Humbeeck, C., Mandemakers, W., Koentjoro, B., Sue, C., Gevaert, K., De Strooper, B., Verstreken, P. and Vandenberghe, W., (2014). The deubiquitinase USP15 antagonizes Parkin-mediated mitochondrial ubiquitination and mitophagy. Hum Mol Genet. 23, 5227-5242.

Cox, J. and Mann, M., (2008). MaxQuant enables high peptide identification rates, individualized p.p.b.-range mass accuracies and proteome-wide protein quantification. Nat Biotechnol. 26, 1367-1372.

Craig, K.L. and Tyers, M., (1999). The F-box: a new motif for ubiquitin dependent proteolysis in cell cycle regulation and signal transduction. Prog Biophys Mol Biol. $\underline{72}$, 299-328.

Danielsen, J.M., Sylvestersen, K.B., Bekker-Jensen, S., Szklarczyk, D., Poulsen, J.W., Horn, H., Jensen, L.J., Mailand, N. and Nielsen, M.L., (2011). Mass spectrometric analysis of lysine ubiquitylation reveals promiscuity at site level. Mol Cell Proteomics. $\underline{10}$, doi: M110.003590.

Dayal, S., Sparks, A., Jacob, J., Allende-Vega, N., Lane, D.P. and Saville, M.K., (2009). Suppression of the deubiquitinating enzyme USP5 causes the accumulation of unanchored polyubiquitin and the activation of p53. J Biol Chem. 284, 5030-5041.

de Godoy, L.M., Olsen, J.V., de Souza, G.A., Li, G., Mortensen, P. and Mann, M., (2006). Status of complete proteome analysis by mass spectrometry: SILAC labeled yeast as a model system. Genome Biol. ㄱ, doi: 10.1186/gb-2006-7-6-r50.

de Jong, R.N., Eiso A.B., Diercks, T., Truffault, V., Daniëls, M., Kaptein, R. and Folkers, G.E., (2006). Solution structure of the human ubiquitin-specific protease 15 DUSP domain. J Biol Chem. 281, 5026-5031.

Dean, R., Van Kan, J.A., Pretorius, Z.A., Hammond-Kosack, K.E., Di Pietro, A., Spanu, P.D., Rudd, J.J., Dickman, M., Kahmann, R., Ellis, J. and Foster, G.D., (2012). The Top 10 fungal pathogens in molecular plant pathology. Mol Plant Pathol. 13, 414-430.

de Poot, S.A.H., Tian, G. and Finley, D., (2017). Meddling with fate: the proteasomal deubiquitinating enzymes. J Mol Biol. $\underline{429}$, 3525-3545.

de Vries, R.P., Riley, R., Wiebenga, A., Aguilar-Osorio, G., Amillis, S., Uchima, C.A., Anderluh, G., Asadollahi, M., Askin, M., Barry, K., Battaglia, E., Bayram, Ö., Benocci, T., BrausStromeyer, S.A., Caldana, C., Cánovas, D., Cerqueira, G.C., Chen, F., Chen, W., Choi, C., Clum, A., Dos Santos, R.A.C., Damásio, A.R., Diallinas, G., Emri, T., Fekete, E., Flipphi, M., Freyberg, S., Gallo, A., Gournas, C., Habgood, R., Hainaut, M., Harispe, M.L., Henrissat, B., Hildén, K.S., Hope, R., Hossain, A., Karabika, E., Karaffa, L., Karányi, Z., 
Kraševec, N., Kuo, A., Kusch, H., LaButti, K., Lagendijk, E.L., Lapidus, A., Levasseur, A., Lindquist, E., Lipzen, A., Logrieco, A.F., MacCabe, A., Mäkelä, M.R., Malavazi, I., Melin, P., Meyer, V., Mielnichuk, N., Miskei, M., Molnár, Á.P., Mulé, G., Ngan, C.Y., Orejas, M., Orosz, E., Ouedraogo, J.P., Overkamp, K.M., Park, H.S., Perrone, G., Piumi, F., Punt, P.J., Ram, A.F.J., Ramón, A., Rauscher, S., Record, E., Riaño-Pachón, D.M., Robert, V., Röhrig, J., Ruller, R., Salamov, A., Salih, N.S., Samson, R.A., Sándor, E., Sanguinetti, M., Schütze, T., Sepčić, K., Shelest, E., Sherlock, G., Sophianopoulou, V., Squina, F.M., Sun, H., Susca, A., Todd, R.B., Tsang, A., Unkles, S.E., van de Wiele, N., van Rossen-Uffink, D., Oliveira, J.V., Vesth, T.C., Visser, J., Yu, J.H., Zhou, M., Andersen, M.R., Archer, D.B., Baker, S.E., Benoit, I., Brakhage, A.A., Braus, G.H., Fischer, R., Frisvad, J.C., Goldman, G.H., Houbraken, J., Oakley, B., Pócsi, I., Scazzocchio, C., Seiboth, B., vanKuyk, P.A., Wortman, J., Dyer, P.S. and Grigoriev, I.V, (2017). Comparative genomics reveals high biological diversity and specific adaptations in the industrially and medically important fungal genus Aspergillus. Genome Biol. 18,.doi: 10.1186/s13059-017-1151-0.

Deng, L., Wang, C., Spencer, E., Yang, L., Braun, A., You, J., Slaughter, C., Pickart, C. and Chen, Z.J., (2000a). Activation of the IkB kinase complex by TRAF6 requires a dimeric ubiquitin-conjugating enzyme complex and a unique polyubiquitin chain. Cell. $\underline{103}$, 351361.

Deng, X.W., Dubiel, W., Wei, N., Hofmann, K., Mundt, K., Naumann, M., Segal, D., Seeger, M., Carr, A., Glickman, M. and Chamovitz, D.A., (2000b). Unified nomenclature for the COP9 signalosome and its subunits: an essential regulator of development. Trends Genet. $\underline{16}$, 202-203.

Deshaies, R.J. and Joazeiro, C.A., (2009). RING domain E3 ubiquitin ligases. Annu Rev Biochem. 78, 399-434.

Dohmann, E.M., Levesque, M.P., De Veylder, L., Reichardt, I., Jürgens, G., Schmid, M. and Schwechheimer, C., (2008). The Arabidopsis COP9 signalosome is essential for G2 phase progression and genomic stability. Development. 135, 2013-2022.

Drocourt, D., Calmels, T., Reynes, J.P., Baron, M. and Tiraby, G., (1990). Cassettes of the Streptoalloteichus hindustanus ble gene for transformation of lower and higher eukaryotes to phleomycin resistance. Nucleic Acids Res. $\underline{18}, 4009$.

Duan, G. and Walther, D., (2015). The roles of post-translational modifications in the context of protein interaction networks. PLoS Comput Biol. 11, doi: 10.1371/journal.pcbi.1004049.

Dubiel, D., Rockel, B., Naumann, M. and Dubiel, W., (2015). Diversity of COP9 signalosome structures and functional consequences. FEBS Lett. $\underline{589}$, 2507-2513.

Duda, D.M., Borg, L.A., Scott, D.C., Hunt, H.W., Hammel, M. and Schulman, B.A., (2008). Structural insights into NEDD8 activation of cullin-RING ligases: conformational control of conjugation. Cell. 134, 995-1006.

Duran, R.M., Cary, J.W. and Calvo, A.M., (2007). Production of cyclopiazonic acid, aflatrem, and aflatoxin by Aspergillus flavus is regulated by $v e A$, a gene necessary for sclerotial formation. Appl Microbiol Biotechnol. 3ㅡ, 1158-1168. 
Dyer, P.S. and O'Gorman, C.M., (2011). Sexual development and cryptic sexuality in fungi: insights from Aspergillus species. FEMS Microbiol Rev. $\underline{36}$, 165-192.

Eletr, Z.M. and Wilkinson, K.D., (2014). Regulation of proteolysis by human deubiquitinating enzymes. Biochim Biophys Acta. 1843, 114-128.

Enchev, R.I., Scott, D.C., da Fonseca, P.C., Schreiber, A., Monda, J.K., Schulman, B.A., Peter, M. and Morris, E.P., (2012). Structural basis for a reciprocal regulation between SCF and CSN. Cell Rep. $2,616-627$.

Etxebeste, O., Herrero-García, E., Cortese, M.S., Garzia, A., Oiartzabal-Arano, E., de los Ríos, V., Ugalde, U. and Espeso, E.A., (2012). GmcA is a putative glucose-methanol-choline oxidoreductase required for the induction of asexual development in Aspergillus nidulans. PLoS One. $\underline{7}$, doi: 10.1371/journal.pone.0040292.

Fan, J.B., Arimoto, K.L., Motamedchaboki, K., Yan, M., Wolf, D.A. and Zhang, D.E., (2015). Identification and characterization of a novel ISG15-ubiquitin mixed chain and its role in regulating protein homeostasis. Sci Rep. $\underline{5}$, doi: 10.1038/srep12704.

Faronato, M., Urbé, S. and Coulson, J., (2011). USP15 (ubiquitin specific peptidase 15). Atlas Genet Cytogenet Oncol Haematol. 15, 645-651.

Feldman, R.M., Correll, C.C., Kaplan, K.B. and Deshaies, R.J., (1997). A complex of Cdc4p , Skp1p, and Cdc53p/Cullin catalyzes ubiquitination of the phosphorylated CDK Inhibitor

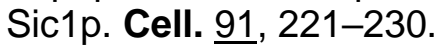

Finley, D., Sadis, S., Monia, B.P., Boucher, P., Ecker, D.J., Crooke, S.T. and Chau, V., (1994). Inhibition of proteolysis and cell cycle progression in a multiubiquitination-deficient yeast mutant. Mol Cell Biol. 14, 5501-5509.

Finley, D., Ulrich, H.D., Sommer, T. and Kaiser, P., (2012). The ubiquitin-proteasome system of Saccharomyces cerevisiae. Genetics. 192, 319-360.

Flick, J.S. and Johnston, M., (1990). Two systems of glucose repression of the GAL1 promoter in Saccharomyces cerevisiae. Mol Cell Biol. 10, 4757-4769.

Frias-Staheli, N., Giannakopoulos, N.V., Kikkert, M., Taylor, S.L., Bridgen, A., Paragas, J., Richt, J.A., Rowland, R.R., Schmaljohn, C.S., Lenschow, D.J., Snijder, E.J., García-Sastre, A. and Virgin, H.W. 4th, (2007). Ovarian tumor domain-containing viral proteases evade ubiquitinand ISG15-dependent innate immune responses. Cell Host Microbe. $\underline{2}$, 404-416.

Froehlich, A.C., Liu, Y. and Loros, J.J., (2002). White Collar-1, a circadian blue light photoreceptor, binding to the frequency promoter. Science. 297, 815-819.

Galagan, J.E., Calvo, S.E., Cuomo, C., Ma, L., Wortman, J.R., Batzoglou, S., Spevak, C.C., Clutterbuck, J., Kapitonov, V., Jurka, J., Scazzocchio, C., Farman, M., Butler, J., Purcell, S., Harris, S., Braus, G.H., Draht, O., Busch, S., Enfert, C.D., Bouchier, C., Goldman, G.H., Denning, D.W., Caddick, M., Hynes, M., Paoletti, M., Fischer, R., Miller, B., Dyer, P., Sachs, M.S., Osmani, S.A. and Birren, B.W. (2005). Sequencing of Aspergillus nidulans and comparative analysis with $A$. fumigatus and A. oryzae. Nature. $\underline{438}$, 1105-1115. 
Gan-Erdene, T., Nagamalleswari, K., Yin, L., Wu, K., Pan, Z.Q. and Wilkinson, K.D., (2003). Identification and characterization of DEN1, a deneddylase of the ULP family. J Biol Chem. 278, 28892-28900.

Gerke, J., Bayram, Ö., Feussner, K., Landesfeind, M., Shelest, E., Feussner, I. and Braus, G.H., (2012). Breaking the silence: protein stabilization uncovers silenced biosynthetic gene clusters in the fungus Aspergillus nidulans. Appl Environ Microbiol. $\underline{78}$, 8234-8244.

Gerke, J. and Braus, G.H., (2014). Manipulation of fungal development as source of novel secondary metabolites for biotechnology. Appl Microbiol Biotechnol. 묘, 8443-8455.

Gilmore, T.D., (2006). Introduction to NF-kB: players, pathways, perspectives. Oncogene. $\underline{25}$, $6680-6684$

Glickman, M.H. and Ciechanover, A., (2002). The ubiquitin-proteasome proteolytic pathway: Destruction for the sake of construction. Physiol Rev. $\underline{82}$, 373-428.

Goldenberg, S.J., Cascio, T.C., Shumway, S.D., Garbutt, K.C., Liu, J., Xiong, Y., Zheng, N., Hill, C. and Carolina, N., (2004). Structure of the Cand1-Cul1-Roc1 complex reveals regulatory mechanisms for the assembly of the multisubunit cullin-dependent ubiquitin ligases. Cell. $119,517-528$.

Golemis, E.A., Serebriiskii, I., Finley, R.L., Kolonin, M.G., Gyuris, J. and Brent, R., (2001). Interaction trap/two-hybrid system to identify interacting proteins. Curr Protoc Cell Biol. 17, doi:10.1002/0471143030.cb1703s08.

Golemis, E.A., Serebriiskii, I. and Law, S.F., (1999). The yeast two-hybrid system: criteria for detecting physiologically significant protein-protein interactions. Curr Issues Mol Biol. 1 , $31-45$.

Grant, S.G., Jessee, J., Bloom, F.R. and Hanahan, D., (1990). Differential plasmid rescue from transgenic mouse DNAs into Escherichia coli methylation-restriction mutants. Proc Natl Acad Sci U S A. 87 , 4645-4649.

Grou, C.P., Pinto, M.P., Mendes, A.V, Domingues, P. and Azevedo, J.E., (2015). The de novo synthesis of ubiquitin: identification of deubiquitinases acting on ubiquitin precursors. Sci Rep. $\underline{5}$, doi: 10.1038/srep12836.

Gruhler, A., Olsen, J.V., Mohammed, S., Mortensen, P., Færgeman, N.J., Mann, M. and Jensen, O.N., (2005). Quantitative phosphoproteomics applied to the yeast pheromone signaling pathway. Mol Cell Proteomics. 4, 310-327.

Guardavaccaro, D., Kudo, Y., Boulaire, J., Barchi, M., Busino, L., Donzelli, M., Margottin-Goguet, F., Jackson, P.K., Yamasaki, L. and Pagano, M., (2003). Control of meiotic and mitotic progression by the F box protein $\beta-T r c p 1$ in vivo. Dev Cell. 4 , 799-812.

Gygi, S.P., Corthals, G.L., Zhang, Y., Rochon, Y. and Aebersold, R., (2000). Evaluation of two-dimensional gel electrophoresis-based proteome analysis technology. Proc Natl Acad

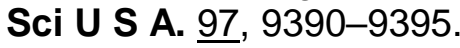


Han, K.H., Chun, Y.H., Figueiredo, Bde. C., Soriani, F.M., Savoldi, M., Almeida, A., Rodrigues, F., Cairns, C.T., Bignell, E., Tobal, J.M., Goldman, M.H., Kim, J.H., Bahn, Y.S., Goldman, G.H. and Ferreira, M.E., (2010). The conserved and divergent roles of carbonic anhydrases in the filamentous fungi Aspergillus fumigatus and Aspergillus nidulans. Mol Microbiol. $\underline{75}$, $1372-1388$.

Hanpude, P., Bhattacharya, S., Dey, A.K. and Maiti, T.K., (2015). Deubiquitinating enzymes in cellular signaling and disease regulation enzymatic mechanism of DUBs. IUBMB Life. $\underline{67}$, $544-555$.

Harding, R.W. and Melles, S., (1983). Genetic analysis of phototropism of Neurospora crassa perithecial beaks using white collar and albino mutants. Plant Physiol. 르, 996-1000.

Harper, S., Besong, T.M., Emsley, J., Scott, D.J. and Dreveny, I., (2011). Structure of the USP15 $\mathrm{N}$-terminal domains: a $\beta$-hairpin mediates close association between the DUSP and UBL

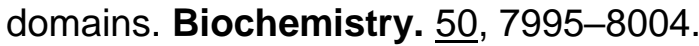

Harris, S.D., (2001). Septum formation in Aspergillus nidulans. Curr Opin Microbiol. 4, 736739.

Harris, S.D., (2008). Branching of fungal hyphae: regulation, mechanisms and comparison with other branching systems branching systems. Mycologia. $\underline{100}$, 823-832.

Harris, S.D., Turner, G., Meyer, V., Espeso, E.A., Specht, T., Takeshita, N. and Helmstedt, K., (2009). Morphology and development in Aspergillus nidulans: a complex puzzle. Fungal Genet Biol. 뜨, S82-S92.

Hartmann-Petersen, R., Hendil, K.B., Gordon, C. and Kutay, U., (2003). Ubiquitin binding proteins protect ubiquitin conjugates from disassembly. FEBS Lett. $\underline{535}, 77-81$.

Hartmann, M., Heinrich, G. and Braus, G.H., (2001). Regulative fine-tuning of the two novel DAHP isoenzymes aroFp and aroGp of the filamentous fungus Aspergillus nidulans. Arch Microbiol. 175, 112-121.

Hartmann, T., Dümig, M., Jaber, B.M., Szewczyk, E., Olbermann, P., Morschhäuser, J. and Krappmann, S., (2010). Validation of a self-excising marker in the human pathogen Aspergillus fumigatus by employing the $\beta$-Rec/six site-specific recombination system. Appl Env Microbiol. 76, 6313-6317.

Hatfield, P.M., Gosink, M.M., Carpenter, T.B. and Vierstra, R.D., (1997). The ubiquitin-activating enzyme (E1) gene family in Arabidopsis thaliana. Plant J. 11, 213-226.

Hay-Koren, A., Caspi, M., Zilberberg, A. and Rosin-Arbesfeld, R., (2011). The EDD E3 ubiquitin ligase ubiquitinates and up-regulates $\beta$-catenin. Mol Biol Cell. 22, 399-411.

He, Q., Cheng, P., He, Q. and Liu, Y., (2005). The COP9 signalosome regulates the Neurospora circadian clock by controlling the stability of the SCF FWD-1 complex. Genes Dev. $\underline{19}$, 1518-1531. 
Helmstaedt, K., Schwier, E.U., Christmann, M., Nahlik, K., Westermann M., Harting R., Grond, S., Busch S. and Braus G.H., (2011). Recruitment of the inhibitor Cand1 to the cullin substrate adaptor site mediates interaction to the neddylation site. Mol Biol Cell. 22, 153164.

Hemming, A., (1944). Penicillin. J Natl Med Assoc. 트, 37-42.

Henry, K.W., Wyce, A., Lo, W.S., Duggan, L.J., Emre, N.C., Kao, C.F., Pillus, L., Shilatifard, A., Osley, M.A. and Berger, S.L., (2003). Transcriptional activation via sequential histone H2B ubiquitylation and deubiquitylation, mediated by SAGA-associated Ubp8. Genes Dev. 17, 2648-2663.

Hernández-Rodríguez, Y., Hastings, S. and Momany, M., (2012). The septin AspB in Aspergillus nidulans forms bars and filaments and plays roles in growth emergence and conidiation. Eukaryot Cell. 11, 311-323.

Hershko, A. and Ciechanover, A., (1998). The ubiquitin system. Annu Rev Biochem. $\underline{67}$, doi: 10.1146/annurev.biochem.67.1.425.

Hetfeld, B.K., Helfrich, A., Kapelari, B., Scheel, H., Hofmann, K., Guterman, A., Glickman, M., Schade, R., Kloetzel, P.M. and Dubiel, W., (2005). The zinc finger of the CSN-associated deubiquitinating enzyme USP15 is essential to rescue the E3 ligase Rbx1. Curr Biol. 15 , 1217-1221.

Hibbett, D.S., Binder, M., Bischoff, J.F., Blackwell, M., Cannon, F., Eriksson, O.E., Huhndorf, S., James, T., Kirk, P.M., Lücking, R., Lutzoni, F., Lumbsch, H.T., Matheny, P.B., McLaughlin, D.J., Powell, M.J., Redhead, S., Schoch, C.L., Spatafora, J.W., Stalpers, J.A., Vilgalys, R., Aime, M.C., Aptroot, A., Bauer, R., Begerow, D., Benny, G.L., Castlebury, L.A., Crous, P.W., Dai, Y., Gams, W., Geiser, D.M., Griffith, G.W., Hosaka, K., Humber, R.A., Hyde, K.D., Ironside, J.E., Kõljalg U, Kurtzman, C.P., Larsson, K., Lichtwardt, R., Mia, J., Mozley-Standridge, S., Oberwinkler, F., Parmasto, E., Reeb, V., , Rogers, J.D., Roux, C., Ryvarden, L., Sampaio, J. P., Sugiyama, J., Thorn, R.G., Tibell, L., Untereiner, W.A., Walker, C., Wang, Z., Weir, A., Weiss, M., White, M.M., Winka, K., Yao, Y.J. and Zhang, N., (2007). A higher-level phylogenetic classification of the fungi. Mycol Res. 111, 509-547.

Hicke, L., (2001). Protein regulation by monoubiquitin. Nat Rev Mol Cell Biol. 2, 195-201.

Hicke, L. and Dunn, R., (2003). Regulation of membrane protein transport by ubiquitin and ubiquitin-binding proteins. Annu Rev Cell Dev Biol. 19, 141-172.

Hotton, S.K. and Callis, J., (2008). Regulation of cullin RING ligases. Annu Rev Plant Biol. $\underline{59}$, 467-489.

Hu, M., Li, P., Li, M., Li, W., Yao, T., Wu, J.W., Gu, W., Cohen, R.E. and Shi, Y., (2002). Crystal structure of a UBP-family deubiquitinating enzyme in isolation and in complex with ubiquitin aldehyde. Cell. 111, 1041-1054.

Hua, Z. and Vierstra, R.D., (2011). The cullin-RING ubiquitin-protein ligases. Annu Rev Plant Biol. 62, 299-334. 
Huang, C.H., Su, M.G., Kao, H.J., Jhong, J.H., Weng, S.L. and Lee, T.Y., (2016). UbiSite: incorporating two-layered machine learning method with substrate motifs to predict ubiquitin-conjugation site on lysines. BMC Syst Biol. 10, doi: 10.1186/s12918-015-0246-z.

Huang, X., Langelotz, C., Hetfeld-Pechoc, B.K., Schwenk, W. and Dubiel, W., (2009). The COP9 signalosome mediates $\beta$-Catenin degradation by deneddylation and blocks adenomatous polyposis coli destruction via USP15. J Mol Biol. $\underline{39}$, 691-702.

Hunter, T., (2007). The age of crosstalk: Phosphorylation, ubiquitination, and beyond. Mol. Cell. $\underline{28}, 730-738$.

Hutchins, A.P., Liu, S., Diez, D. and Miranda-Saavedra, D., (2013). The repertoires of ubiquitinating and deubiquitinating enzymes in eukaryotic genomes. Mol Biol Evol. $\underline{30}$, $1172-1187$.

Ikeda, F., (2016). Linear ubiquitination signals in adaptive immune responses. Immunol Rev. 266, 222-236.

Ikeda, F. and Dikic, I., (2008). Atypical ubiquitin chains: new molecular signals. "Protein modifications: Beyond the usual suspects" review series. EMBO Rep. $\underline{9}, 536-542$.

Inglis, D.O., Binkley, J., Skrzypek, M.S., Arnaud, M.B., Cerqueira, G.C., Shah, P., Wymore, F., Wortman, J.R. and Sherlock, G., (2013). Comprehensive annotation of secondary metabolite biosynthetic genes and gene clusters of Aspergillus nidulans, A. fumigatus, A. niger and A. oryzae. BMC Microbiol. 13, doi: 10.1186/1471-2180-13-91.

Inoue, H., Nojima, H. and Okayama, H., (1990). High efficiency transformation of Escherichia coli with plasmids. Gene. $\underline{96}, 23-28$.

Isono, E. and Nagel, M.K., (2014). Deubiquitylating enzymes and their emerging role in plant

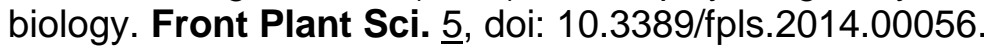

Ito, H., Fukuda, Y., Murata, K. and Kimura, A., (1983). Transformation of intact yeast cells treated with alkali cations. J Bacteriol. $\underline{153}, 163-168$.

Johnston, S.C., Riddle, S.M., Cohen, R.E. and Hill, C.P., (1999). Structural basis for the specificity of ubiquitin C- terminal hydrolases. EMBO J. 18, 3877-3887.

Jones, J., Wu, K., Yang, Y., Guerrero, C., Nillegoda, N., Pan, Z.Q. and Huang, L., (2009). A targeted proteomic analysis of the ubiquitin-like modifier Nedd8 and associated proteins. J Proteome Res. $\underline{7}, 1274-1287$.

Käfer, E., (1965). Origins of translocations in Aspergillus nidulans. Genetics. $\underline{52}, 217-232$.

Käfer, E., (1977). Meiotic and mitotic recombination in Aspergillus and its chromosomal aberrations. Adv Genet. 19, 33-131.

Kanehisa, M., Furumichi, M., Tanabe, M., Sato, Y. and Morishima, K., (2017). KEGG: New perspectives on genomes, pathways, diseases and drugs. Nucleic Acids Res. $\underline{45}$, D353D361. 
Karve, T.M. and Cheema, A.K., (2011). Small changes huge impact: the role of protein posttranslational modifications in cellular homeostasis and disease. J Amino Acids. 2011, doi: 10.4061/2011/207691.

Kato, N., Brooks, W. and Calvo, A.M., (2003). The expression of sterigmatocystin and penicillin genes in Aspergillus nidulans is controlled by veA, a gene required for sexual development. Am Soc Microbiol. 2, 1178-1186.

Kawakami, T., Chiba, T., Suzuki, T., Iwai, K., Yamanaka, K., Minato, N., Suzuki, H., Shimbara, N., Hidaka, Y., Osaka, F., Omata, M. and Tanaka, K., (2001). NEDD8 recruits E2-ubiquitin to SCF E3 ligase. EMBO J. 20, 4003-4012.

Kerscher, O., Felberbaum, R. and Hochstrasser, M., (2006). Modification of proteins by ubiquitin and ubiquitin-like proteins. Annu Rev Cell Dev Biol. 22, 159-180.

Kibbe, W.A., (2007). OligoCalc: an online oligonucleotide properties calculator. Nucleic Acids Res. 35 , W43-W46.

Kim, H., Han, K., Kim, K., Han, D., Jahng, K. and Chae, K., (2002). The veA gene activates sexual development in Aspergillus nidulans. Fungal Genet Biol. $\underline{37}, 72-80$.

Kim, J.H., Park, K.C., Chung, S.S., Bang, O. and Chung, C.H., (2003). Deubiquitinating enzymes as cellular regulators. J Biochem. 134, 9-18.

Kirisako, T., Kamei, K., Murata, S., Kato, M., Kanie, M., Sano, S., Tokunaga, F., Tanaka, K. and Iwai, K., (2006). A ubiquitin ligase complex assembles linear polyubiquitin chains. EMBO J. $\underline{25}, 4877-4887$.

Komander, D., Clague, M.J. and Urbé, S., (2009). Breaking the chains: structure and function of the deubiquitinases. Nat Rev Mol Cell Biol. $\underline{10}, 550-563$.

Kosugi, S., Hasebe, M., Tomita, M. and Yanagawa, H., (2009). Systematic identification of cell cycle-dependent yeast nucleocytoplasmic shuttling proteins by prediction of composite motifs. Proc Natl Acad Sci U S A. $\underline{106}, 10171-10176$.

Kouranti, I., McLean, J.R., Feoktistova, A., Liang, P., Johnson, A.E., Roberts-Galbraith, R.H. and Gould, K.L., (2010). A global census of fission yeast deubiquitinating enzyme localization and interaction networks reveals distinct compartmentalization profiles and overlapping functions in endocytosis and polarity. PLoS Biol. $\underline{8}$, doi: 10.1371/journal.pbio.1000471.

Kraft, E., Stone, S.L., Ma, L., Su, N., Gao, Y., Lau, O.S., Deng, X.W. and Callis, J., (2005). Genome analysis and functional characterization of the E2 and RING-type E3 ligase ubiquitination enzymes of Arabidopsis. Plant Physiol. 139, 1597-1611.

Krappmann, S., Bayram, Ö. and Braus, G.H., (2005). Deletion and allelic exchange of the Aspergillus fumigatus veA locus via a novel recyclable marker module. Eukaryot Cell. $\underline{4}$, 1298-1307.

Krijgsheld, P., Bleichrodt, R., van Veluw, G.J., Wang, F., Müller, W.H. Dijksterhuis, J. and Wösten, H.A., (2011). Development in Aspergillus. Stud Mycol. 74, doi: 10.3114/sim0006. 
Kück, U. and Hoff, B., (2006). Application of the nourseothricin acetyltransferase gene (nat1) as dominant marker for the transformation of filamentous fungi. Fungal Genet Newsl. $\underline{7}, 9-11$.

Laemmli, U.K., (1970). Cleavage of structural proteins during the assembly of the head of bacteriophage T4. Nature. $\underline{227}, 680-685$.

Lam, Y.A., Xu, W., DeMartino, G. and Cohen, R.E., (1997). Editing of ubiquitin conjugates by an isopeptidase in the $26 \mathrm{~S}$ proteasome. Nature. $\underline{385}, 737-740$.

Latgé, J.P., (1999). Aspergillus fumigatus and aspergillosis. Clin Microbiol Rev. 12, 310-350.

Lau, H.T., Suh, H.W., Golkowski, M. and Ong, S.E., (2014). Comparing SILAC- and stable isotope dimethyl-labeling approaches for quantitative proteomics. J Proteome Res. $\underline{13}$, 4164-4174.

Lauwers, E., Jacob, C. and André, B., (2009). K63-linked ubiquitin chains as a specific signal for protein sorting into the multivesicular body pathway. J Cell Biol. $\underline{185}$, 493-502.

Leach, M.D. and Brown, A.J., (2012). Posttranslational modifications of proteins in the pathobiology of medically relevant fungi. Eukaryot Cell. 11, 98-108.

Lecker, S.H., Goldberg, A.L. and Mitch, W.E., (2006). Protein degradation by the ubiquitinproteasome pathway in normal and disease states. J Am Soc Nephrol. 17, 1807-1819.

Lee, B.H., Lu, Y., Prado, M.A., Shi, Y., Tian, G., Sun, S., Elsasser, S., Gygi, S.P., King, R.W. and Finley, D., (2016). USP14 deubiquitinates proteasome-bound substrates that are ubiquitinated at multiple sites. Nature. $\underline{532}, 398-401$.

Lee, P.Y., Costumbrado, J., Hsu, C.Y. and Kim, Y.H., (2012). Agarose gel electrophoresis for the separation of DNA fragments. J Vis Exp. 므, doi: 10.3791/3923.

Li, M., Chen, D., Shiloh, A. and Luo, J., Nikolaev A.Y., Qin, Q. and Gu, W., (2002). Deubiquitination of p53 by HAUSP is an important pathway for p53 stabilization. Nature. $\underline{416}, 648-653$.

Li, S.J. and Hochstrasser, M., (2000). The yeast ULP2 (SMT4) gene encodes a novel protease specific for the ubiquitin-like Smt3 protein. Mol Cell Biol. 20, 2367-2377.

Li, W., Bengtson, M.H., Ulbrich, A., Matsuda, A., Reddy, V.A., Orth, A., Chanda, S.K. and Batalov, S. and Joazeiro, C.A.P., (2008). Genome-wide and functional annotation of human E3 ubiquitin ligases identifies MULAN, a mitochondrial E3 that regulates the organelle's dynamics and signaling. PLoS One. $\underline{3}$, doi: 10.1371/journal.pone.0001487.

Li, W. and Ye, Y., (2008). Polyubiquitin chains: functions, structures, and mechanisms. Cell Mol Life Sci. $\underline{65}$, 2397-2406.

Linden, H. and Macino, G., (1997). White collar 2, a partner in blue-light signal transduction, controlling expression of light-regulated genes in Neurospora crassa. EMBO J. $\underline{16}, 98-109$. 
Lindsey, R., Cowden, S., Hernández-Rodríguez, Y. and Momany, M., (2010). Septins AspA and AspC are important for normal development and limit the emergence of new growth foci in the multicellular fungus Aspergillus nidulans. Eukaryot Cell. $\underline{9}, 155-163$.

Lingaraju, G.M., Bunker, R.D., Cavadini, S., Hess, D., Hassiepen, U., Renatus, M., Fischer, E.S. and Thomä, N.H., (2014). Crystal structure of the human COP9 signalosome. Nature. $\underline{512}$, $161-165$.

Lockington, R.A. and Kelly, J.M., (2001). Carbon catabolite repression in Aspergillus nidulans. Eur J Biochem. 므, 1311-1321.

Lockington, R.A. and Kelly, J.M., (2002). The WD40-repeat protein CreC interacts with and stabilizes the deubiquitinating enzyme CreB in vivo in Aspergillus nidulans. Mol Microbiol. $\underline{43}, 1173-1182$.

Lyapina, S., Cope, G., Shevchenko, A., Serino, G., Tsuge, T., Zhou, C., Wolf, D. A., Wei, N., Shevchenko, A. and Deshaies, R.J., (2001). Promotion of NEDD-CUL1 conjugate cleavage by COP9 signalosome. Science. 292, 1382-1385.

Lykke-Andersen, K., Schaefer, L., Menon, S., Deng, X.W., Miller, J.B. and Wei, N., (2003). Disruption of the COP9 signalosome Csn2 subunit in mice causes deficient cell proliferation, accumulation of p53 and cyclin E, and early embryonic death. Mol Cell Biol. $\underline{23}, 6790-6797$.

Mabey Gilsenan, J., Cooley, J. and Bowyer, P., (2012). CADRE: The Central Aspergillus Data REpository 2012. Nucleic Acids Res. 40, D660-D666.

Machida, Y.J., Machida, Y., Vashisht, A.A., Wohlschlegel, J.A. and Dutta, A., (2009). The deubiquitinating enzyme BAP1 regulates cell growth via interaction with HCF-1. J Biol Chem. 284, 34179-34188.

Manian, S., Sreenivasaprasad, S. and Mills, P.R., (2001). DNA extraction method for PCR in mycorrhizal fungi. Lett Appl Microbiol. $\underline{33}$, 307-310.

Marchler-Bauer, A., Derbyshire, M.K., Gonzales, N.R., Lu, S., Chitsaz, F., Geer, L.Y., Geer, R.C., He, J., Gwadz, M., Hurwitz, D.I., Lanczycki, C.J., Lu, F., Marchler, G.H., Song, J.S., Thanki, N., Wang, Z., Yamashita, R.A., Zhang, D., Zheng, C. and Bryant, S.H., (2015). CDD: NCBl's conserved domain database. Nucleic Acids Res. 43, D222-D226.

Markina-Inarrairaegui, A., Etxebeste, O., Herrero-Garcia, E., Araujo-Bazan, L., FernandezMartinez, J., Flores, J.A., Osmani, S.A. and Espeso, E.A., (2011). Nuclear transporters in a multinucleated organism: functional and localization analyses in Aspergillus nidulans. Mol Biol Cell. 22, 3874-3886.

Mayorga, M.E. and Timberlake, W.E., (1990). Isolation and molecular characterisation of the Aspergillus nidulans $w A$ gene. Genetics. 126, 73-79.

Maytal-Kivity, V., Piran, R., Pick, E., Hofmann, K. and Glickman, M.H., (2002). COP9 signalosome components play a role in the mating pheromone response of $S$. cerevisiae. EMBO Rep. $\underline{3}, 1215-1221$. 
Mazzucotelli, E., Belloni, S., Marone, D., De Leonardis, A., Guerra, D., Di Fonzo, N., Cattivelli, L. and Mastrangelo, A., (2006). The E3 ubiquitin ligase gene family inplants: Regulation by degradation. Curr Genomics. $\underline{7}, 509-522$.

Meister, C., Gulko, M.K., Köhler, A.M. and Braus, G.H., (2016). The devil is in the details: comparison between COP9 signalosome (CSN) and the LID of the $26 \mathrm{~S}$ proteasome. Curr Genet. $\underline{62}, 129-136$.

Mergner, J., Heinzlmeir, S., Kuster, B. and Schwechheimer, C., (2015). Deneddylase1 deconjugates NEDD8 from non-cullin protein substrates in Arabidopsis thaliana. Plant Cell. 27, 741-753.

Metzger, M.B., Hristova, V.A. and Weissman, A.M., (2010). HECT and RING finger families of E3 ubiquitin ligases at a glance. J Cell Sci. $\underline{125}, 531-537$.

Mevissen, T.E.T., Hospenthal, M.K., Geurink, P.P., Elliott, P.R., Akutsu, M., Arnaudo, N., Ekkebus, R., Kulathu, Y., Wauer, T., El Oualid, F., Freund, S.M., Ovaa, H. and Komander, D., (2013). OTU deubiquitinases reveal mechanisms of linkage specificity and enable ubiquitin chain restriction analysis. Cell. 154, 169-184.

Miller, H.I., Henzel, W.J., Ridgway, J.B., Kuang, W.J., Chisholm, V. and Liu, C.C., (1989). Cloning and expression of a yeast ubiquitin-protein cleaving activity in Escherichia coli. Nat Biotechnol. $\underline{7}, 698-704$.

Mims, C.W., Richardson, E.A. and Timberlake, W.E., (1988). Ultrastructural analysis of conidiophore development in the fungus Aspergillus nidulans using freeze-substitution. Protoplasma. 144, 132-141.

Mohapatra, B., Ahmad, G., Nadeau, S., Zutshi, N., An, W., Scheffe, S., Dong, L., Feng, D., Goetz, B., Arya, P., Bailey, T.A., Palermo, N., Borgstahl, G.E., Natarajan, A., Raja, S.M., Naramura, M., Band, V. and Band, H., (2008). Protein tyrosine kinase regulation by ubiquitination: Critical roles of $\mathrm{Cbl}$ family ubiquitin ligases. Biochim Biophys Acta. $\underline{29}$, 1883-1889.

Momany, M., Zhao, J., Lindsey, R. and Westfall, P.J., (2001). Characterization of the Aspergillus nidulans septin (asp) gene family. Genetics. 157, 969-977.

Mooney, J.L. and Yager, L.N., (1990). Light is required for conidiation in Aspergillus nidulans. Genes Dev. 4, 1473-1482.

Morimoto, M., Nishida, T., Honda, R. and Yasuda, H., (2000). Modification of cullin-1 by ubiquitin-like protein Nedd8 enhances the activity of SCF(skp2) toward p27(kip1). Biochem Biophys Res Commun. 270, 1093-1096.

Mosadeghi, R., Reichermeier, K.M., Winkler, M., Schreiber, A., Reitsma, J.M., Zhang, Y., Stengel, F., Cao, J., Kim, M., Sweredoski, M.J., Hess, S., Leitner, A., Aebersold, R., Peter, M., Deshaies, R.J. and Enchev, R.I., (2016). Structural and kinetic analysis of the COP9 signalosome activation and the cullin-RING ubiquitin ligase deneddylation cycle. eLife. $\underline{5}$, doi: 10.7554/eLife.12102. 
Mukai, A., Yamamoto-Hino, M., Awano, W., Watanabe, W., Komada, M. and Goto, S., (2010). Balanced ubiquitylation and deubiquitylation of Frizzled regulate cellular responsiveness to Wg/Wnt. EMBO J. $\underline{29}, 2114-2125$.

Mukhopadhyay, D. and Dasso, M., (2007). Modification in reverse: the SUMO proteases. Trends Biochem Sci. 32, 286-295.

Müller, M.M., (2017). Post-translational modifications of protein backbones: Unique functions, mechanisms, and challenges. Biochemistry. $\underline{57}, 177-185$.

Mundt, K.E., Liu, C. and Carr, A.M., (2002). Deletion mutants in COP9 signalosome subunits in fission yeast Schizosaccharomyces pombe display distinct phenotypes. Mol Biol Cell. $\underline{13}$, 493-502.

Nahlik, K., Dumkow, M., Bayram, Ö., Helmstaedt, K., Busch, S., Valerius, O., Gerke, J., Hoppert, M., Schwier, E., Opitz, L., Westermann, M., Grond, S., Feussner, K., Goebel, C., Kaever, A., Meinicke, P., Feussner, I. and Braus, G.H., (2010). The COP9 signalosome mediates transcriptional and metabolic response to hormones, oxidative stress protection and cell wall rearrangement during fungal development. Mol Microbiol. $\underline{78}$, 964-979.

Nandi, D., Tahiliani, P., Kumar, A. and Chandu, D., (2006). The ubiquitin-proteasome system. J Biosc. 31, 137-155.

Naumann, M., Bech-Otschir, D., Huang, X., Ferrell, K. and Dubiel, W., (1999). COP9 signalosome-directed C-Jun activation/stabilisation is independent of JNK. J Biol Chem. 50, 35297-35300.

Nayak, T., Szewczyk, E., Oakley, C.E., Osmani, A., Ukil, L., Murray, S.L., Hynes, M.J., Osmani, S.A. and Oakley, B.R., (2006). A versatile and efficient gene-targeting system for Aspergillus nidulans. Genetics. $\underline{172}$, 1557-1566.

Nguyen, L.K., Kolch, W. and Kholodenko, B.N., (2013). When ubiquitination meets phosphorylation: a systems biology perspective of EGFR/MAPK signalling. Cell Commun Signal. 11, doi: 10.1186/1478-811X-11-52.

$\mathrm{Ni}, \mathrm{M}$. and Yu, J.H., (2007). A novel regulator couples sporogenesis and trehalose biogenesis in Aspergillus nidulans. PLoS One. 2. doi: 10.1371/journal.pone.0000970.

Nicassio, F., Corrado, N., Vissers, J.H., Areces, L.B., Bergink, S., Marteijn, J.A., Geverts, B., Houtsmuller, A.B., Vermeulen, W., di Fiore P.P. and Citterio, E. (2007). Report human USP3 is a chromatin modifier required for S Phase progression and genome stability. Curr Biol. 17, 1972-1977.

Nijman, S.M., Luna-Vargas, M.P., Velds, A., Brummelkamp, T.R., Dirac, A.M., Sixma, T.K. and Bernards, R., (2005). A genomic and functional inventory of deubiquitinating enzymes. Cell. 123, 773-786.

Noventa-Jordão, M.A., do Nascimento, A.M., Goldman, M.H., Terenzi, H.F. and Goldman, G.H., (2000). Molecular characterization of ubiquitin genes from Aspergillus nidulans: mRNA expression on different stress and growth conditions. Biochim Biophys Acta-Mol Cell 
Res. $1490,237-244$.

Nwakanma, C. and Unachukwu, M., (2017). Molds. In: Bevilacqua, A., Rosaria Corbo, M., Sinigaglia, M. (Eds.), The Microbiological Quality of Food. Woodhead Publishing, pp. 133148.

Oeckinghaus, A. and Ghosh, S., (2009). The NF-kappaB family of transcription factors and its regulation. Cold Spring Harb. Perspect. Biol. 1, doi: 10.1101/cshperspect.a000034.

Ohki, Y., Funatsu, N., Konishi, N. and Chiba, T., (2009). The mechanism of poly-NEDD8 chain formation in vitro. Biochem Biophys Res Commun. 381, 443-447.

Ohtake, F. and Tsuchiya, H., (2017). The emerging complexity of ubiquitin architecture. J Biochem. 161, 125-133.

Olma, M.H., Roy, M., Le Bihan, T., Sumara, I., Maerki, S., Larsen, B., Quadroni, M., Peter, M., Tyers, M. and Pintard, L., (2009). An interaction network of the mammalian COP9 signalosome identifies Dda1 as a core subunit of multiple Cul4-based E3 ligases. J Cell Sci. $122,1035-1044$.

Ong, S., Blagoev, B., Kratchmarova, I., Kristensen, D.B., Steen, H., Pandey, A. and Mann, M., (2002). Stable isotope labeling by amino acids in cell culture, SILAC, as a simple and accurate approach to expression proteomics. Mol Cell Proteomics. 1, 376-386.

Ong, S., Kratchmarova, I. and Mann, M., (2003). Properties of 13C-substituted arginine in stable isotope labeling by amino acids in cell culture (SILAC). J Proteome Res. 2, 173-181.

Oren-Giladi, P., Krieger, O., Edgar, B.A., Chamovitz, D.A. and Segal, D., (2008). Cop9 signalosome subunit 8 (CSN8) is essential for Drosophila development. Genes to Cells. $\underline{13}, 221-231$.

Osmani, A.H., May, G.S. and Osmani, S.A., (1999). The extremely conserved pyroA gene of Aspergillus nidulans is required for pyridoxine synthesis and is required indirectly for resistance to photosensitizers. J Biol Chem. 274, 23565-23569.

Osmani, S.A. and Mirabito, P.M., (2004). The early impact of genetics on our understanding of cell cycle regulation in Aspergillus nidulans. Fungal Genet Biol. 41, 401-410.

Özkaynak, E., Finley, D. and Solomon, M.J., (1987). The yeast ubiquitin genes: a family of natural gene fusions. EMBO J. $\underline{6}, 1429-1439$.

Palmer, J.M., Theisen, J.M., Duran, R.M., Grayburn, W.S., Calvo, A.M. and Keller, N.P., (2013). Secondary metabolism and development is mediated by LImF control of VeA subcellular localization in Aspergillus nidulans. PLoS Genet. ㅁ, doi: 10.1371/journal.pgen.1003193.

Papa, F.R., Amerik, A.Y. and Hochstrasser, M., (1999). Interaction of the Doa4 deubiquitinating enzyme with the yeast 26S proteasome. Mol Biol Cell. $\underline{10}, 741-756$.

Park, H.S., Ni, M., Cheol, K., Kim, Y.H. and Yu, J.H., (2012). The role, interaction and regulation of the velvet regulator VelB in Aspergillus nidulans. PLoS One. $\underline{7}$, doi: 
10.1371/journal.pone.0045935.

Park, K.C., Choi, E.J., Min, S.W., Chung, S.S., Kim, H., Suzuki, T., Tanaka, K. and Chung, C.H., (2000). Tissue-specificity, functional characterization and subcellular localization of a rat ubiquitin-specific processing protease, UBP109, whose mRNA expression is developmentally regulated. Biochem J 349, 443-453.

Pathare, G.R., Nagy, I., Sledz, P., Anderson, D.J., Zhou, H.J., Pardon, E., Steyaert, J., Forster, F., Bracher, A. and Baumeister, W., (2014). Crystal structure of the proteasomal deubiquitylation module Rpn8-Rpn11. Proc Natl Acad Sci U S A. 111, 2984-2989.

Paul, S., (2008). Dysfunction of the ubiquitin-proteasome system in multiple disease conditions: Therapeutic approaches. BioEssays. $\underline{30}, 1172-1184$.

Pees, E., (1967). Genetic fine structure and polarized negative interference at the lys-51 (FL) locus of Aspergillus nidulans. Genetica. $\underline{38}$, 275-304.

Pereira, R.V., de S Gomes, M., Olmo, R.P., Souza, D.M., Cabral, F.J., Jannotti-Passos, L.K., Baba, E.H., Andreolli, A.B., Rodrigues, V., Castro-Borges, W. and Guerra-Sá, R., (2015). Ubiquitin-specific proteases are differentially expressed throughout the Schistosoma mansoni life cycle. Parasit. Vectors. $\underline{8}$, doi.org/10.1186/s13071-015-0957-4.

Petroski, M.D. and Deshaies, R.J., (2005). Function and regulation of cullin-RING ubiquitin ligases. Nat Rev Mol Cell Biol. $\underline{6}$, 9-20.

Pick, E. and Pintard, L., (2009). In the land of the rising sun with the COP9 signalosome and related Zomes. EMBO Rep. 10, 343-348.

Pickart, C.M. and Eddins, M.J., (2004). Ubiquitin: structures, functions, mechanisms. Biochim Biophys Acta. $1695,55-72$.

Pöggeler, S., Nowrousian, M. and Kück, U., (2018). The Mycota I. In: Anke, T., Schüffler, A. (Eds.), Fruiting-body development in ascomycetes. Springer International Publishing AG, pp. 1-56.

Prabakaran, S., Lippens, G., Steen, H. and Gunawardena, J., (2012). Post-translational modification: nature's escape from genetic imprisonment and the basis for dynamic information encoding. Wiley Interdiscip Rev Syst Biol Med. 4, 565-583.

Punt, P.J., Oliver, R.P., Dingemanse, M.A., Pouwels, P.H. and van den Hondel, C.A., (1987). Transformation of Aspergillus based on the hygromycin B resistance marker from Escherichia coli. Gene. 56, 117-124.

Purschwitz, J., Müller, S., Kastner, C., Schöser, M., Haas, H., Espeso, E.A., Atoui, A., Calvo, A.M. and Fischer, R., (2008). Functional and physical interaction of blue- and red-light sensors in Aspergillus nidulans. Curr Biol. 18, 255-259.

Rabilloud, T. and Lelong, C., (2011). Two-dimensional gel electrophoresis in proteomics: a tutorial. J Proteomics. $\underline{74}$, 1829-1841. 
Radivojac, P., Vacic, V., Haynes, C., Cocklin, R.R., Mohan, A., Heyen, J.W., Goebl, M.G. and lakoucheva, L.M., (2011). Identification, analysis and prediction of protein ubiquitination sites. Proteins. $\underline{78}, 365-380$.

Rappsilber, J., Ishihama, Y. and Mann, M., (2003). Stop and go extraction tips for matrixassisted laser desorption/ionization, nanoelectrospray, and LC/MS sample pretreatment in proteomics. Anal Chem. 포, 663-670.

Rauniyar, N. and Yates, J.R., (2014). Isobaric labeling-based relative quantification in shotgun proteomics. J Proteome Res. 13, 5293-5309.

Rauscher, S., Pacher, S., Hedtke, M., Kniemeyer, O. and Fischer, R., (2016). A phosphorylation code of the Aspergillus nidulans global regulator VelvetA (VeA) determines specific functions. Mol Microbiol. 99, 909-924.

Ravid, T. and Hochstrasser, M., (2008). Diversity of degradation signals in the ubiquitinproteasome system. Nat Rev Mol Cell Biol. $\underline{9}$, 679-690.

Rechsteiner, M. and Rogers, S.W., (1996). PEST sequences and regulation by proteolysis. Trends Biochem Sci. 21, 267-271.

Regulin, A. and Kempken, F., (2018). Fungal genotype determines survival of Drosophila melanogaster when competing with Aspergillus nidulans. PLoS One. 13, doi: 10.1371/journal.pone.0190543.

Renatus, M., Parrado, S.G., Arcy, A.D., Eidhoff, U., Gerhartz, B., Hassiepen, U., Pierrat, B., Riedl, R., Vinzenz, D., Worpenberg, S. and Kroemer, M., (2006). Structural basis of ubiquitin recognition by the deubiquitinating protease USP2. Structure. 14, 1293-1302.

Reyes-Turcu, F.E., Horton, J.R., Mullally, J.E., Heroux, A., Cheng, X. and Wilkinson, K.D., (2006). The ubiquitin binding domain $\mathrm{ZnF}$ UBP recognizes the C-terminal diglycine motif of unanchored ubiquitin. Cell. 124, 1197-1208.

Reyes-Turcu, F.E., Ventii, K.H. and Wilkinson, K.D., (2009). Regulation and cellular roles of ubiquitin-specific deubiquitinating enzymes. Annu Rev Biochem. 78, 363-397.

Rhee, S.G., Ho, Z.C. and Kim, K., (2005). Peroxiredoxins: A historical overview and speculative preview of novel mechanisms and emerging concepts in cell signaling. Free Radic Biol Med. $38,1543-1552$.

Rittinger, K. and Ikeda, F., (2017). Linear ubiquitin chains: enzymes, mechanisms and biology. Open Biol. $\underline{7}$, doi: 10.1098/rsob.170026.

Robinson, P.K., (2015). Enzymes: principles and biotechnological applications. Essays Biochem. 59, doi: 10.1042/bse0590001.

Robzyk, K., Recht, J. and Osley, M.A., (2000). Rad6-dependent ubiquitination of histone H2B in yeast. Science. $\underline{287}, 501-504$.

Rodrigues, A.J., Coppola, G., Santos, C., Costa Mdo. C., Ailion, M., Sequeiros, J., Geschwind, 
D.H. and Maciel, P., (2007). Functional genomics and biochemical characterization of the C. elegans orthologue of the Machado-Joseph disease protein ataxin-3. FASEB J. 21, $1126-1136$.

Rodriguez, R.J., White, J.F. Jr., Arnold, A.E. and Redman, R.S., (2009). Fungal endophytes: diversity and functional roles. New Phytol. $\underline{2}, 314-330$.

Rogers, S., Wells, R. and Rechsteiner, M., (1986). Amino acid sequences common to rapidly degraded proteins: the PEST hypothesis. Science. 234, 364-368.

Romero-Calvo, I., Ocón, B., Martínez-Moya, P., Suárez, M.D., Zarzuelo, A., Martínez-Augustin, O. and de Medina, F.S., (2010). Reversible Ponceau staining as a loading control alternative to actin in western blots. Anal Biochem. 401, 318-320.

Ruger-Herreros, C., Rodríguez-Romero, J., Fernández-Barranco, R., Olmedo, M., Fischer, R., Corrochano, L.M. and Canovas, D., (2011). Regulation of conidiation by light in Aspergillus nidulans. Genetics. 188, 809-822.

Ryan, B.J., Nissim, A. and Winyard, P.G., (2014). Redox biology oxidative post-translational modifications and their involvement in the pathogenesis of autoimmune diseases. Redox Biol. 2ㅇ, 715-724.

Sadowski, M., Suryadinata, R., Tan, A.R., Nur, S., Roesley, A. and Sarcevic, B., (2012). Protein monoubiquitination and polyubiquitination generate structural diversity to control distinct biological processes. IUBMB Life. $\underline{64}, 136-142$.

Saeki, Y., Isono, E., Oguchi, T., Shimada, M., Sone, T., Kawahara, H., Yokosawa, H. and Toh-e, A., (2004). Intracellulary inducible, ubiquitin hydrolase-insensitive tandem ubiquitins inhibit the $26 S$ proteasome activity and cell division. Genes Genet. Syst. 79, 77-86.

Saiki, R., Gelfand, D., Stoffel, S., Scharf, S., Higuchi, R., Horn, G., Mullis, K. and Erlich, H., (1988). Primer-directed enzymatic amplification of DNA with a thermostable DNA polymerase. Science. $\underline{239}$, 487-491.

Samson, R.A., Visagie, C.M., Houbraken, J., Hubka, V., Perrone, G., Seifert, K.A., Susca, A., Tanney, J.B., Varga, J., Kocsub, S., Szigeti, G., Yaguchi, T. and Frisvad, J.C., (2014). Phylogeny, identification and nomenclature of the genus Aspergillus. Stud. Mycol. $\underline{78}$, 141-173.

Sanchez, J.F., Chiang, Y.-M., Szewczyk, E., Davidson, A.D., Ahuja, M., Elizabeth Oakley, C., Woo Bok, J., Keller, N., Oakley, B.R. and Wang, C.C.C., (2010). Molecular genetic analysis of the orsellinic acid/F9775 genecluster of Aspergillus nidulans. Mol BioSyst. $\underline{6}$, 587-593.

Sanchez, J.F. and Wang, C.C.C., (2013). The chemical identification and analysis of Aspergillus nidulans secondary metabolites. Methods Mol Biol. 944, 97-109.

Santos, A.L. and Lindner, A.B., (2017). Protein posttranslational modifications: roles in aging and age-related disease. Oxid Med Cell Longev. 2017, doi: 10.1155/2017/5716409. 
Sarikas, A., Hartmann, T. and Pan, Z., (2011). The cullin protein family. Genome Biol. 12, doi: 10.1186/gb-2011-12-4-220.

Sarikaya-Bayram, Ö., Bayram, Ö., Feussner, K., Kim, J.H., Kim, H.S., Kaever, A., Feussner, I., Chae, K.S., Han, D.M., Han, K.H. and Braus, G.H., (2014). Membrane-bound methyltransferase complex VapA-VipC-VapB guides epigenetic control of fungal development. Dev Cell. 29, 406-420.

Sarikaya-Bayram, Ö., Bayram, Ö., Valerius, O., Park, H.S., Irniger, S., Gerke, J., Ni, M., Han, K.H., Yu, J.H. and Braus, G.H., (2010). LaeA control of velvet family regulatory proteins for light-dependent development and fungal cell-type specificity. PLoS Genet. $\underline{6}$, doi: 10.1371/journal.pgen.1001226.

Sarikaya-Bayram, Ö., Palmer, J.M., Keller, N., Braus, G.H. and Bayram, Ö., (2015). One Juliet and four Romeos: VeA and its methyltransferases. Front Microbiol. $\underline{6}$, doi: 10.3389/fmicb.2015.00001.

Sato, Y., Goto, E., Shibata, Y., Kubota, Y., Yamagata, A., Goto-Ito, S., Kubota, K., Inoue, J., Takekawa, M., Tokunaga, F. and Fukai, S., (2015). Structures of CYLD USP with Met1-or Lys63-linked diubiquitin reveal mechanisms for dual specificity. Nat Struct Mol Biol. 22 , 222-229.

Schaefer, J.B. and Morgan, D.O., (2011). Protein-linked ubiquitin chain structure restricts activity of deubiquitinating enzymes. J Biol Chem. 286, 45186-45196.

Scheel, H. and Hofmann, K., (2005). Prediction of a common structural scaffold for proteasome lid, COP9 signalosome and elF3 complexes. BMC Bioinformatics. $\underline{6}$, doi: 10.1186/14712105-6-71.

Schmitt, K., Smolinski, N., Neumann, P., Schmaul, S., Hofer-Pretz, V. and Braus, G.H., (2017). Asc1p/RACK1 connects ribosomes to eukaryotic phosphosignaling. Mol Cell Biol. $\underline{37}$, doi: 10.1128/MCB.00279-16.

Schmittgen, T.D. and Livak, K.J., (2008). Analyzing real-time PCR data by the comparative CT method. Nat Protoc. $\underline{3}, 1101-1108$.

Schwämmle, V., Sidoli, S., Ruminowicz, C., Wu, X., Lee, C., Helin, K. and Jensen, O.N., (2016). Systems level analysis of histone $\mathrm{H} 3$ post-translational modifications (PTMs) reveals features of PTM crosstalk in chromatin regulation. Mol Cell Proteomics. 21, 2715-2729.

Schweitzer, K., Bozko, P.M., Dubiel, W. and Naumann, M., (2007). CSN controls NF-kB by deubiquitinylation of ІкBa. EMBO J. 266, 1532-1541.

Seeger, M., Kraft, R., Ferrell, K., Schade, D., Gordon, C., Naumann, M. and Dubiel, W., (1998). A novel protein complex involved in signal transduction possessing similarities to $26 \mathrm{~S}$ proteasome subunits. FASEB J. $12,469-478$.

Sharon, M., Mao, H., Boeri Erba, E., Stephens, E., Zheng, N. and Robinson, C.V., (2009). Symmetrical modularity of the COP9 signalosome complex suggests its multifunctionality. Structure. 17, 31-40. 
Shevchenko, A., Tomas, H., Havliš, J., Olsen, J.V. and Mann, M., (2007). In-gel digestion for mass spectrometric characterization of proteins and proteomes. Nat Protoc. 1 , 2856-2860.

Sievers, F., Wilm, A., Dineen, D., Gibson, T.J., Karplus, K., Li, W., Lopez, R., McWilliam, H., Remmert, M., Söding, J., Thompson, J.D. and Higgins, D.G., (2011). Fast, scalable generation of high-quality protein multiple sequence alignments using Clustal Omega. Mol Syst Biol. ㄱ, doi: 10.1038/msb.2011.75.

Simpson, R.J., (2006). Fragmentation of proteins using trypsin. CSH Protoc. $\underline{5}$, doi: $10.1101 /$ pdb.prot. 4550 .

Soboleva, T.A., Jans, D.A., Johnson-Saliba, M. and Baker, R.T., (2005). Nuclear-cytoplasmic shuttling of the oncogenic mouse UNP/USP4 deubiquitylating enzyme. J Biol Chem. 280, 745-752.

Sohn, K.T. and Yoon, K.S., (2002). Ultrastructural study on the cleistothecium development in Aspergillus nidulans. Mycobiology. 30, 117-127.

Song, E.J., Werner, S.L., Neubauer, J. and Stegmeier, F., (2010). Deubiquitinating enzyme control reversible ubiquitination at the spliceosome. Genes Dev. 24, 1434-1447.

Southern, E.M., (1975). Detection of specific sequences among DNA fragments separated by gel electrophoresis. J Mol Biol. 98, 503-517.

Sowa, M.E., Bennett, E.J., Gygi, S.P. and Harper, J.W., (2009). Defining the human deubiquitinating enzyme interaction landscape. Cell. 138, 389-403.

Spasser, L. and Brik, A., (2012). Chemistry and biology of the ubiquitin signal. Angew Chemie $\underline{51}, 6840-6862$.

Spence, J., Sadis, S. and Haas, A.L., (1995). A ubiquitin mutant with specific defects in DNA repair and multiubiquitination. Mol Cell Biol. $\underline{15}$, 1265-1273.

Stajich, J.E., Harris, T., Brunk, B.P., Brestelli, J., Fischer, S., Harb, O.S., Kissinger, J.C., Li, W., Nayak, V., Pinney, D.F., Stoeckert, C.J. Jr. and Roos, D.S., (2012). FungiDB: an integrated functional genomics database for fungi. Nucleic Acids Res. $\underline{40}$, 675-681.

Stinnett, S.M., Espeso, E.A., Cobeño, L., Araújo-Bazán, L. and Calvo, A.M., (2007). Aspergillus nidulans $\mathrm{VeA}$ subcellular localization is dependent on the importin a carrier and on light. Mol Microbiol. $\underline{63}, 242-255$.

Sun, S.C., (2011). Non-canonical NF-kB signaling pathway. Cell Res. 211, 71-85.

Sun, Y., Wilson, M.P. and Majerus, P.W., (2002). Inositol 1,3,4-triphosphate 5/6-kinase associates with the COP9 signalosome by binding to CSN1. J Biol Chem. 277, 4575945764.

Sun, Z. and Andersson, R., (2002). NF-kB activation and inhibition: A review. SHOCK 18 , 99106. 
Swaminathan, S., Amerik, A.Y. and Hochstrasser, M., (1999). The Doa4 deubiquitinating enzyme is required for ubiquitin homeostasis in yeast. Mol Biol Cell $\underline{10}$, 2583-2594.

Szewczyk, E., Nayak, T., Oakley, C.E., Edgerton, H., Xiong, Y., Taheri-Talesh, N., Osmani, S.A. and Oakley, B.R., (2006). Fusion pcr and gene targeting in Aspergillus nidulans. Nat Protoc. 1, 3111-3120.

Tan, J.M., Wong, E.S., Kirkpatrick, D.S., Pletnikova, O., Ko, H.S., Tay, S.P., Ho, M.W., Troncoso, J., Gygi, S.P., Lee, M.K., Dawson, V.L., Dawson, T.M. and Lim, K.L., (2018). Lysine 63-linked ubiquitination promotes the formation and autophagic clearance of protein inclusions associated with neurodegenerative diseases. Hum Mol Genet. 17, 431-439.

Tanase, M., Urbanska, A.M., Zolla, V., Clement, C.C., Huang, L., Morozova, K., Follo, C., Goldberg, M., Roda, B., Reschiglian, P. and Santambrogio, L., (2016). Role of carbonyl modifications on aging-associated protein aggregation. Sci Rep. $\underline{6}$, doi: 10.1038/srep19311.

Tasaki, T., Sriram, S.M., Park, K.S. and Kwon, Y.T., (2012). The N-End rule pathway. Annu Rev Biochem. 81, 261-289.

Terrell, J., Shih, S., Dunn, R. and Hicke, L., (1998). A function for monoubiquitination in the internalization of a G protein-coupled receptor. Mol Cell. 1, 193-202.

Thieme, K.G., (2017). The Zinc cluster transcription factor ZtfA is an activator of asexual development and secondary metabolism and regulates the oxidative stress response in the filamentous fungus Aspergillus nidulans. Göttingen: Institute of Microbiology and Genetics, Georg-August University Göttingen.

Thieme, S., (2018). Insertion of an intrinsically disordered domain in VelB supports selective heterodimer formation of fungal velvet domain regulatory proteins in Aspergillus nidulans. Göttingen: Institute of Microbiology and Genetics, Georg-August University Göttingen.

Thrower, J.S., Hoffman, L., Rechsteiner, M. and Pickart, C.M., (2000). Recognition of the polyubiquitin proteolytic signal. EMBO J. 19, 94-102.

Timberlake, W.E., (1980). Developmental gene regulation in Aspergillus nidulans. Dev Biol. $\underline{78}$, 497-510.

Timberlake, W.E., (1990). Molecular genetics of Aspergillus development. Annu Rev Genet. $\underline{18}$, 5-36.

Tobias, J.W. and Varshavsky, A., (1991). Cloning and functional analysis of the ubiquitin-specific protease gene UBP1 of Saccharomyces cerevisiae. J Biol Chem. 266, 12021-12028.

Tomoda, K., Kubota, Y., Arata, Y., Mori, S., Maeda, M., Tanaka, T., Yoshida, M., Yoneda-Kato, N. and Kato, J.Y., (2002). The cytoplasmic shuttling and subsequent degradation of p27Kip1mediated by Jab1/CSN5 and the COP9 signalosome complex. J Biol Chem. 277, 2302-2310. 
Towbin, H., Staehelin, T. and Gordon, J., (1979). Electrophoretic transfer of proteins from polyacrylamide gels to nitrocellulose sheets: procedure and some applications. Proc Natl Acad Sci U S A. $\underline{76}$, 4350-4354.

Trotman, L.C., Wang, X., Alimonti, A., Chen, Z., Teruya-Feldstein, J., Yang, H., Pavletich, N.P., Carver, B.S., Cordon-Cardo, C., Erdjument-Bromage, H., Tempst, P., Chi, S.G., Kim, H.J., Misteli, T., Jiang, X. and Pandolfi, P.P., (2007). Ubiquitination regulates PTEN nuclear import and tumor suppression. Cell. 128, 141-156.

Tsitsigiannis, D.I., Kowieski, T.M., Zarnowski, R. and Keller, N.P., (2005). Three putative oxylipin biosynthetic genes integrate sexual and asexual development in Aspergillus nidulans. Microbiology. 151, 1809-1821.

Tsitsigiannis, D.I., Zarnowski, R. and Keller, N.P., (2004). The lipid body protein, PpoA, coordinates sexual and asexual sporulation in Aspergillus nidulans. J Biol Chem. $\underline{279}$, 11344-11353.

Tsou, W., Sheedlo, M.J., Morrow, M.E., Blount, J.R., McGregor, K.M., Chittaranjan, D. and Todi, S.V., (2012). Systematic analysis of the physiological importance of deubiquitinating enzymes. PLoS One. $\underline{7}$, doi: 10.1371/journal.pone.0043112.

Tyanova, S., Temu, T., Sinitcyn, P., Carlson, A., Hein, M.Y., Geiger, T., Mann, M. and Cox, J., (2016). The Perseus computational platform for comprehensive analysis of (prote)omics data. Nat Methods. $\underline{13}, 731-740$.

Uhle, S., Medalia, O., Waldron, R., Dumdey, R., Henklein, P., Bech-Otschir, D., Huang, X., Berse, M., Sperling, J., Schade, Ė. and Dubiel, W., (2003). Protein kinase CK2 and protein kinase D are associated with the COP9 signalosome. EMBO J. 22, 1302-1312.

Ulrich, H.D., (2008). The fast-growing business of SUMO chains. Mol Cell. $\underline{32}, 301-305$.

Untergasser, A., Cutcutache, I., Koressaar, T., Ye, J., Faircloth, B.C., Remm, M. and Rozen, S.G., (2012). Primer3-new capabilities and interfaces. Nucleic Acids Res. 40, doi: $10.1093 /$ nar/gks596.

van der Horst, A., de Vries-Smits, A.M., Brenkman, A.B., van Triest, M.H., van den Broek, N., Colland, F., Maurice, M.M. and Burgering, B.M., (2006). FOXO4 transcriptional activity is regulated by monoubiquitination and USP7/HAUSP. Nat Cell Biol. $\underline{8}, 1064-1073$.

Ventii, K.H. and Wilkinson, K.D., (2009). Protein partners of deubiquitinating enzymes. Biochem J. 414, 161-175

Verma, R., Aravind, L., Oania, R., McDonald, W.H., Yates, J.R., Koonin, E.V. and Deshaies, R.J., (2002). Role of Rpn11 metalloprotease in deubiquitination and degradation by the $26 \mathrm{~S}$ proteasome. Science. $\underline{298}, 611-615$.

Vijay-Kumar, S., Bugg, C.E. and Cook, J., (1987). Structure of ubiquitin refined at $1.8 \AA$ resolution. J Mol Biol. $\underline{194}$, 531-544. 
Virag, A., Lee, M.P., Si, H. and Harris, S.D., (2007). Regulation of hyphal morphogenesis by cdc42 and rac1 homologues in Aspergillus nidulans. Mol Microbiol. 66, 1579-1596.

Vlasschaert, C., Xia, X., Coulombe, J. and Gray, D.A., (2015). Evolution of the highly networked deubiquitinating enzymes USP4, USP15, and USP11. BMC Evol Biol. 15, doi: 10.1186/s12862-015-0511-1.

von Zeska Kress, M.R., Harting, R., Bayram, Ö., Christmann, M., Irmer, H., Valerius, O., Schinke, J., Goldman, G.H. and Braus, G.H., (2012). The COP9 signalosome counteracts the accumulation of cullin SCF ubiquitin E3 RING ligases during fungal development. Mol Microbiol. $\underline{83}, 1162-1177$.

Wada, H., Kito, K., Caskey, L.S., Yeh, E.T. and Kamitani, T., (1998). Cleavage of the C-terminus of NEDD8 by UCH-L3. Biochem Biophys Res Commun. 251, 688-692.

Wada, H., Yeh, E.T. and Kamitani, T., (1999). Identification of NEDD8-conjugation site in human cullin-2. Biochem Biophys Res Commun. 257, 100-105.

Wang, F., Dijksterhuis, J., Wyatt, T., Wösten, H.A. and Bleichrodt, R.J., (2015). VeA of Aspergillus niger increases spore dispersing capacity by impacting conidiophore architecture. Antonie van Leeuwenhoek. 107, 187-199.

Wang, J. and Maldonado, M.A., (2006). The ubiquitin-proteasome system and its role in inflammatory and autoimmune diseases. Cell Mol Immunol. $\underline{3}, 255-261$.

Wang, Y. and Dasso, M., (2009). SUMOylation and deSUMOylation at a glance. J Cell Sci. 122 , 4249-4252.

Wee, S., Geyer, R.K., Toda, T. and Wolf, D.A., (2005). CSN facilitates cullin-RING ubiquitin ligase function by counteracting autocatalytic adapter instability. Nat Cell Biol. $\underline{7}$, 387-391.

Wei, N. and Chamovitz, D.A., (1994). Arabidopsis COP9 is a component of a novel signaling complex mediating light control of development. Cell. $\underline{78}, 117-124$.

Wei, N. and Deng, X.W., (2003). The COP9 signalosome. Annu Rev Cell Dev Biol. 19, 261286.

Wei, N., Serino, G. and Deng, X.W., (2008). The COP9 signalosome: more than a protease. Trends Biochem Sci. $\underline{33}$, 592-600.

Weidner, G., Steffan, B. and Brakhage, A.A., (1997). The Aspergillus nidulans lysF gene encodes homoaconitase, an enzyme involved in the fungus-specific lysine biosynthesis pathway. Mol Gen Genet. 255, 237-247.

West, M.H. and Bonner, W.M., (1980). Histone 2B can be modified by the attachment of ubiquitin. Nucleic Acids Res. $\underline{8}, 4671-4680$.

Wiborg, O., Pedersen, M.S., Wind, A., Berglund, L.E., Marcker, K.A. and Vuust, J., (1985). The human ubiquitin multigene family: some genes contain multiple directly repeated ubiquitin coding sequences. EMBO J. 4 , 755-759. 
Willems, A.R., Schwab, M. and Tyers, M., (2004). A hitchhiker's guide to the cullin ubiquitin ligases: SCF and its kin. Biochim Biophys Acta. 1695, 133-170.

Winborn, B.J., Travis, S.M., Todi, S. V., Scaglione, K.M., Xu, P., Williams, A.J., Cohen, R.E., Peng, J. and Paulson, H.L., (2008). The deubiquitinating enzyme ataxin-3, a polyglutamine disease protein, edits Lys63 linkages in mixed linkage ubiquitin chains. J Biol Chem. $\underline{283}$, 26436-26443.

Wolkow, T.D., Harris, S.D. and Hamer, J.E., (1996). Cytokinesis in Aspergillus nidulans is controlled by cell size, nuclear positioning and mitosis. J Cell Sci. 2188, 2179-2188.

Worden, E.J., Dong, K.C., Worden, E.J., Dong, K.C. and Martin, A., (2017). An AAA motordriven mechanical switch in Rpn11 controls deubiquitination at the $26 \mathrm{~S}$ proteasome article. Mol Cell. $\underline{67}, 799-811$.

Worden, E.J., Padovani, C. and Martin, A., (2014). Structure of the Rpn11 - Rpn8 dimer reveals mechanisms of substrate deubiquitination during proteasomal degradation. Nat Struct Mol Biol. 21, 220-227.

Wu, K., Chen, A., Tan, P. and Pan, Z.Q., (2002). The Nedd8-conjugated ROC1-CUL1 core ubiquitin ligase utilizes Need8 charged surface residues for efficient polyubiquitin chain assembly catalyzed by Cdc34. J Biol Chem. $\underline{277}$, 516-527.

Wu, K., Yamoah, K., Dolios, G., Gan-Erdene, T., Tan, P., Chen, A., Lee, C.G., Wei, N., Wilkinson, K.D., Wang, R. and Pan, Z.Q., (2003). DEN1 is a dual function protease capable of processing the C-terminus of Nedd8 and deconjugating hyperneddylated CUL1. J Biol Chem. 278, 28882-28891.

Xiang, X. and Plamann, M., (2003). Cytoskeleton and motor proteins in filamentous fungi. Curr Opin Microbiol. $\underline{6}, 628-633$.

Xu, P., Duong, D.M., Seyfried, N.T., Cheng, D., Xie, Y., Robert, J., Rush, J., Hochstrasser, M., Finley, D. and Peng, J., (2009). Quantitative proteomics reveals the function of unconventional ubiquitin chains in proteasomal degradation. Cell. 137, 133-145.

Yang, M., Chen, T., Li, X., Yu, Z., Tang, S., Wang, C., Gu, Y., Liu, Y., Xu, S., Li, W., Zhang, X., Wang, J. and Cao, X., (2015). K33-linked polyubiquitination of Zap70 by Nrdp1 controls CD8 + T cell activation. Nat Immunol. $\underline{16}, 1253-1262$.

Yang, W.L., Zhang, X. and Lin, H.K., (2010). Emerging role of Lys-63 ubiquitination in protein kinase and phosphatase activation and cancer development. Oncogene. $\underline{29}$, 4493-4503.

Yao, T. and Cohen, R.E., (2002). A cryptic protease couples deubiquitination and degradation by the proteasome. Nature. $\underline{419}, 403-407$.

Yao, T., Song, L., Xu, W., Demartino, G.N., Florens, L., Swanson, S.K., Washburn, M.P., Conaway, R.C., Conaway, J.W. and Cohen, R.E., (2006). Proteasome recruitment and activation of the Uch37 deubiquitinating enzyme by Adrm1. Nat Cell Biol. $\underline{8}, 994-1002$. 
Ye, Y. and Rape, M., (2011). Building ubiquitin chains: E2 enzymes at work. Nat Rev Mol Cell Biol. 10, 755-764.

Ye, Y., Scheel, H., Hofmann, K. and Komander, D., (2009). Dissection of USP catalytic domains reveals five common insertion points. Mol Biosyst. $\underline{5}$, 1797-1808.

Yu, H., Mashtalir, N., Daou, S., Hammond-Martel, I., Ross, J., Sui, G., Hart, G.W., Rauscher, F.J. 3rd, Drobetsky, E., Milot, E., Shi, Y. and Affar, E.B., (2010). The ubiquitin carboxyl hydrolase BAP1 forms a ternary complex with YY1 and HCF-1 and is a critical regulator of gene expression. Mol Cell Biol. $\underline{30}$, 5071-5085.

Yu, J., (2012). Current understanding on aflatoxin biosynthesis and future perspective in reducing aflatoxin contamination. Toxins (Basel). $\underline{4}$, 1024-1057.

Yu, J.H. and Keller, N., (2005). Regulation of secondary metabolism in filamentous fungi. Annu Rev Phytopathol. $\underline{43}$, 437-458.

Zhang, X.Y., Varthi, M., Sykes, S.M., Philips, C., Warzecha, C., Zhu, W., Wyce, A., Thorne, A.W., Berger, S.L. and McMahon, S.B., (2008). The putative cancer stem cell marker USP22 is a subunit of the human SAGA complex required for activator-driven transcription and cell cycle progression. Mol Cell. $\underline{29}, 102-111$.

Zhou, C., Wee, S., Rhee, E., Naumann, M., Dubiel, W. and Wolf, D.A., (2003). Fission yeast COP signalosome suppresses cullin activity through recruitment of the deubiquitylating enzyme Ubp12p. Mol Cell. 11, 927-938. 


\section{Supplementary Material}

Table S1: Mean SILAC ratios of proteins, which abundances are decreased in the presence of CsnE.

Logarithmized SILAC ratios (=fold changes (FC)) are depicted in the table. Fold changes of $\triangle \operatorname{csn} E$ compared to the L-lysine auxotrophic background strain $\Delta l y s A$ are shown in the very left column $(\Delta c s n E / \Delta / y s)$ The FCs are the mean of three independent biological replicates. The standard deviation (SD) is shown as well. Furthermore, the mean fold changes of proteins in $\triangle c s n E$ compared to its complementation strain $C s n E^{\text {comp }}$ with the corresponding standard deviations are shown. $\log _{2}$ fold changes of $c s n E^{c o m p}$ against the $\triangle l y S A$ background strain were included as control ( $\left.c s n E^{\text {comp } / \triangle} \Delta / y s A\right)$ and are close to 0 .

\begin{tabular}{|c|c|c|c|c|c|c|}
\hline $\begin{array}{c}\varnothing \log _{2} \mathrm{FC} \\
\Delta c s n E / \\
\Delta / y s A\end{array}$ & $\begin{array}{l}\text { SD } \\
\Delta c s n E / \\
\Delta l y s A \\
\end{array}$ & $\begin{array}{c}\varnothing \log _{2} \mathrm{FC} \\
\Delta c s n E / \\
c s n E^{c o m p}\end{array}$ & $\begin{array}{c}\text { SD } \\
\Delta c s n E / \\
\text { csnE } E^{\text {comp }}\end{array}$ & $\begin{array}{c}\varnothing \log _{2} \mathrm{FC} \\
c s n E^{c o m p} / \\
\Delta / y s A\end{array}$ & $\begin{array}{c}\text { SD } \\
\text { csnE }{ }^{\text {comp } /} \\
\text { DlysA }\end{array}$ & $\begin{array}{c}\text { Systematic } \\
\text { Name }\end{array}$ \\
\hline 2.90 & 1.13 & 2.74 & 1.11 & 0.21 & 0.12 & ANIA 05449 \\
\hline 1.47 & 0.83 & 1.48 & 0.99 & 0.04 & 0.17 & ANIA_01805 \\
\hline 1.42 & 0.76 & 1.50 & 0.93 & -0.04 & 0.14 & ANIA_06338 \\
\hline 1.31 & 1.87 & 1.47 & 1.95 & -0.30 & 0.05 & ANIA_07914 \\
\hline 1.28 & 0.55 & 1.36 & 0.74 & -0.10 & 0.25 & ANIA_00495 \\
\hline 1.23 & 0.58 & 1.23 & 0.59 & -0.07 & 0.07 & ANIA_01621 \\
\hline 1.16 & 0.65 & 1.24 & 0.74 & -0.09 & 0.09 & ANIA_04323 \\
\hline 1.13 & 0.57 & 1.09 & 0.40 & 0.03 & 0.15 & ANIA_10223 \\
\hline 1.10 & 0.29 & 0.93 & 0.34 & 0.37 & 0.11 & ANIA_02284 \\
\hline 1.05 & 0.15 & 1.01 & 0.30 & 0.08 & 0.07 & ANIA_01752 \\
\hline 0.97 & 0.22 & 0.69 & 0.42 & 0.24 & 0.24 & ANIA_04430 \\
\hline 0.97 & 0.51 & 1.20 & 0.81 & -0.15 & 0.20 & ANIA_00840 \\
\hline 0.96 & 0.22 & 0.97 & 0.31 & 0.06 & 0.07 & ANIA_07600 \\
\hline 0.95 & 0.65 & 0.75 & 0.77 & 0.07 & 0.20 & ANIA_03169 \\
\hline 0.92 & 0.29 & 0.81 & 0.26 & 0.10 & 0.09 & ANIA_08605 \\
\hline 0.92 & 0.45 & 1.11 & 0.55 & -0.05 & 0.16 & ANIA_05616 \\
\hline 0.90 & 0.38 & 0.79 & 0.53 & 0.13 & 0.08 & ANIA_02999 \\
\hline 0.86 & 0.46 & 0.84 & 0.67 & 0.08 & 0.16 & ANIA_03031 \\
\hline 0.85 & 0.23 & 0.57 & 0.27 & 0.14 & 0.04 & ANIA_00158 \\
\hline 0.82 & 0.43 & 0.81 & 0.82 & 0.03 & 0.37 & ANIA_07725 \\
\hline 0.80 & 0.40 & 0.98 & 0.74 & -0.10 & 0.27 & ANIA_10298 \\
\hline 0.80 & 0.09 & 0.88 & 0.17 & 0.00 & 0.05 & ANIA_05181 \\
\hline 0.75 & 0.71 & 1.18 & 0.71 & -0.34 & 0.04 & ANIA_07708 \\
\hline 0.72 & 0.15 & 0.78 & 0.22 & -0.01 & 0.11 & ANIA_02981 \\
\hline 0.72 & 0.33 & 0.75 & 0.58 & 0.11 & 0.12 & ANIA_01990 \\
\hline 0.71 & 0.25 & 0.83 & 0.32 & -0.04 & 0.03 & ANIA_05820 \\
\hline 0.70 & 0.35 & 0.93 & 0.56 & -0.16 & 0.23 & ANIA_04401 \\
\hline 0.69 & 0.11 & 0.77 & 0.01 & 0.04 & 0.05 & ANIA 07567 \\
\hline
\end{tabular}


Table S1: continued.

\begin{tabular}{|c|c|c|c|c|c|c|}
\hline $\begin{array}{c}\varnothing \log _{2} \mathrm{FC} \\
\Delta c s n E / \\
\Delta / y s A \\
\end{array}$ & $\begin{array}{c}\text { SD } \\
\Delta c s n E / \\
\Delta / y s A\end{array}$ & $\begin{array}{c}\varnothing \log _{2} \mathrm{FC} \\
\Delta c s n E / \\
c s n E^{c o m p}\end{array}$ & $\begin{array}{c}\text { SD } \\
\Delta c s n E / \\
\operatorname{csn} E^{c o m p}\end{array}$ & $\begin{array}{c}\varnothing \log _{2} \mathrm{FC} \\
c s n E^{\text {comp }} \\
\Delta / y s A \\
\end{array}$ & $\begin{array}{c}\text { SD } \\
\text { csnE } E^{\text {comp } /} \\
\Delta / y s A \\
\end{array}$ & $\begin{array}{c}\text { Systematic } \\
\text { Name }\end{array}$ \\
\hline 0.67 & 0.20 & 0.64 & 0.18 & 0.02 & 0.14 & ANIA 06655 \\
\hline 0.65 & 0.65 & 1.02 & 0.61 & -0.36 & 0.04 & ANIA 08277 \\
\hline 0.63 & 0.20 & 0.71 & 0.38 & -0.05 & 0.10 & ANIA_04462 \\
\hline 0.61 & 0.09 & 0.69 & 0.14 & -0.04 & 0.05 & ANIA_ 03712 \\
\hline 0.59 & 0.08 & 0.74 & 0.15 & -0.08 & 0.16 & ANIA_10219 \\
\hline 0.59 & 0.35 & 0.65 & 0.56 & -0.04 & 0.21 & ANIA_04793 \\
\hline 0.58 & 0.38 & 0.80 & 0.51 & -0.21 & 0.15 & ANIA_0893 \\
\hline 0.57 & 0.08 & 0.72 & 0.07 & -0.19 & 0.00 & ANIA_05904 \\
\hline 0.55 & 0.33 & 0.60 & 0.16 & -0.37 & 0.08 & ANIA_01662 \\
\hline 0.54 & 0.30 & 0.85 & 0.60 & -0.24 & 0.17 & ANIA_00354 \\
\hline 0.53 & 0.44 & 0.68 & 0.46 & -0.06 & 0.23 & ANIA_02976 \\
\hline 0.53 & 0.39 & 0.74 & 0.75 & -0.16 & 0.25 & ANIA 05999 \\
\hline
\end{tabular}

Table S2: Mean SILAC ratios of proteins, which abundances are increased in the presence of CsnE.

Logarithmized SILAC ratios (=fold changes (FC)) are shown in the table. Fold changes of $\triangle c s n E$ compared to the L-lysine auxotrophic background strain $\triangle l y s A$ are shown in the very left column $(\Delta c s n E / \Delta / y s)$ The FCs are the mean of three independent biological replicates. The standard deviation (SD) is shown as well. Furthermore, the mean fold changes of proteins in $\Delta c s n E$ compared to its complementation strain $c s n E^{\text {comp }}$ with the corresponding standard deviations are shown. $\log _{2}$ fold changes of $c s n E^{\text {comp }}$ against the $\triangle l y s A$ background strain were included as control ( $c s n E^{\text {comp } / \triangle l y s A)}$ and are close to 0 .

\begin{tabular}{|c|c|c|c|c|c|c|}
\hline $\begin{array}{c}\varnothing \log _{2} \mathrm{FC} \\
\Delta \operatorname{csn} E / \\
\Delta / y s A\end{array}$ & $\begin{array}{c}\text { SD } \\
\Delta c s n E / \\
\Delta l y s A\end{array}$ & $\begin{array}{c}\varnothing \log _{2} \mathrm{FC} \\
\Delta c \operatorname{csn} E / \\
c s n E^{\operatorname{comp}}\end{array}$ & $\begin{array}{c}\text { SD } \\
\Delta c s n E / \\
c s n E^{c o m p}\end{array}$ & $\begin{array}{c}\varnothing \log _{2} \mathrm{FC} \\
c s n E^{c o m p /} \\
\Delta / y s A\end{array}$ & $\begin{array}{c}\text { SD } \\
\text { csnE } E^{\text {comp } /} \\
\Delta / y s A\end{array}$ & $\begin{array}{c}\text { Systematic } \\
\text { Name }\end{array}$ \\
\hline-2.69 & 0.29 & -2.86 & 0.43 & -0.04 & 0.20 & ANIA_08547 \\
\hline-2.64 & 0.79 & -2.37 & 0.86 & -0.20 & 0.05 & ANIA_03344 \\
\hline-2.62 & 0.57 & -2.27 & 0.54 & -0.32 & 0.14 & ANIA 10296 \\
\hline-2.19 & 0.17 & -1.56 & 0.19 & -0.37 & 0.07 & ANIA_06058 \\
\hline-1.99 & 0.58 & -1.94 & 0.81 & -0.21 & 0.05 & ANIA_03524 \\
\hline-1.58 & 0.71 & -1.45 & 0.62 & -0.10 & 0.06 & ANIA_03674 \\
\hline-1.52 & 0.18 & -1.55 & 0.15 & 0.07 & 0.02 & ANIA_05823 \\
\hline-1.35 & 0.20 & -0.96 & 0.14 & -0.15 & 0.02 & ANIA_00858 \\
\hline-1.28 & 0.06 & -1.42 & 0.08 & 0.16 & 0.30 & ANIA_09180 \\
\hline-1.23 & 0.23 & -1.16 & 0.27 & -0.09 & 0.02 & ANIA_07062 \\
\hline-1.19 & 0.23 & -1.04 & 0.18 & -0.13 & 0.04 & ANIA_08009 \\
\hline-1.18 & 0.26 & -1.18 & 0.31 & 0.06 & 0.06 & ANIA_09148 \\
\hline
\end{tabular}


Table S2: continued.

\begin{tabular}{|c|c|c|c|c|c|c|}
\hline $\begin{array}{c}\varnothing \log _{2} \mathrm{FC} \\
\Delta c s n E / \\
\Delta / y s A \\
\end{array}$ & $\begin{array}{c}\text { SD } \\
\Delta c \operatorname{csn} E / \\
\Delta / y s A\end{array}$ & $\begin{array}{c}\varnothing \log _{2} \mathrm{FC} \\
\Delta c s n E / \\
c s n E^{c o m p}\end{array}$ & $\begin{array}{c}\text { SD } \\
\Delta c s n E / \\
\operatorname{csn} E^{c o m p}\end{array}$ & $\begin{array}{c}\varnothing \log _{2} \mathrm{FC} \\
c \text { csnE } \\
\Delta / y s A\end{array}$ & $\begin{array}{c}\text { SD } \\
\text { csnE }{ }^{c o m p} / \\
\Delta / y s A\end{array}$ & $\begin{array}{c}\text { Systematic } \\
\text { Name }\end{array}$ \\
\hline-1.18 & 1.53 & -0.86 & 1.27 & -0.40 & 0.07 & ANIA_08145 \\
\hline-1.17 & 0.34 & -0.91 & 0.43 & -0.27 & 0.06 & ANIA 04727 \\
\hline-1.13 & 0.33 & -0.62 & 0.35 & -0.14 & 0.30 & ANIA_03012 \\
\hline-1.11 & 0.91 & -1.01 & 0.90 & 0.00 & 0.04 & ANIA_04923 \\
\hline-1.04 & 0.49 & -0.83 & 0.34 & -0.10 & 0.14 & ANIA_03906 \\
\hline-1.04 & 0.17 & -0.62 & 0.14 & -0.35 & 0.05 & ANIA_03223 \\
\hline-1.03 & 0.53 & -0.87 & 0.69 & -0.16 & 0.05 & ANIA_01911 \\
\hline-1.02 & 0.52 & -0.63 & 0.50 & -0.33 & 0.04 & $\begin{array}{c}\text { ANIA_05082; } \\
\text { ANIA_10627; } \\
\text { tubB }\end{array}$ \\
\hline-1.01 & 0.38 & -0.97 & 0.11 & 0.00 & 0.02 & AN4908 \\
\hline-0.99 & 0.57 & -0.73 & 0.65 & -0.22 & 0.07 & ANIA_10709 \\
\hline-0.93 & 0.64 & -0.75 & 0.64 & -0.11 & 0.10 & ANIA_08182 \\
\hline-0.93 & 0.74 & -0.72 & 0.68 & -0.13 & 0.08 & ANIA_01394 \\
\hline-0.93 & 0.42 & -0.64 & 0.42 & -0.25 & 0.07 & ANIA_05586 \\
\hline-0.92 & 0.43 & -0.84 & 0.51 & -0.08 & 0.09 & ANIA_06004 \\
\hline-0.92 & 0.53 & -0.65 & 0.44 & -0.19 & 0.05 & ANIA_01158 \\
\hline-0.92 & 0.04 & -0.76 & 0.14 & -0.19 & 0.04 & ANIA_02532 \\
\hline-0.88 & 0.03 & -0.94 & 0.02 & -0.08 & 0.01 & ANIA_08815 \\
\hline-0.87 & 0.53 & -0.70 & 0.32 & -0.12 & 0.03 & ANIA_07262 \\
\hline-0.86 & 0.43 & -0.82 & 0.43 & -0.05 & 0.00 & ANIA_03112 \\
\hline-0.84 & 0.76 & -0.75 & 0.75 & -0.11 & 0.03 & ANIA_06688 \\
\hline-0.84 & 0.37 & -0.78 & 0.40 & -0.07 & 0.03 & ANIA_04042 \\
\hline-0.83 & 0.02 & -0.93 & 0.17 & 0.09 & 0.27 & ANIA_03829 \\
\hline-0.83 & 0.10 & -0.48 & 0.20 & -0.20 & 0.09 & ANIA_04591 \\
\hline-0.81 & 0.23 & -0.66 & 0.40 & -0.16 & 0.08 & ANIA_05895 \\
\hline-0.80 & 0.62 & -0.66 & 0.49 & -0.13 & 0.04 & ANIA_04463 \\
\hline-0.80 & 0.11 & -0.70 & 0.15 & -0.07 & 0.07 & ANIA_03636 \\
\hline-0.80 & 0.31 & -0.68 & 0.37 & -0.24 & 0.21 & ANIA_02925 \\
\hline-0.79 & 0.22 & -0.53 & 0.11 & -0.28 & 0.15 & ANIA_08233 \\
\hline-0.79 & 0.42 & -0.54 & 0.43 & -0.20 & 0.10 & ANIA 06341 \\
\hline-0.78 & 0.77 & -0.50 & 0.38 & -0.07 & 0.20 & ANIA_06266 \\
\hline-0.77 & 0.60 & -0.53 & 0.68 & -0.16 & 0.05 & ANIA_04667 \\
\hline-0.76 & 0.59 & -0.56 & 0.30 & -0.18 & 0.00 & ANIA_02756 \\
\hline-0.75 & 0.48 & -0.64 & 0.45 & -0.11 & 0.03 & ANIA_10614 \\
\hline-0.74 & 0.51 & -0.54 & 0.56 & -0.17 & 0.05 & ANIA_08012 \\
\hline-0.73 & 0.53 & -0.50 & 0.34 & -0.14 & 0.11 & ANIA_03026 \\
\hline-0.71 & 0.32 & -0.53 & 0.40 & -0.19 & 0.08 & ANIA_09094 \\
\hline
\end{tabular}


Table S2: continued.

\begin{tabular}{|c|c|c|c|c|c|c|}
\hline $\begin{array}{c}\varnothing \log _{2} \mathrm{FC} \\
\Delta c s n E / \\
\Delta / y s A \\
\end{array}$ & $\begin{array}{c}\text { SD } \\
\Delta c s n E / \\
\Delta / y s A\end{array}$ & $\begin{array}{c}\varnothing \log _{2} \mathrm{FC} \\
\Delta \operatorname{csn} E / \\
\operatorname{csn} E^{\operatorname{comp}}\end{array}$ & $\begin{array}{c}\text { SD } \\
\Delta c s n E / \\
c s n E^{c o m p} \\
\end{array}$ & $\begin{array}{c}\varnothing \log _{2} \mathrm{FC} \\
c s n E^{c o m p} / \\
\Delta / y s A\end{array}$ & $\begin{array}{c}\text { SD } \\
c s n E^{\text {comp }} / \\
\Delta / y s A\end{array}$ & $\begin{array}{c}\text { Systematic } \\
\text { Name }\end{array}$ \\
\hline-0.70 & 0.49 & -0.58 & 0.47 & -0.15 & 0.03 & ANIA_02867 \\
\hline-0.63 & 0.54 & -0.63 & 0.47 & 0.03 & 0.06 & ANIA_07146 \\
\hline-0.61 & 0.04 & -0.76 & 0.26 & 0.20 & 0.25 & ANIA_01810 \\
\hline-0.59 & 0.14 & -0.67 & 0.38 & 0.10 & 0.01 & ANIA_00327 \\
\hline-0.59 & 0.53 & -0.60 & 0.60 & 0.04 & 0.07 & ANIA_01971 \\
\hline-0.58 & 0.64 & -0.52 & 0.52 & -0.08 & 0.06 & ANIA_02068 \\
\hline-0.56 & 0.64 & -0.81 & 0.76 & 0.34 & 0.10 & ANIA_04197 \\
\hline-0.55 & 0.49 & -0.63 & 0.37 & 0.07 & 0.12 & ANIA_02436 \\
\hline-0.54 & 0.42 & -0.67 & 0.55 & 0.08 & 0.07 & ANIA_07011 \\
\hline-0.54 & 0.19 & -0.70 & 0.14 & 0.09 & 0.03 & ANIA_07208 \\
\hline-0.50 & 0.52 & -0.55 & 0.38 & 0.07 & 0.14 & ANIA_02435 \\
\hline
\end{tabular}




\section{List of Abbreviations}

\begin{tabular}{|c|c|}
\hline 3D & Three-dimensional \\
\hline$\%$ & Percent \\
\hline${ }^{\circ} \mathrm{C}$ & Degree Celsius \\
\hline$\alpha$ & Alpha \\
\hline$\beta$ & Beta \\
\hline$\Delta$ & Deletion \\
\hline$\mu g$ & Microgram \\
\hline$\mu l$ & Microliter \\
\hline$\mu \mathrm{M}$ & Micromolar \\
\hline aa & Amino acid(s) \\
\hline & Alanine alanine \\
\hline$A D$ & Activation domain \\
\hline ADP & Adenosine diphosphate \\
\hline AspGD & Aspergillus genome database \\
\hline asex & Asexual \\
\hline ATP & Adenosine triphosphate \\
\hline $\mathrm{BiFC}$ & Bimolecular fluorescence complementation \\
\hline BLAST & Basic local alignment search tool \\
\hline $\mathrm{bp}$ & Base pairs \\
\hline btm & Bottom \\
\hline C & Cysteine \\
\hline CCR & Carbon catabolite repression \\
\hline $\mathrm{C}_{2} \mathrm{H}_{2}$ & $\mathrm{Cys}_{2} \mathrm{His}_{2}$ zinc finger DNA binding domain \\
\hline CADRE & Central Aspergillus data repository \\
\hline cDNA & Complementary DNA \\
\hline CID & Collision-induced dissociation \\
\hline $\mathrm{cm}$ & Centimeter \\
\hline comp & complementation \\
\hline COP9 & Constitutive photomorphogenesis complex 9 \\
\hline CRL & Cullin-RING ligase \\
\hline CSN & COP9 signalosome \\
\hline C-terminus & Carboxy terminus \\
\hline Cul & Cullin \\
\hline$d$ & $\operatorname{Day}(\mathrm{s})$ \\
\hline D & Aspartic acid \\
\hline DBD & DNA binding domain \\
\hline Dba & Derivative of benzaldehyde \\
\hline DenA & Deneddylase A \\
\hline DIC & Differential intereference contrast \\
\hline DNA & Deoxyribonucleic acid \\
\hline DTT & Dithiothreitol \\
\hline
\end{tabular}




\begin{tabular}{|c|c|}
\hline DUB & Deubiquitinating enzymes \\
\hline DUSP & Domain present in ubiquitin-specific proteases \\
\hline EDTA & Ethylenediaminetetraacetic acid \\
\hline e.g. & Exempli gratia $=$ for example \\
\hline elF3 & Eukaryotic initiation factor 3 \\
\hline ERAD & Endoplasmatic reticulum-associated protein degradation \\
\hline EV & Empty vector \\
\hline FungiDB & Fungi database \\
\hline g & Gram \\
\hline gDNA & Genomic DNA \\
\hline GFP & Green fluorescent protein \\
\hline GOI & Gene of interest \\
\hline GTP & Guanosin triphosphate \\
\hline h & Hour(s) \\
\hline $\mathrm{H}$ & histidine \\
\hline $\mathrm{H} 2 \mathrm{~A}$ & Histone \\
\hline $\mathrm{H}_{2} \mathrm{O}_{2}$ & Hydrogen peroxide \\
\hline HECT & Homologous to E6 associated protein C-terminus \\
\hline HPLC & High performance liquid chromatography \\
\hline ir & Inner region \\
\hline JAMM & JAB1/MPN/Mov34 metalloenzyme \\
\hline K & Lysine \\
\hline $\mathrm{kb}$ & Kilo bases \\
\hline $\mathrm{kDa}$ & Kilo Dalton \\
\hline I & Liter \\
\hline LB & Lysogeny broth \\
\hline LC & Liquid chromatography \\
\hline LFQ & Label free quantification \\
\hline LUBAC & Linear ubiquitin chain assembly complex \\
\hline Lys4 & Medium, D4 labeled L-lysine $* 2 \mathrm{HCl}$ \\
\hline Lys8 & Heavy, ${ }^{13} \mathrm{C}{ }^{15} \mathrm{~N}$ L-lysine $* \mathrm{HCl}$ \\
\hline M & Molar (mol/l) \\
\hline MAP & Mitogen-activated (kinase) \\
\hline $\mathrm{mg}$ & Milligram \\
\hline $\min$ & Minute(s) \\
\hline MINDY & Motif interacting with Ub-containing novel DUB family \\
\hline MJD & Machado-Josephin domain containing proteases \\
\hline $\mathrm{ml}$ & Milliliter \\
\hline MM & Minimal medium \\
\hline $\mathrm{mM}$ & Millimolar \\
\hline $\mathrm{mm}$ & Millimeter \\
\hline MPN & Mpr1 and Pad1 N-terminal domain \\
\hline
\end{tabular}




\begin{tabular}{|c|c|}
\hline $\mathrm{mr}$ & middle region \\
\hline mRNA & Messenger RNA \\
\hline MS/MS & Tandem mass spectrometry \\
\hline natRM & Nourseothricin recyclable resistance marker \\
\hline Nedd8 & $\begin{array}{l}\text { Neuronal precursor cell expressed, developmentally } \\
\text { downregulated } 8\end{array}$ \\
\hline N8 & Nedd8 \\
\hline NES & Nuclear export signal \\
\hline $\mathrm{NF}-\mathrm{Kb}$ & $\begin{array}{l}\text { Mammalian nuclear factor kappa-light-chain-enhancer of } \\
\text { activated B-cells }\end{array}$ \\
\hline NLS & Nuclear localization sequence \\
\hline N-terminal & Amino-terminal \\
\hline $\mathrm{o} / \mathrm{n}$ & Over night \\
\hline OE & Overexpression \\
\hline or & Outer region \\
\hline ORF & Open reading frame \\
\hline OTU & Ovarian tumor proteases \\
\hline$P$ & Promoter \\
\hline p.c. & Personal communication \\
\hline $\mathrm{PCl}$ & Proteasome, COP9, eukaryotic initiation factor 3) \\
\hline PCR & Polymerase chain reaction \\
\hline PEST & Proline, glutamic acid, serine and threonine rich region \\
\hline phleoRM & Phleomycin recyclable resistance marker \\
\hline PMG & Photomicrograph \\
\hline PTM & Posttranslational modification \\
\hline PKS & Polyketide synthase \\
\hline pyroA & Pyridoxine biosynthesis protein \\
\hline qRT-PCR & Quantitative real-time PCR \\
\hline $\mathrm{R}$ & Resistance \\
\hline $\mathrm{R}$ & Arginine \\
\hline $\operatorname{Rbx}$ & RING box domain containing protein \\
\hline RFP & Red fluorescent protein \\
\hline RING & Really interesting new gene \\
\hline RNA & Ribonucleic acid \\
\hline rRNA & Ribosomal RNA \\
\hline rpm & Rounds per minute \\
\hline rt & Room temperature \\
\hline S & Serine \\
\hline SAGA & Spt-Ada-Gcn5-acetyltransferase \\
\hline SCF & Skp1-Cul1-Fbox \\
\hline SDS & Sodium dodecyl sulfate \\
\hline SEM & Standard error of the mean \\
\hline sex & Sexual \\
\hline
\end{tabular}


SILAC Stable isotope labelling with amino acids in cell culture

Skp1

$\mathrm{SM}(\mathrm{s})$

spp.

SUMO

STDEV

$\mathrm{T}$

TAP

TGF- $\beta$

TNF

$\mathrm{Ub}$

$\mathrm{UCH}$

UPS

USP

UTR

veg

$\mathrm{v} / \mathrm{v}$

$\mathrm{w} / \mathrm{v}$

Wnt

WT

$\mathrm{Y} 2 \mathrm{H}$

YPED

YFP

$\mathrm{Zn}$
S-phase kinase-associated protein 1

Secondary metabolite(s)

Species

Small ubiquitin-like modifier

Standard deviation

Terminator

Tandem affinity purification

Transforming growth factor beta

Tumor necrosis factor

Ubiquitin

Ubiquitin C-terminal hydrolases

Ubiquitin-proteasome pathway

Ubiquitin-specific protease

Untranslated region

Vegetative

Volume per volume

Weight per volume

Wingless-type

Wild type

Yeast-two-hybrid

Yeast peptone-extract-dextrose growth medium

Yellow fluorescent protein

Zinc 


\section{List of Tables}

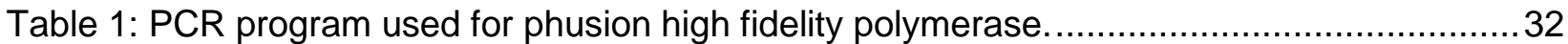

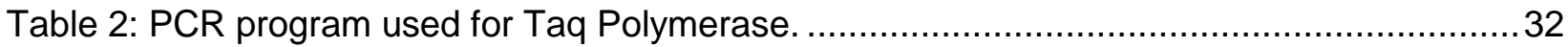

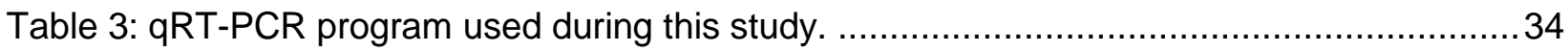

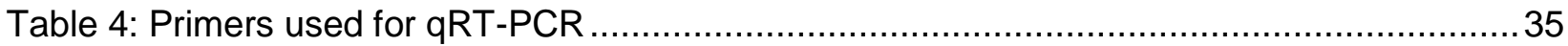

Table 5: Primers used in this study for amplification, sequencing and cloning of plasmids. .......38

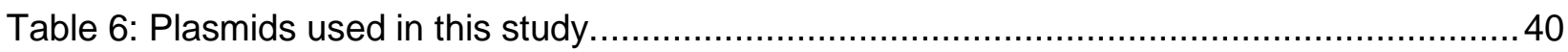

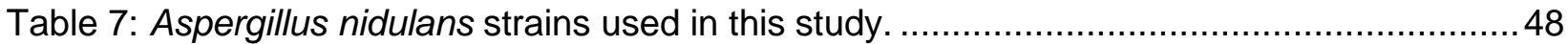

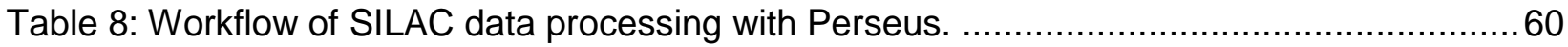

Table 9: Workflow for GFP pull down analyses with the Perseus Software ...............................62

Table 10: Labeling efficiency with SILAC amino acids.......................................................6

Table 11: Proteins with increased abundance during vegetative growth of $A$. nidulans in $\Delta c s n E$.

Table 12: Proteins with decreased abundance during vegetative growth in a $\triangle c s n E$ strain. .......77

Table 13: Putative orthologs of human Usp15 in A. nidulans and putative orthologous proteins in

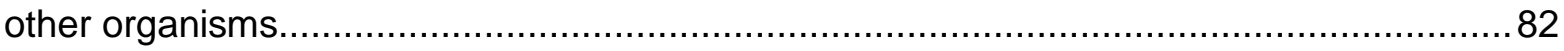

Table 14: Functional groups of proteins identified with LC/MS-MS in pull downs of UspA-GFP and UspA ${ }^{\mathrm{AA}}$-GFP.

Table 15: Proteins that were identified only in the pull down of UspA ${ }^{\text {AA }}$-GFP, but not in the pull

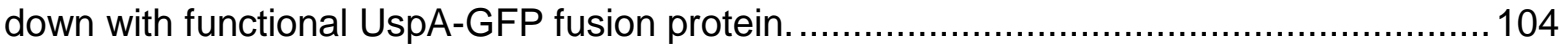

Table 16: Proteins exclusively identified in UspA-GFP pull down. ........................................105

Table 17: An overview about deubiquitinating enzymes in A. nidulans. ................................125

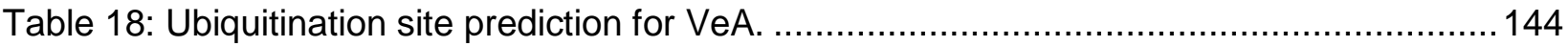

Table S1: Mean SILAC ratios of proteins, which abundances are decreased in the presence of CsnE.

Table S2: Mean SILAC ratios of proteins, which abundances are increased in the presence of CsnE. 


\section{List of Figures}

Figure 1: Ubiquitin is highly conserved between eukaryotes............................................. 3

Figure 2: Localization of lysine residues in the three dimensional structure of ubiquitin. .............4

Figure 3: Transfer of ubiquitin molecules to target proteins............................................ 6

Figure 4: Schematic representation of the modular architecture of eukaryotic SCF E3 ligases....9

Figure 5: Schematic representation of the COP9 signalosome architecture. .......................... 10

Figure 6: Diverse functions of deubiquitinating enzymes in the ubiquitin cycle. ......................14

Figure 7: Life cycle of Aspergillus nidulans. ................................................................... 19

Figure 8: Localization and interaction partners of $\mathrm{VeA}$, the master regulator of development and

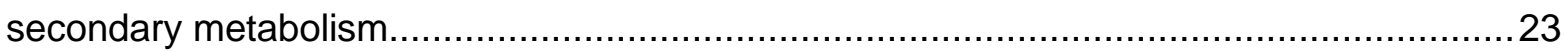

Figure 9: Schematic representation of the recycable marker system. ....................................37

Figure 10: Schematic representation of the L-lysine and L-arginine biosyntheses in $A$. nidulans.

Figure 11: Phenotypes of $A$. nidulans L-lysine and L-arginine auxotrophic strains.

Figure 12: Schematic representation of the workflow for the SILAC amino acid incorporation test.

Figure 13: Incorporation efficiency of isotopically-labeled L-lysine variants into fungal proteins during vegetative growth.

Figure 14: CsnE is required for the development of asexual spores, cleistothecia and regulation

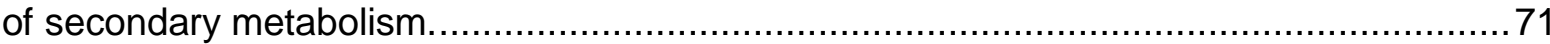

Figure 15: Scatter plot of proteins identified in SILAC experiment. ........................................ 73

Figure 16: Classification of proteins, which abundance is decreased by CsnE. .....................76

Figure 17: Classification of proteins, which abundance is increased by CsnE. .......................80

Figure 18: Multiple sequence alignments of fungal USPs. ................................................. 83

Figure 19: AN6354 encodes UspA and is a member of the fungal USP family. ......................84

Figure 20: $A$. nidulans UspA interacts with COP9 signalosome subunits in yeast-two-hybrid assay.

Figure 21: CsnE causes repressed expression of genes encoding for putative USPs during

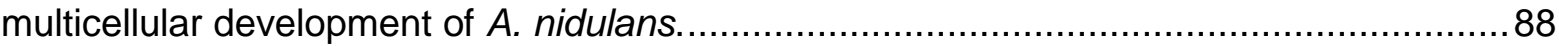

Figure 22: csnE transcript levels are independent of UspA........................................... 89

Figure 23: CsnE protein levels are independent of the deubiquitinase UspA. ........................90

Figure 24: UspA interacts with CsnB and CsnF in bimolecular fluorescence complementation

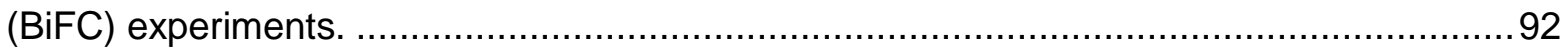

Figure 25: Functional and non-functional UspA-GFP proteins accumulate close to nuclei........94

Figure 26: Subpopulations of UspA-GFP and UspA AA -GFP co-localize with RFP-CsnB. ..........95

Figure 27: The fusion protein UspA ${ }^{\mathrm{AA}}$-GFP is transcribed and expressed like UspA-GFP........97

Figure 28: UspA deubiquitinates proteins during fungal growth and development. ..................98

Figure 29: UspA-GFP and UspA ${ }^{A A}$-GFP were enriched in GFP pull down experiments............99

Figure 30: Identification of putative interaction partners of UspA-GFP and UspA ${ }^{\text {AA }}$-GFP with

LC/MS-MS.

Figure 31: AN7422 transcript levels are upregulated in the absence of functional COP9

signalosome similar to uspA

Figure 32: UspA induces conidiospore formation in A. nidulans. 
Figure 33: UspA is required for conidiospore formation.

Figure 34: The lack of pyridoxine supplementation emphasizes the uspA deletion phenotype.108

Figure 35: UspA is required for the early steps of cleistothecia development.

Figure 36: UspA is important for correct timing in early sexual development.

Figure 37: The lack of pyridoxine in the culture medium enhances the deletion phenotype. .... 112

Figure 38: UspA causes repressed expression of genes of the derivative of benzaldehyde (dba) cluster during multicellular development.

Figure 39: UspA destabilizes VeA-GFP protein abundance during late developmental time

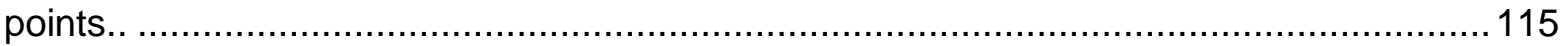

Figure 40: VelB-GFP protein abundance is independent of UspA.

Figure 41: VeA-GFP and VelB-GFP localize inside nuclei independently of the deubiquitinase UspA.

Figure 42: VeA pulls VelB and Dba cluster proteins in $\Delta u s p A^{p y r o A}$ in GFP pull down experiments.

Figure 43: Deubiquitinating enzymes can be divided into six subfamilies.

Figure 44: The COP9 signalosome influences uspA gene transcription.

Figure 45: Cartoon representation of the human COP9 signalosome helical bundle.

Figure 46: UspA might interact with the COP9 signalosome during the catalysis of deubiquitinating reactions.

Figure 47: UspA accumulates primarily close to nuclei and appears in smaller subpopulations

inside the nucleus as well as in the cytoplasm.

Figure 48: Putative modes of regulation of $\mathrm{VeA}$ through the deubiquitinase UspA.

Figure 49: Fungal velvet domain proteins show high similarities to the mammalian NF-KB RHD domain containing transcription factor family.

Figure 50: Model of the interplay between the COP9 signalosome deneddylase and the UspA deubiquitinase. 


\section{Acknowledgements}

First of all I would like to thank Prof. Gerhard H. Braus for his constant support during the time of my PhD. I joined his reseach group for my master thesis and continued for my PhD. I am thankful for the great supervision, fruitful disccussions and the time he invested in me and my work. I am grateful for the opportunity to attend many national and international conferences during the time of my PhD.

Secondly, I want to thank my Thesis Committee Members Dr. Achim Dickmanns and Prof. Dr. Kai Tittmann for helpful advices and suggestions during the regular meetings. Additionally, thanks goes to Prof. Dr. Stefanie Pöggeler, Jun.-Prof. Dr. Kai Heimel Prof. and Prof. Dr. Heike Krebber for being members of my Examination board.

I am grateful for the opportunity to be a part of the Göttingen Graduate School for Neuroscience, Biophysics and Molecular Biosciences (GGNB) doctoral programme "Biomolecules: StructureFunctions-Dynamics". This offered great opportunities to attend courses to further deepen the understanding in different research fields and coming into contact with other PhD students. The cooperativeness of members of the GGNB office was outstanding in all situations I had to contact them during the time of my PhD. I am thankful for their financial support, which enabled the attendance at many scientific conferences. Furthermore, I am very grateful to got the chance of being a member of the SFB860, which enabled the work on my PhD project and supplied constant financial support. The annual meetings of the SFB860 were a convient possibility to come into contact with other researchers and to increase the scientific network.

Deep thanks goes to Dr. Oliver Valerius and Dr. Kerstin Schmitt for doing LC/MS-MS measurements for my work, discussing results, proofreading parts of my thesis and all the sciencitif and non-scientific conversations during the time of my PhD. I am really grateful for their constant support especially, but not only, for the SILAC project.

Without the former and current members of the Lab1.134 my PhD thesis would not have been possible. A deep thanks deserves Gaby for her constant technical and mental support. I enjoyed all the work related and private conversations. In all situations I could count on her help and support. Furthermore, I have to thank Anna. We shared the lab already during our master thesis. During all the time it was a great pleasure to work with you, we shared good and bad moments 
in the lab, and finally we are finishing together. I cannot wait to celebrate with you! Warm thanks goes to Fruzsi for all the fruitful discussions, advices and fun we had in the lab. I would like to thank Mirit for all the conversations, discussion and fun we had during the last years. Last but not least thanks goes to former lab members Josh and Rebekka for all the things I learned from them. It was a pleasure to share a lab with all of you! A special thanks goes to Rebekka for proofreading my thesis and be always available for all kind of questions during the whole time.

I would like to thank all members of the Department of Molecular Microbiology and Genetics for the great working atmosphere and the constant willingness to help during all the time of my $\mathrm{PhD}$. Thanks goes to Dr. Blagovesta Popova for introducing me into work with S. cerevisiae. A special thanks goes to our lunch group Anna, Fruzsi, Anja, Karl and Bine for all the great moments we had in- and outside of the lab. I am deeply grateful to be able to call you my friends. Thanks for the constant support, helpful discussion and advices. Thank you all for proofreading my thesis. Thereby, a sincere thanks goes to Karl spending really a lot of time to proofread my thesis and my manuscript.

Last but not least, I would like to express my deepest thanks to my parents for their constant encouragement and support. Finally, my heartfelt gratitude goes to my boyfriend Kristian. Without his constant support and love this all would not have been possible! 